\title{
L'obligation morale et l'ethique de la prosperite : le retour du sujet responsable
}

Citation for published version (APA):

Fragniere, G. L. (1993). L'obligation morale et l'ethique de la prosperite : le retour du sujet responsable.

[Doctoral Thesis, Maastricht University]. Rijksuniversiteit Limburg. https://doi.org/10.26481/dis.19930623gf

Document status and date:

Published: 01/01/1993

DOI:

10.26481/dis.19930623gf

Document Version:

Publisher's PDF, also known as Version of record

\section{Please check the document version of this publication:}

- A submitted manuscript is the version of the article upon submission and before peer-review. There can be important differences between the submitted version and the official published version of record.

People interested in the research are advised to contact the author for the final version of the publication, or visit the DOI to the publisher's website.

- The final author version and the galley proof are versions of the publication after peer review.

- The final published version features the final layout of the paper including the volume, issue and page numbers.

Link to publication

\footnotetext{
General rights rights.

- You may freely distribute the URL identifying the publication in the public portal. please follow below link for the End User Agreement:

www.umlib.nl/taverne-license

Take down policy

If you believe that this document breaches copyright please contact us at:

repository@maastrichtuniversity.nl

providing details and we will investigate your claim.
}

Copyright and moral rights for the publications made accessible in the public portal are retained by the authors and/or other copyright owners and it is a condition of accessing publications that users recognise and abide by the legal requirements associated with these

- Users may download and print one copy of any publication from the public portal for the purpose of private study or research.

- You may not further distribute the material or use it for any profit-making activity or commercial gain

If the publication is distributed under the terms of Article $25 \mathrm{fa}$ of the Dutch Copyright Act, indicated by the "Taverne" license above, 


\section{L'obligation morale}

et

\section{l'éthique de la prospérité}

Le retour du sujet responsable 
Une édition commerciale de ce livre est disponible auprès des Presses Interuniversitaires européennes (Bruxelles)

sous le

ISBN 90 - $5201-304-7$

D / $1993 / 5678 / 05$ 


\section{L'obligation morale}

\section{et \\ l'éthique de la prospérité}

Le retour du sujet responsable

\section{PROEFSCHRIFT}

ter verkrijging van de graad van doctor aan de Rijksuniversiteit Limburg te Maastricht, op gezag van de Rector Magnificus, Prof. mr. M. J. Cohen, volgens het besluit van het College van Dekanen,

in het openbaar te verdedigen op woensdag, 23 juni 1993 om 16.00 uur

door

Gabriel Léon Fragnière 


\section{Promotores:}

Prof.dr. E.J.J.M. Kimman, RL

Prof.dr. W. Albeda, RL

\section{Beoordelingscommissie:}

Prof.dr. J.G. Backhaus, RL (voorzitter)

Prof.dr. Ph. Van Parijs, Université Catholique de Louvain, Louvain la Neuve

Prof.dr. J.M.M. de Valk, Erasmus Universiteit Rotterdam

Prof.dr.ir. G.H. de Vries, RL 


\section{Table des matières}

INTRODUCTION: Les Thèses, Histoire d'un manuscrit

Chapitre 1:

La question: Le retour de la philosophie morale

La pensée économique, le bien, le mal et l'éthique

de la prospérité

Une question en situation historique

Crise de l'économie ou crise de la théorie économioque

Prédominance du libéralisme et triomphe de l'économie

de marché

La faillite du système communiste

Le désarroi de la pensée contemporaine

Besoin d'une éthique universelle

Rétablir la philosophie dans le moralisme économique

Chapitre 2:

Historique de l'obligation morale

Première section:

Le développement de la conscience et la révélation du devoir

1. L'obligation socratique et la morale antique …………...................36

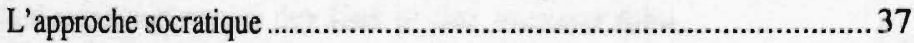

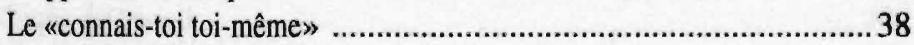

La finalité dans la morale antique ….................................................. 41

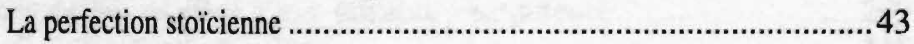

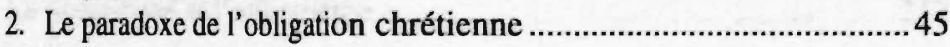


Conscience et rationalisation ......................................................51

L'obligation morale ...................................................................53

Obligation et morale d'amour …………....................................54

3. L'absolu du devoir kantien ...............................................................5

L'a priori et le formalisme ......................................................57

La moralité par le devoir ............................................................5 59

Le sujet du devoir et la conscience morale .................................61

Deuxième section:

L'explication de l'obligation et la dissolution du devoir ..................64

4. Une explication psychologique (Georges Fulliquet) ...................66

5. Une explication vitaliste (Jean-Marie Guyau) .............................69

6. Une explication sociologique (Emile Durkheim) ........................73

7. L'explication bergsonienne (Henri Bergson) ……………............79

Troisième Section:

L'obligation aujourd'hui ............................................................... 86

Chapitre 3: Analyse de la morale et connaissance de soi -

Les deux dimensions de la morale (analyse conceptuelle).....92

Le champ de la recherche morale ................................................93

Ambiguïté de l'anthropologie morale .................................................95

Les concepts de la philosophie morale .............................................97

La double dimension de la morale .................................................. 100

L'adjectif «moral» et ses formes négatives ........................................ 101

1 - MORAL - A-MORAL: Le fait moral et ses implications ..... 104

Signification humaine de la subjectivité morale ...............................106

La révélation de la liberté ...........................................................112

Intention et finalité .................................................................115

La responsabilité ......................................................................... 117

Le fait de la morale ................................................................. 120

2 - MORAL - IM-MORAL: La valeur morale

et l'objectivité inter-subjective ...................................................122

Le jugement de valeur et la norme ............................................... 124

L'objectivité morale ...................................................................... 127 
Chapitre 4:

L'obligation morale vécue (analyse existentielle)

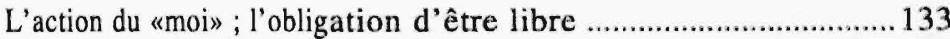

Les temps de l'action : l'obligation de créer la moralité ...................136

Les causes de l'action : l'obligation de se faire soi-même ...............138

Le conflit moral : l'obligation de se dépasser ......................................141

L'appel d'autrui : l'obligation réelle ......................................................... 144

La personne comme devoir-être .........................................................146

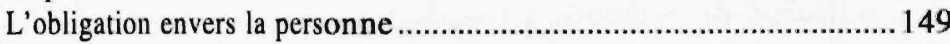

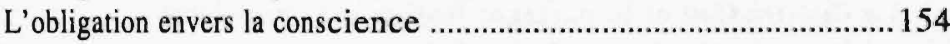

Chapitre 5:

Les illusions de l'obligation, ou la société-objet...................157

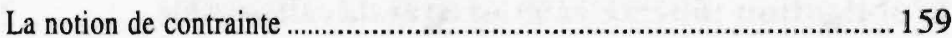

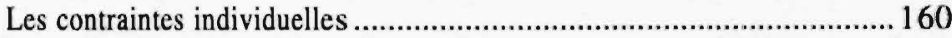

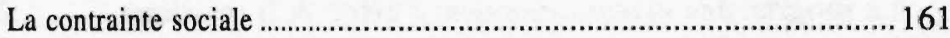

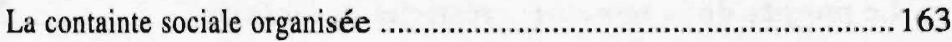

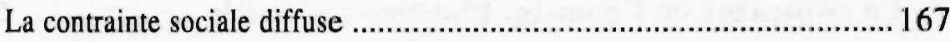

Le dépassement de la contrainte morale ............................................171

Obligation formelle et obligation matérielle ........................................173

Chapitre 6:

L'obligation envers autrui, ou la société-valeur .................. 175

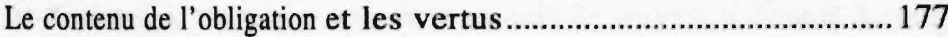

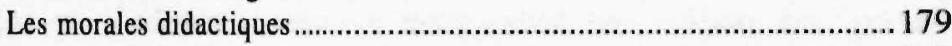

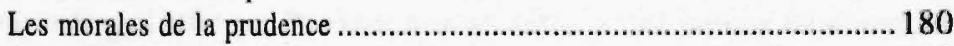

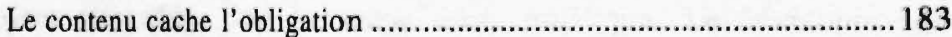

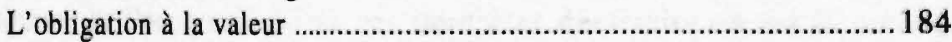

L'obligation pour autrui et l'obligation sociale .................................. 190

Chapitre 7:

Le retour du sujet responsable

L'éthique de la prospérité: paradigme et contrainte ............................ 198

Limites de l'éthique de la prospérité ...................................................203

L'équivoque permutation des fins et des moyens dans

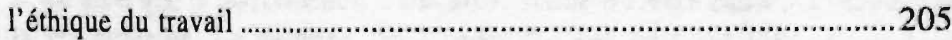

Renversement des valeurs de l'action humaine ................................207

L'homme pris au piège d'une éthique immanente ............................209

Le libéralisme et l'utilitarisme .........................................................212 
Le capitalisme, le néo-libéralisme et l'éthique sociale 217

Le sujet responsable

Chapitre 8 :

La construction de l'histoire et la justice 225

Rupture symbolique et conscience de l'humanité .............................227

Sociétés et sujets dans la construction de l'histoire .........................231

La Justice: de la théorie à la valeur ...............................................235

La distribution et le partage: justice et responsabilité ......................238

L'Etat:sujet du devoir de justice ....................................................242

Vers une éthique de la justice …........................................................247

Chapitre 9 :

L'obligation morale et le progrès de l'humanité ..................252

Le progrès des valeurs morales: l'effort de la conscience................254

Le progrès de la moralité: créativité de l'individu ............................261

La croissance de l'humain: l'homme responsable ............................264

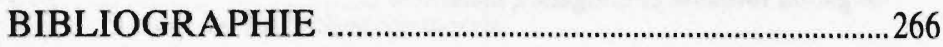

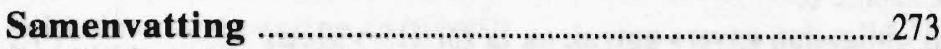

Curriculum Vitae ..................................................................... 


\section{Les Thèses Histoire d'un manuscrit}

Ce livre est une recherche philosophique sur l'obligation morale. Il propose une perspective nouvelle sur les questions éthiques que soulève la vie économique et sociale de notre époque. Le premier but de cette tentative est de situer le sujet, l'homme individuel, la personne, au centre de toute réflexion sur le destin de l'humanité.

Nous partons d'une constatation, à savoir que la vie en société, et plus particulièrement la vie économique et le monde du travail, a évolué de telle façon que l'individu se retrouve marginalisé par rapport aux grandes évolutions qui l'affectent. Pourtant nos sociétés n'ont jamais eu autant de moyens disponibles pour faire une autre politique. Le développement fulgurant de l'économie durant ces dernières décennies en est la preuve. Mais les instruments intellectuels n'ont pas progressé de la même façon. La pensée actuelle sur l'homme - anthropologie, sociologie, psychanalyse, science politique, et de façon générale de toutes les sciences humaines semble avoir accepté de se soumettre au pouvoir des faits dominants, avec pour conséquence une tendance à objectiver toute connaissance de l'être humain. Dès lors la conscience de soi se dilue progressivement dans un immense processus intellectuel anonyme justifant avant tout le fonctionnement du groupe social et l'emprise de ce demier sur l'action de ses membres.

C'est ainsi que la performance s'est substituée à l'être dans l'évaluation de la vie humaine. La destinée de l'individu en tant qu'être unique et irremplaçable a inconsciemment glissé de l'affirmation de son existence aux fonctions et rôles qu'il remplit dans son milieu. La valeur de l'homme 
n'est plus dans ce qu'il est, mais dans ses performances et son travail. Pour comprendre et maîtriser son destin dans cet environnement, l'homme est forcé de sortir de lui-même, d'abandonner toute introspection et toute intuition réflexive de son être pour concentrer son intelligence et sa volonté sur les raisons de vivre proposées par le conditionnement dont il est l'objet. II fait objectivement ce qu'il faut, mais il a perdu le sens intérieur de sa vocation.

C'est ainsi que l'action socialement déterminée a peu à peu pris la place de la conscience de soi. Une nouvelle formule se propose pour vaincre le doute: j'agis, donc je suis. L'activisme a remplacé la conscience. Les morales empiriques répondent à une nouvelle logique qui explique avant tout le comment des actions humaines plutôt que leur sens et leur pourquoi. Ce ne sont pas les personnes, mais leurs fonctions dans les structures économiques dominantes, en particulier les entreprises, qui sont devenues l'objet d'élection des sciences sociales. Mais en réduisant l'homme à sa seule dimension utilitaire, la pensée le détache d'autres dimensions de l'être, en particulier du lien qu'il entretenait avec l'image transcendante d'un Dieu qui, en l'enracinant dans l'absolu, le protégeait contre les abus du monde. Le Bien et le Mal ne se rattachent plus à l'immuable. Aux yeux de la société performante, ce relativisme ouvre la voie à tous les possibles. Tout est devenu libre, car il n'y a plus d'autre norme que le succès.

Mais cette liberté, aussi envahissante qu'elle apparaît illimitée, peut être un piège. Elle a en effet des implications dont l'individu n'est pas toujours conscient. Privé de points de repère qui s'accrochaient à des valeurs le dépassant, l'homme doit inventer, choisir, créer ses propres fins, et devenir ainsi responsable de tout; de sa vie propre d'abord, de la société dans laquelle il vit et qu'il doit faire fonctionner, des valeurs au nom desquelles il juge et est lui-même jugé et, pour finir, de l'humanité dont il contribue à construire le destin.

A cette liberté sans limites corrrespond une responsabilité de même ampleur. Sur quoi se fonde-telle? Quelle en est la nature et le rôle dans la constitution de la condition humaine? Quelle place donne-t-elle à l'homme dans la vie sociale? En quels termes peut-on en parler? Comment peut-on la comprendre intellectuellement? C'est dans la perspective que soulèvent de telles questions et pour tenter d'y répondre que nous nous proposons de retrouver le sens de l'obligation morale.

Le but de ce travail est donc de redonner à l'obligation morale le rôle qu'elle peut et doit remplir pour justifier et soutenir la responsabilité humaine dans les conceptions éthiques dont notre société a besoin en cette fin de siècle. 
La richesse est devenue non seulement possible pour un grand nombre, mais réelle. Elle résulte du fonctionnement plus efficace et plus libre de l'économie. Même si elle n'est pas encore équitablement distribuée dans le monde, y compris dans les pays riches, cette richesse et sa production sont à l'origine d'un nouveau système de valeurs, de comportements et d'une attitude morale qui constituent ensemble ce que nous appelons l'éthique de la prospérité. Elle consiste à faire de l'accroissement de la richesse la fin dernière de toutes les actions humaines. Cette éthique met l'individu au service de l'économie, et implique une vision exclusivement fonctionnelle de l'homme dans laquelle, à la limite, celui-ci perd sa liberté. La théorie économique se propose dès lors elle-même comme une éthique et détermine ainsi les normes et la finalité de l'action. Elle fait de tous les agents économiques, individus et collectivités, des objets au service du maintien et de la croissance du bien-être matériel.

Pour ne pas perdre son âme devant l'omniprésence de la prospérité, l'homme doits'affirmer comme sujet. Il doit recouvrer sa vraie responsabilité dans le choix de ses propres fins. La philosophie doit revendiquer son rôle dans cette renaissance et intervenir dès lors dans le débat sur les finalités de l'économie. Nous voyons dans la notion d'obligation morale, - que nous reconstruisons à partir de ses développements dans l'histoire de la philosophie occidentale - une des ressources essentielles de la pensée pour construire une nouvelle anthropologie, c'est-à-dire une connaissance de l'homme par lui-même qui puisse redonner au sujet la conscience de son être individuel et social, et le sens de sa liberté.

Une telle attitude morale, libre et responsable, fondée sur le sens de l'obligation, doit être celle de tous les agents économiques. Il existe donc une forme d'obligation morale, une responsabilité publique spécifique qui s'adresse aux personnes morales, qui concerne les collectivités et les Etats, et qui se propose comme telle à ceux qui en ont la charge: chefs d'entreprises, politiques, fonctionnaires et hommes d'Etat. L'idéologie libérale, toute puissante en cette fin de siècle, animée par un capitalisme qui n'a plus à lutter contre l'ennemi historique qu'était le communisme, entretient à travers le slogan du «moins d'Etat» l'illusion d'une vie sociale satisfaisante sans société responsable, en s'appuyant sur une valorisation fallacieuse de l'éthique de la prospérité. Le sens de l'obligation peut permettre de retrouver une relation harmonieuse entre individu et société, entre citoyens libres et Etat responsable. La société elle-même peut devenir une «société-sujet». 
C'est dans l'idée de «Justice» que s'exprime la notion d'obligation morale pour les collectivités et les Etats. Mais à l'instar des valeurs qui se découvrent dans chacun des actes de l'individu, la Justice n'est pas intemporelle; elle doit être construite et renouvelée en permanence. Elle a donc une histoire, à l'instar de la liberté et de la démocratie. C'est pourquoi ce n'est pas la théorie de la justice qui peut nous sortir de l'impasse éthique dans laquelle nous nous trouvons, mais un engagement individuel envers des actes justes. Il faut donc remplacer l'éthique de la prospérité par une éthique de la Justice enracinée dans l'engagement personnel et le partage solidaire. Il n'y a de chance de progresser moralement que par la créativité des personnes en ce domaine et la responsabilité active de tous.

Les travaux, les lectures, expériences et réflexions qui sont à l'origine de cet ouvrage et en constituent la matière, s'étendent sur de nombreuses années. Entamé d'abord, dans les années 1960, comme un travail de thèse de philosophie à l'Université de Lausanne, sous la direction du professeur Daniel CHRISTOFF, ce livre a connu une première version qui a fait l'objet d'un colloqium doctum dans le cadre de cette université. Je souhaite remercier ici mon mentor, le Professeur Daniel CHRISTOFF, ainsi que le Professeur René SCHAERER, de l'Université de Genève, pour l'accueil très chaleureux qu'ils ont réservé à la toute première version de ce travail. Leur encouragement et leurs suggestions ont joué un grand rôle dans la forme que ce texte a finalement reçu, plus de vingt ans plus tard. Je ne puis évoquer la première étape de ce travail sans mentionner l'aide cruciale que m'a donnée, alors, ma famille aux Pays-Bas et en Suisse.

Le colloqium doctum de Lausanne eut lieu ce jour d'été de 1969 où l'être humain a mis pour la première fois le pied sur la lune. Une vision éthique de l'homme fut renversée ce-jour là, signe du changement des temps et des valeurs, dont ce texte est aujourd hui une expression. Les messages éphémères du destin ne reçoivent souvent leur sens que longtemps après leur manifestation historique.

Les aléas de ma vie personnelle et professionnelle m'ont empêché, pendant de nombreuses années, de poursuivre les recherches et les réflexions engagées, et le manuscrit a pour ainsi dire dormi, pendant deux décennies, avant que l'occasion se présente de le reprendre. J'ai pensé d'ailleurs, pendant de nombreuses années, ne jamais plus être en mesure de poursuivre ce travail, le domaine de mes intérêts et de mes travaux m'ayant entraîné vers des horizons parfois fort éloignés de la réflexion philosophique pure, celle-ci ne se poursuivant que sporadiquement durant mes heures de loisirs. 
Certes le souci de la réflexion éthique est demeuré constant au cours de ces années ${ }^{1}$ - on ne s'engage pas dans une telle réflexion sans en être définitivement affecté - mais je n'ai pas cru que le thème de l'obligation morale, tel que je l'avais alors abordé, ait pu avoir quelque liaison directe avec le champ de mes recherches ultérieures et de mes travaux.

C'est par le biais de la réflexion sur l'économie, l'Etat, la société d'abondance, et plus particulièrement sur le chômage et le sens du travail, qu'un lien a été établi entre les postulats des sociétés contemporaines et la réflexion éthique de base que j'avais entamée. L'occasion m'en fut donnée lors de nombreuses rencontres que $\mathrm{j}$ 'ai eues durant dix années de séjour à Maastricht, soit au "Centre européen Travail et Société», soit à l'Université du Limbourg, en particulier avec le Professeur Wil ALBEDA que j'ai eu le plaisir de côtoyer très souvent au cours de cette période.

Ces rencontres ont été suffisamment stimulantes pour que le besoin de reprendre le travail sur ce texte se fasse sentir. Un nouvel effort de réflexion devenait alors nécessaire, qui devait consister à décloisonner les diverses disciplines intellectuelles et les multiples champs d'action dans lesquels ma carrrière m'avait entraîné et d'introduire, comme lieu de rencontre, le thème même de la réflexion éthique que je poursuivais. Le manuscrit dormait peutêtre, mais sa vie se maintenait tout en se développant dans les questions récurrentes que je me posais. L'idée de reconsidérer dans ce sens le thème de l'obligation morale, et d'en faire un ouvrage nouveau proposant une thèse originale, m'est donc apparue alors comme suffisamment urgente pour $m$ 'y engager de façon définitive. J'ai ainsi repris le texte là où je l'avais laissé, le complètant considérablement, refondant la plupart des chapitres et terminant ainsi un travail abandonné trop tôt.

Je suis très reconnaissant à l'Université du Limbourg, à Maastricht, en particulier à sa Faculté d'économie, de me donner l'occasion de présenter ce travail comme thèse de doctorat, synthèse d'une longue maturation et oeuvre nourrie d'expériences très diverses. Le caractère transnational et interdisciplinaire de cette initiative est révélateur, d'une part de l'ouverture de cette jeune université aux transformations intellectuelles et académiques de l'Europe actuelle, et d'autre part de son approche pédagogique innovatrice quien a fait, en peu d'années, une université modèle. Je suis tout particulièrement reconnaissant au Professeur Wil ALBEDA, professeur de politique sociale, pour son encouragement et son soutien lors de la remise en chantier de ce travail, ainsi qu'au Professeur Dr. Edouard KIMMAN, professeur d'éthique de l'économie et des entreprises, à Maastricht et Amsterdam, pour le travail critique qu'il a bien voulu consacrer au bénéfice du manuscrit.

\footnotetext{
D'autres ouvrages que j'ai eu l'occasion de publier durant ce temps en témoignent: Le Royaume de l'homme, Essai sur la religion et la démocratie (1973); L'éducation créatrice (1975); L'homme et la vie: biologie contemporaine et éthique (1978) et Formation ef condition humaine au XXIe siècle (1991).
} 
Trop nombreux sont ceux qui, d'une façon ou d'une autre au cours de ces années, ont contribué à la réalisation de ce travail, pour que je puisse les citer tous. Je m'en voudrais cependant de ne pas mentionner tout spécialement Jean Van de Kerckhove et Alain Michel, pour l'aide directe qu'ils m'ont apportée dans la correction du texte, ainsi que mes proches et mes amis pour leur patience, en particulier pour la foi qu'ils ont gardée en cette entreprise en m'encourageant si longtemps de leur soutien. Qu'ils trouvent dans l'existence même de ce livre l'expression de ma gratitude. Qu'ils me pardonnent enfin les imperfections qui y demeurent et dont je demeure le seul responsable.

\section{G.F.}

$1969-1993$

Lausanne - Maastricht - Bruxelles 


\section{Chapitre 1}

\section{La question: Le retour de la philosophie morale}

La question du bien et du mal envahira cette fin de siècle.

Alain Etchegoyen La valse des éthiques

Parce que la vision libérale du monde est une vision morale, le libéral ne voit d'autre salut aux différents maux sociaux que dans un surcroît de moralisation.

François Ewald L'Etat providence

La question du bien et du mal est de tout temps une donnée fondamentale et permanente de la condition humaine. La réflexion éthique, elle, qui tente d'apporter une réponse à cette question pérenne, est sans cesse reconstruite, à chaque étape de l'histoire, parce qu'elle naît directement de l'expérience vécue. L'action humaine, en effet, - objet propre de la réflexion morale et en dépit de la prétention de celle-ci à l'universalité - $s$ 'inscrit toujours dans un espace précis et un temps limité, nourrie d'une culture particulière, incarnée dans une société distincte, marquée par un destin unique et soutenue de ses propres espoirs. C'est toujours à partir de leur situation historique que philosophes et moralistes élaborent les questions qui nourrissent leur réflexion et transforment leur image du monde. L'histoire de la 
philosophie le démontre. Il y a ainsi, dans toute tentative de formulation «intemporelle» de l'éthique humaine, une intention historique et politique.

Le présent travail est profondément marqué par cette tradition. Tout en s'enracinant dans l'héritage de notre civilisation et en s'appuyant sur les leçons des philosophies du passé, il tente de répondre, aussi courageusement que possible, par son contenu et l'angle de son regard, à certaines questions essentielles que la réflexion éthique soulève à notre époque.

Une remise en cause radicale de la notion éthique est à l'ordre du jour, et ceci pour deux raisons urgentes et apparemment contradictoires. La première concerne la prise de conscience d'une nouvelle liberté d'agir que l'homme semble avoir acquise par le pouvoir quasi illimité qu'il a conquis sur les choses et la nature, en raison de son ingéniosité technique et de la richesse des moyens que la croissance économique lui donne. La conjonction du progrès de la science et de la technologie, d'une part, et de l'accumulation des moyens matériels qu'il est possible aujourd'hui de réunir pour la poursuite de buts grandioses, de l'autre, a fait littéralement exploser le champ des possibilités d'action de l'humanité. Au nom de quels critères essentiels faut-il dès lors établir les priorités de l'action humaine quand ce n'est plus la finitude du possible qui détermine les choix humains? $\mathrm{Y}$ a-t-il une tradition éthique capable d'orienter l'homme au moment où l'expérience qu'il fait de sa liberté d'action l'envahit comme un éblouissement?

La deuxième est une prise de conscience inverse, mais causée par les mêmes effets. Pour la première fois dans l'histoire de l'humanité, en effet, l'homme prend conscience que son action a le pouvoir de menacer la survie de son espèce. Il est donc amené à se poser la question de l'être, - de son existence et de sa survie, en tant que personne individuelle, mais aussi en tant que société et civilisation - dans le jugement qu'il porte sur ses actes. Pour la première fois, l'idée s'impose avec évidence que le pouvoir conquis par l'homme sur la nature pour satisfaire ses besoins, et construire ainsi son bonheur, est aujourd'hui capable de mettre en danger l'existence même du monde et de l'espèce humaine, au moment même où les succès récents et les perspectives futures de la technologie nous envahissent et nous dominent comme une ivresse.

La question éthique prend dès lors une dimension entièrement nouvelle et s'impose avec urgence. L'éthique ne peut plus se permettre de considérer la question du bien et du mal au regard des seuls actes individuels, sub specie aeternitatis, dans un monde stable et un environnement plus ou moins maîtrisé, mais elle doit s'occuper de l'existence ou de la non-existence de l'humanité dans un monde en pleine évolution, à la fois libéré et menacé par l'homme. Plus que jamais, l'éthique ne peut plus être simplement individuelle, elle devient sociale et politique. C'est d'ailleurs une telle prise de conscience qui est à l'origine du renouveau de la philosophie politique en cette fin de siècle. 
L'orgueilleux pouvoir de l'homme, infiniment multiplié par la puissance économique et politique du nouveau Prométhée que sont devenues les sociétés contemporaines et leur haute technologie, a fait naître un sentiment de toute puissance qui entraîne l'humanité au-delà des fins temporelles qu'elle poursuivait. A force de développer et de perfectionner les moyens destinés à améliorer sa condition, l'homme apparaît dépassé par lui-même. Il ne semble plus capable d'arrêter le mouvement qu'il a mis en route. Ayant accumulé sans retenue les moyens matériels dont il pensait avoir besoin pour assurer sa survie devant une nature menaçante, en fonction de l'expérience des siècles antérieurs, l'homme du XXe siècle a produit les instruments d'une multiplication sans limites de ses propres moyens, au point que ceux-ci risquent de le détruire. A force d'ingéniosité et de travail créatif pour éloigner le danger du dépérissement de la race et la menace de l'indigence, l'homme doit faire face aujourd'hui au danger de sa propre disparition, emporté par le flot de ses oeuvres, écrasé par sa richesse, sa prospérité et ses succès.

Devant une telle évolution, qui apparaît à la fois comme une victoire et une menace, il semble urgent de trouver les instruments intellectuels permettant à l'homme de se maîtriser lui-même et d'orienter le pouvoir issu de sa propre raison vers un autre aboutissement que la mort. Par quelles questions peut-on le mettre en alerte? Comment élaborer les questions essentielles et les réponses pratiques qui lui permettront de comprendre que sa survie dépend uniquement de lui-même? Par quels biais lui faire admettre que son action, donc l'éthique qui l'inspire, est devenue non seulement l'objet de sa conscience personnelle, mais l'enjeu de sa vie dans le monde? Comment élaborer une nouvelle Welranschauung qui donnera à l'interrogation éthique la dimension politique nécessaire à ce nouveau défi de l'humanité?

A première vue, la philosophie morale apparaît mal préparée et inadéquate pour faire face à une telle interrogation. Elle ne trouve pas, dans ses traditions, les instruments nécessaires pour répondre à la question de la survie d'une espèce humaine libérée du besoin, car elle ne s'est jamais occupée que de la rectitude morale dans un monde durable et stable. En effet, "nulle éthique antérieure n'avait à prendre en considération la condition globale de la vie humaine et ... l'existence de l'espèce elle-même». ${ }^{1}$

C'est pourquoi une éthique susceptible de répondre aux questions de notre époque doit être en grande partie réinventée. Le regard fixé sur l'éternelle question du bien et du mal, nourrie de l'expérience acquise par l'histoire, et s'appuyant sur ses propres traditions, la philosophie doit proposer, pour aujourd'hui, une réflexion éthique à la mesure des enjeux de l'action humaine, individuelle et collective. C'est l'intention poursuivie dans ce livre.

${ }^{1}$ Hans Jonas, Le principe responsabilité, p. 26 


\section{La pensée économique, le bien, le mal et l'éthique de la prospérité}

La question du sens de l'action humaine dans la vie sociale est rarement poursuivie, de nos jours, à un niveau de réflexion qui semble correspondre à de tels enjeux. Dans les sociétés riches de cette fin de siècle, l'éthique sociale est avant tout, si ce n'est exclusivement, marquée par la primauté des valeurs matérielles. La production des richesses, leur distribution, le rôle des individus dans la création du bien-être, sont au centre des questions fondamentales que l'homme se pose au sujet de lui-même, de sa vie sociale et politique, et de son destin. Il semble qu'on ait trouvé le sens de la vie, découvert le cheminement sécurisant qui mettra fin à l'errance humaine dans I'histoire. La solution proposée est économique. Elle consiste à promettre à l'homme la satisfaction de ses besoins par la croissance d'une prospérité sans limites.

Cette évolution se manifeste d'abord dans la restructuration des domaines de la pensée. Les grandes questions dont traitait la philosophie politique, celles de l'Etat, du pouvoir, de l'égalité, de la liberté et de la Justice, font ouvertement partie, aujourd'hui, du domaine spécifique de l'économie politique, dans lequel les critères de quantité et d'efficience ont remplacé les notions de valeur et d'absolu. C'est en donc en termes d'économie, de bien-être et de croissance, ainsi que de compétitivité entre nations dans la course à la richesse et au pouvoir ${ }^{2}$ que se posent les problèmes de l'égalité entre les hommes, du rôle social de l'individu, des rapports de l'homme envers l'Etat, du sens de la Justice et de la liberté. Bref, de nombreuses interrogations sur l'homme, qui faisaient partie de la philosophie et de l'éthique, pour ne pas dire de la métaphysique, sont devenues des questions dont s'occupe l'économie, et ceci de façon quasi exclusive.

L'omniprésence de cet "économisme» a transformé le caractère de la réflexion morale en soumettant la problématique de l'éthique à des critères nouveaux. La science économique se propose, non sans arrogance mais non pas sans raison, comme une nouvelle éthique. Bien que développée avant tout, dans le passé, comme une science de l'action, elle prétend servir aujourd' hui de connaissance universelle de l'être humain et de son action, et de sagesse et de guide pour les gouvernants et les sociétés. Elle propose ses lois et ses principes comme des prescriptions infaillibles pour l'action, et tend à en étendre l'application sur tout le domaine de l'action politique. «La science économique touche au pouvoir: dans la mesure où elle s'intéresse à l'organisation de la société, elle est politique. ... (or) la politique économique fait remonter très vite aux options fondamentales de la théorie

2 Robert Reich, The Work of Nations 
économique, à la vision qu'elle fournit de l'homme et de la société. Toute théorie est implicitement normative»" ${ }^{3}$. L'économiste ne se contente donc pas de rechercher et de formuler des vérités, des principes ou des lois universelles concernant le fonctionnement matériel du corps social, de son système de production et de consommation; il vise l'action directe et, par elle, la transformation des sociétés. Ces «lois de l'économie», aussi relatives qu'elles puissent être en fonction des situations et de l'histoire dans lesquelles elles se développent et s'appliquent, ne visent pas à connaître, mais à faire. Elles sont prescriptives, tendent à s'imposer comme des normes, et visent à guider l'action. "Les idées des économistes et des politologues exercent une influence plus puissante qu'on ne le pense généralement. En vérité, elles mènent le monde» ${ }^{4}$. Quand on croit que ce monde est menacé, il est donc urgent de s'interroger sur les principes qui le guident.

Si la théorie économique dit ce qu'il faut faire, propose des fins à poursuivre et dirige les actions humaines, ses propositions prendront la forme et assumeront immanquablement la nature des propositions de l'éthique. De plus, en raison des succès indéniables et impressionnants dont l'action économique peut légitimement se vanter en cette fin du XXe siècle, et en fonction des preuves qu'elle a données, au cours de l'histoire récente, de sa capacité à transformer le monde et à satisfaire la plupart des besoins humains, les prescriptions de l'économie vont être dotées d'une force accrue de conviction. Il est difficile de mettre en cause la certitude qu'imposent les faits.

Durant ces dernières décennies, en effet, l'histoire de l'humanité a été transformée par les prescriptions de l'économie de façon beaucoup plus radicale que les éthiques traditionnelles n'ont pu le faire pendant des siècles d'enseignement, d'argumentation, ... d'admonition et de prédication. L'impact de l'application rationnelle des lois de l'économie sur les sociétés contemporaines est infiniment plus visible que celui du respect des normes morales et des croyances qui les soutiennent. En conséquence, au nom d'une efficacité indéniablement évidente, le champ de l'économie politique tend à se substituer progressivement au domaine de la réflexion politique et morale. Science par excellence des moyens, l'économie se propose aujourd 'hui comme une connaissance et une théorie des fins.

L'économie pénètre le champ de l'éthique parce qu' on lui reconnaît, par la force des choses, un rôle déterminant dans la constitution, le développement et la survie des sociétés. Cette reconnaissance implique toutefois l'acceptation d'une dimension et d'un rôle nouveaux des lois de l'économie;

3 Christian Stoffaès, Fins de mondes, p.246

4 John Maynard Keynes, cité in Stoffaës, op.cit., p. 246 
elles ne peuvent plus être considérées comme de simples recettes de l'action, indifférentes aux valeurs humaines, mais comme de nouvelles valeurs morales.

On parle de l'éthique économique, ou de l'éthique des affaires, comme d'un domaine nouveau de la réflexion humaine dont le but est d'éclairer l'action afin d'en améliorer le fonctionnement et les performances.

Il y a donc, par réaction, une forte tendance à «moraliser» la pensée économique et à la rendre plus conforme aux normes de l'humain, même si son champ demeure soumis à des valeurs uniquement quantifiables. On espère ainsi qu'une science économique soumise à l'éthique devienne plus acceptable, ses lois moins étrangères aux valeurs traditionnelles, son efficacité plus grande. Ainsi se développe une forme de moralisme économique qui se répand progressivement dans tous les domaines de la réflexion sur l'action. Cette tendance est éthiquement et pratiquement justifiée par le souci de s'assurer que tout, et si possible tous, contribuent au maintien et à la croissance de la prospérité générale. Car non seulement l'économie est efficace, mais elle peut prétendre, aujourd'hui, sur la base de ses succès, faire le bonheur de l'humanité. Non seulement la théorie économique donne une orientation à l'action, elle peut revendiquer le droit d'en déterminer la finalité. La richesse est non seulement souhaitable, elle est accessible si chacun contribue à la faire progresser. C'est par rapport à elle que sont élaborés les nouveaux critères quotidiens du bien et du mal de l'action humaine

C'est ainsi que la «richesse des nations», c'est-à-dire leur fondement et leur développement matériels, n'est plus considérée comme un instrument, un moyen susceptible de favoriser l'avènement du bonheur humain, elle est devenue, aux yeux de ceux qui en profitent, une finalité en elle-même. C'est dès lors vers elle que doivent conduire tous les actes humains; elle a été érigée en finalité ultime. Le bien-être matériel n'est plus seulement la condition minimale qui doit assurer le niveau de vie indispensable et la dignité matérielle des individus, afin de favoriser l'éclosion des vraies valeurs humaines, c'est lui qui est devenu la valeur suprême qu'il faut actualiser. La prospérité n'est pas au service de l'homme; c'est l'homme qui est progressivement mis au service de la prospérité.

Ce que nous appelons l'éthique de la prospérité est l'extrapolation logique de cette tendance dans le monde de la réflexion morale. Nous préciserons plus loin le contenu de cette nouvelle notion (voir Chapitre 7), mais disons d'emblée que c'est une éthique qui a pour but de donner un sens à toute action et de justifier moralement tous les actes qui contribuent au progrès du bien-être matériel de l'humanité. Il est donc bon, moral et «louable» que l'action de l'homme contribue à la croissance de la prospérité; il est mauvais, immoral et «condamnable» qu'elle y fasse obstacle ou qu'elle n'y contribue en rien. Le bien et le mal n'ont finalement de sens que par rapport à cette conquête de la richesse. 
Il est donc utile de se demander comment nous en sommes arrivés à cette idée, et ce qu'elle signifie pour la réflexion morale de notre temps.

\section{Une question en situation historique}

Comme souvent, dans l'histoire des idées, les réflexions des philosophes et les événements politiques se conjuguent pour entraîner la pensée à faire un saut qualitatif d'envergure afin de se situer d'emblée à la hauteur des nouveaux défis. Trois événements principaux se sont succédés, durant ces vingt dernières années, pour conduire la réflexion des philosophes et des économistes à ce stade de développement.

D'abord il y a un sentiment de crise, aussi durable qu'imprévu, qui affecte depuis bientôt deux décennies les économies occidentales. Il se manifeste dans divers domaines, entre autres dans le viol apparemment incontrôlable de l'environnement naturel et l'existence persistante d'un chômage échappant à toutes les politiques inventées pour le résorber. Cette crise a donc forcé la théorie économique à se renouveler et à s'étendre au delà du domaine strict de sa discipline scientifique. Quand le savoir sur les choses ne correspond plus à la vraie vie des choses, l'inquiétude s'installe et des questions fondamentales sont à nouveau soulevées.

Ensuite, nous avons été les témoins d'une renaissance, d'une diffusion rapide et d'une apparente prédominance d'un nouveau libéralisme que soutient le récent triomphe de l'économie de marché dans toutes les parties du monde.

Enfin, l'événement que représente la disparition du communisme comme alternative historique au capitalisme force ce dernier à devoir se justifier par lui-même, et non plus par la simple condamnation d'une doctrine qui s'y oppose. Le capitalisme libéral doit donc se justifier lui-même comme valeur humaine et sociale, et non plus se contenter de représenter l'alternative, «à visage humain", de l'anti-valeur qu'était à ses yeux le communisme historique. En ne vivant plus dans un monde divisé par une moralité bipolaire, - les bons d'un côté, les méchants de l'autre; le sacrifice et l'héroisme des uns pour combattre «l'empire du mal» de l'autre bord - il faut trouver dans la nature même du seul système qui apparemment survit la justification éthique de sa propre valeur. Il était plus facile de distinguer le bien du mal quand ils s'incarnaient chacun dans des régimes politiques antagonistes. La question ne se pose plus aujourd'hui dans ces termes; elle est renvoyée dans le champ de la réflexion personnelle et concerne les choix des individus et des collectivités humaines.

La situation historique ne nous permet plus de nous appuyer sur un bipolarisme simpliste dans lequel la justification de nos croyances et de nos actes nous était imposée par notre appartenance politique à un bloc ou à 
l'autre. Nous devons maintenant reconstruire notre propre système de pensée et recourir à notre propre engagement pour justifier nos choix. La liberté de mouvement que procure la fin de la confrontation idéologique est payée par le doute et l'incertitude. L'homme se retrouve donc plus seul qu'auparavant, et les communautés humaines moins certaines de la voie à suivre. Nous voilà donc ramenés à la question éthique fondamentale.

\section{Crise de l'économie ou crise de la théorie économique?}

La crise, tout d'abord, a été l'occasion d'une refonte profonde de la réflexion sur le fonctionnement du système économique, sur ses incohérences, sur l'illogisme de certaines de ses théories, sur l'inadéquation des politiques suivies, sur l'irrégularité du développement historique et la pertinence des cycles «kondratiéviens», sur les limites de l'approche keynésienne et des interventions étatiques, entrainnant une vague généralisée de privatisations, sur la crise de l'Etat providence, sur la révolution technologique et ses limites, sur le renouvellement du sens du travail humain, sur la performance et les compétences des individus, sur la relation traditionnelle du capital et du travail. La majorité des idées communément admises en ces domaines ont été mises en question, car les faits se sont développés de façon totalement indépendante des théories proposées. Devant de tels défis, les théoriciens ont souvent tenté, parfois en vain, de maintenir une certaine logique des idéologies dominantes dans un monde qui, soit ne les acceptait plus, soit continuait à fonctionner sans en tenir compte.

Et pourtant, la prospérité s'est maintenue et la croissance du niveau de vie s'est poursuivie dans cette tourmente intellectuelle; ce qui a fait dire à un critique de la pensée économique: «les peuples heureux n'ont pas d'histoire, .... de même, les nations prospères n'ont pas d'économistes" ${ }^{5}$. Ce décalage entre la réalité et son explication est peut-être une des raisons qui expliquent pourquoi la pensée économique se rapproche de plus en plus d'une pensée éthique. Comment, en effet, justifier rationnellement une science de l'action, quand l'expérience montre que la réalité dont elle s'occupe se développe et progresse indépendamment des lois qu'elle est supposée lui appliquer? On se justifie donc en s'appuyant sur les principes généraux plutôt qu'en s'accrochant à la formulation de recettes concrètes qui n'ont pas fait leurs preuves. Voilà pourquoi, si la vie économique se développe toute seule, selon un dynamisme qui lui est propre et sans intervention volontaire des pouvoirs qui la contrôlent, le libéralisme a beaucoup plus de chance d'être vrai que les théories du dirigisme!

5 Chistian Stoffaës, op.cit. p. 17 
Quelles que soient les causes qui expliquent les changements, les contradictions et les menaces du monde contemporain, il n'en demeure pas moins vrai que la raison demande à comprendre et qu'elle exige des principes sur lesquels elle puisse fonder une action favorisant le maintien et le progrès de la prospérité. Puisque cette dernière demeure l'ultime recours justifiant l'économie elle-même, il faut qu'elle soit fondée dans une éthique qui en rende compte. Le développement d'une pensée économique moralisante, au moment où la théorie économique se fait plus modeste parce que ses explications ont perdu la superbe de leur certitude, n'est pas le moindre des paradoxes qui résultent de la crise de ces vingt dernières années. Pourtant, tenter de le faire dans le seul cadre de la rationalité économique, sans en appeler aux fondements philosophiques qui en donnent la dimension morale et humaine, en en rattachant les principes aux grands courants de notre civilisation, est une tentative qui, à première vue, est destinée à échouer inévitablement. Il est dangereux, pour la théorie économique, de vouloir justifier ses principes par l'éthique, afin de compenser le manque de certitude des hypothèses qui en faisaient une science. Ce n'est pas le moindre signe de la confusion intellectuelle de notre temps.

\section{Prédominance du libéralisme et triomphe de l'économie de marché}

Le libéralisme se présente aujourd'hui comme la seule théorie politique et économique qui survive à la crise sans avoir trop perdu de ses plumes intellectuelles. On constate une résurgence des multiples écoles prêchant cette doctrine, appelant la grande «main invisible» d'Adam Smith à la rescousse pour bénir et justifier les nouveaux apologistes du marché. Il est vrai que le libéralisme peut être aussi bien une justification rationnelle qu'une échappatoire. En affirmant que les choses s'arrangent nécessairement d'elles-mêmes, on évite ainsi d'assumer le danger d'erreur qu'implique toute intervention volontaire. L'expérience Reagan aux Etats Unis, le Thatchérisme en Grande Bretagne et la perspective d'un grand marché européen, construit progressivement à grands coups de dérégulations, ont grandement renforcé les idées libérales tout en les renouvelant.

Une nouvelle éthique de la liberté s'est peu à peu dégagée de ces expériences, faisant miroiter des lendemains prospères par le seul truchement de la libre créativité des nouveaux agents de l'économie en prônant, en particulier, l'initiative individuelle, la primauté des entreprises privées, et la dérégulation généralisée. De telle sorte que de nombreux défenseurs du keynésianisme et de l'intervention publique se sont progressivement rapprochés d'autres idées classiques, telles celles de Schumpeter par exemple, à la recherche de justifications additionnelles pour vanter les avantages de la liberté et soutenir l'espoir de voir les entrepreneurs reprendre le flambeau défaillant de l'Etat. 
L'éthique de la prospérité représente une justification facile aux yeux de ceux qui ont renoncé aux théories passées, car toute proposition tendant à limiter la libre créativité des entrepreneurs va à l'encontre de cette morale qui a pour finalité la promotion d'un bien-être illimité. Tout ce qui peut contribuer à l'activité productrice de croissance et de prospérité doit être favorisé, et toute contrainte allant à l'encontre de ce but doit être moralement rejetée. Si les anciennes valeurs morales s'accommodent de ce principe, elles peuvent être retenues; si elles s'y opposent, elles doivent être mises de côté.

Mais il y a, dans le moralisme du libéralisme triomphant, un manque de fondement rigoureux et un relativisme qui sont loin de satisfaire l'esprit et qui demandent que soient recherchées des finalités humaines autres que celles qui y sont proposées.

La vieille question éthique sur la fin et les moyens de l'action humaine ressurgit dans ce débat. Il est difficile d'en rationaliser les arguments si le seul critère de la rectitude morale est celui de la performance quantitative, et si la seule mesure du bien se manifeste dans l'accroissement de la prospérité. Car la quantité n'a pas de limites rationnelles. Il y a immanquablement, parce que la croissance est ouverte sur l'infini, la tentation d'un nouveau «toujours plus» qui peut remettre en cause les valeurs établies. Est-il alors possible d'établir une morale qui puisse être acceptée par une telle liberté sans limites? Comment trouvera-t-elle les lois qui lui permettront de se dominer elle-même? Cette liberté quantifiée peutelle se donner une obligation? Ce libéralisme nouveau, dont le dynamisme historique est indéniable, fait ressurgir le besoin d'une réflexion morale qui fait appel à d'autres valeurs.

\section{La faillite du système communiste}

Le système communiste se proposait, depuis le début de ce siècle, comme une alternative historique au capitalisme, du moins à celui que le XIXe siècle avait vu naître. Son existence et son développement ont représenté, pendant des décennies, un défi incontournable. Le communisme a été contesté, combattu et intellectuellement condamné, parfois admiré, parfois rejeté, souvent reconsidéré, mais jamais ignoré. Les capitalistes euxmêmes ont dû en tenir compte et le considérer comme interlocuteur idéologique quasi-incontournable, en raison du rôle politique actif, intérieur ou extérieur, qu'il jouait dans la vie des sociétés et des pays occidentaux. Ce rôle fut reconnu partout, et ceci indépendamment de la confrontation entre les blocs idéologiques sur-armés qui se sont mutuellement et si dangereusement menacés durant la guerre froide. C'est pourquoi la prudence demandait - on ne sait jamais dans quel sens peut tourner le vent de l'histoire - de ne pas accorder au libéralisme, au capitalisme et aux formes de démocratie qui en étaient issues, une confiance absolue. Les diverses formes d'Etat provi- 
dence, de social-démocratie et de capitalisme social étaient justifiées par l'argument que tout en refusant le totalitarisme extrême du communisme, et en rejetant catégoriquement le manque de liberté individuelle qu'il entraînait dans les faits, il était possible de mettre en oeuvre, dans un socialisme modéré, certaines thèses fondamentales de cette éthique alternative que représentaient les sociétés communistes.

La faillite récente du communisme bouleverse complètement ce modèle, et renforce l'omniprésence, - faut-il dire la toute puissance? - de la pensée libérale. Elle consacre la primauté, si ce n'est l'hégémonie, d'un capitalisme qui peut prétendre assurer seul la prospérité des peuples de la terre entière. Les révélations fournies par les événements récents sur l'incapacité du système communiste à promouvoir la prospérité, et sur l'inefficacité des valeurs qu'il défendait pour satisfaire et garantir les besoins les plus simples des populations, sont un argument qui pèse. D'autre part, la volonté manifeste de tous les anciens pays à économie dirigée de s'engager aussi rapidement que possible sur la voie d'une économie libre de marché, ne fait que confirmer les penseurs libéraux et les défenseurs du capitalisme dans leur doctrine, et renforce leur croyance en la rectitude des valeurs de l'éthique de la prospérité. Il n'y a pas de vérité plus convaincante que celle dont l'efficacité et le succès sont historiquement palpables.

Mais cette rupture historique risque justement de réduire le contenu de l'éthique de la prospérité aux seules valeurs d'efficacité et de performance que prône le capitalisme le plus pur, faisant de cette morale une simple morale du succès, dans laquelle les recettes efficaces sont les seules lois et le rendement la seule norme. Il y a cependant trop de questions morales plus profondes qui se posent sur ce capitalisme triomphant pour maintenir le débat à ce niveau superficiel d'interrogation. Des questions fondamentales sont en jeu sur lesquelles nous devrons nous pencher avec plus d'attention dans cette réflexion. On ne change pas la carte du monde sans transformer radicalement les valeurs éthiques de ceux qui y agissent.

\section{Le désarroi de la pensée contemporaine}

Ces trois événements ont accéléré l'avènement d'un capitalisme tout puissant. Et tandis que celui-ci s'épanouit sans containtes dans une économie de marché où les seules règles admises sont celles qui garantissent la concurrence, l'histoire récente a eu pour effet de désarçonner les théories économiques et politiques bien établies. On ne sait plus très bien vers quelle théorie nouvelle se tourner. Car le principe théorique du «moins d'Etat», qu'il soit inspiré par la théorie économique ou soutenu par des visées politiques, ne constitue pas, à lui seul, une politique définitive et cohérente se justifiant par elle-même. Comment, en effet, élaborer des hypothèses qui puissent représenter la base d'une science susceptible de maîtriser une situation en perpétuel changement? Prôner le retrait et la neutralité d'un des 
acteurs principaux de l'action sociale ne constitue pas une doctrine susceptible d'orienter et de sécuriser l'action des acteurs qui demeurent.

Il n'est donc pas surprenant de rencontrer un immense désarroi dans la pensée de notre époque, surtout par rapport à ce que l'économie et les structures des pays prospères pourraient devenir à long terme. «Incontestablement, la crise est intellectuelle»"

Ainsi se manifeste le besoin d'une réflexion plus large et plus systémique, englobant d'autres éléments de la réalité humaine. Une simple justification scientifique des meilleures méthodes pour arriver à la prospérité ne semble en effet plus suffire. Christian Stoffaës le souligne clairement: «l'économie contemporaine a davantage besoin de philosophes que d'économètres»?

L'un des domaines particuliers où ce besoin de philosophie se fait sentir avec le plus d'urgence est celui de l'éthique, particulièrement au sein des nouveaux agents principaux de l'économie privatisée: les entreprises ${ }^{8}$. Devant la liberté nouvelle que l'ouverture des marchés et la dérégulation générale de l'économie apportent, il semble que les individus responsables, particulièrement quand ils se trouvent devant des décisions essentielles qui peuvent affecter les conditions de vie de grands ensembles, sont profondément désorientés par le manque de repères et de valeurs capables de rattacher leurs choix à d'autres fondements que l'unique souci du succès ou le seul accroissement du profit. Or ce dernier semble être la seule valeur objective du libéralisme le plus militant.

Certes, il y a quelque temps déjà que la croissance en tant que telle est mise en question et qu'on fait appel à une réorientation des finalités de l'économie. Les atteintes à l'équilibre naturel de l'environnement ont accéléré certaines remises en question. L'idée d'une croissance plus limitée, qualifiée de «soutenable» ou de «tolérable», est entrée dans le langage courant de nombreux futurologues. On comprend d'autre part que l'économie de marché ne peut, à elle seule, constituer une finalité humaine, puisqu'elle n'a pas d'autre but que de garantir la liberté d'action des partenaires qui y sont en concurrence pour augmenter les chances de prospérité. Or la garantie de la liberté des agents ne peut en soi constituer la finalité même de leur action. L'homme est libre pour agir; il n'agit pas pour être libre. Ainsi le marché, en tant que tel, ne peut constituer la finalité ultime de la politique économique. Il ne peut être, même dans la perspective d'une idéologie libérale, qu'un simple moyen. Certes on souligne que c'est assurément l'un des moyens le plus efficaces dans la poursuite de la prospérité, mais on ne peut pas aller au delà de cette affirmation. Le succès

6 Ibid. p. 10

7 Ibid. p.19-20

8 Voir par exemple: Alain Etchegoyen, La valse des éthiques; ou Institut de l'entreprise, L'entreprise et l'éthique. 
demeure donc la seule norme; la prospérité matérielle l'ultime objet de l'action. On n'échappe donc pas au besoin de chercher ailleurs, par d'autres efforts de la pensée humaine, ces normes de l'action qui seules justifient celle-ci et la rendent aussi juste et respectable en plus de sa performance et de son efficacité?

\section{Besoin d'une éthique universelle}

C'est justement le développement de cette éthique de la prospérité qui conduit la pensée morale actuelle dans une impasse. Car la détermination des actions humaines par la seule performance quantitative ne permet pas de répondre à toutes les questions que notre époque soulève. Il y a trop de questions urgentes, qu'elles concernent la survie de l'environnement naturel, la santé, l'intégrité génétique de l'espèce, l'équilibre psychique des individus, l'exclusion sociale des plus pauvres, qui ne trouvent pas de réponse satisfaisante dans les justifications que l'on propose en promettant un «toujours plus» de prospérité. En effet, quelle que soit sa prétention actuelle à rendre compte de toute la vie, particulièrement dans les sociétés d'abondance, l'éthique de la prospérité ne fait pas l'unanimité et ne peut prétendre à l'universalité, car elle ne sert pour l'instant de philosophie générale et d'école de sagesse qu'à ceux qui limitent leur projet humain aux valeurs qu'elle propose. Elle est avant tout une doctrine de l'efficacité. Mais si c'est à ce titre, et à ce titre seulement, qu'elle se propose comme une science de l'action, elle ne peut légitimer sa prétention à déterminer les fins ultimes de l'action humaine.

Comme instrument au service de l'efficacité, par son impact sur les motivations humaines, l'éthique de la prospérité ressortit directement au domaine de la morale. Elle ne peut cependant pas être proposée comme une théorie de morale générale. Car les thèses les plus élaborées sur l'efficacité de l'activité économique et leur analyse quantifiée ne peuvent pas remplacer les questions que tout homme se pose sur la finalité dernière de ses actions. Il est difficile d'admettre, en effet, que le sens de la vie humaine puisse être réduit à la performance de l'homo economicus!

Nous croyons que le développement de ce moralisme économique ne peut pas satisfaire entièrement l'interrogation humaine, ceci encore moins à notre époque que dans le passé, en raison même de la menace que la poursuite du bonheur matériel fait peser sur la survie de l'humanité future. Le principe responsabilité, dont parle Hans Jonas, demande une autre sagesse. Cette recherche de la sagesse, - éternellement renouvelée depuis ce jour inspiré où un premier homme inquiet prit conscience de lui-même en soulevant des questions insolubles, et tenta ainsi de saisir le sens de sa destinée, - semble s'accommoder difficilement de la pensée économique et se méfie donc des éthiques de l'efficacité qui tentent de tout justifier. Un retour à l'éthique fondamentale, qui replace l'éthique de la prospérité dans 
le cadre plus large de la philosophie et de l'histoire de notre civilisation, nous semble donc nécessaire. C'est non seulement nécessaire pour la satisfaction de l'esprit; c'est nécessaire à notre survie.

C'est pourquoi, sans vouloir renoncer à ce que l'économie et la pensée économique apportent au progrès humain, il nous paraît urgent de faire appel à une nouvelle interrogation philosophique sur la finalité de l'action humaine, en plaçant notre entreprise de réflexion dans le cadre d'une recherche éthique plus universelle. Le grand défi auquel doit faire face la pensée économique en cette fin de siècle, celui du fondement moral de son propre succès, ne pourra être surmonté qu'en replaçant les principes moraux qui lui sont indispensables dans une philosophie plus générale et plus universelle.

\section{Rétablir la philosophie dans le moralisme économique}

Alain Etchegoyen, dans le livre mentionné plus haut, exprime avec précision ce besoin de fondements plus solides qui se manifeste dans toutes les recherches d'éthique de l'entreprise, en disant: «il nous faut sortir de l'hypothétique pour s'assurer du catégorique»". Les termes utilisés sont significatifs: «s'assurer du catégorique», c'est-à-dire trouver une sécurité, si ce n'est une certitude, dans une formule qui ne soit pas l'objet changeant des fluctuations de l'action libre. Notre situation historique réclame "une éthique qui, par des entraves librement consenties, empêche le pouvoir de l'homme de devenir une malédiction pour lui»" ${ }^{10}$.

L'obligation morale est à nos yeux l'idée éthique qui peut répondre à ce besoin, car elle est à la fois une ouverture sur le devenir de l'homme et une contrainte qui puisse être librement consentie, donc une issue possible à l'inquiétude de notre temps.

Pour répondre à ce besoin, il ne s'agit pas de reconstruire une «moralité économique" nouvelle, pour remplacer celle qui nous apparaît comme déficiente, ni de proposer une moralité «extérieure», ou étrangère aux convictions individuelles, qui s'opposerait aux tendances dominantes et demeurerait de ce fait inefficace, mais de formuler une interrogation éthique qui permette de trouver, en l'homme lui-même et pour chacune des situations de sa vie, un ancrage moral capable de concilier le besoin de certitudes

9 Alain Etchegoyen, op. cit. p. 223-224

10 Hans Jonas, op. cit. p. 13 
fondamentales avec les aléas imprévisibles de l'exercice de la liberté. Ceci nous semble d'autant plus urgent que la liberté, présentée aujourd' hui dans le monde de la politique et de l'économie comme la seule loi susceptible de garantir le succès de l'action, peut être aussi considérée comme l'arme exterminatrice avec laquelle le nouveau Prométhée se menace lui-même. Situation tragique de l'homme qui doit, d'une part sauver sa liberté contre toute domination du monde extérieur pour être ce qu'il est, d'autre part maîtriser cette même liberté pour l'empêcher d'agir contre sa propre nature.

Face au désarroi de la pensée, et face à une éthique limitée par la seule finalité de la prospérité, nous nous proposons, dans cet ouvrage, de réfléchir sur l'obligation morale, "philosophiquement», et nous tenterons de montrer comment, par sa nature essentiellement humaine et morale, cette obligation peut proposer des réponses acceptables aux questions apparemment insolubles que soulève l'économie en cette fin de siècle. Mais parler d'obligation morale dans cette perspective ne veut pas dire qu'il faille replonger l'éthique dans un autoritarisme rigoureux, et reconstruire des comportements humains autour des morales de l'interdit. Ce n'est pas la quantité des lois et des règlements qui garantit la bonne marche des sociétés, c'est avant tout la capacité des sujets à maîtriser leur propre action. Montesquieu soulignait déjà qu'il ne pouvait y avoir de république sans la vertu des citoyens. Or, en une période où les idées libérales se répandent, où le libéralisme généralisé donne l'impression qu'il est possible de revenir au "tout est permis», il faut faire comprendre, par une réflexion éthique respectueuse de la liberté, que c'est la responsabilité seule qui permettra de «déréguler» la société sans la détruire.

L'analyse de la notion d'obligation morale, en remontant dans l'histoire de notre civilisation et de notre culture par les apports successif des philosophes et des moralistes, nous permet de développer une réflexion sur la place et les tâches de l'homme comme sujet responsable dans une société en pleine et totale évolution.

C'est à une nouvelle anthropologie et à une redécouverte de l'homme que nous convie cette tâche. Elle est causée par la nécessité de retrouver un sens à notre développement et à notre richesse. L'obligation morale, telle qu'elle nous apparaît, peut nous aider à fonder une nouvelle éthique sociale dans laquelle la valeur de chacun trouve sa complémentarité dans une entreprise commune. A la place de l'isolement que crée l'individualisme, nous nous attachons à la solidarité que crée la responsabilité des personnes. L'intervention éthique dans un monde de liberté ne dit pas ce qu'il faut faire, elle montre que l'homme est responsable de tout. La société future a besoin qu'on affirme le rôle essentiel de l'obligation morale parce qu'elle veut respecter la liberté.

Cette tentative a pour but de redonner un fondement ontologique à l'action humaine, pour la libérer d'une finalité trop exclusivement quantitative. Ceci devrait nous conduire à l'affirmation de l'existant sur le 
performant, de l'être sur l'avoir, du devenir sur le déclin, et de la création sur la mort, tout en respectant la liberté. C'est pourquoi il faut réintégrer l'éthique dans l'être. Il ne s'agit pas de proposer ici une nouvelle théorie morale de l'économie, ni de construire des formules éthiques ou des recettes pratiques pour les chefs d'entreprises, ni de mettre en question les acquis de la liberté et les bienfaits de la prospérité contemporaine; il convient bien plutôt de proposer une réflexion nouvelle, fondée sur la tradition philosophique de l'Occident, qui puisse contribuer à enrichir la pensée économique afin qu'elle dépasse ainsi les limites du monde clos de l'efficience et de la performance dans lesquelles elle risque de s'engloutir. Vouloir échapper à un monde fermé sur lui-même ne signifie pas vouloir le détruire. Critiquer la performance au nom de l'éthique ne veut pas dire qu'il faille rechercher le salut dans l'indolence et la passivité, mais souligne l'impossiblité de faire des valeurs d'une seule sphère de l'action les lois de toute la vie humaine. L'économie ne doit pas tenter de s'imposer à toute la vie morale. C'est ainsi que la performance ne peut pas être le juge ultime des actions humaines.

Mais cette interrogation, qui trouve son origine dans les problèmes pratiques de notre époque, doit également interpeler le philosophe. Lui aussi doit comprendre que le monde idéal des concepts ne peut suffire et que sa réflexion doit - autre obligation tout aussi catégorique, - se plonger dans la mêlée conflictuelle de l'histoire pour atteindre la profondeur réelle de l'existence. C'est le sens même de toute philosophie morale et de toute réflexion éthique. 


\section{Chapitre 2}

\section{Historique de l'obligation morale}

Lorsque j'emprunte mes idées à des philosophes anciens ou contemporains, ce ne sont pas seulement des idées que je leur dois; je leur dois tout, c'est-à-dire l'éveil fondamental à la philosophie, sans lequel des pensées apprises ne sont que néant. Je leur dois ce que je suis et ce que je peux devenir.

Jeanne Hersch L'illusion philosophique

Par respect pour la logique philosophique, une étude de l'obligation morale dans l'histoire de la civilisation occidentale devrait s'ouvrir, en raison de la place que ce problème tient dans la pensée contemporaine depuis Kant, par une analyse de la théorie kantienne du devoir et remonter dans le temps, à partir de cette analyse, pour donner aux théories plus anciennes un nouvel éclairage. On sait, en effet, que la notion de devoir n'a pu être analysée pour elle-même qu'au moment où Kant en a retiré toute connotation de finalité et l'a libérée, - devrions-nous dire purifiée? - de toute implication instrumentale et de toute détermination matérielle. En établissant l'obligation dans l'a priori de la raison, Kant la dégageait des conditions historiques dans lesquelles elle se révélait, et permettait ainsi d'en faire une question permanente de la philosophie.

Nous ne pensons cependant pas qu'il faille renoncer à la chronologie des développements qui ont justement permis l'aboutissement kantien. Ce n'est pas tellement en relation avec la théorie de Kant que nous croyons nécessaire 
de remettre l'obligation au centre de l'interrogation morale mais, comme nous l'avons démontré, en raison d'une problématique et d'une inquiétude actuelles, à savoir: comment donner un sens à l'action libre des sujets quand ceux-ci se trouvent menacés d'étouffement ontologique parl'environnement social.

L'interrogation philosophique est souvent tentée de se situer dans l'intemporalité et de formuler des questions qui ne s'appuient que sur la seule rigueur de la raison. Mais une telle attitude demande de résoudre préalablement une autre exigence fondamentale de la philosophie, à savoir: celle de la nature et du champ de la question philosophique elle-même, comme a tenté de l'indiquer Heidegger'. En soulevant ce préalable, nous soulignons, comme la philosophie du XXe siècle l'a si souvent fait, que les questions authentiquement philosophiques sont de nature réflexive, et qu'elles constituent une démarche de l'esprit dans laquelle l'homme luimême, en sa qualité de sujet pensant et agissant, est le véritable enjeu des problèmes qu'il soulève. Il ne peut donc y avoir de philosophie que par le sujet agissant et engagé. Ce retour au sujet dans la philosophie contemporaine se situe donc pleinement dans la ligne de ce travail.

Il existe, dans toute question philosophique parce qu'elle est réflexive, une interaction entre l'être et le faire, entre l'immuable et le devenir, entre l'universel des principes apodictiques et l'ambiguité concrète d'une situation particulière. La condition philosophique consiste à reconnaître qu'un point de départ n'est qu'un seuil de passage où le philosophe est conduit et porté par sa propre histoire. Ma pensée n'est jamais mon unique pensée, elle implique toujours celle d'autrui: celle du passé dont elle naît; celle du présent par laquelle elle prend conscience d'elle-même; celle du futur dans laquelle elle trouvera son aboutissement et son dépassement.

Il ne serait donc pas possible de résoudre l'ambiguïté de la morale économique de cette fin du XXe siècle sans faire référence à l'histoire. Mais nous le ferons philosophiquement, c'est-à-dire en tentant d'enraciner notre réflexion dans une perspective transcendante et intemporelle qui, se reportant à l'histoire dont elle est issue, lui donne un sens particulier.

Car il n'y aurait pas d'histoire et de présent assumés, mais un simple enchaînement chronologique d'antécédents et de conséquents sans signification aucune, si la réflexion ne venait pas reconstituer le déroulement des choses et des idées et lui donner un sens qui enrichisse notre présent. Mais il n'y aurait pas de philosophie non plus si, devant les interrogations contemporaines, l'histoire ne s'imposait pas et ne demandait pas à la philosophie de se renouveler, de se dépasser, et n'exigeait pas une perpétuelle remise en question de l'homme et de sa condition. C'est pourquoi l'homme n'est

1 Martin Heidegger, Introduction à la métaphysique, en particulier le Chapitre I: «La question fondamentale de la métaphysique». 
finalement assujetti ni à l'histoire ni aux principes immuables d'une vérité absolue. Il sauve ainsi sa liberté.

Poser le problème central de la morale en termes d'obligation, ou de devoir, est l'apanage de la philosphie post-kantienne, puisque c'est par Kant, «notre plus ancien contemporain philosophique ${ }^{2}$, que cette question a acquis les dimensions de l'a priori et du formalisme dans laquelle nous la comprenons aujourd'hui. Mais en raison de la dialectique qui caractérise la relation de la philosophie et de l'histoire, c'est la question du devoir qui va nous permettre de donner un sens nouveau et une orientation particulière à l'histoire de la philosophie et de la réflexion morale dont il est issu. Ainsi l'absolu du devoir et l'historicité de sa révélation vont s'éclairer mutuellement.

La première section de ce Chapitre: Le développement de la conscience et la révélation du devoir, présente trois étapes de l'histoire de la pensée au cours desquelles les constituants principaux du devoir ont été révélés et précisés. D'abord Socrate qui, par son ironie, a su mettre en cause le sujet, conduisant à la conscience de soi par le «connais-toi toi-même». Le Christianisme, ensuite, qui, en rattachant la conscience individuelle à l'existence d'un Dieu personnel, a favorisé la découverte de l'intentionnalité et a enraciné la conscience dans l'absolu. Kant, finalement, qui a constitué le devoir comme le fondement même de la morale et de l'autonomie de l'homme. Conscience de soi, intentionnalité et révélation de l'homme par le devoir, trois éléments indispensables de l'affirmation du sujet.

La deuxième section: L'explication de l'obligation et la dissolution du devoir, est consacrée à des auteurs qui ont contribué à l'évolution de l'idée d'obligation dans la période post-kantienne, mais qui, en tentant d'expliciter l'obligation par l'introduction d'un contenu dans l'a priori kantien, ont conduit à la dissolution de la notion d'obligation.

La troisième section: L'obligation aujourd'hui, tente de donner une courte synthèse de la situation actuelle et indique comment cet héritage historique s'exprime encore dans les hésitations éthiques de la philosophie politique d'aujourd'hui.

2 Gerhard Krüger, Critique et morale chez, Kant, Introduction, p. 27 


\section{Le développement de la conscience et la révélation du devoir}

\section{L'obligation socratique et la morale antique}

On ne retrouve pas, dans la morale antique, une doctrine constituée ayant traité du problème de l'obligation tel que nous le connaissons aujourd'hui. Si Platon, dans le Criton, aborde le problème du devoir, il s'agit d'un devoir très précis, bien déterminé dans son contenu, et non pas du devoir pris en lui-même. L'auteur du dialogue, en effet, n'abstrait pas la notion de devoir et ne la distingue pas de la fidélité aux lois de la cité, fidélité concrète et précise au nom de laquelle Socrate accepte de mourir et sans laquelle, surtout, aucun ordre social et aucune justice ne seraient possibles. $\mathrm{Si}$ Aristote, pour sa part, mentionne les notions de «il faut» ou "on doit», il ne les sépare jamais de leur objet direct et de la finalité dans laquelle il voit le fondement de toute action morale. L'expression grecque utilisée par Aristote, to déon ${ }^{3}$, signifie à la fois ce qu'il faut et ce qui convient ou ce qui est opportun, soulignant parfaitement les notions d'instrumentalité et de finalité qui y sont impliquées. "Bien loin que ce soient des catégories de l'obligation morale», écrit Léon Robin, "ce sont plutôt des cadres pour l'appréciation du bon usage du plaisir» ${ }^{4}$. De même dans la morale stoïcienne, même si elle admet l'existence d'actions absolument bonnes dépassant les finalités rationnelles, elle ne demande pas des actions vertueuses par devoir, mais toujours en vue d'une fin, celle du bien. Quand aux règles pratiques de conduite que cette morale propose, les kathèkonta, il n'est pas possible de les traduires par devoirs, et «les termes de fonctions ou de conduites convenables semblent mieux venus»s.

\footnotetext{
3 Aristote, Ethique de Nicomaque, Livre premier, II, 2

4 Léon Robin, La morale antique, p. 110

5 Jean Brun, Le stoïcisme, p. 115
} 
L'idée d'une obligation, dans la philosphie antique se présente toujours sous la forme d'une appropriation des moyens à appliquer et des voies à suivre pour parvenir au bien et au bonheur. «Les Grecs n'ont jamais conçu de vie morale, si élevée fut-elle, qui ne dût avoir le bonheur pour récompense» ${ }^{6}$, quelle que soit l'élévation et l'idéalisation de l'idée qu'ils ont pu se faire de ce bonheur. Bonheur individuel, certes, et non pas social, aussi aucune notion utilitariste du "maximum de bonheur» pour le plus grand nombre n'est à attendre de leur part.

Dans cet ensemble de morales que nous a laissé la philosophie antique, une perspective nouvelle se dégage: l'approche de Socrate. Certes nous ne devons pas tenter de retrouver dans l'apport de Socrate une doctrine constituée en système, ce qui serait paradoxal pour un philosophe qui n'a jamais écrit, mais voir plutôt dans sa démarche le début d'un mode de pensée réflexive qui a contribué à construire la philosophie conduisant à l'obligation. Son originalité, en effet, ne se trouve pas dans le contenu de la morale qu'il a pratiquée et enseignée, mais dans la connaissance nouvelle de cette morale à laquelle il a convié ses disciples.

\section{L'approche socratique}

Le point de départ de Socrate est une réflexion critique sur les deux disciplines qui se partageaient les esprits d'Athènes, la physique et la sophistique. Les physiologues tentaient de comprendre le monde en ramenant la réalité à de grands principes explicatifs généraux, sans recourir pour cette explication de la nature et de l'être à des mythes venant des puissances surnaturelles. Une telle entreprise apparaissait sacrilège aux yeux de Socrate, esprit profondément religieux. Aussi il distinguait dans la réalité deux domaines: d'un côté les choses humaines; de l'autre les choses divines. L'homme ne pouvait connaître rationnellement que les premières. Les choses divines, elles, c'est-à-dire la formation du monde, son destin, sa finalité, faisaient partie du domaine réservé à la connaissance des dieux. II y avait une tentation de la raison dans laquelle Socrate ne voulait pas tomber, aussi il rejetait l'objet de la recherche des physiologues. Mais cela ne signifiait pas qu'il allait renoncer à tout ce que cette physique avait apporté à la connaissance humaine: les principes rationnels, la forme de la pensée, la méthode. Cela va entraîner une prise de conscience du besoin de l'esprit humain d'introduire une unité et une harmonie dans ses propres connaissances, de reconnaître l'existence d'une vérité impersonnelle, universelle, distincte de l'opinion et de l'imaginaire, bref l'idée abstraite de la science. Socrate rejette l'objet de la physique, mais il en conserve la méthode et la démarche scientifique.

6 Léon Robin, op. cit., p. 171 
La critique socratique de la sophistique y distingue une erreur de même nature, mais inversée. L'objet de la sophistique correspond bien à ces choses humaines que l'homme peut connaître sans violer la loi divine, mais la méthode que suivent les sophistes ne conduit à aucune connaissance réelle, elle n'est qu'une manipulation de l'opinion. Certes les sophistes ont raison de se limiter aux choses humaines et de vouloir rendre les hommes capables de bien parler et de bien agir, de bien administrer leurs affaires et celles de la cité, d'être utiles aux autres et à eux-mêmes, mais le moyen qu'ils utilisent pour atteindre ce but est inefficace. La seule pratique ne suffit pas, car elle confond le moyen et le but. Ceci nous rapproche directement de la situation actuelle et du «moralisme économique» contemporain que nous avons stigmatisé. Si nous disons aujourd'hui que l'économie ne peut être sa propre finalité, Socrate lui, affirmait déjà: l'art ne suffit pas à l'art, il ne peut être vraiment efficace que dans la mesure où il est fondé sur une connaissance, car «chacun de nous vaut en fonction de ce qu'il sait précisément».

D'un côté Socrate rejette l'objet des physiologues, car ces derniers avaient appliqué la forme de la science à un objet qui la dépasse, et il corrige les sophistes qui n'appliquent pas cette connaissance à l'objet qui la comporte et qui l'exige. A partir de cette double critique, Socrate construira une approche philosophique propre qui consistera à combiner ce qu'il $y$ avait de valable et de certain dans chacune de ces disciplines, à savoir l'approche scientifique par la connaissance de l'universel, et le souci exclusif des choses humaines. La science que propose Socrate est donc essentiellement une science morale.

\section{Le «connais-toi toi-même»}

La connaissance qui est ainsi capable de fonder un art et une pratique authentiquement humains sera dès lors la connaissance de l'homme luimême. Le «connais-toi toi-même», la maxime que le Dieu de Delphes a laissé aux hommes, en devient le premier principe.

Mais la maxime de Delphes peut être interprétée de deux façons. Si elle est interprétée selon les principes scientifiques des physiologues, elle apparaîtra comme une exhortation à découvrir en nous ce qu'il y a de général, c'est-à-dire la nature commune à tous les individus, nature servant de fondement à l'art et de critère pour la convenance de nos actions. Mais une telle science est apparue très vite limitée aux yeux de Socrate, car l'homme moral est beaucoup plus complexe que les concepts par lesquels il s'exprime à lui-même sa propre nature, et la science de l'homme demeure limitée, inachevée. Socrate a été le premier à prendre conscience de la complexité et de la profondeur insondable de l'homme moral: «Je cherche», dit-il, «si je suis un animal plus compliqué que Typhon et plus méchant, ou si ma nature est simple et participe à la divinité»? 
C'est pourquoi de nombreux dialogues socratiques n'aboutissent pas et finissent en soulevant plus de questions qu'ils n'en résolvent. C'est en particulier le cas quand le thème du dialogue est la sagesse et le «connaistoi toi-même». Tandis que, dans le Premier Alcibiade, Socrate avait reconnu que le uconnais-toi toi-même» était le fondement et la condition sine qua non de toute morale concrète et efficace, il remet cette affirmation en question dans le Charmide lors de la discussion avec Critias sur l'utilité de la sagesse comme connaissance.

La première conclusion à laquelle Socrate et Critias parviennent, ayant analysé la fonction de la sagesse dans la connaissance de l'action, est de reconnaître que la sagesse, la connaissance de soi-même, n'est pas un savoir comme les autres savoirs; tandis que ceux-ci portent sur d'autres choses qu'eux-mêmes, seule la sagesse est le «savoir des autres formes du savoir et elle l'est d'elle-même»" ${ }^{8}$. Dans ce cas, fait remarquer Socrate, elle doit être également le savoir du non-savoir. Mais comment envisager, dès lors, un savoir qui ne soit pas le savoir de quelque chose, et dans quelle mesure ce savoir peut-il affirmer ou nier que les autres savoirs sont réellement des savoirs? Il faut donc en conclure qu'une telle sagesse se révèle d'aucune utilité pratique. Cette connaissance de soi ne peut donc nous conduire à une activité réussie et nous donner le bonheur. Il faudrait pour cela avoir une autre connaissance, un autre savoir unique, «celui qui se rapporte au bien et au mal». Mais alors, quelle serait la valeur de l'exhortation du Dieu de Delphes? Ne serait-elle plus vraie? Si le «connais-toi toi-même» équivaut à "sois en sagesse», alors la sagesse ne peut être confondue avec la connaissance du bien et du mal. «En conséquence, si ce dernier savoir nous est utile, la sagesse devra être autre chose qu'utile»?.

Ainsi donc, en fondant la recherche de la sagesse sur l'inscription de Delphes, Socrate arrive à la conclusion que cette sagesse ne peut avoir aucune utilité morale. Quelle est donc, dès lors, la signification réelle du "connais-toi toi-même"?

Le raisonnement du Charmide va nous mettre sur la voie. Si cette sagesse fondée sur la connaissance de soi est un savoir qui n'a pas d'objet propre, si elle n'est pas un savoir de quelque chose, elle ne peut être que la révélation du sujet qui connaît, c'est-à-dire de cette réalité qui ne peut être à la fois l'objet et le sujet de sa connaissance. Cette révélation ne vient pas d'une conclusion à laquelle aboutirait le dialogue, puisque celui-ci n'en connaît pas, mais du doute même dans lequel Socrate laisse son interlocuteur. Car c'est cela même que vise Socrate; non pas des vérités nettement formulées mais, à l'occasion d'une recherche sur le courage, la vertu ou la sagesse, l'éveil d'un esprit à la réflexion, un éveil à la conscience, un appel à la

\footnotetext{
7 Phèdre, 230, a

8 Charmide, 166, c

9 lbid., 174, d
} 
responsabilité envers ses propres actions, une ouverture, - telle que nous l'exprimerions en langage de notre époque -, vers la prise de conscience de la liberté.

Alors que, dans l'Alcibiade, Socrate se limitait à une présentation scientifique du «connais-toi toi-même», qui pouvait conduire à l'utilitarisme moral, faisant de la connaissance de soi-même l'instrument de l'amélioration de l'homme, la démarche du Charmide ouvre des perspectives tout à fait différentes. Il s'oppose en effet à une connaissance de la nature humaine qui se bornerait à n'être qu'un inventaire des qualités et du fonctionnement humain, car elle pourrait se prolonger en une technique de manipulation conduisant à un pouvoir sur l'homme par la connaissance de l'homme. Au contraire il propose une prise de conscience de soi qui s'ouvre à la liberté.

Il y aurait certaines extrapolations à faire, à partir de cette prise de conscience, sur les analyses des comportements humains qui sont aujourd'hui proposées par la science économique, et qui visent à une maîtrise des réactions humaines pour satisfaire la cohérence des théories de l'action. Socrate s'y opposerait avec très grande vigueur.

Cette approche socratique conduit enfin à la révélation de la subjectivité. Cette subjectivité, chez Socrate, n'est cependant pas considérée comme le point de départ d'une connaissance, à la manière du cogito cartésien, mais comme une exhortation à une prise en main individuelle de sa propre vie, l'affirmation d'une condition essentielle de toute vie morale et humaine. Elle est la condition même de cette philosophie dont parle le Phédon qui recommande aux âmes «de s'assembler, de se ramasser sur elles-mêmes, de ne se fier à rien d'autre qu'à elles-mêmes; quel que soit l'objet, en soi et par soi, de leur pensée quand elles l'exercent d'elles-mêmes et par ellesmêmes ${ }^{10}$.

Avec Socrate la pensée humaine fait un saut. Elle se détourne des choses, de l'extériorité dans laquelle elle craignait se perdre, pour se retourner sur elle-même, se saisir et prendre conscience de soi. Dès lors, la formulation du message socratique ne peut être à l'indicatif, comme sont formulées les vérités objectives, mais elle doit contenir une invitation, un ordre, et être «à l'impératif». Socrate ne dit pas que l'aboutissement de sa recherche est le moi dont il donnerait une définition et dont il ferait l'objet d'une connaissance; il présente au contraire cette révélation du moi comme une obligation: c'est l'impératif: «connais-toi toi-même», qui constitue le coeur et l'essence même du message socratique.

La pensée grecque n'a jamais pu imaginer une connaissance qui n'ait pas d'objet; c'est pourquoi l' impératif delphique n'est pas une réelle connaissance, et ne peut fonder la vertu. Socrate n'en fait donc pas un devoir pratique, mais

10 Phédon, 83, a 
le présente plutôt comme une invitation, une sorte de promesse, qui se situe davantage dans le domaine de l'être que dans celui de l'agir. Cet impératif suggère plutôt: «sois toi-même», que «agis par toi-même». La philosophie de Socrate doit donc être considérée davantage comme une invitation à l'existence que comme une ouverture vers l'autonomie morale.

Cette autonomie morale ne pourra en fait être pleinement révélée qu'au moment où l'homme, conscient de ce qu'il est, se trouvera face à une loi morale qui s'impose à lui par l'absolu de Dieu. C'est pourquoi nous verrons que le «connais-toi toi-même» ne prendra toute sa signification dans notre civilisation qu'avec la christianisation de l'hellénisme. Mais la découverte de l'authentique autonomie morale aura été préparée par l'intuition de Socrate. Elle est à nos yeux le premier jalon posé par la pensée occidentale sur le chemin d'une théorie complète du devoir.

Toutefois la morale socratique ne se présente pas comme une morale du devoir. Il s'agit maintenant de comprendre pourquoi.

\section{La finalité dans la morale antique}

Pour Socrate, comme pour tous les Grecs, la finalité de l'action est le fondement réel de sa moralité. Il suffit donc de bien connaître cette finalité pour agir moralement, comme par automatisme. Il suffit de connaître le bien pour le faire, car pour le sage, connaître et agir ne sont qu'une seule chose. C'est d'une telle conception de la sagesse morale que naîtra le principe socratique: "nul n'est méchant volontairement" ".

Pour agir moralement et faire le bien, il suffit à l'homme de connaître le bien et la vertu, et ainsi de parvenir au bonheur. Le sage est celui qui connaît; le méchant celui qui ignore. S'il existe une obligation dans la morale socratique, c'est une obligation de convenance, fondée sur le bonheur que poursuit le sage par la connaissance du bien et de la vertu. Cette vertu elle-même est considérée bonne parce qu'elle est utile, car «tout ce qui est bon est utile». Cette conception utilitaire de la vertu fonde «l'obligation d'être vertueux» non pas sur la bonté in trinsèque de la vertu, parce qu'elle recèlerait en elle-même une partie du bien, mais tout simplement parce qu'elle est la voie qui permet au sage de parvenir au bonheur. L'obligation se démontre ainsi par son utilité.

Il y a certes une certaine ambiguité dans la position socratique, entre l'utilitarisme de la vertu et son appel au «connais-toi toi-même» qui ouvre le champ de la subjectivité. Car si l'impératif delphique se révèle moralement inutile, comme cela est montré dans le Charmide, il n'en est toutefois pas

11 Timée, 86, e; Protagoras, 345, d 
rejeté. Sans donner l'impression de bien savoir quelle place lui donner et quelle fonction il remplit exactement, Socrate le considère comme essentiel à la connaissance des choses humaines auxquelles il a limité le pouvoir de la science. Dans cette perspective, Socrate nous montre que la sagesse totale se trouverait au delà des fins pratiques que peuvent atteindre les vertus «utilitaires». Mais ne faudrait-il pas voir dans cette subjectivité le point où la connaissance des choses humaines et celle des choses divines pourraient se rencontrer, à partir duquel il serait possible de fonder une authentique métaphysique de l'homme?

Socrate ne semble pas être allé plus loin dans la direction qu'il avait entrevue, et il faudra attendre encore de longs siècles pour que la philosophie découvre, dans la réflexion sur le sens de cette subjectivité, les fondements d'une telle métaphysique de l'homme. Bien qu'il semble avoir entrevu cette dimension infinie de l'homme, il semble que Socrate n' ait pas fait le pas qui lui aurait ouvert le champ mystérieux d'une connaissance rationnelle de luimême. Par respect pour le divin, Socrate avait voulu limiter la raison humaine en la restreignant à la connaissance des choses humaines, mais il n'a peut-être pas vu qu'il lui ouvrait ainsi un champ beaucoup plus large et beaucoup plus profond parce que touchant directement au véritable mystère humain, dans la profondeur de la liberté où se manifeste le devoir. Socrate est parvenu au seuil du domaine de l'obligation, mais il semble qu'il n'ait pas eu l'heur d'y pénétrer.

Ses successeurs, et principalement Aristote, enrichis par la méthode construite en vue de la science morale, édifieront une nouvelle philosophie de la nature qui sera post-socratique dans sa méthode, mais pré-socratique dans son intention. La distinction entre le domaine des choses divines et des choses humaines s'estompera. Bien plus, la connaissance des choses humaines sera directement fondée sur les principes des choses divines. La connaissance de l'homme ne se distinguera plus de la connaissance du monde. Quand Aristote écrit que les leçons de Socrate "portaient exclusivement sur les choses morales, et nullement sur la nature entière» ${ }^{12}$, il lui reproche d'avoir ainsi déraciné, pour ainsi dire, la connaissance des actions humaines de leur fondement ontologique. Les philosophies postérieures à Socrate, en effet, ne conçoivent pas la morale comme se suffisant à elle-même; elle nécessite un fondement cosmologique ou métaphysique dans lequel l'homme, étant situé à sa vraie place dans l'ordre de la nature et la hiérarchie des êtres, la connaissance de sa vertu se déduit de la connaissance de son être.

Mais comme une telle métaphysique sera elle-même construite sur les principes rationnels établis par Socrate pour sa philosophie exclusivement morale, c'est sur le principe d'utilité, c'est-à-dire sur le principe du Bien et de la finalité, que les métaphysiciens ultérieurs construiront d'une part

12 Aristote, Métaphysique, A, 6, 987, b 
leur théorie de la nature et d'autre part leur théorie morale. Aristote est certainement l'exemple le plus typique de cette tendance. Sa morale est construite sur la connaissance préalable de la fin dernière et du Bien suprême; connaissant ce Bien, "comme des archers qui ont sous les yeux le but à atteindre, nous aurons des chances de découvrir ce qu'il convient de faire" ${ }^{13}$. La loi morale est donc "ce qu'il convient de faire», et sera formulée comme une convenance, comme un instrument et un moyen pour atteindre une fin déterminée d'avance dans laquelle l'homme trouvera un bonheur assuré. "Ce thème», écrit Jacques Maritain, «deviendra classique pour des siècles dans la tradition philosophique occidentale, jusqu'à la révolution kantienne» ${ }^{14}$,

\section{La perfection stoïcienne}

Nous avons indiqué plus haut que la notion stoïcienne kathèkon, que Jean Brun propose de traduire par l'expression «conduite convenable», ne peut être assimilée à la notion de devoir. En effet, ce que Cicéron appelle l'officium n'exprime en fait que ce qu'il convient de faire dans une situation donnée, en raison des conditions concrètes de l'action que l'officium régit. Pourtant cette notion n'est pas l'unique notion qui a été développée par la pensée morale stoïcienne.

Cicéron écrit: «Nous pourrions appeler, je pense, le devoir "parfait» l'action droite, puisque les Grecs l'appellent katorthôma, tandis qu'ils appellent l'acte ordinaire qui nous occupe kathèkon. Et ils les définissent ainsi: ce qui est droit, c'est cela le devoir «parfait», mais ils nomment devoir "moyen", cette action qu'une raison "probable» peut justifier» ${ }^{15}$. Or si nous devons reconnaître que cet officium medium ne peut être assimilé à la notion de devoir telle que nous la connaissons dans la philosophie moderne, nous pouvons cependant nous demander si l'officium perfectum dont parle ici Cicéron n'est pas identifiable à l'idée d'une obligation idéale.

Certes, le katorthôma n'est pas assimilable à l'idée d'un devoir formel, mais à l'idée d'une perfection. C'est l'action qui correspond totalement avec le bien et le beau, celle qui exprime totalement le honestum. Or, selon les principes de la philosophie stoïcienne, il n'est pas possible de distinguer des degrés dans le bien, du moins pas dans les catégories du honestum ou de la sagesse complète. Le bien "est" ou il n'est pas, mais il n'y a pas d'action qui puisse, dans la perspective de la perfection, être considérée

\footnotetext{
13 Ethique de Nicomaque, Livre premier, II, 2

14 Jacques Maritain, La philosophie morale, p. 55

15 Cicero, De Officiis, I, 8
} 
comme «plus ou moins» parfaite. Dès lors l'officium perfectum, que nous pourrions appeler le «devoir pur», apparaît comme impossible à l'homme.

Cicéron a donc raison de parler des Paradoxes des Stoïciens, selon le titre d'un de ses ouvrages, car la morale parfaite qu'ils proposent est si idéale et si parfaite qu'elle en devient surhumaine. Le paradoxe est justement de décrire d'un côté une moralité impossible, et de conseiller, parallèlement, une moralité relative, à portée de l'humain. Epictète ironisait sur ce sage stoïcien qui n'avait jamais existé. «Je vois bien des hommes qui débitent les maximes des stoïciens, mais je ne vois point de stoïcien. Montrez-moi donc un stoïcien, je n'en demande qu'un. Un stoïcien, c'est-à-dire un homme qui, dans la maladie se trouve heureux, dans le danger se trouve heureux, qui mourant se trouve heureux, qui méprisé et calomnié se trouve heureux! Si vous ne pouvez me montrer ce stoïcien parfait et achevé, au moins montrez m'en un qui commence à l'être. Ne frustrez point un vieillard comme moi de ce grand spectacle, dont je l'avoue je n'ai pas encore pu jouir» ${ }^{16}$.

La morale stoïcienne ne peut donc échapper à la solution que lui propose Cicéron, à savoir de considérer l'officium medium - ou l'officium tout court, car Cicéron souligne qu'il ne retiendra que celui-ci dans sa discussion comme le rapport «convenable» entre les conditions concrètes de l'action et l'idéal qu' incarne l'idée de l'honestum. Maurice Testard le souligne dans l'introduction à sa traduction du De officiis: "L'officium, le devoir, .. intervient dans la doctrine de Cicéron, comme l'application, aussi fidèle que possible, du rapport idéal que représente l'honestum, aux cas concrets que pose la vie quotidienne; et il laisse place ainsi, dans la morale stoïcienne, à la notion de progrès moral» ${ }^{17}$.

Il y aurait donc bien un aspect formel de l'officium, dans son rapport à l'idéal moral, et en ce sens les officia peuvent très bien être considérés comme des devoirs; toutefois ces devoirs ne sont pas «le devoir», car ils dépendent tous des contenus particuliers de l'action, et la convenance qu'ils exigent n'est pas seulement celle qui les rattache à l'idéal, mais également celle qui les lie aux données concrètes de la situation. C'est pourquoi, comme tous les anciens, Cicéron ne peut séparer son analyse des devoirs de celle de l'utile. Toutefois, Cicéron échappe au relativisme qui lui viendrait d'une détermination morale par le seul utile, en rattachant sa doctrine à deux principes du stoïcisme, à savoir: d'une part la référence permanente de l'action à la nature ou à l'ordre des choses, par laquelle l'action est rattachée à l'universel et en particulier à une nature humaine fondamentale; et d'autre part à l'identification de la beauté morale et de son utilité, en faisant de la vertu elle-même le souverain bien, le bonheur ne se découvrant pas en dehors de la vertu elle-même. Ainsil'obligation, même relative de l'officium

16 Epictète, Entretiens, II, 49, cité in Jean Brun, Le stoïcisme, p. 114 -115

17 Maurice Testard, «Introduction» à la traduction de De Officiis, p. 57 - 58 
n'échappe pas à la finalité qui consiste à assurer le bonheur par la vertu. Mais ne revient-on pas ici à l'impasse qui consiste à faire de la fin un moyen?

Si le stoïcisme peut être considéré comme une étape importante dans le développement de la morale antique, en particulier dans cette identification du bonheur et de la vertu, il représente concrètement un des points de passage où la morale antique et la morale chrétienne se rencontrent. En effet, au delà de la vertu elle-même, la morale stoïcienne entrevoit une liberté intérieure totale, une liberté de l'âme que les vicissitudes de la vie ne peuvent pas atteindre. Le bonheur stoïcien n'est pour ainsi dire «plus de ce monde», La vertu-bonheur proposée par la morale du Portique se retrouve dans la vertu-bonheur que prêche le Sermon sur la Montagne, car puisque le bonheur n'est pas nécessairement là où on le cherche communément, les heureux sont peut-être ceux qu'on appelle malheureux, et inversément.

Stoïciens et Chrétiens se retrouvent dès lors pour affirmer que l'âme est dotée d'une dignité qui dépasse totalement les conditions concrètes de la vie humaine. Mais à l'inverse des stö̈ciens, cette morale n'apparaît pas aux chrétiens comme impossible, car ils voient dans la personne de Jésus le sage suprême qu'Epictète se plaignait de ne pas avoir rencontré parmi les philosophes. Si les Stoïciens se voyaient contraints à ne proposer que des devoirs relatifs, adaptés à une vie dans laquelle la sagesse totale ne pouvait s'épanouir, les chrétiens découvrent dans la personne de Jésus le témoignage vivant d'une sagesse éternelle, existante et possible, qui donne à l'obligation de la vertu une nouvelle dimension. Parl'intervention de Dieu dans l'histoire, la perfection idéale des stoïciens était mise à la portée des hommes. Le devoir allait ainsi s'enraciner définitivement dans l'absolu.

\section{Le paradoxe de l'obligation chrétienne}

De façon générale, la morale antique postérieure à Socrate n'a pas respecté la distinction que le sage d'Athènes avait introduite entre les choses divines et les choses humaines. Toutes les morales de l'antiquité, en effet, trouvent le fondement de leurs principes dans une connaissance de la nature et de l'être, et dans le respect d'une finalité inscrite dans la nature de l'homme. Même la liberté intérieure du sage stoïcien, qui dans une certaine mesure transcende la nature et les nécessités de la vie, demeure enfermé dans l'ontologie puisque la sagesse qu'elle vise n'est pas autre chose que la conformité de l'action humaine avec la nature. Les anciens étaient fort 
attachés à la croyance que toute réalité, humaine ou divine, était soumise à la même rationalité de l'être, ce qui permettait à l'homme non seulement de connaître le domaine réservé aux dieux, mais d'aborder la connaissance de Dieu lui-même. Cet attachement à la rationalité essentielle de tout être est certainement l'apport marquant de l'hellénisme à notre culture.

C'est au sein d'un tel monde que pénètre le message chrétien. Soulignons tout d'abord que le message de l'Evangile ne se présente pas comme une nouvelle philosophie systématique; ce n'est ni une cosmologie, ni une physique, au sens grec, apportant des révélations spéciales sur la nature du monde, ni une métaphysique mettant en lumière des aspects cachés de l'être. Le message chrétien se présente d'abord comme une morale, mais à la différence de toutes celles qui ont été construites dans le monde antique à partir de la rationalité, celle-ci s'appuie sur une relation directe avec Dieu. $C^{\prime}$ 'est donc un renversement complet de l'orientation socratique. Tandis que cette dernière se voulait presque exclusivement humaine, la morale chrétienne implique directement le divin. La perfection qu'elle propose n'est pas celle d'une humanité tendant vers son propre bonheur par le truchement de la vertu, c'est la perfection de Dieu même: «Soyez parfaits comme votre Père céleste est parfait» ${ }^{18}$. La morale chrétienne s'enracine définitivement dans un contact étroit avec le monde divin.

En ce sens, il n'est pas possible de considérer la morale chrétienne comme une morale philosophique. Mais elle a été tout de suite entraînée à s'expliquer et se justifier, dans le monde rationaliste hellénique, avec des arguments de nature philosophique. Ainsi, si l'on peut parler à juste titre d'une christianisation de la philosophie, il est tout aussi légitime de considérer la rencontre de la philosophie et du christianisme comme une véritable hellénisation de la révélation chrétienne.

Accomplie principalement à l'époque des Pères de l'Eglise, grecs et latins, cette hellénisation est déjà en germe dans la prédication de Paul aux Athéniens. En tentant de montrer aux anciens que le Dieu révélé est ce Dieu inconnu qu'ils révéraient par un autel spécial, Paul souligne que c'est celui «en qui nous avons la vie, le mouvement et l'être. C'est ce qu' ont dit certains de vos poètes: "Nous sommes aussi de sa race..." "19. Si le Dieu qui est venu à Jérusalem prêcher une morale d'amour est aussi celui en qui l'homme trouve la raison «du mouvement et de l'être», c'est-à-dire qui peut répondre définitivement aux questions de la métaphysique, alors le Dieu «d'Abraham, d'Isaac et de Jacob» est aussi celui des «philosophes et des savants». La même rationalité grecque s'applique aux deux mondes qui se rencontrent, et qui donnera son expression la plus accomplie dans la rationalité de la théologie chrétienne du moyen-âge.

18 Matthieu, 5; 48

19 Actes, 17; $27-28$ 
Toutefois, d'autres porteurs du message chrétien s'opposeront dès les premiers siècles àcette fusion du message religieux et de la pensée philosophique. Le credo quia absurdum de Tertullien, par exemple, se présente comme une des expressions les plus vives d'une telle opposition. On retrouvera cette attitude chez Pascal pour qui, justement, le Dieu «d'Abraham, d'Isaac et de Jacob» ne peut pas être celui des "philosophes et des savants». Cette tradition d'un christianisme non hellénisé, ou rationalisé, est à rapprocher de la distinction socratique entre choses humaines et choses divines. Si, pour Socrate, le domaine moral était réservé à la raison humaine, et la compréhension du monde à celui de la connaissance des Dieux, le christianisme se présentait au contraire comme un message moral religieux directement inspiré par un Dieu qui n'exigeait pas que l'homme comprenât les structures métaphysiques du monde pour savoir comment s'y conduire selon sa volonté. Seul le devoir que nous avons envers la divinité suffit à fonder la morale. Ainsi le domaine des choses divines devient celui de l'action humaine, du comportement de l'homme en relation avec Dieu, tandis que le domaine de la connaissance du monde, des choses et la nature est abandonné au pouvoir de la raison, à celui de la philosophie et de la science.

Il y aura donc deux éthiques chrétiennes. L'une se fondant sur la fusion de la morale évangélique et de la rationalité philosophique, c'est-à-dire sur une volonté divine qui s'exprime dans une nature qui fonctionne selon ses lois, et une autre éthique se fondant sur une relation directe entre l'homme et Dieu, échappant aux conditions naturelles de l'action, dans une relation d'amour de personne à personne entre la créature et son Créateur.

De ces deux éthiques issues du même message et de la même révélation, l'une se présente comme essentiellement philosophique et rationalisée, l'autre de caractère strictement religieux et, en un certain sens, mystique et irrationnel. La première s'exprime dans un langage hérité de l'antiquité, la seconde invente son expression propre dans une inspiration nourrie des relations personnelles de ses héros avec Dieu. C'est la rationalité qui fonde le devoir dans la première, tandis que la deuxième trouve la source de l'obligation dans la présence personnelle de Dieu dans la conscience morale des individus.

\section{Les nouvelles notions apportées par la révélation chrétienne à la philosophie morale}

Il n'est donc pas possible de parler d'une obligation chrétienne de façon univoque. La notion de devoir qui se développera dans l'histoire à partir de ces deux traditions éthiques deviendra en effet paradoxale, en raison même de sa double origine. Le problème qui a soulevé beaucoup de débats dans l'histoire de la pensée est de savoir si le devoir chrétien, tel qu'il peut être analysé philosophiquement, est d'origine exclusivement philosophique ou 
s'il est directement influencé par la dimension religeuse. Dans le premier cas, le devoir serait fondé sur une conception de la nature que la philosophie chrétienne aurait développée au-delà du point d'aboutissement de la philosophie ancienne; dans le deuxième cas, le devoir n'aurait d'autre source que la présence d'un absolu divin dans la conception de l'action morale, sans rapport avec la rationalité.

Les défenseurs d'une rationalité purement humaine n'ont pas manqué de mettre en cause les fondements philosophiques d'une notion qui, ne se retrouvant pas dans la philosophie antique, ne pouvait avoir comme origine que l'apport «irrationnel» de la révélation religieuse ${ }^{20}$. Mais les philosophes chrétiens ont tenté de répondre, dans le but de réconcilier la rationalité et la révélation pour expliquer la transcendance, que si nous acceptions l'idée d'un Dieu créateur, cause totale et consciente de la nature et de l'homme, la dépendance de notre être et de la rationalité du monde par rapport à sa volonté faisait qu'il était possible de construire une morale philosophique basée sur l'idée de devoir. Comme le dit Etienne Gilson, «le devoir ne se déduit pas d'une révélation, mais d'une doctrine de la création qui se déduit elle-même d'une métaphysique de l'être. Cette métaphysique ne se déduit de rien, que des exigences rationnelles de la pensée. Il est donc tout naturel qu'une morale de l'obligation ne se trouve jamais en contradiction avec la morale des Grecs: ils l'auraient découverte s'ils avaient approfondi leur métaphysique» ${ }^{21}$.

Un tel débat semble moins urgent en cette fin du XXe siècle, pourtant cette discussion nous apparaît utile, en particulier pour mettre en lumière certaines notions qui sont aujourd' hui présentes dans la réflexion philosophique sur la morale, que les Grecs ont peut-être entrevues mais qu'ils n'ont pas suffisamment élaborées, à savoir la «conscience», la «responsabilité personnelle», le «péché», et le «devoir envers autrui». Ce n'est qu'au moment où ces notions seront développées philosophiquement - leur origine purement morale et non métaphysique ne les empêchant pas d'être rationnelles - que pourra naître une philosophie morale de l'obligation.

L'idée de conscience, tout d'abord, qui ne se retrouve pas chez les anciens telle que nous la connaissons aujourd'hui, ne peut naitre qu'à travers l'idée d'un engagement de l'homme envers quelqu'un, envers autrui, - dans la perspective de quelqu' un qui attend quelque chose de moi, quelque chose que je lui dois - et finalement envers Dieu «qui m'appelle par mon nom». Cette notion a de plus été transformée dans le sens d'une morale transcendante

20 Voir, par exemple, un débat qui a eu lieu au début du XXe siècle dans la Revue philosophique en France, entre Victor Brochard et le Père Sertillange, janvier et mars 1901 .

21 Etienne Gilson, sIntention, conscience et obligation, in L'Esprit de la philosophie médiévale, p.343 
ou absolue, par la croyance en un bien moral qui subsistait en lui-même, dans l'être même de Dieu, et avec qui l'homme était appelé à entretenir une relation personnelle.

L'idée de responsabilité personnelle ne peut être dégagée d'une philosophie morale qui considère la perfection comme une simple conformité à la nature, une nature dans laquelle l'homme se trouve plongé. Si l'homme est une partie de cette nature à laquelle il doit se conformer, la conduite convenable ne dépend pas réellement de lui-même, mais de l'activité générale de la nature, - nous dirons aujourd'hui de ses impulsions - dont il n'est qu'une partie relativement peu indépendante.

L'idée de péché, ou de faute morale, n'apparaît chez les anciens, nous l'avons vu avec Socrate, que comme le résultat de l'ignorance. Le péché n'est donc pas lié à l'acte lui-même, mais à un manque de connaissance, et l'homme n'en est donc pas totalement responsable. Au contraire, la Genèse nous montre que la faute est devenue possible, pour Adam et Eve, au moment même où ils ont été capables de connaître le bien et le mal, après avoir mangé du fruit défendu. La connaissance ne permet donc pas d'éviter la faute, elle en est au contraire la condition indispensable. Le péché devient donc acte conscient et volontaire, engageant la responsabilité de l'individu.

Enfin l'idée d'un devoir envers autrui n'est possible que dans la perspective d'une loi suprême d'amour qui fonde toute action morale. Celle-ci ne peut donc plus se résumer à poursuivre un bonheur subjectif, qui devient d'ailleurs très relatif, comme le suggère ouvertement le Sermon sur la Montagne, quand il annonce le bonheur des malheureux! Le message chrétien propose au contraire une morale qui s'épanouit dans une ouverture vers autrui; vers Dieu d'abord, vers le prochain ensuite. L'homme n'est donc plus appelé à suivre une morale déterminée par ce qu'il est lui même et par ce qu'il désire naturellement, mais à travers ce que l'autre, Dieu ou le prochain, attend de lui.

Ce n'est que plus tard, dans le déroulement de l'histoire de la pensée, que ces notions seront dégagées pour elles-mêmes, dans l'élaboration d'une philosophie essentiellement morale. En effet, sans les notions de péché, et plus tard de responsabilité et de conscience (que nous retrouverons chez Rousseau et chez Kant) il était plus difficile pour les Grecs de séparer la morale de la métaphysique. Mais bien qu'elles furent ignorées des Grecs, et qu'elles furent élaborées ou renouvelées après eux, ces notions ne sont pas nécessairement irrationnelles. Si elles le sont aux yeux de certains, c'est peut-être par manque d'ouverture à l'histoire même de la philosophie.

\section{Intériorisation et intention}

La morale juive avait mis l'accent principalement sur la loi, Loi divine et absolue, dictée aux hommes sur le Sinaï. L'observation de la loi était toute 
la morale, et toute justification religieuse. Le Nouveau Testament n'abolira pas la loi, mais en la dépassant, il visera à ancrer la morale nouvelle à un niveau supérieur. L'amour ne se fonde pas sur la loi; c'est elle qui trouve en lui sa signification. A elle seule, la loi devient inutile. "Toute la loi se résume en un précepte: tu aimeras ton prochain comme toi-même» ${ }^{22}$.

Mais en déplaçant l'essence de la morale de la loi à la charité, le christianisme ne transforme pas seulement le contenu de la morale, il lui donne une nouvelle orientation et un nouvel enracinement dans la conscience. L'essentiel du caractère moral n'est plus à trouver dans le but à poursuivre, mais dans l'intention de l'être agissant. Il faut aimer Dieu et son prochain «de tout son coeur, de toute son âme et de tout son esprit». C'est donc en lui-même que le sujet agissant devra rechercher la bonté de son action. L'homme est moral s'il agit «par» amour, et non pas s'il fait ceci ou cela selon des préceptes qui lui sont extérieurs. C'est finalement dans l'intention de l'homme que se trouve le vrai caractère moral de son action. $\mathrm{La}$ conséquence en sera une intériorisation de la moralité.

Préparées par la tradition juive qui parle d'un Dieu connaissant «les reins et les coeurs", et qui juge l'homme dans son intention et sa foi, à l'instar du prophète qui demande à Dieu de ne pas être confondu avec «ceux qui disent à leur prochain des paroles de paix, mais dont le coeur est plein de méchanceté» ${ }^{23}$, les exhortations de l'Evangile insistent sans cesse sur le rôle primordial de l'intention morale qui se trouve dans le coeur de l'homme, qui précède l'action et détermine sa valeur morale. «Ce qui sort de la bouche vient du coeur, et c'est cela qui souille l'homme. Car c'est du coeur que viennent les pensées mauvaises, ... mais manger sans se laver les mains, cela ne souille point l'homme» ${ }^{24}$. La valeur morale de l'action est donc déterminée antérieurement à l'action, et surtout à la loi par rapport à laquelle l'action poura être jugée. Il y a donc une réalité essentiellement morale, invisible du dehors et de ce fait échappant à la loi, connue seulement de Dieu et de la conscience du sujet, qui précède l'action, qui la vise et qui lui donne sa valeur. Le péché ou le mérite se trouveront dans la pensée bien avant l'action. C'est là qu'il faut en chercher l'origine, et cette origine devient dès lors plus importante dans le jugement moral que le mérite ou le péché luimême. «A partir de ce moment,» écrit Etienne Gilson, «on peut dire que l'essence du bien et du mal va se trouver transférée de l'acte à la volonté»" ${ }^{25}$.

La morale chrétienne trouvera dès lors son fondement dans la volonté et la pureté du coeur, ce qui ouvre la voie plus tardive vers le kantisme. Une telle orientation rejoindra par d'autres voies la notion stoïcienne du bonheur.

23 Galates, $5 ; 14$

Psaumes, 27; 3

24 Matthieu, 15; 18-20

25 Etienne Gilson, op. cit., p. 327 
En convertissant la valeur morale à l'intériorité de l'agent, le christianisme favorisera une intériorisation du bonheur lui-même. Celui-ci ne sera plus considéré comme le but ou le résultat d'une action, mais il se confondra dorénavant avec cette nature du coeur, fondement de la moralité. C'est en ce sens qu'on peut interpréter les béatitudes du Sermon sur la Montagne. «Heureux ceux qui ont le coeur pur, ... car leur action obtiendra la justice $\mathrm{du}$ Seigneur» ${ }^{26}$; Etre heureux ou vouloir le bien, finalement, seront une seule et même chose, une identique orientation préalable de la volonté. Or cette orientation préalable, souligne Gilson, «lorsqu'on lui donne un nom philosophique, puisqu'elle désigne le mouvement réel de la volonté tendant vers sa fin, on la nomme tout simplement l'intention ${ }^{27}$.

La perspective chrétienne conduit donc à construire une morale de l'intention dans laquelle la conscience du sujet jouera un rôle essentiel. C'est sur cette conscience en effet que s'établira finalement la moralité. En s'engageant sur cette voie, toutefois, la philosophie morale chrétienne préparait le chemin au «subjectivisme» progressif de la moralité. La notion d'obligation morale permettra de compenser cette tendance. Cette notion n'est donc pas née directement de la morale chrétienne, mais indirectement, pour corriger pour ainsi dire, l'intériorisation totale de la morale à laquelle l'éthique chrétienne pouvait conduire.

Il n'est pas sans intérêt de constater que ce recours à l'obligation dans la philosophie chrétienne, indispensable pour échapper au danger de subjectivisme auquel elle conduisait, rejoint la thèse principale de ce livre. Quand la liberté d'action devient sans limite, c'est le sujet lui-même qui doit devenir maître de ses actes. L'obligation est aussi nécessaire à l'agent moral chrétien trouvant «en son coeur» la justification morale de son action, qu'elle est indispensable à l'agent économique dans un marché totalement dérégularisé. C'est quand tout est possible que l'homme devient responsable de tout et doit trouver en lui-même le pouvoir de s'auto-contrôler. L'obligation est née au cours du cheminement de la pensée qui progressait vers une affirmation plus complète de la liberté. L'obligation, nous le verrons, ne limite pas la liberté, elle en est la manifestation.

\section{Conscience et rationalisation}

La notion de volonté prend donc une place centrale dans la construction de l'éthique chrétienne. Cette volonté, pourtant, et en cela la philosophie chrétienne suit presque à la lettre la philosophie antique, n'est jamais assimilée à une spontanéité aveugle, c'est-à-dire une forme d'impulsion

26 Matthieu, 5; 9-10

27 Etienne Gilson, idem 
centrifuge qui n'aurait pas de finalité précise. La volonté ne peut tendre vers une fin quelconque sans la connaître. Si la connaissance des fins est indispensable à la volonté, la raison doit donc obligatoirement intervenir dans l'action.

C'est donc la raison qui doit définir les fins que poursuit la volonté. Mais cette raison n'est pas infaillible, elle est la raison d'une nature déchue - la tradition religieuse intervient ici, à nouveau, dans la construction de l'éthique chrétienne - et peut donc se tromper. C'est pourquoi, la conscience, aussi sincère soit-elle, peut être dans l'erreur. Car la volonté n'agit pas en fonction de la fin telle qu'elle est en elle-même, mais en fonction de la fin telle qu'elle est perçue par la raison et représentée par elle à la volonté. L'objet est, aux yeux de la volonté, indifférent en lui-même; il ne devient bon ou mauvais que par l'intervention de la raison, selon qu'elle le présente comme un bien à faire ou un mal à éviter. Conscience rationnelle et volonté doivent donc s'intégrer dans un seul acte d'intention pour fonder la valeur morale d'une action. En dehors de cette conscience et de cette intention il n'y a pas de morale possible. La formule de Thomas d'Aquin est claire: «omnis voluntas discordans a ratione, sive recta sive errante, semper est malan ${ }^{28}$.

Pour qu'une action morale soit bonne, dès lors, il faut que l'homme agisse dans la conviction profonde que le choix qu'il fait par sa propre raison est bon, même si celui-ci est peut-être objectivement erroné. En d'autres termes, il ne peut y avoir de moralité sans autonomie morale du sujet, c'està-dire sans un pouvoir de l'homme à se déterminer lui-même dans son action. Saint Paul le disait à sa façon: «tout ce qui ne procède pas d'une conviction est péché» ${ }^{29}$.

La valeur morale de l'action doit donc venir d'une inspiration profonde de la conscience, une sorte de foi dans la bonté de la fin visée. Cela signifie, finalement, que l'homme ne peut pécher que s'il est pleinement conscient du mal qu'il va faire ${ }^{30}$. La doctrine morale chrétienne se sépare en ce sens de la morale socratique pour qui le pécheur (bien que la notion de péché n'existât pas en ce temps) n'était qu'un ignorant du bien et de la vertu. Au contraire, pécher, pour la philosophie chrétienne, c'est agir contre sa conscience. Ne pas savoir n'est pas être mauvais; c'est être simplement prisonnier de l'amoralité que donne l'innocence. En d'autres mots, il n'y a pas de moralité sans affirmation du sujet en tant que responsable de son acte.

En intériorisant la valeur morale, l'éthique chrétienne conduit ainsi à affirmer la place centrale de la conscience individuelle dans la décision

28 Thomas d'Aquin, Somme théologique, Ia, Ilae, q. 19, art. 5

29 Romains, 14; 23

30 On retrouve ce thème chez Pascal, Quatrième Provinciale 
morale. La notion de conscience est considérée ici dans le double sens de conscience de soi et de conscience morale. Nous y reviendrons.

Mais cela demande aussi de ne pas faire une confiance aveugle à la raison humaine qui, déchue depuis le péché originel, n'est pas infaillible. C'est pourquoi, en raison de l'imperfection fondamentale de l'homme, il sera nécessaire, Thomas d'Aquin y insiste, de compenser les faiblesse de la raison humaine par un recours quasi permanent à la loi éternelle de la raison divine $^{31}$. Ce recours sera, pour ainsi dire, une façon de garantir la bonté de nos buts et la valeur morale de nos actions en évitant de leurrer notre volonté par une raison défaillante. C'est une façon de fixer l'intention vers le bien objectif que ne peut manquer de connaître la raison divine.

Mais cette nécessité de recourir à la loi divine est avant tout justifiée par la nature faillible de la raison humaine. Une philosophie qui n'adhèrerait pas à l'idée d'une nature déchue comme point de départ de la réflexion morale, pourrait se débarasser de ce recours et faire de la raison, acceptée comme telle avec son pouvoir propre et son universalité, l'unique moyen de protéger l'intention individuelle contre la tentation de la subjectivité absolue. Nous devrons nous demander si le recours kantien à la forme, et par cette forme à l'universalité de la raison, ne joue pas le même rôle que le recours thomiste à la raison divine.

Si la raison toute puissante de Dieu est seule capable de déterminer infailliblement les buts de l'action morale, il n'empêche que cette action ne peut être entièrement morale si l'agent l'entreprend par le truchement de sa propre conscience, en d'autres mots, s'il la choisit librement. La loi divine, en effet, aussi infaillible qu'elle soit, ne peut déterminer la volonté à agir sans la participation de la conscience autonome du sujet. Luther a fortement insisté sur ce point. C'est pourquoi l'observation de la loi sans la conviction intérieure ne suffit pas à la moralité. Dès lors, ni la raison divine, ni l'autonomie du sujet, prises séparément, ne suffisent à fonder la valeur morale de l'action. Il faut qu'elles se rencontrent dans une même intention.

\section{L'obligation morale}

C'est dans le but de garantir cette rencontre de la rationalité divine et de la libre conscience de l'homme qu'intervient la notion d'obligation morale. Comment, en effet, une telle rencontre peut-elle être possible si ce n'est dans l'affirmation du caractère absolu de la raison et de la loi divines, qui s'imposent à l'homme et l'obligent au nom même de cet absolu. L'idée de création est ici essentielle. La loi divine peut orienter l'intention de

31 "ubi deficit humana ratio, opportet ad rationem aeternam recurrere», Somme théologique, la, IIae, q. 20, art. 4 
l'homme parce qu'elle s'impose avec l'autorité de la puissance créatrice dont dépend non seulement l'être de l'homme, mais aussi sa raison. La conscience humaine n'a donc aucune difficulté à reconnaître comme un devoir la voie que lui propose son Créateur.

Car la loi morale proposée par la raison divine ne se présente pas comme une détermination, mais comme un devoir. L'intériorisation de la moralité et la révélation de la conscience morale ont en effet appelé l'homme à l'autonomie, c'est-à-dire à la capacité de déterminer lui-même sa propre action. La perspective chrétienne a ainsi permis de développer la notion de liberté; voilà pourquoi la relation entre le sujet moral et son Créateur est une relation de devoir, et non pas de dépendance. Nous sommes donc loin d'un approfondissement de la métaphysique des anciens, et Gilson a tort de croire que c'est la voie qu'ils auraient dû suivre pour parvenir à la notion d'obligation. Il n'y a obligation dans le rapport de l'homme avec Dieu, que parce qu'il y a une reconnaissance de la conscience et de l'autonomie morale. Le devoir est finalement le rapport d'une raison limitée par la nature déchue avec le caractère absolu de la raison divine, sans nier notre liberté. Le recours à l'obligation, dans la philosophie morale d'inspiration chrétienne se trouve dans la reconnaissance des limites de la raison humaine.

\section{Obligation et morale d'amour}

Mais la philosophie chrétienne ne prétend pas contenir tout le message de l'Evangile. La dimension plus spécifiquement religieuse de ce dernier n'a pas manqué d'influencer, de son côté, les attitudes morales, etl'obligation n'y trouve pas nécessairement sa place. Le message du christianisme n'a pas été entièrement soumis à la rationalisation hellénique. L'Evangile, en effet, propose un enseignement moral direct et concret dans lequel l'obligation des philosophes ne trouve pas sa raison d'être. Le message d'amour ne parle pas de loi, mais de rapports entre personnes. Ce n'est pas une relation entre une conscience et une loi mais entre une personne et une autre, liées par un amour réciproque. Amour de Dieu d'abord, amour d'autrui, des proches mais aussi de l'inconnu et de l'ennemi, du pauvre et du malade. Cet amour demande à l'homme de se convertir intérieurement avant même de se demander ce qu'il doit faire. La charité est une attitude qui remplace toutes les indications de la loi et toutes les obligations.

C'est pourquoi il est paradoxal de parler d'obligation chrétienne. Etranger à l'idée d'une obligation morale fondée sur l'absolu de la loi divine, le message moral du Christ insiste continuellement sur la nécessité d'un amour qui, par sa nature, rejette toute référence à une loi ou à un devoir. Ce qui a conduit Saint Augustin à résumer cet Evangile par la formule: ama et fac quos vis! S'il est donc vrai que la pensée chrétienne rationalisée est à l'origine de la notion d'obligation morale, il est tout aussi certain que la 
morale évangélique peut être source d'une conception de l'action humaine qui supprime toute référence à l'obligation. Seul reste l'amour pour autrui qui empêche que le "fais ce que tu veux" n'aboutisse pas au relativisme moral, à l'arbitraire et à l'individualisme. Pour être morale la conscience ne doit pas se soumettre à une obligation, elle doit simplement prendre la place de l'autre.

Il serait donc faux, ou du moins très paradoxal, de présenter l'obligation morale comme tout l'héritage moral du christianisme dans notre histoire. Certes, l'obligation est historiquement d'origine chrétienne, et il est important de le souligner, mais l'obligation a enrichi notre héritage philosophique principalement à travers l'apport supplémentaire que la pensée rationalisante a ajouté au message exclusivement religieux et moral de l'Evangile. En ce sens précis, il est possible de dire que l'obligation morale n'est pas réellement et authentiquement chrétienne.

\section{L'absolu du devoir kantien}

La philosophie morale de Kant se situe dans le développement historique des doctrines éthiques comme une rupture, un renversement de perspective. On l'a justement appelée une «révolution copernicienne». Mais elle se situe cependant dans une continuité qui la rattache aux philosophies morales précédentes sur lesquelles elle s'appuie: philosophie antique, rationalisation du message religieux de l'Evangile, intériorisation progressive de la conscience et de la valeur morales. Il faut donc voir dans la pensée kantienne autant un aboutissement qu'un point de départ entièrement neuf.

Parmi les influences directes, il est important de mentionner, en dehors du rationalisme du XVIIle siècle, l'optimisme rationaliste de Voltaire, la pensée systématique d'un Wolff' et l'impact plus direct du piétisme qui a marqué Kant dès son plus jeune âge. Ce piétisme explique en partie le caractère religieux qui pénètre la pensée morale kantienne. Le piétisme insistait en effet sur le rôle essentiel de la pureté du coeur et de l'intention, en se méfiant très fortement des attraits sensibles qui risquaient, par une

32 Christian Wolff (1679 - 1754) a construit une oeuvre «systématique» considérable dans un rationalisme typique du XVIIIe siècle, qui représente une synthèse entre la scolastique tardive et des penseurs comme Leibniz et Locke. Il a eu une grande influence sur son temps. Son oeuvre principale est le Jus Naturae (9 volumes) qui constitue une partie de sa philosophie pratique. 
sorte de mysticisme désordonné, de dégrader la religion elle-même. Le piétisme apparaît beaucoup plus comme une religion s'appuyant sur la morale, que comme une morale construite à partir de fondements religieux.

Il faut mentionner, d'autre part, le contact que Kant a poursuivi avec les écoles anglaise et écossaise, en particulier avec des penseurs comme Shaftestbury, Hutcheson, Hume, qui lui ouvrent des horizons nouveaux. C'est à leur contact qu'il acquiert la conviction que l'homme est doté d'un penchant naturel à la vertu, qu'il faut dès lors s'appuyer sur le sens commun, et tenir compte de la capacité de l'homme à reconnaître spontanément ce qui est moral dans toute tentative de construction d'un système éthique.

C'est enfin à Rousseau que Kant doit ce qu'il appelle lui-même une «révélation». Rousseau, écrit Victor Delbos qui a analysé les sources de la pensée morale kantienne, «achève de pousser Kant hors des voies du rationalisme wolffien, et il se rencontre avec les philosophes anglais pour le porter à voir dans le sentiment l'origine de la moralité ${ }^{33}$.

Mais tandis que le sentiment demeurait, chez Rousseau, très proche de l'émotif, du sensible, du domaine de l'empirique, Kant va tenter de montrer que cette conscience du bien et du mal, que Rousseau appelait encore «un instinct divin de la norme», est essentiellement de caratère rationnel, qu'il n'est pas autre chose que la raison elle-même considérée comme pratique. Sa réflexion le pousse donc à rechercher le principe de cette raison pratique, qui est en l'homme et lui permet d'éviter les erreurs. Le but de sa tentative est de proposer une formule qui permette à l'homme de connaître de façon tout à fait exacte ce qu'il y a à faire pour résoudre les problèmes pratiques.

En plaçant la valeur morale de l'acte en l'homme lui-même, dans la rationalité de sa conscience morale, Kant parachève, pour ainsi dire, le mouvement d'intériorisation progressive de la moralité qui est l'une des conséquences de l'hellénisation du message évangélique. Kant admet d'ailleurs cette filiation puisqu'il reconnaît dans la loi évangélique cette «loi de toutes les lois ... l'intention morale dans toute sa perfection» ${ }^{34}$. Mais au delà de l'élaboration d'une théorie éthique, Kant est conscient, à la suite de Rousseau, que l'affirmation de la rationalité de la morale conduit à une nouvelle conception de l'homme, qui lui impose de s'engager dans une perspective religieuse et d'affirmer «philosophiquement» la nécessité de postuler Dieu et l'immortalité de l'âme. C'est ainsi à une reconstruction de la philosophie de l'homme que conduit la philosophie morale kantienne.

33 Victor Delbos, La philosophie pratique de Kant, p. 126. Il est ici fait allusion au passage célèbre de la "Profession de foi du Vicaire savoyard", dans l'Emile ou de l'éducation (1762) dans lequel Rousseau affirme que la conscience est une forme «d'instinct divin de la norme" permettant de distinguer quasi spontanément ce qui est bien et ce qui est mal.

Kant, Critique de la raison pratique, p. 87-88 


\section{L'a priori et le formalisme}

L'affirmation de la suprématie de la conscience, chez Rousseau, ne représentait pas une rupture radicale avec la tradition de la philosophie chrétienne. Thomas d'Aquin et la philosophie scolastique avaient clairement montré que seule une action fondée sur la conscience pouvait prétendre au caratère moral. Agir contre elle, même si la raison trompait la volonté, était toujours mal.

Mais cette philosophie était fondée, souligne Kant, sur une conception particulière de la volonté, héritée de la morale antique, à savoir son hétéronomie essentielle. La volonté, en effet, n'agissait que sous la détermination d'un bien-objet extérieur à elle-même. Certes ce bien devait toujours luiêtre présenté par la raison comme bonum apprehensum, comme un bien jugé par la raison, mais la volonté continuait à dépendre d'une motivation qui lui demeurait extérieure. Le souci premier des philosophies construites sur ce modèle était donc de garantir la rectitude de la raison qui, dans la perspective chrétienne, était une raison faillible, d'où la nécessité de recourir à la raison divine pour compenser cette faiblesse. Or Kant ne voit pas la nécessité de remonter jusqu'à la raison divine, comme distincte de la raison humaine, pour trouver le critère infaillible de la moralité. Ce qui l'en empêche, c'est qu'il sait que l'entendement et l'intuition sensible sont limités, et qu'il n'y a dès lors pas de métaphysique possible comme connaissance. Il serait donc vain de fonder la moralité sur "un approfondissement de la métaphysique». La raison, comme l'a montré Kant dans la Critique de la raison pure, ne peut affirmer l'existence d'un objet en soi, et donc encore moins un objet absolu tel Dieu comme créateur du monde. Aussi, si la morale doit s'appuyer sur la réalité d'un absolu qui puisse la fonder sur un caractère obligatoire, ce n'est pas en dehors de la raison elle-même qu'il faut la chercher.

Avec Rousseau, la conscience devient un «juge infaillible du bien et du mal, qui rend l'homme semblable à Dieu». L'idée d'une nature originellement déchue disparaît. La pensée de Kant, en partant de l'affirmation du pouvoir absolu de la conscience, tente donc de démontrer les conditions de son infaillibilité. Certes, Kant était un esprit trop religieux pour oser imaginer que l'affirmation du pouvoir suprême de la conscience permît à l'homme de se passer de Dieu, mais il ne pouvait pas utiliser ce Dieu comme une finalité morale, car cela aurait confirmé l' hétéronomie de la volonté et aurait fait de la morale postérieure à l'Evangile une morale soumise aux mêmes principes que les morales de la nature proposées par les Grecs.

Partant de l'idée que, pour avoir une valeur morale, c'est-à-dire pour pouvoir fonder une obligation, une loi doit se présenter comme une absolue nécessité, et que les commandements qu'elle propose soient valables pour tous les êtres doués de raison, le principe sur lequel elle repose doit donc se trouver a priori dans les seuls concepts de la raison. Si l'homme, dès lors, prend conscience du problème moral, ce n'est pas en tant qu' homme plongé 
dans une nature ou placé dans des circonstances empiriques particulières, mais en tant qu'être doué de rationalité, soumis aux mêmes lois que tous les êtres raisonnables. Et c'est par là qu'il participe à la nature de Dieu puisque le principe de la moralitê, tel qu'il peut être tiré de cette rationalité, n'est pas «simplement limité aux hommes, mais il s'applique à tous les êtres finis qui ont raison et volonté; bien plus, il comprend même l'être infini, en tant que suprême intelligence ${ }^{35}$. Pour construire une telle morale, Kant se voit donc conduit à substituer «à la notion d'homme le concept d'être raisonnable»"

En conséquence, les principes de la morale aprioriste de Kant ne sont pas saisis par la raison, comme les objets d'une connaissance, mais issus de la raison, comme les exigences spécifiques de la rationalité. Pour construire cette morale, dès lors, il faut laisser la raison se révéler spontanément à elle-même, en dehors de toute référence empirique et en dehors de tout modèle. La bonté morale d'un acte, son caractère moral essentiel, ne pourra donc se situer dans l'acte lui-même, mais dans ce qui est préalable à l'acte et qui le produit.

Or, c'est de la volonté que viennent les actions. C'est elle qui nous fait agir. C'est donc dans la volonté elle-même que nous devons rechercher si nos actions sont moralement bonnes ou mauvaises, c'est en elle qu'il faut trouver ce qui conditionne a priori leur valeur morale. Or, dit Kant: «de tout ce qu'il est possible de concevoir dans le monde, et même en général hors du monde, il n'est rien qui puisse sans restriction être tenu pour bon, si ce n'est seulement une BONNE VOLONTE»" ${ }^{37}$. Toutes les autres qualités du caractère, de l'intelligence, du tempérament, de l'habitude, peuvent certes être considérées comme bonnes, mais elles ne le sont pas en elles-mêmes. «Ce qui fait que la bonne volonté est telle, ce ne sont pas ses oeuvres ou ses succès, ce n'est pas son aptitude à atteindre tel ou tel but proposé, c'est seulement le vouloir; c'est-à-dire que c'est en soi qu'elle est bonne» ${ }^{38}$.

C'est donc dans le sujet que se trouve la moralité et son principe, et cela implique l'affirmation de l'autonomie absolue de la volonté. C'est cette autonomie qui fonde la liberté. Pourtant, cette volonté risquerait d'apparaître comme une «transcendante chimère» si la raison ne pouvait s'en saisir. Comment la raison conduit-elle notre vie pratique? Si elle ne devait jouer aucun rôle, la nature, dit Kant, aurait mieux fait de soumettre notre activité entièrement aux instincts. Mais si l'homme est un être raisonnable, il faut supposer que cette raison joue un rôle particulier dans la direction de la volonté, c'est-à-dire qu'elle ne doit pas être seulement théorique, mais aussi pratique. Toutefois, puisque la volonté est autonome et qu'il existe une liberté humaine fondée sur cette autonomie, il faut que la vraie destination

\footnotetext{
35 Ibid., p. 32

36 Ferdinand Alquie, Introduction a la Critique de la raison pratique, p. xii

37 Kant, Fondements de la métaphysique des moeurs, p. 87

38 Ibid., p. 89
} 
de la raison "soit de produire une volonté bonne, non pas comme moyen en vue de quelque autre fin, mais bonne en soi-même» ${ }^{39}$. En d'autres termes, la raison doit déterminer la volonté à être bonne, et non pas à vouloir le bien. Ce qui fait que la raison pratique kantienne a pour but d'assurer la moralité de l'intention du sujet, et non pas la moralité des fins qu'il vise. Nous nous trouvons devant une morale entièrement intériorisée, dont le principe pourrait être, à la limite: «que ta volonté soit bonne, et fais ce que tu veux!».

Mais quels sont en fait les principes pratiques, et objectifs, c'est-à-dire valables pour tous les êtres raisonnables, que la raison peut proposer pour déterminer la volonté à être bonne? Ces principes ne peuvent, tout d'abord, être matériels, car ils s'adresseraient alors à la faculté empirique de désirer, et ne pourraient déterminer la volonté à être bonne en elle-même. C'est pourquoi ces principes doivent être "formels", car un être raisonnable ne peut pas se représenter ses propres maximes pratiques subjectives comme des lois universelles sans «admettre que la simple forme par laquelle ils s'adaptent à une législation universelle en fait par elle seule des lois pratiques $»^{40}$. La forme de la loi est donc l'unique détermination rationnelle possible que l'homme, en tant qu'être raisonnable doté d'une volonté autonome, peut se représenter pour sa volonté. C'est donc par la forme que la morale de l'intention évite de tomber dans le subjectivisme absolu.

Le premier principe, la condition suprême de toutes les maximes, est donc d'agir de telle façon que ce qui détermine la volonté puisse toujours être proposé comme principe d'une législation universelle. Ce principe n'est pas donné par une connaissance de quelque objet, mais issu de la raison elle-même. La conscience de cette loi fondamentale, que Kant appelle un fait de la raison, est une donnée originelle immédiate au delà de laquelle il n'est pas possible de remonter. Dès lors, conclut Kant: «la raison pure est pratique par elle seule et donne à l'homme une loi universelle, que nous nommons la loi morale ${ }^{41}$. Contrairement aux lois de la nature empirique, qui visent une fin matérielle, les lois de la moralité, directement issues de la raison en tant que pratique, sont nécessairement formelles et a priori. C'est pourquoi, même si elles déterminent la volonté, elles ne contredisent pas la liberté. Au contraire, ce sont ces lois qui permettent à l'homme de découvrir et de connaître sa liberté.

\section{La moralité par le devoir}

On constate certes que les attraits et les penchants sensibles peuvent parfois pousser la volonté à agir conformément à ce que demande la loi, mais

\footnotetext{
39 Ibid., p. 93

40 Critique de la raison pratique, p. 26

41 lbid., p. 31
} 
Kant dit de ces actions qu'elles ne sont que «légales» et non pas authentiquement «morales», car la raison n'a pas pour but de faire produire des actions bonnes, mais uniquement de transformer la volonté en volonté bonne. C'est pourquoi le seul mobile réellement moral, qui ne dépend d'aucun penchant sensible, est celui du respect pour la loi. Ce sentiment de respect est pour Kant l'authentique «sentiment moral», produit exclusivement par la raison. Le concept qui l'exprime est celui du devoir. C'est lui qui fonde finalement la moralité et se trouve à l'origine de la détermination "morale» de la volonté. L'idée de devoir ne demande pas seulement qu'il y ait, dans l'action, une conformité à la loi, mais que le respect pour la loi soit le seul mode de détermination de la volonté par cette loi. "C'est là-dessus que repose la différence entre la conscience d'avoir agi conformément au devoir et d'avoir agit par devoir, c'est-à-dire par respect pour la loi» ${ }^{42}$.

Le devoir kantien n'est pas un attribut secondaire ou une des caractéristiques acquises de la moralité, comme l'obligation chrétienne pouvait l'être dans le rapport de la raison humaine faillible à l'absolu divin; il doit au contraire en être le fondement, son principe premier, son critère formel et ultime. Issu des exigences aprioriques et formelles de la raison pratique, le devoir se présente comme absolu. Il jaillit, pour ainsi dire, de la nécessité de l'intention elle-même. C'est pourquoi le devoir ne peut pas être considéré comme une chose, une réalité ayant sa propre spécification ou essence; il n'est que la forme que doit revêtir la détermination de la volonté pour que celle-ci soit bonne et l'action authentiquement morale. Ce n'est pas non plus le principe d'une action concrète, celui=ci se trouvant dans lâ maaximê subjective. Le devoir est la forme que doit prendre cette maxime pour que cette dernière soit morale.

Pure forme, le devoir n'existe que par l'acte de la volonté auquel il donne une valeur morale, aussi le devoir n'existe pas comme une notion ayant la réalité des idées platoniciennes. Si la loi, elle, peut exister et être proposée antérieurement à l'action, le devoir n'est que la forme de l'action, et n'existe pas sans elle. C'est pourquoi il n'est pas possible d'agir pour le devoir, en faisant de celui-ci la fin de l'action. Le devoir n'est pas un bien ultime comme Dieu pouvait l'être dans la perspective chrétienne. Toute tentation de le faire enlèverait au devoir sa nature formelle, qui en donne justement son caractère absolu et universel. Mais s'il n'est pas envisageable de penser la réalité du devoir en dehors de la détermination de la volonté, il n'est par contre pas possible de considérer une action comme morale sans qu'elle soit «informée» du devoir. En ce sens, le devoir devient l'élément constitutif essentiel de la moralité et, à la limite, les notions de devoir et de moralité tendent pratiquement à se confondre. 
Il est maintenant facile de comprendre en quoi consiste cette «révolution copernicienne» de la pensée morale de Kant, en particulier en ce qui concerne la définition de ce qu'il faut faire pour que l'action soit morale. Il s'agissait chez les anciens de trouver ce qu'il convenait de faire pour atteindre une fin. L'obligation, si l'on peut parler en ces termes de l'exhortation qui se rattachait à cette loi individuelle, se rattachait de façon concrète à chaque cas particulier, comme dans les officia de Cicéron. Et dans chaque situation le bonheur subjectif de l'agent devait se réaliser. La loi morale était déterminée par le but à atteindre qui devait conduire au bonheur de l'homme. Mais aucune de ces lois ne se présentait comme absolue; il n'existait pas en fait de devoir, ou d'obligation, proprement dite. Dans la philosophie chrétienne, ce qu'il convient de faire prend une valeur absolue parce que cette "convenance» trouve sa raison ultime en Dieu, Créateur des moyens et lui-même fin dernière de l'action morale. Le refus du moyen, Ia loi, correspond dès lors à renoncer à la fin, puisque l'une et l'autre émanent de la même source créatrice. Certes la philosophie morale chrétienne ne renonce pas à l'idée d'un bonheur humain, fin ultime subjective de l'action morale, cependant le chrétien ne doit pas rechercher ce bonheur en premier lieu, mais la volonté du Créateur. Il doit aimer Dieu par dessus tout. La loi morale nous oblige parce qu'elle est ancrée dans l'absolu divin.

Pour Kant, au contraire, la loi morale oblige non pas parce qu'elle se trouverait rattachée à un absolu en dehors d'elle, mais parce qu'elle est la forme même de l'universel. Les kantiens diront que ce n'est pas parce que la loi est morale qu'elle oblige, mais au contraire qu'elle est morale parce qu'elle oblige. Le devoir devient donc le fondement ultime de la moralité. Tandis que le devoir chrétien était fondé dans la nature même de l'être moral, chez Kant c'est l'être moral qui est constitué en tant que moral par le devoir. La loi s'est substituée à l'être. Le devoir ne vient pas des exigences de la moralité, c'est la moralité qui est issue du devoir. C'est de lui que viennent à la fois la pureté morale absolue, puisqu'il ne fait appel à aucun penchant et à aucune faculté sensible du désir, et la dignité de l'homme, puisqu'il élève l'homme au dessus du monde sensible.

\section{Le sujet du devoir et la conscience morale}

Construite sans l'aide d'une connaissance préalable de la nature humaine ou d'une anthropologie empirique, la philosophie kantienne du devoir est amenée à proposer, en fait, une nouvelle «anthropologie», non pas empirique cette fois, mais morale. Il s'agit d'une affirmation de la dignité de l'homme comme sujet du devoir.

Puisque le devoir se présente à l'esprit comme absolu, il ne peut que forcer l'homme à renouveler sa pensée sur lui-même à partir de cette prise de conscience. C'est, dans une certaine mesure, un retour au «connais-toi toi-même» de Socrate, mais non plus situé cette fois devant l'impasse d'un 
savoir qui ne pouvait être pratique, mais face au savoir pratique suprême, la conscience du devoir. Il est donc légitime de parler du devoir dans les mêmes termes que Kant utilisait pour caractériser les «effets» de la loi morale: il n'est pas possible de savoir a priori "pourquoi la loi morale fournit en elle-même un mobile, mais ce que, en tant que mobile, elle produit (ou pour mieux dire, doit produire) dans l'esprit» ${ }^{43}$. De même, s'il n'est pas possible de dire pourquoi le devoir oblige, il est souhaitable de se demander ce que la conscience du devoir produit dans l'esprit, en d'autres mots comment l'homme se connaît lui-même à partir de l'acceptation du devoir.

D'abord, l'effort qu'exige le devoir force le sujet à sortir de lui-même, à s'élever au-dessus de sa nature sensible et à prendre conscience de sa propre nature rationnelle. Comme le devoir n'est pas une exigence venant d'une nature extérieure à nous-mêmes, mais qu'il est issu de notre propre raison, de ce qui, en nous, nous rattache à un monde moral supérieur, le devoir ne se présente pas comme une limitation de l'homme et de sa liberté, mais comme la condition d'une révélation de la vraie grandeur de l'homme et de sa liberté. Il prend conscience alors de sa personnalité, "c'est-à-dire la liberté et l'indépendance à l'égard des mécanismes de la nature entière» ${ }^{44}$.

Dès lors, l'homme se découvre non pas seulement comme un sujet empirique, une personne appartenant au monde sensible, ou même comme l'unique sujet de la connaissance de ce monde, mais en tant que sujet du devoir, comme personnalité propre et comme fin en soi. Ainsi, si tout ce sur quoi l'on a quelque pouvoir dans le monde peut être utilisé simplement comme moyen, l'homme seul, et avec lui toute créature raisonnable, est fin en soi. Par le devoir je prends conscience de ma dignité d'homme et de ma supériorité, en tant qu' homme, sur le monde. La nature devient ainsi relative à l'homme, l'objet de son pouvoir d'action sur elle. La nature entière est moyen; seul l'homme est fin en soi.

L'anthropologie morale issue de la philosophie kantienne apporte à l'homme une nouvelle responsabilité vis-à-vis du monde, en faisant de lui le législateur du royaume moral. Mais pour l'amener jusque là, il fallait que fût affirmée la primauté de la raison pratique sur la raison spéculative, et que fût renversée la conception de la destinée humaine que la philosophie occidentale avait héritée des anciens, spécialement de Platon et d'Aristote, pour qui la fin ultime de l'homme se trouvait dans la contemplation du Bien absolu ou de l'Intelligence suprême. Avec Kant, l'action n'est plus déduite de l'être, elle est la condition de la révélation de l'être. C'est donc dans l'étude des conditions de l'action, dans la saisie du devoir comme forme absolue de cette action, que l'homme est révélé à lui-même et qu'il trouve sa vraie grandeur. 
Libéré de la nécessité de trouver la fin ultime de son propre devenir en dehors de lui, l'homme est par Kant ramené à lui-même, à la conscience d'un être rationnel qui est une fin en soi. C'est ainsi qu'il est appelé à dominer la nature à laquelle il échappe par le devoir. Mais en le détachant de cette nature, cette philosophie l'engage dans une recherche incessante de son propre moi, par delà les conditions et les limites de la vie apparente, par delà les déterminations de l'empirisme, dans la prise de conscience d'un devenir humain qui s'ouvre sur l'infini. L'homme moderne est né. 


\section{L'explication de l'obligation et la dissolution du devoir}

Socrate, le christianisme et Kant représentent les trois étapes principales et décisives qui ont permis, dans l'histoire de la philosophie occidentale, la constitution d'une théorie complète du devoir et de la moralité.

Avec Socrate, l'homme découvre que l'interrogation sans réponse du monde le renvoie face à lui-même et à la découverte de son moi. La découverte de la subjectivité dans le "connais-toi toi-même» conduit l'homme à se situer volontairement au centre de son action consciente. Mais cette découverte du moi ne transforme pas la théorie morale qui reste déterminée par l'idée d'une poursuite de fins dont le bonheur de l'homme reste la motivation suprême. Prisonnière de l'eudémonisme, la morale antique demeure relative, l'obligation ne s'en dégage nullement.

Le message chrétien, nourri de morale juive, en donnant un sens et une mission à cette subjectivité naissante, et en ouvrant l'interrogation du sujet moral sur le surnaturel, lui permet de dépasser les limites devant lesquelles la pensée antique s'était arrêtée. Il inaugure ainsi une véritable morale de l'intention. Mais en affirmant la personnalité du sujet, le christianisme l'établit dans une relation, c'est-à-dire une religion, celle qui lie la personne humaine avec la personne divine. L'absolu divin fait de cette relation une obligation, et c'est dans l'idée de création que la philosophie chrétienne trouve finalement le fondement métaphysique du devoir.

Avec Kant, enfin, l'action morale se dégage de toute détermination par l'objet, et l'obligation s'impose d'elle-même par sa relation directe avec la raison pratique. Elle devient la forme universelle de toute morale. Mais dès lors les perspectives de l'anthropologie traditionnelle sont renversées: la découverte de l'homme ne précède pas la révélation du devoir, elle en dépend. Le sujet du devoir est un homme nouveau, plus autonome, mais aussi plus responsable.

Mais en réduisant le fondement de la moralité au seul principe du devoir, Kant introduit une division radicale dans la nature de l'homme. Celui-ci appartient à deux mondes irréductibles: celui de la nécessité et celui de la liberté. D'un côté une nature soumise aux lois du monde phénoménal; de l'autre la moralité souveraine ancrée dans l'infaillibilité de la raison. Les conséquences de cette division ont été d'engendrer, dans les philosophies 
qui vont suivre, d'immenses efforts de synthèse pour essayer de rendre à la nature humaine l'unité perdue. Il fallait pour cela surmonter les contradictions que laissait subsister le kantisme. Certains tenteront de réintégrer la moralité dans la nature, d'autres essayeront de déduire la nature elle-même de la moralité. Mais du fait que le devoir se présentait justement comme la voie de réconciliation de ces deux mondes, tenter de les réunir en dehors du devoir, c'était dissoudre ce dernier en voulant l'expliquer.

Il est difficile d'isoler une notion philosophique de la structure logique et doctrinale dans laquelle elle est née. Dès qu'on tente de la sortir de son contexte, ou du système historique qui la soutient, elle perd sa précision et peut revêtir dès lors une foule de significations plus ou moins contradictoires, ouvrant la voie aux interprétations les plus discordantes. Ainsi en fut-il de la notion de devoir dès qu'elle ne fut plus rattachée à l'apriorisme formel dans lequel Kant l'avait développée.

Pouvait-il, après Kant, exister une morale du devoir qui ne fût pas formelle? Le devoir devait-il être kantien ou n'être pas?

De nombreuses questions ont été soulevées à ce sujet, mais aucune philosophie morale post-kantienne ne pourra la contourner. Si l'essence de la morale est en effet d'être obligatoire, il apparaît logique et nécessaire de dévoiler la nature profonde, l'origine et les mécanismes de l'obligation avant de pouvoir fonder quelque morale que ce fut. Ces tentatives se révéleront cependant impossibles, car elles conduiront presque toutes à des renversements de perspective incompatibles avec la notion même qu'elles tenteront d'expliciter. Soit le devoir disparaît dans une explication qui le nie, soit le raisonnement conduit à des doctrines proches du déterminisme, dans lesquelles sont englouties la liberté et la morale elle-même. Car expliquer le devoir, c'est en effet le soumettre à nouveau à la nécessité d'où Kant avait réussi à le sortir. Le devoir ne peut pas être déduit d'axiomes absolus; il doit s'imposer car il n'est pas démontrable.

La deuxième partie de cet historique de l'obligation présente des thèses qui ont tenté de transformer l'approche kantienne. Or, les philosophies postkantiennes qui se sont aventurées sur le terrain difficile de l'explication du devoir sont toutes tombées dans le piège de sa réduction et de sa dissolution. Toutes aussi, souvent sans s'en rendre compte et en dépit de leurs affirmations sur la liberté, ont rejeté l'anthropologie morale que le caractère absolu du devoir kantien avait inaugurée. L'image de l'homme libre, et responsable de la loi qu'il se donne à lui-même, et qui l'oblige en raison de son universalité, a progressivement disparu devant l'appel aux déterminismes biologiques et sociologiques, ou devant l'affirmation d'une liberté désincarnée qui a pu conduire aux pires excès de l'homme contre l'homme. Voilà pourquoi, face à l'irrésistible tentation que représente l'actuelle course à la prospérité matérielle par une libéralisation générale des moyens de l'action, il est nécessaire de reconstruire une morale fondée sur l'obligation, et ceci par simple souci de l'homme. Le retour du sujet dans la vie sociale l'exige. 


\section{Une explication psychologique}

Nous appelons «explication psychologique» celle qui vise à justifier le devoir en le ramenant dans l'ordre de la nature sans mettre en cause le principe de la subjectivité du sujet. Elle consiste à montrer, d'un point de vue qui se veut scientifique, la nécessité de conserver à l'obligation la primauté et la place centrale que Kant lui avait données, sans recourir pour cela à la raison transcendentale et sans tomber dans le formalisme.

La psychologie, comme science humaine empirique et comme domaine de réflexion sur les comportements, a parcouru un chemin considérable depuis que la philosophie de Kant a marqué de son empreinte la culture européenne. Le XXe siècle, quant à lui, a été si radicalement bouleversé par les découvertes freudiennes, que nous avons souvent de la peine à comprendre comment les concepts de base de la psychologie, y compris celui d'inconscient, s'enracinent souvent dans des démarches plus anciennes. En illustrant notre propos par l'analyse d'un texte antérieur à Freud ${ }^{45}$, nous ne voulons pas méconnaître l'apport actuel de la psychanalyse à la compréhension des comportements individuels, mais démontrer comment, en recourant à une analyse scientifique de la psychè, il est possible de diluer, dans l'objet du déterminisme, l'obligation morale que l'on veut défendre. Un livre publié en 1898, Essai sur l'obligation morale, de Georges Fulliquet, seral'occasion de cette démarche.

Fulliquet part de l'idée qu'il faut soutenir l'obligation morale et en donner une explication qui lui confère sa place légitime dans l'ensemble des connaissances humaines et lui assure son efficacité sur la conduite. Après avoir défini l'homme par la puissance d'auto-détermination qui lui permet de s'extraire du monde soumis au déterminisme, et de s'affirmer comme être libre, doué d'une "force» appelée la liberté, Fulliquet affirme qu'une analyse plus profonde de l'homme révèle en lui une deuxième force qui s'oppose à la puissance de la liberté. Cette force n'apparaît, au moment de l'agir, que dans un seul cas, celui de l'obligation morale. Mais si elle peut se manifester au moment précis de l'agir, c'est qu'elle existe en nous de

45 Les premières publications de Freud sur la psychanalyse date du début du XXe siècle: La naissance de la psychanalyse (Aus den Anfängen der Psychoanalyse) est de 1902. Il semble, aujourd'hui, surprenant que l'on puisse ainsi faire appel a l'inconscient sans tenir compte des apports de Freud. Pourtant la thèse de Fulliquet se tient par elle-même. Elle est aussi d'une autre nature, à la limite beaucoup plus proche du sentiment moral de Rousseau que du déterminisme freudien tentant de rendre compte des pulsions inconscientes. 
façon permanente, sans que nous soyons capables de l'apercevoir d'aucune manière. «Elle existe en nous sans que nous en ayons conscience; elle appartient donc au domaine de l'inconscients ${ }^{46}$.

Cette force-obligation, qui se produit en nous au moment de l'action, révèle les deux caractères essentiels de toute force, à savoir orientation et intensité. L'obligation, dit Fulliquet, pousse toujours le libre-pouvoir vers le bien, du moins tel qu'il est capable de le concevoir, même s'il s'en fait une idée fausse. Cette force possède donc d'abord un caractère de sainteté qui consiste à viser le bien; et comme elle se manifeste de plus comme absolue, puisqu'elle prétend à la supériorité incontestée, elle manifeste les deux caractères mêmes de la divinité, sainteté et absolu. L'obligation n'est donc rien d'autre que l'influence de Dieu en nous.

Elle est la voix de Dieu, certes, mais elle parle cependant à travers une force constitutive de l'homme lui-même. C'est pourquoi l'obligation oblige l'homme envers lui-même et non envers Dieu directement. La liberté ne s'oppose donc pas directement à Dieu, ce qui serait inconvenant, mais à cette force inconsciente humaine, ce qui explique qu'elle puisse parfois ne pas être suivie.

Il semble, à première vue, que Fulliquet tente de revenir à une conception morale pré-kantienne, proche de la théorie chrétienne qui fondait l'obligation sur la nécessité de suivre la rationalité divine en raison de la nature blessée de la rationalité humaine. Pourtant il tente de maintenir ce que Kant avait établi, à savoir l'autonomie totale de la nature humaine, en faisant de l'obligation une force qui, par nature, est totalement humaine.

Ayant introduit l'idée de Dieu dans la définition de l'obligation, l'argumentation demeure en permanence ambiguë, à la limite entre la réflexion philosophique et le raisonnement du théologien. C'est lors de la discussion du problème de l'échec possible de l'obligation que la faiblesse de l'argument se dévoile. "Quand l'homme cède à l'influence de l'obligation, il a le sentiment d'avoir pu lui échapper, il a donc le sentiment de la liberté, et s'il se refuse à subir l'influence de l'obligation, l'absoluité de cette puissance ne lui ôte pas le privilège de l'éviter s'il le veut» ${ }^{47}$. On ne peut éviter d'évoquer ici la discusssion du problème de la grâce dans la théologie d'inspiration luthérienne, selon laquelle la grâce est un don gratuit de Dieu qui est à prendre ou à laisser, mais qui ne dépend en aucun cas du propre mérite de l'homme. Ainsi, face à la force de l'obligation, la force de la liberté n'est jamais créatrice, mais seulement un obstacle sur le chemin du bien.

Certes, ces deux forces contraires pourraient se retrouver dans la poursuite d'un bien commun, par exemple dans une fin à poursuivre en dehors d'elles, mais Fulliquet est trop marqué par la subjectivité autonome inspirée de la

46 George Fulliquet, Essai sur l'obligation morale, p. 91

47 Ibid., p. 104 
théorie kantienne pour qu'il puisse reconstruire une théorie éthique fondée sur l'idée de finalité. L'obligation est sainte, selon lui, non pas parce qu'elle vise le bien, mais parce qu'elle est d'origine divine, à l'instar de la volonté de Kant qui rend l'action bonne parce qu'elle trouve formellement sa moralité dans la raison et l'universel. Il y a donc bien un apriorisme chez Fulliquet, qui n'est pas formel et rationnel comme chez Kant, mais qui s'appuie sur une présence supposée de principes divins dans l'inconscient. C'est pourquoi la force-obligation demande à la force-liberté de cèder à ses injonctions pour garantir la moralité de l'acte. La liberté apparaît ici davantage comme source du mal que révélatrice de la moralité.

Il y a cependant, dans l'analyse psychologique de Fulliquet, des éléments intéressants sur le rôle de l'inconscient que propose l'analyse empirique et que l'on peut rapprocher utilement de l'analyse freudienne du sur-moi, bien que les perspectives dans lesquelles les deux théories ont été construites soient totalement étrangères l'une à l'autre. La force de l'inconscient, dit Fulliquet, perturbe le sentiment de la liberté au moment où il veut se mettre à agir, et c'est ce dérangement qui permet d'en déduire l'existence. Pour la psychanalyse, l'inconscient joue un rôle différent, mais ses résultats peuvent être considérés de façon similaire. L'inconscient remplit la fonction d'une mémoire morale dans laquelle s'inscrivent les impressions marquantes, ainsi que les événements de notre enfance et de notre jeunesse. En particulier, cet inconscient devient le réceptacle d'une certaine image morale, celle que nous conservons de nos parents et de nos éducateurs. Cette image se transforme peu à peu en une sorte de seconde personnalité, un sur-moi, qui joue le rôle de législateur inconscient de nos jugements moraux. ${ }^{48} \mathrm{Ce}$ surmoi intervient dans l'orientation de nos actions et, par le truchement des interdits, des tabous, des limitations de toute nature, se manifeste réellement comme une perturbation de la liberté. L'idée de Dieu, ou d'une force divine en soi, pourrait très bien venir de la projection, dans le sur-moi empirique, d'un moi idéal incarnant une perfection péniblement poursuivie à travers tous les efforts d'ascèse et de maîtrise de soi que le sujet s'impose. Mais, et c'est en cela que la psychanalyse diffère totalement del'analyse psychologique de Fulliquet, la découverte du contenu de l'inconscient conduit justement à démystifier le rôle législateur de ce sur-moi, à lui enlever, en le rendant conscient, tout pouvoir perturbateur et, par là, à supprimer l'obligation morale restrictive qu'il pouvait représenter. L'aboutissement est donc contraire au but que poursuivait Fulliquet, qui était de renforcer le pouvoir de l'obligation en en révélant l'existence cachée.

L'explication psychologique, dans la mesure où elle ne se limite pas à l'argumentation théorique mais propose de s'appuyer sur l'expérience empirique directe, conduit à supprimer la notion d'obligation plutôt qu'à la

48 On trouvera dans J.C. Flugel, Man, Morals and Society, une analyse détaillée de la naissance et de la fonction morale sur sur-moi; Chapt. 9 et 10 
renforcer. La psychologie post-freudienne ne peut retenir l'idée d'une force inconsciente à laquelle la liberté devrait se soumettre pour faire le bien, puisque toutes ces forces dont on reconnaît l'existence dans l'inconscient depuis Freud, sont justement des forces qu'il s'agit de dominer et de vaincre par une meilleure connaissance de soi.

Fulliquet nous permet de comprendre ce que l'obligation n'est pas. Son analyse aura tout de même le mérite de nous faire progresser sur la voie d'une construction plus ouverte et plus efficace de l'obligation. Quand il remarque que l'obligation ne se manifeste qu'au moment où le libre pouvoir veut passer à l'action, il ouvre la voie à une réflexion que nous croyons utile. La découverte de l'obligation peut avoir lieu justement dans la situation où se trouve le sujet au moment d'agir. C'est dans le hic et nunc de l'action morale concrète qu'il faut tenter de rechercher l'obligation qui se révèle. Essayer de la trouver en dehors de cet instant privilégié, c'est faire une extrapolation que ne permettent ni l'observation ni l'expérience.

Faire de l'obligation une force permanente, quels qu'en soient l'origine et le lieu, c'est conduire le raisonnement à la confondre, pour finir, avec une des forces du déterminisme. Pour sauver la liberté sans renoncer à l'obligation, il ne faut pas chercher à comprendre l'obligation en dehors de la situation dans laquelle l'expérience nous la donne. Il faut pour cela tenir compte de tout ce qui compose la réalité de la situation, et non pas seulement le sujet, ou le seul bien poursuivi. Une morale de l'intention pure, comme celle de Kant, ne peut échapper au formalisme. En dehors de ce formalisme, une obligation limitée à l'intention se désagrège devant les forces déterminées du sujet, et la liberté se meurt. Le respect de l'expérience morale nous conduit à rechercher l'obligation dans l'action morale elle-même, dans cet instant où la liberté est agissante, en tenant compte de tous les éléments qui composent la situation dans laquelle l'obligation se révèle. C'est ce que nous ferons au Chapitre suivant. L'analyse de Fulliquet a contribué à développer cette conviction.

\section{Une explication vitaliste}

On retrouve, dans les recherches de Jean-Marie Guyau, la même intention d'échapper à l'idéalisme transcendental de Kant. Dans son ouvrage, Esquisse d'une morale sans obligation ni sanction, (1893), il refuse catégoriquement le formalisme et l'a priori, car l'expérience sur laquelle l'homme doit s'appuyer n'est pas celle de l'universalité de la raison mais celle du sentiment concret et incarné. Or cette expérience montre «que le sentiment 
d'obligation n'est pas lié à la représentation de la loi comme formelle, mais de la loi en raison de sa matière sensible et de sa fin $\aleph^{49}$. Cette matière n'est pas non plus celle d'une loi dont l'intelligence ne saisit que l'universalité, mais celle d'un sentiment qui soit capable de déterminer l'individu à agir. La thèse de Guyau se distingue dès lors non seulement de Kant mais de toute éthique de l'intention qui place la volonté libre à l'origine de l'action. Ce que Guyau recherche, ce n'est pas une loi qui soit capable d'orienter une volonté libre, mais bien ce qui est obligatoire à coup sûr et auquel il n'est pas possible de résister. Comme Fulliquet, et contrairement à Kant, Guyau oppose obligation et liberté. S'il doit y avoir une obligation, elle doit s'adresser à ce qui, en l'homme, n'est pas entièrement libre. L'obligation ne se rattache donc ni à la raison ni à la volonté, mais aux sentiments et aux instincts. Voilà pourquoi l'obligation peut être efficace; et voilà pourquoi également elle ne peut pas être formelle.

Matérialité et finalité sont donc les principes fondamentaux de l'obligation, et les principes nécessaires à son efficacité. La matière de l'obligation est donc cet objet du sentiment qui le satisfait. Quant à sa fin, elle ne peut se distinguer de la causalité elle-même. "Les fins ne sont que des causes motrices habituelles parvenues à la conscience de soi; tout mouvement voulu a commencé aveuglément, parce qu'il présentait moins de résistance; tout désir conscient a donc été d'abord un instinct. La sphère de la finalité coïncide, au moins dans son centre, avec la sphère de la causalité... Ce problème: quelle est la fin, la cible constante de l'action? devient donc, à un autre point de vue, celui-ci: quelle est la cause constante de l'action? $\aleph^{50}$. Mais en donnant ainsi à la finalité les caractères de la causalité, Guyau conduit le raisonnement à réduire la morale à une détermination et, en conséquence, à mettre en cause l'idée même de liberté. Car chaque sentiment, chaque visée morale, chaque but poursuivi peuvent être expliqués par la nature de cette causalité. Dans une telle perspective, l'homme n'apparaît que comme la manifestation irresponsable d'une force causale à laquelle rien ne résiste.

Comprendre la morale, et finalement comprendre l'homme, c'est saisir la nature profonde de cette causalité. Or celle-ci se révèle, selon Guyau, comme la vie elle-même. En l'analysant plus à fond, on découvre que cette vie a deux faces: «Par l'une elle est nutrition et assimilation, par l'autre production et fécondité ${ }^{51}$. Ce sont donc les caractères de la vie qui constituent l'essence de la morale et en donne la définition: «la science qui a pour objet tous les moyens de conserver et d'accroître la vie, matérielle et intellectuelle. Les lois suprêmes de cette morale seront identiques aux lois

49 Jean-Marie Guyau, Esquisse d'une morale sans obligation ni sanction, (16e

50 édit., 1921), p. 58

51 ibid., p. 87

Ibid., p. 101 
les plus profondes de la vie même et, dans quelques uns de ses théorèmes les plus généraux, elle vaudra pour tous les êtres vivants» ${ }^{52}$.

Kant avait substitué à la notion d'homme de la métaphysique classique celle d'être raisonnable. Guyau va substituer à cette notion d'être raisonnable celle d'être vivant, et comme le devoir était pour Kant l'expression de la rationalité, l'obligation est pour Guyau l'expression de la vie. Mais, dit Guyau, on a trop interprété le devoir, jusqu'ici, «comme le sentiment d'une nécessité, d'une contrainte; c'est avant tout celui d'une puissance... A ce point de vue, ..., l'obligation morale se ramène à cette grande loi de la nature: la vie ne peut se maintenir qu'à condition de se répandre» ${ }^{53}$.

Comme pour Fulliquet, c'est antérieurement à la prise de conscience du devoir que celui-ci existe, dans la sphère de ce que Guyau appelle «la conscience spontanée et synthétique», c'est-à-dire l'instinct. Mais tandis que Fulliquet faisait parler Dieu dans cette poussée inconsciente, l'obligation ne se distingue nullement, chez Guyau, d'un instinct vital, d'une force, d'une poussée, d'un élan, celui-ci constituant la causalité de l'action. Pour agir moralement, dès lors, selon les exigences de la vie, l'homme doit se laisser aller à la vie qui s'épanouit en lui; il faut qu'il laisse la vie s'épandre elle-même à travers les impulsions qu'elle donne à ses instincts. Il est donc logique que l'homme se méfie d'une conscience et d'une raison qui pourraient $s^{\prime}$ interposer sur cette marche infaillible de la vie.

Il nous semble intéressant de rapprocher cette poussée spontanée de la vie, à la fois cause et finalité de l'action, du système théorique de l'éthique de la prospérité, dans lequel l'individu n'est valorisé que par sa fonction performante dans un ensemble fonctionnel qui se «maintient et se répand», pour reprendre les termes de Guyau, à savoir la vie économique et la croissance de la richesse. Il y a, mutatis mutandis, une vision similaire de l'homme et de sa moralité, qui est favorisée bien plutôt par l'inconscience que par la conscience, comme l'homo economicus de l'éthique de la prospérité qui bénéficie davantage de son action s'il l'inscrit dans le mouvement général de la croissance que s'il tente d'y échapper, acceptant une sorte de maléabilité du vouloir à ce qui le pousse automatiquement. L'activité économique aurait-elle remplacé la vie?

Tandis que chez Fulliquet le devoir se manifeste au moment où s'affirme l'intention d'agir de la liberté, et que plus il y a de conscience libre plus il y d'intervention de l'obligation, Guyau considère incompatibles les notions de conscience et d'instinct. L'homme a le pouvoir de se libérer des forces aveugles qui le poussent par la connaissance qu'il en acquiert: connaissance scientifique d'abord, réfléchie ensuite. C'est pourquoi l'homme, en se connaissant lui-même, en prenant conscience de ce qu'il est, peut faire disparaître l'instinct et sa puissance déterminante. 
Dès lors, pour Guyau, si la force de la vie se présente comme une obligation contraignante, c'est soit parce que l'homme ne se soumet pas totalement à l'instinct et tente de lui résister, soit parce qu'il n'a pas encore suffisamment développé ses connaissances et sa rationalité, c'est-à-dire, dans un certain sens, qu'il n'a pas encore "dés-instinctivé» son action par la force de sa raison. Ce sont les deux voies possibles pour faire disparaître la nature contraignante de l'obligation: l'une consiste à renoncer à la conscience dissolvante de l'instinct et à n'offrir aucune résistance à la poussée de la vie; l'autre à rationaliser toute action et à supprimer définitivement la force de l'instinct.

Certes il est possible d'accepter que l'obligation disparaisse, dans ce qu'elle a d'impérieux et de pénible, au moment où la spontanéité de l'agent rejoint les grandes impulsions de la vie ou, en d'autres mots, les grandes exigences du devoir. Ressent-on encore le poids de l'obligation si l'on fait spontanément ce que le devoir demande? Mais il est difficile de suivre entièrement Guyau quand il affirme que seule cette attitude est entièrement morale. Opposer la morale à la raison n'est possible que lorsqu'on limite la réalité de l'obligation aux forces inconscientes de l'instinct. Mais si l'obligation disparaît au moment où l'instinct ne rencontre plus aucun obstacle, elle doit par contre se manifester quand la raison s'oppose à sa puissance.

Guyau tente de le nier en affirmant que la rationalité, capable de se conduire elle-même, ne connaîtrait aucune obligation. Et tandis que pour Kant le devoir venait du fait que l'homme demeurait imparfait, ni purement phénoménal ni purement nouménal, mais déchiré par la dualité de sa nature, et que supprimer le devoir aurait impliqué soit de réduire l'homme à n'être qu'une manifestation de la vie, ou que la représentation d'un idéal inaccessible, idéal douloureusement contredit parl'expérience, Guyau pense qu'une telle morale, absolument marquée par la raison, peut être envisagée et qu'elle serait totalement débarassée de l'obligation qui se limite au domaine de l'instinct vital.

Toutefois, même s'il était possible à l'homme d'atteindre ce stade où l'action humaine, entièrement rationalisée, pourrait s'épanouir sans obligation vitale, elle ne pourrait cependant échapper à la nécessité de se donner des lois et des principes. Guyau en est conscient puisqu'il reconnaît que l'homme ne peut s'empêcher de donner à cette morale rationnelle des principes nouveaux qu'il appelle des substituts du devoir. Même débarrassée de la morale instinctive de la vie, la raison se redonne une forme d'obligation à laquelle elle doit se soumettre. Il faut des principes posés et déterminés qui, cependant, «ne peuvent l'être que par hypothèses; il faut donc que je crée moi-même, en définitive, les raisons métaphysiques de mes actes " ${ }^{54}$. 
Après s'être débarrassé de l'obligation instinctive de la vie, la raison se retrouve devant la nécessité de s'obliger à nouveau, mais cette fois par les exigences de sa propre nature. Cette morale rationnelle n'est-elle dès lors pas semblable à celle de Kant? Ce serait le cas si Guyau trouvait dans la forme universelle de la loi la force contraignante de la morale; mais ces substituts du devoir n'ont en fait rien de formel, ils s'expriment concrètement sous divers impulsions d'action appelées idées-forces, fusion des sensibilités, amour du risque dans l'action, et finalement l'amour de l'hypothèse métaphysique sous la forme d'un risque de la pensée.

Même libéré de la poussée de l'instinct, l'homme conserve le besoin d'une réalité extérieure à lui-même dans laquelle il trouve à la fois la justification et l'impulsion que son action nécessite. Guyau, qui visait une morale sans obligation n'échappe pas à la nécessité de reconstruire une morale sur des postulats qui apparaissent cependant très proches des postulats kantiens, en particulier ces risques de la pensée qui se présentent comme une forme d'appel dans lequel la spontanéité et la vitalité de l'homme trouveront à s'épanouir. Mais cet appel n'est-il pas en réalité une autre forme de l'obligation? Et ne se présente-t-il pas comme une exigence essentielle de la liberté justement parce que celle-ci est fille de la raison? Tenter de justifier ces substituts du devoir conduit à y retrouver une origine qui dépend de la raison elle-même. Ne faut-il donc pas en conclure, à l'inverse de ce que Guyau voulait prouver, que toute morale rationnelle est essentiellement obligatoire et que cette obligation est aprioriste et formelle, puisqu'elle trouve son origine dans la raison, et dans la raison seule?

Le raisonnement de Guyau contient, croyons-nous, des éléments qui peuvent permettre d'éviter un simple retour au kantisme. Les hypothèses métaphysiques que sont ces substituts de l'obligation ne sont-ils pas des valeurs, et ces valeurs n'agissent-elles pas sous forme d'appel, et non de poussée déterminante, appel que l'homme se donne à lui-même dans sa relation avec autrui, dans un risque créateur qui fonde ainsi une morale qui respecte la liberté tout en exigeant un engagement? C'est en effet dans une philosophie des valeurs qu'il est possible d'échapper, à nos yeux, à la contradiction dans laquelle le raisonnement de Guyau a paru s'enliser.

\section{Une explication sociologique}

Les sociologues qui ont discuté du problème moral, et particulièrement ceux d'entre eux qui se rattachent à l'Ecole sociologique dont Emile Durkheim fur le fondateur, ont abordé l'étude du phénomène moral dans un 
esprit totalement positiviste. Il faut d'abord procéder à la saisie des faits, à l'exclusion de tout jugement de valeur préalable, et ensuite à l'explication de ces faits en les rapportant à d'autres faits, eux aussi objectivement et positivement observables. Il en ressort une approche que nous souhaitons illustrer par l'analyse d'une oeuvre particulière d'Emile Durkheim, qui a le double avantage de présenter les caractères types de la méthode sociologique utilisée, et de traiter presque exclusivement de la matière de notre recherche historique. Il s'agit de la communication faite par Emile Durkheim à la Société Française de Philosophie le 11 février 1906, sous le titre: Détermination du fait moral. ${ }^{55}$

La première démarche est de savoir comment nous allons reconnaître les faits moraux, par lesquels il sera possible de définir la réalité morale. La première observation montre que «la morale se présente à nous comme un ensemble de maximes, de règles de conduites» ${ }^{56}$. Mais toutes les régles qui prescrivent des manières d'agir ne sont pas morales, telles les règles d'hygiène par exemple. Par contre si nous observons les conséquences de la violation des règles, nous constatons que la violation des règles entraîne des conséquences fâcheuses pour l'agent. Or ces conséquences peuvent soit provenir de l'acte lui-même, analytiquement, soit ne pas dépendre du contenu de l'acte mais lui être directement rattachées, synthétiquement. Si un trouble organique est causé directement par la violation d'une règle d'hygiène, analytiquement, les conséquences que j'aurai à supporter en commettant un meurtre: blâme, punition, exclusion du corps social, etc. ne dépendent pas directement de l'acte lui-même. Dans ce cas le lien est synthétique. Durkheim appelle «sanction les conséquences rattachées à l'acte par un lien synthétique»" ${ }^{57}$.

Or les actes qui entraînent des sanctions sont ceux qui sont jugés en fonction de règles préétablies, selon leur conformité ou non-conformité à ces règles. Mais il y a des règles qui présentent un caractère particulier, à savoir que nous sommes tenus de ne pas accomplir ce qu'elles nous interdisent tout simplement parce qu'elles nous l'interdisent. «C'est ce qu'on appelle le caractère obligatoire de la règle morale. Voilà donc retrouvée, par une analyse rigoureusement empirique, la notion de devoir et d'obligation, et cela à peu près comme Kant l'entendait» ${ }^{38}$. L'analyse des sanctions positives conduit à la même conclusion. La règle morale est donc celle qui se présente comme impérative.

55 Cette communication a été reprise dans un recueil d'articles de Durkheim, collectionné par C. Bouglé en 1924 sus le titre: Sociologie et philosophie. Nous citons d'après une nouvelle édition de 1963.

56 Durkheim, op. cit., p. 59

57 Ibid., p. 61

58 Ibid., p. 62. Cette affirmation de Durkheim: "à peu près comme Kant» porte à confusion. 
L'observation révèle ensuite une deuxième caractéristique, celle de la finalité. Il n'est pas possible de penser, en effet, que l' homme poursuive unc fin uniquement parce qu'elle est commandée; il faut qu'elle lui semble bonne, désirable et désirée. Le contraire serait psychologiquement inadmissible. La désirabilité est aussi essentielle à l'acte moral que son caractère obligatoire. Chaque fois que l'homme obéit à la règle préétablie, il fait un effort sur luimême, mais il sent également que cet effort est désirable et souhaitable. L'obligation est imprégnée d'eudémonisme. C'est ainsi que «nous éprouvons un plaisir sui generis à faire notre devoir. La notion de bien pénètre jusque dans la notion de devoir, comme la notion de devoir et d'obligation pénètre dans celle de bien. L'eudémonisme est partout dans la vie morale ainsi que son contraire ${ }^{59}$.

La recherche d'un équilibre entre obligation et eudémonisme, nous l'avons vu, est l'une des questions clés qui ont animé l'histoire de la notion d'obligation. L'antiquité avait mis l'accent sur le souverain Bien, tandis que Kant affirmait la primauté du devoir. Pour Durkheim, il est absolument nécessaire de maintenir le double caractère de la morale, même si cette dualité peut paraître contradictoire. Il semble qu'il soit en effet impossible d'échapper à cette dualité. Toute définition de l'obligation ou du phénomène moral, nous le constatons, nous conduit à reconnaître soit le dualisme essentiel de la nature humaine, soit l'ambiguîté de sa condition. L'homme n'est pas plus une raison pure qu'un simple phénomène. Ces analyses successives de l'histoire de l'obligation nous font comprendre que la morale ne peut éviter d'être toujours en équilibre instable et de devoir perpétuellement se reconquérir. Le désir et l'obligation y sont constamment mêlés et se contredisent en permanence.

Durkheim justifie sa position en faisant appel à une autre notion de l'expérience humaine qui comporte également cette dualité, à savoir la notion du sacré. "L'objet sacré nous inspire, sinon la crainte, du moins un respect qui nous écarte de lui, qui nous tient à distance; et en même temps, il est objet d'amour et de désir; nous tendons à nous rapprocher de lui, nous aspirons vers lui» ${ }^{60}$.

Ce rapprochement n'est pas fortuit, aux yeux de Durkheim, car il croit qu'il est bien difficile de comprendre la vie morale si on ne la rapproche pas de la vie religieuse. Une telle relation se constate dans toutes les sociétés humaines, mais elle a été particulièrement intense dans la culture occidentale. L'union a été si intime et si longue qu'elle subsiste encore dans la plupart des consciences, même si la religiosité morale et la religiosité théologique tendent aujourd'hui à se distinguer et même à se séparer. C'est de là d'ailleurs que vient un certain sentiment de profanation de la morale quand

59 lbid., p. 64

60 lbid., p. 68 
on ose «la penser et l'étudier avec les procédés des sciences profanes» ${ }^{61}$. Notre brève analyse de l'obligation chrétienne ne dément pas cette opinion.

Pour Durkheim, donc, la morale se définit par l'obligatoire et le désirable, et sa notion se rapproche de celle du sacré. Mais il faut, de plus, déterminer qui sont les sujets de cette morale, c'est-à-dire savoir à qui s'adresse le devoir et pour qui la fin de l'action morale est-elle un bien. Pour le faire, Durkheim se dit contraint «de procéder d'une façon dialectique et d'admettre un certain nombre de postulats, sans les démontrer d'une manière aussi rigoureuse qu'il serait désirable» ${ }^{62}$. Sans nous arrêter pour l'instant à cet artifice méthodologique qui introduit quelque confusion entre la logique des faits et les postulats de la dialectique, le raisonnement se poursuit de la façon suivante.

Le premier postulat dit que tous nos devoirs sont des devoirs «vis-à-vis des consciences; tous nos devoirs s'adressent à des personnes morales, à des êtres pensants». D'autre part, un acte ne peut avoir que deux sortes de fins: moi-même ou d'autres êtres que moi. Or le moi ne peut pas être à la fois le sujet et la fin de la morale, car l'expérience montre, selon Durkheim, que la conscience morale commune n'a jamais considéré comme moral un acte visant «exclusivement la conservation de l'individu». Sujet du devoir, le moi ne peut en être la fin. Mais autrui, en tant qu'individu, ne peut l'être davantage car, s'il est mon égal, pourquoi jouirait-il d'un privilège que je n'ai pas? Entre les individus, «il ne peut y avoir que des différences de degré - les unes en plus, les autres en moins - ce qui ne saurait expliquer la différence de nature qui sépare une conduite morale et une conduite amorale».

Enfin, plusieurs individus additionnés ne pourront former cet autre-quemoi nécessaire pour que ma conduite ait une valeur morale, "car si chaque individu pris à part est incapable de communiquer une valeur morale, une somme numérique d'individus n'en est pas davantage capable». Ainsi une conduite n'aura de valeur morale que si elle a, comme fin, une réalité qui soit à la fois supérieure à l'individu que je suis, et supérieure aux individus que sont les autres. Or, si nous ne pouvons être liés par devoir qu'à des sujets conscients, qu'à des personnes «morales», c'est du moins la prémisse de Durkheim, «il ne reste plus d'autre objectif possible à l'activité morale que le sujet sui generis formé par une pluralité de sujets individuels associés de manière à former un groupe; il ne reste plus que le sujet collectif». Encore faut-il que celui-ci soit différent d' une simple somme quantitative d'éléments qui ne peuvent avoir de valeur morale par eux-mêmes. Il faut donc en conclure, affirme Durkheim, que «s'il existe une morale, un système de devoirs et d'obligations, il faut que la société soit une personne morale

\footnotetext{
61 Ibid., p. 70

62 Idem
} 
qualitativement distincte des personnes individuelles qu'elle comprend et de la synthèse desquelles elle résulte» ${ }^{63}$.

La société se présente donc comme le fondement de la valeur morale de la conduite. Elle a tous les caractères d'une autorité morale qui impose le respect et peut de ce fait être considérée comme fondement du devoir. Mais elle est également «immanente» aux individus qui la composent, c'est pourquoi ceux-ci y trouvent le bien qu'ils poursuivent. Vouloir la société, c'est vouloir quelque chose qui nous dépasse, mais c'est en même temps nous vouloir nous-mêmes. La société est la source à la fois de l'obligation et de la dimension eudémoniste de la morale; elle impose la règle et éveille le désir. Les deux caractères du fait moral deviennent ainsi intelligibles. «En même temps qu'on aperçoit ce qu'ils expriment, on voit ce qui fait leur unité: ils ne sont que deux aspects d'une seule et même réalité, la réalité collectiven ${ }^{64}$.

Ces conclusions appellent une série de commentaires. Tout d'abord d'ordre méthodologique. Le saut de l'observation au postulat devrait être fondé pour que les conclusions auxquelles le raisonnement conduit puissent être convaincantes. Sinon nous parvenons simplement à un nouveau postulat. Durkheim le reconnaît, d'ailleurs, quand il écrit: «On remarquera l'analogie qu'il y a entre ce raisonnement et celui par lequel Kant démontre Dieu. Kant postule Dieu parce que, sans cette hypothèse, la morale est inintelligible. Nous postulons une société spécifiquement distincte des individus, parce que, autrement, la morale est sans objet, le devoir sans point d'attache ${ }^{65}$.

Certes, mais Durkheim entretient ici une confusion dans les termes qui obscurcit son argument. Kant ne démontre pas Dieu, il le postule, et ce postulat n'est pas nécessaire à la compréhension du devoir. Au contraire, il découle du devoir beaucoup plus qu'il ne le soutient. Or il y a une immense différence entre un postulat et le résultat d'une démonstration. Tandis qu'il souligne bien qu'il "postule» la société, Durkheim construit ensuite son raisonnement comme si la société était démontrée. On n'échappe donc pas à l'ambiguïté de la thèse. Il est donc nécessaire de soulever quelques questions. La première est de savoir pourquoi les caractères essentiels de la moralité, obligation et désirabilité, demeurent inintelligibles sans le recours au postulat d'une société prise comme sujet sui generis. Cette question nous ramène au premier postulat, à savoir que nous n'avons de devoirs que visà-vis des consciences, que tous nos devoirs s'adressent à des personnes, à des êtres pensants. Mais quels sont ces «sujets conscients» qui constituent la finalité de l'action morale? L'individu que je suis? d'autres êtres que moi?

En orientant la recherche dans ce sens, l'enquête sur le fondement du devoir se résume en une recherche sur sa finalité. Cette fin doit donc avoir

63 Pour la suite de ces citations: Ibid., pp. 71-74

64

lbid., p. 82

lbid,. p. 74 
un caractère causal, puisqu' elle est à la fois fin et fondement. S'appuyant donc sur le principe selon lequel la cause doit contenir plus que l'effet ou être supérieur à lui, Durkheim affirme que la morale n'a de sens «que si le sujet auquel nous nous subordonnons a une valeur plus haute que nous, individus ${ }^{66}$. Mais le sujet collectif qu'il postule ne convainct pas entièrement car rien ne prouve en effet, en dépit du recours continuel à l'expérience et à l'observation, que ce «sujet collectif» soit la société. Durkheim le reconnaît, d'ailleurs. Il est un autre sujet, dit-il, «qui pourrait jouer le même rôle: c'est la divinité. Entre Dieu et la société, il faut choisir». Mais Durkheim reste indifférent devant ce choix car, positiviste avant tout, il ne voit dans la divinité que la société transfigurée et pensée symboliquement.

Mais la confusion vient surtout de la formulation du problème. En ne s'interrogeant sur le devoir qu'à partir de la finalité de l'action, Durkheim ignore la question du sujet du devoir, c'est-à-dire de la conscience qui est obligée par le devoir. La collectivité pourrait-elle être un tel sujet? Mais alors quelle devrait être sa finalité? L'individu? La société, en tant que sujet sui generis, est-elle un sujet agissant, et peut-elle, à ce titre, être obligée envers ses membres, par exemple en étant «au service» des individus qui la composent? Autant cette question est intéressante à notre époque où les questions de responsabilité respective des individus et de l'Etat sont remises en cause, autant la perspective dans laquelle Durkheim a situé son argumentation évite de pouvoir y donner une réponse. D'une part c'est la société qui fonde le devoir; mais d'autre part c'est l'essence de cette société comme sujet sui generis qui est fondée sur le devoir. Le raisonnement demeure ambigu, aussi, pour échapper à cette ambiguiité, il faudrait postuler une réalité qui soit supérieure à la fois au devoir et à la société et qui fonde à son tour un principe rationnel sur lequel il serait possible de fonder le lien entre devoir et société de façon intelligible. La méthode choisie par Durkheim ne conduit pas jusque là.

Si le raisonnement de Durkheim ne satisfait pas, c'est qu'il a limité sa recherche à la seule explication par la finalité. De ce fait, il n'a pas vu que le devoir, justement parce qu'il n'est pas une détermination, ouvre d'autres perspectives. Car le devoir implique que sa fin ne soit pas nécessairement visée. Il semble, en effet, que pour Durkheim l'homme n'a pas le pouvoir de ne pas choisir la société. Ce qui change totalement la conception du devoir. Si l'homme agissait toujours pour la collectivité et remplissait infailliblement le rôle qu'elle lui impose, comme un organe qui n'a pas d'autres raisons d'être que de remplir la fonction qui lui est impartie, on ne parlerait pas de devoir, mais d'une simple détermination. S'il y a un devoir, c'est que l'homme est libre. Le devoir est donc autant fondé sur le sujet de l'obligation que sur son objet ou sa fin. Ce n'est pas parce que la collectivité est un sujet supérieur aux individus que le devoir existe, c'est parce que nous 
sommes libres. Réduire l'obligation à un seul principe détruit ou l'obligation, qui se transforme en déterminisme, ou la liberté de l'homme, qui est source de sa dignité.

Il n'empêche que l'analyse sociologique a le mérite de souligner la dimension sociale dans laquelle l'homme prend conscience de son obligation. L'homme naît social et naît à la vie morale dans le même mouvement vers l'être, et il est difficile de croire, à la suite de Rousseau, qu'il y ait une existence humaine qui précède l'existence sociale. La philosophie contemporaine ne manque pas d'être sensible à cette dimension de la collectivité et ne commet pas «l'oubli de l'autre» dans la construction ontologique du moi que beaucoup de moralistes du devoir ont pu commettre dans l'histoire récente.

Essentiellement sociale, la vie morale n'est cependant pas exclusivement sociale. Elle exige une société d'êtres libres. Il est donc possible d'observer, antérieurement à la prise de conscience de soi, une vie sociale qui ne soit pas entièrement morale, dans laquelle il y ait une forme d'obligation qui ne soit pas un authentique devoir. La découverte de ces deux sources de la vie morale dans la société représente le grand apport de la philosophie bergsonienne à notre recherche.

\section{L'explication bergsonienne}

L'inspiration de la philosophie morale de Bergson est double. Elle est à la fois vitaliste et sociologique. Mais sa vision est plus large en ce sens qu'elle ne réduit pas la vie à l'impulsion de l'instinct de Guyau, ni la vie sociale au déterminisme auquel conduit l'analyse de Durkheim. Bergson donne de la morale une notion plus large qui éclaire toutes les manifestations particulières au delà desquelles il faut aller la chercher. Morale et vie s'identifient, mais en donnant «au mot biologique le sens très compréhensif qu'il devrait avoir, qu'il prendra peut-être un jour, et disons ... que toute morale, pression et aspiration, est d'essence biologique ${ }^{67}$.

Cette vie est essentiellement un élan, un dynamisme, qui se manifeste par les deux orientations que Guyau avait déjà soulignées et qui sont: d'une part la conservation de la vie et d' autre part son expansion et son dépassement.

67 Henri Bergson, Les deux sources de la morale et de la religion (citations à partir de la 46e edit., PUF, 1946), p. 103 
Biologique, la morale aura donc également cette double orientation, à savoir la préservation de ce qui est et son dépassement.

Bergson part de l'analyse du sentiment d'obligation, dont nous faisons continuellement l'expérience et qui pénètre toute la vie depuis la plus tendre enfance. Pourquoi obéissons-nous? Pourquoi ne jouissons-nous pas de notre liberté? D'où viennent ces habitudes de soumission qui s'inscrivent en nous dès les premiers instants de notre vie? C'est, répond Bergson, que derrière nos parents et nos maîtres, «nous devinions quelque chose d'énorme ou plutôt d'indéfini, qui pesait sur nous de toute sa masse par leur intermédiaire»: la société. ${ }^{68}$

Nous prenons peu à peu conscience qu'à travers nos habitudes, c'est la société qui nous impose notre manière d'agir et qui pèse sur nous, non pas simplement par la somme des habitudes particulières, mais par un poids différent. «Sa pression, comparée à celle des autres habitudes, est telle que la différence de degré équivaut à une différence de nature». Ainsi chaque obligation particulière s'impose à nous comme partie intégrante de ce qu'on peut appeler l'obligation en général. «Et ce tout, qui doit d'être ce qu'il est à l'apport de ses parties, confère à chacune, en retour, l'autorité globale de l'ensemble. Le collectif vient ainsi renforcer le singulier, et la formule "c"est le devoir' triomphe des hésitations que nous pourrions avoir devant un devoir isolé» ${ }^{69}$.

Le poids social pèse sur nous à la manière d'un organisme qui impose ses ordres à chacune des cellules qui le composent. La société oriente ainsi notre action avec une force qui, apparemment, dépasse nos possibilités de lui résister. Ceci vient de la nature fondamentalement sociale de l'être individuel. La solidarité qui nous lie avec les autres hommes vient de ce qu' «un moi se surajoute en chacun de nous au moi individuel. Cultiver ce "moi social" est l'essentiel de notre obligation vis-à-vis de la société». Nous retrouvons ici le «sur-moi» de la psychanalyse, remplissant le rôle de la société et non celui d'une personne idéale, mais jouant un rôle déterminant tout aussi important. Ce moi social, c'est en fait la conscience morale sociale que nous développons en nous et qui nous conduit, peu à peu, à juger de nos actions en fonction de ce que la société attend de nous. La conscience morale est la pression sociale devenue immanente. Celui qui tenterait de s'en libérer ou de s'en distancer, sentirait automatiquement le besoin de s'en rapprocher à nouveau. "Telle est la force qui poussera le criminel à se dénoncer».

Il ne s'agit pas ici d'une exigence rationnelle, mais de la force d'un instinct. La société humaine est semblable au monde des fourmis. Si l'une d'elles était tentée, par un éclair d'intelligence, de ne plus vouloir se sacrifier pour les autres, cette tentation ne durerait que l'instant pendant

Ibid., p. 1

lbid., $\mathrm{p} ; 3$ 
lequel l'intelligence brillerait en elle; mais dès le moment où l'instinct reprendrait le dessus, l'intelligence ne pourrait que se rendre et se convaincre de la supériorité de la force instinctive en affirmant: «il faut parce qu'il faut». C'est donc dans la force de l' instinct qu'il faut chercher la nature d'un impératif absolument catégorique. L'habitude étant justement la façon dont les activités intelligentes imitent l'instinct, "l'habitude la plus puissante, celle dont la force est faite de toutes les forces accumulées de toutes les habitudes sociales élémentaires, est nécessaireent celle qui imite le mieux l'instinct». Dans cette perspective, nous retrouvons dans les sociétés humaines, aussi complexes et spiritualisées qu'elles soient, une intention première de la nature qui tend à conserver la vie par le groupe. C'est pourquoi, pour comprendre l'obligation morale, nous devons sans cesse nous reporter à «ce qu'eût été l'obligation si la société humaine avait été instinctive au lieu d'être intelligente»" ${ }^{70}$.

Cependant, cet instinct social vise toujours une société close, une société tournée sur elle-même. La force de l'instinct nous pousse à maintenir la cohésion sociale, même si le maintien à tout prix de cette cohésion peut conduire l'homme à agir contre l'homme. Cette contradiction se rencontre dans les guerres, par exemple, quand une société exige, au nom d'un absolu social, que l'homme se dresse contre l'homme. C'est pourquoi la morale sociale ne peut prétendre à l'universalité; les guerres montrent, de façon douloureuse, que la morale sociale de l'instinct ne vise pas l'humanité ellemême: «c'est qu'entre la nation, si grande soit-elle, et l'humanité, il y a toute la distance du fini à l'infini, du clos à l'ouvert» ${ }^{71}$. La différence entre les deux objets est de nature, et non plus simplement de degré.

Il y a donc, pour Bergson, une autre morale qui n'est pas seulement une extension de la morale sociale, mais qui est d'une toute autre nature. Il n'est en effet pas possible de passer de l'une à l'autre par une sorte de gradation, en passant par des échelons progressifs que seraient la famille ou la nation. Il faut une rupture, un saut, un dépassement du groupe social. Cette morale de l'humanité est représentée par des sages ou des saints, devenus les témoins ou les modèles de cette morale complète, ou mieux dite «absolue». Or ces saints et ces sages rayonnent un certain attrait autour d'eux. Mais leur témoignage n'a rien de contraignant, il attire plutôt. «Leur existence est un appel. Car tel est bien le caractère de cette autre morale. Tandis que l'obligation est pression ou poussée, dans la morale complète et parfaite il y a un appels ${ }^{72}$.

Ainsi, comme il y a deux morales, celle de la société close et celle des saints ouverte sur l'humanité, Bergson affirme l'existence de deux sortes de motivation, ou de détermination, qu'il hésite toutefois à appeler toutes

70 Pour la suite de ces citations; Ibid., p. 8-23

71 lbid., p. 27

72 lbid., p. 30 
les deux «obligation». Dans la première morale, écrit-il, «l'obligation représente la pression que les éléments de la société exercent les uns sur les autres pour maintenir la forme du tout», dans la seconde morale, «il y a encore obligation, si l'on veut, mais l'obligation est la force d'une aspiration ou d'un élan ${ }^{73}$. Ces deux aspects de la morale et de l'obligation rejoignent les deux tendances fondamentales de la vie, sa conservation et son dépassement. C'est pourquoi ces forces, ces «obligations», sil'on veut, sont données, pour Bergson, dans la réalité avant même qu'on ait tenté d'en rendre raison par l'intelligence.

Mais quel sera alors le rôle de l'intelligence dans cette construction? L'observation montre que les deux morales, close et ouverte, distinguées dans leur nature respective, sont en réalité continuellement mêlées dans la vie des hommes. Il y a donc, entre les domaines où se situent respectivement les deux morales, un champ de contact, un passage, un moment où la morale close s'ouvre, une sorte d'osmose dans laquelle l'aspiration agit surl'obligation et l'obligation sur l'aspiration. Cet "entre-deux» est, pour Bergson, le domaine propre de l'intelligence. «L'obligation qui s'attache à l'ordre est, dans ce qu'elle a d'original et fondamental, infra-intellectuelle. L'efficacité de l'appel tient à la puissance de l'émotion qui fut jadis provoqué, qui l'est encore ou qui pourrait l'être: cette émotion, ne fût-ce que parce qu'elle est indéfiniment résoluble en idées, est plus qu'idée; elle est supra-intellectuelle. Les deux forces s'exerçant dans des régions différentes de l'âme, se projettent sur le plan intermédiaire, qui est celui de l'intelligencen ${ }^{74}$.

C'est donc en vain que l'intelligence tente de fonder l'obligation par ses propres armes, en s'appuyant sur la rationalité de ses principes, puisque ce qu'il y a d'obligatoire dans l'obligation ne vient pas de l'intelligence. Son seul rôle moral est peut-être de corriger les hésitations de l'instinct, au moment où un raisonnement a refoulé une nécessité sentie et subie, afin de la faire revivre et de la rétablir par un raisonnement allant en sens contraire. C'est que, devant la nécessité de la poussée vitale ou de l'élan, toute théorie intellectuelle de l'obligation devient inutile et inopérante; «inutile parce que l'obligation est une nécessité de la vie; inopérante parce que «l'hypothèse introduite peut tout au plus justifier aux yeux de l'intelligence (et justifier bien incomplètement) une obligation qui préexistait à cette reconstruction intellectuelle» ${ }^{75}$.

Des questions doivent être soulevées sur ce rôle de l'intelligence dans la morale bergsonienne. L'intelligence ne fonde pas l'obligation, elle se contente de la reconstruire, par un raisonnement que nous appellerons «d'auto-persuasion", au moment où un raisonnement précédent a mis en échec «l'obligation-nécessité» de l'instinct. Mais c'est justementl'intelligence

Ibid., p. 53

lbid., p. 85-86

lbid., p. 96 
qui, en soulevant des questions, en imaginant, peut-être à tort, des voies différentes de celles que la vie propose, perturbe la force instinctive. C'est ensuite seulement, devantl'incertitude, disons devant la liberté que l'intelligence découvre parce qu'elle n'est plus infailliblement guidée par les habitudes déterminantes que sont les obligations particulières, qu'elle bâtit une théorie lui indiquant à nouveau le chemin de la nécessité préalablement existante. Cette théorie, toutefois, ne peut apparaître que comme une illusion de l'obligation puisqu'elle n'est en fait que la suppression d'un obstacle à la nécessité, et non un fondement justifié de la puissance capable d'orienter notre action. Or, comme l'obstacle ainsi supprimé a été lui-même établi par l'intelligence, celle-ci ne fait au fond qu'anéantir sa propre activité. Une théorie intellectuelle de l'obligation ne serait donc qu'une négation d'une négation, c'est-à-dire la reconstruction par l'intelligence de ce qu'elle avait eu le pouvoir de détruire. C'est donc un rôle bien inutile que joue l'intelligence dans la morale puisque la vie se conserverait bien mieux et avec moins d'efforts si l'intelligence n'existait pas.

Mais quel est l'effet de l'intelligence dans cette mise en échec de la nécessité? En fait, elle met l'agent en situation de liberté, mais Bergson n'insiste pas sur la signification de cette révélation la liberté et de ce que cela implique. Certes il reconnaît que l'obligation ne se conçoit pas sans liberté; «un être ne se sent obligé que s'il est libre, et chaque obligation, prise à part, implique la liberté» ${ }^{76}$. Mais une telle affirmation ne s'applique qu'à chaque obligation séparément, et elle ne nous dit pas si l'obligation globale, le «tout de l'obligation», implique également la liberté ou non. Certes, Bergson ajoute que plus nous descendons des obligations particulières apparentes vers ce tout de l'obligation qui en est le fondement, la base invisible du devoir, «plus l'obligation nous apparaît comme la forme même que la nécessité prend dans le domaine de la vie quand elle exige, pour réaliser certaines fins, l'intelligence, le choix, et par conséquent la liberté». Mais quel rôle joue alors l'intelligence dans la réalisation de ces fins si cette obligation première est située dans un domaine «infra-intellectuel»?

La difficulté vient du fait que deux conceptions de la notion d'obligation se confondent dans la construction bergsonienne. L'une voit dans l'obligation une force qui pousse l'homme à se soumettre aux exigences sociales et qui agit par la puissance des instincts. L'autre désigne cette contrainte particulière que ressent l'intelligence au moment où elle arrive à la découverte de la liberté par la domination des instincts. Il y a confusion dans l'utilisation de la seule notion «d'obligation» pour désigner des forces qui s'imposent à l'agent à deux niveaux différents. Il est donc nécessaire de les distinguer. D'une part reconnaître qu'il existe une force infra-intellectuelle, qui est celle des instincts vitaux et sociaux, et une autre qui agit au moment où la force instinctive a été mise en échec par l'intelligence. Seule cette deuxième 
force devrait recevoir le nom d'obligation. Bergson pourrait l'admettre quand il dit, comme cité plus haut, que l'obligation est la forme que revêt la nécessité quand elle a besoin, pour réaliser certaines fins, de l'intelligence, du choix, et par conséquent de la liberté.

Quant à l'appel des saints et des héros, qui retentit dans le domaine supra-intellectuel de la morale complète et parfaite, Bergson dit qu'on ne peut pas parler à son sujet d'obligation au sens strict. "Il y a encore obligation, si l'on veut» ${ }^{77}$. Cette restriction indique qu'il s'agit d'autre chose, d'une force indéfinie, qui ne peut être confondue avec cette nécessité demandant la collaboration de l'intelligence et de la liberté pour assurer la réalisation de ses buts. L'utilisation du terme "obligation» à ces différents niveaux augmente les difficultés. Ces difficultés doivent être rattachées au postulat de départ selon lequel l'intelligence, ou les idées, n'ont absolument aucun pouvoir sur la volonté. «Aucune spéculation ne créera une obligation ou rien qui y ressemble; peu m'importe la beauté de la théorie, je pourrai toujours dire que je ne l'accepte pas; et, même si je l'accepte, je prétendrai rester libre de me conduire à ma guisen ${ }^{78}$. Pour Kant, la raison avait le pouvoir d'orienter l'action et d'agir sur la volonté, car cette raison était constitutive de l'essence humaine. Le devoir naissait donc des exigences internes de la rationalité. Pour Bergson, c'est l'élan vital qui constitue l'homme et fonde son action. C'est donc lui qui conduit l'homme à agir humainement. L'obligation ne peut donc être, métaphysiquement, que la permanence de ce fondement à tous les niveaux où se situe l'action de l'homme. Elle peut être infra-intellectuelle dans l'instinct, ou émotion supra-intellectuelle dans l'appel des saints. L'homme se sent obligé, même s'il se sait libre, parce qu'en lui demeure en permanence la puissance de cet élan vital qui traverse toute l'activité humaine, consciente et inconsciente. L'intelligence s'illusionne donc en croyant fonder l'obligation. Le but de l'action n'est donc pas un but que je me suis donné à moi-même, mais celui que l'élan vital poursuit à travers le déterminisme social, ma conscience illusoire, ma liberté utopique et la sainteté des héros dont j'entends l'appel. Bergson qui avait fortement affirmé contre Kant la possibilité d'une intuition intellectuelle, a finalement construit une morale dans laquelle, également contre Kant, il dénie à la raison le pouvoir d'être pratique.

L'homme, selon Bergson, s'explique par deux forces qui conditionnent et conduisent l'activité humaine, la poussée et l'appel, forces dont Bergson dit qu'elles «ne sont pas proprement et exclusivement morales, et le moraliste n'a pas à en faire la genèse ${ }^{79}$. Comment peut-il alors affirmer qu'elles fondent la morale? Si l'élan vital n'est pas la source du caractère moral des forces qui conditionnent l'être humain, il faut peut-être chercher

77 Ibid., p. 53. Italiques ajoutées par nous

78 Ibid., p. 45

79 Ibid., p. 98 
ce caractère moral dans une autre direction, dans ce lieu intermédiaire entre le domaine infra-intellectuel de l'instinct social et le champ supra-intellectuel de l'appel des héros, dans ce lieu intermédaire qui est celui de l'intelligence. La nature morale viendrait donc de l'intervention de l'intelligence dans la vie et l'activité humaines, intervention dont Bergson dit justement qu'elle ne peut fonder l'obligation.

S'il y a une morale, un phénomène moral, c'est précisément parce que l'homme échappe à l'élan vital dans ce moment privilégié où l'intervention de l'intelligence met en question l'être donné et fait naître à la fois la conscience et la liberté. Le rôle de l'intelligence n'est donc pas seulement de supprimer les obstacles qu'elle dresse elle-même devant la nécessité vitale, mais également d'intervenir positivement dans la construction qui devient morale au moment même où elle lui communique sa propre nature. L'obligation bergsonienne serait donc une force aveugle et amorale si l'intelligence, et par elle la conscience de l'homme, ne venait lui donner la forme dans laquelle elle trouve sa nature proprement morale. En fin de compte, nous dirons que la morale close est infra-morale, et la morale ouverte est supra-morale; seul apparaît comme authentiquement moral ce domaine dans lequel l'intelligence fait surgir la conscience et la liberté

Mais cela signifie également que la morale est une réalité où tout est remis en question de façon perpétuelle par une conscience toujours en quête de certitude, et une liberté toujours en quête de libération. L'obligation est morale non pas parce qu'elle est une force, car en tant que force elle n'a pas par elle-même de signifcation, mais parce qu'elle agit dans ce domaine où l'intelligence peut lui donner un sens. C'est en définitive l'intelligence, et par elle la conscience et la liberté, qui constitue réellement la morale. 


\section{L'obligation aujourd'hui}

La philosophie européenne, dans la deuxième moitié du XXe siècle, n'a pas construit de grands systèmes éthiques qui aient marqué l'époque. Bien que le nombre de moralistes et de philosophes ayant écrit sur l'éthique ait été fort respectable, il ne semble qu'aucun d'entre eux n'ait défendu de thèses qui aient transformé la vision que la philosophie avait acquise de l'obligation morale, ou contribué à faire progresser la réflexion sur cette notion. L'accent semble avoir été mis sur l'observation des morales pratiquées beaucoup plus que sur la construction de grands systèmes théoriques, et les problèmes concrets de la vie morale ont semblé capter davantage l'attention des penseurs que les constructions abstraites traitant de la vie morale. Les sciences sociales et politiques, plus positives, plus expérimentales et plus proches de l'observation des phénomènes concrets ont joué un rôle plus important dans le développement de la conscience éthique que les grands développements des philosophes. Il y a eu, durant les dernières décennies du XXe siècle, beaucoup plus de Max Weber que de Kant, beaucoup plus d'observateurs de l'évolution des moeurs que d'authentiques moralistes théoriciens.

Plusieurs raisons peuvent expliquer cet état de choses. D'abord le contenu éthique, à la fois douloureux et tragique, des grands événements historiques qui ont marqué l'Europe pendant, et depuis, la deuxième guerre mondiale. Tandis que les grandes guerres «nationalistes» du début du siècle avaient laissé dans l'opinion publique des sentiments, mitigés certes, mais capables de renforcer des idéaux d'hérö̈sme, d'aventure et même de gloire - la multiplication des monuments glorifiant les «morts pour la patrie» dans toute l'Europe en témoigne - l'Europe est sortie de la deuxième guerre dans un tout autre état d'esprit. La fin du nazisme, avec son cortège de violence et d'horreurs, la reconnaissance de l'existence de "crimes contre l'humanité», la menace de l'arme atomique et la pression morale du monde communiste, suivi de l'écroulement politique et moral des puissances européennes victorieuses qui furent entraînées dans des guerres coloniales grevées d'injustice, ont suscité des réactions dans lesquelles les problèmes du désespoir individuel et les questions de culpabilité publique ont tenu une place dominante. Il n'était plus tellement facile, à l'instar de Bergson, d'imaginer des figures de héros et de saints dans un monde douloureusement marqué par les compromissions récentes. Non seulement l'individu se sentait écrasé par l'immensité des crimes révélés, mais il n'arrivait pas à retrouver une conscience de lui-même suffisamment forte pour maîtriser 
une situation sans précédent. La recherche d'idées susceptibles de sécuriser l'individu ont dès lors eu la préférence sur celles qui pouvaient représenter à ses yeux un défi intellectuel capable de le sortir de lui-même. Le recours à l'absurde ${ }^{80}$ ou à l'ambiguïtét permettait de sauver la conscience de l'individu sans exiger de lui qu'il assume la responsabilité du monde. De plus, les grands penseurs n'échappaient pas tous indemnes de la tempête nous pensons ici à la situation ambiguë d'un Heidegger - et l'on sait que ce ne sont jamais les intellectuels privilégiés des époques de restauration qui se révèlent les plus innovateurs. La philosophie n'est pas une discipline des périodes de reconstruction.

La philosophie existentialiste qui a dominé la scène des premières décennies après la guerre avait certes une dimension profondément éthique, à laquelle nous nous référerons d'ailleurs dans les Chapitres suivants. Toutefois, son affirmation radicale et tragique de la conscience individuelle, saisie dans l'incommunicabilité de son intimité la plus étroite, ne favorisait pas l'élaboration de systèmes éthiques exhaustifs. L'expérience vécue, phénoménologiquement saisie, et poursuivie souvent jusqu'à la «nausée»" ${ }^{82}$, tout en révélant le sujet à lui-même - et en ce sens l'existentialisme s' inscrit ouvertement dans la tradition conduisant au renouvellement de la théorie de l'obligation - n'ouvrait pas la voie à des théories pouvant faire école. L'existentialisme ne pouvait pas, par définition, constuire de système auquel des disciples auraient pu s'attacher. L'individualisme volontaire et parfois militant des grands représentants de l'existentialisme n'a pas favorisé leur rayonnement. C'est pourquoi les idées morales de l'existentialisme (nous pensons aux travaux d'un Gabriel Marcel, par exemple ${ }^{83}$ ), en dépit de leur originalité et de leur richesse, se sont répandues dans la réflexion éthique générale sans y apporter de construction rationnelle nouvelle. Il existe donc un héritage éthique de l'existentialisme, mais aucune thèse qui puisse l'identifier. Chaque fois que nous nous référerons à cet héritage, en particulier à certaines idées sartriennes, nous devrons les reconstruire, pour ainsi dire, afin de les intégrer dans notre propre raisonnement.

L'autre tendance philosopohique qui est proche des thèmes de ce travail, le personnalisme, dont Emmanuel Mounier fut en France le représentant le plus militant et le plus connu, n'a pas connu le succès que ses disciples avaient espéré connaître dans les années trente. Pris entre l'individualisme existentialiste et la pensée marxiste, la Révolution personnaliste et communautaire $^{84} n$ 'a pas eu l'impact politique que ses défenseurs espéraient.

80 Albert Camus, Le mythe de Sisyphe (1942)

81 Simone de Beauvoir, Pour une morale de l'ambiguïté (1947)

82 Jean-Paul Sartre, La nausée (1938)

83 en particulier, Gabriel Marcel, Etre et avoir (1935), ouvrage de réflexion

84 éthique qui s'intitule plus précisément: «Journal métaphysique».

4 Emmanuel Mounier, Révolution personnaliste et communautaire (1935), Le personnalisme (1949) 
Certes la guerre avait sapé ses premiers élans, mais le mouvement n'a pas retrouvé son souffle dans les années qui ont suivi. La philosophie de la personne a pourtant joué un rôle important chez de nombreux auteurs, et le mouvement n'est pas étranger à l'écho qu'a reçu dans toute l'Europe les travaux de Max Scheler. Ce dernier est peut-être l'un des rares moralistes du XXe siècle qui aient encore tenté de construire une théorie de l'obligation inspirée de Kant tout en prenant ses distances par rapport à son formalisme, en tentant de mettre l'accent sur la réalité des valeurs. Il rattache en effet l'obligation à l'existence «matériale» et non formelle des valeurs, qui trouvent leur origine dans leur relation à la "personne». Son ouvrage principal (que nous utiliserons plus loin à diverses reprises) s'intitule: Le formalisme en éthique et l'éthique matériale des valeurs. Il représente la première ébauche, très riche et longuement élaborée, d'une grande éthique que son auteur préparait mais qui n'a finalement jamais vu le jour ${ }^{85}$. Il porte le sous-titre significatif de: «Essai nouveau pour fonder un personnalisme éthique». Il contribue utilement à préparer le passage de la prise de conscience du sujet kantien, pur sujet du devoir, à la personne incarnée et concrète, porteuse de la responsabilité morale. L'oeuvre de Max Scheler représente certainement une articulation importante de la pensée dans le développement d'une conception de la subjectivité morale quin'aboutisse pas à un subjectivisme pur et un individualisme absolu. Sa philosophie des valeurs apporte des éléments essentiels au renouvellement de l'idée d'obligation. Nous en mentionnerons certains éléments dans notre propre analyse.

Mais la pensée morale a été principalement occupée, durant ce dernier demi-siècle, par les problèmes de société, de développement économique et de justice. Les interrogations des moralistes ont été concentrées sur l'appréhension des valeurs morales objectives qui dominent la société, plutôt que sur les exigences ontologiques de l'agent moral lui-même et sur l'anthropologie qui en découlait. Que ce soit dans la ligne de la pensée marxiste, celle du structuralisme, ou celle du développement socio-économique concret des sociétés humaines, - avec l'angoisse non exprimée de voir le Tiers Monde partir à la dérive et mettre en danger la société occidentale en voie d'enrichissement - les débats ont été suffisamment animés et urgents pour occuper le devant de la scène pendant de nombreuses années. L'injustice révélée par l'inégalité des richesses dans les sociétés développées et dans le monde, et l'inadmissibilité des rythmes disparates de développement, ont beaucoup occupé les moralistes traitant de ces questions, laissant peu de place à d'autres intérêts. La morale économique, non pas au sens de l'éthique des entreprises, telle qu'elle est envisagée aujourd'hui, mais au

85 Max Scheler, Le formalisme en éthique et l'éthique matériale des valeurs, "Essai nouveau pour fonder un personnalisme éthique», traduit par Maurice de Gandillac (1955). L'ouvrage en Allemand: Der Formalismus in der Ethik, a connu trois éditions durant la vie de l'auteur, en 1916, 1921 et 1926, à partir d'articles publiés dès 1913 dans le Jahrbuchfür Phänomenologische Forschung. 
sens d'une lutte contre l'inégalité des conditions économiques, des organisations sociales et économiques dans les pays développés comme dans le monde, a fait l'objet de nombreux travaux ${ }^{86}$. La recherche a porté sur les valeurs et les conditions objectives qui permettraient l'établissement d' une plus grande égalité des richesses et d'un meilleur partage des moyens de développement. Mais la démonstration rationnelle du bien, de l'idéal même et des conditions de sa réalisation, n'entraîne pas nécessairement l'action de l'agent moral. Or cette question n'a pas fait l'objet d'attention directe. On s'est contenté de présenter les solutions souhaitables que devait poursuivre l'économie, mais on ne s'est pas tellement soucié de la conscience éthique des agents moraux qui devaient la mettre en oeuvre. Il est vrai que les gouvernements démocratiques se doivent, pour survivre, de respecter avant tout les opinions et les comportements de leurs citoyens et ne pas tenter de les conduire en les culpabilisant. «La moralitén'est pas un effet spontané de la démocratie» ${ }^{87}$.

Il en est de même de la majorité des travaux qui ont été poursuivis sur la notion de justice, à laquelle de nombreuses publications ont été consacrées, particulièrement dans le domaine de l'éthique politique. L'oeuvre de John Rawls, Théorie de la Justice $e^{88}$ illustre parfaitement cette tendance. Le succès que cet ouvrage a connu ces dernières années et les commentaires qu'il a suscité en sont la preuve. On y retrouve certes une discussion des notions de devoir et d'obligation, mais elles sont toujours liées à des contenus bien spécifiques qui concernent l'établissement de la justice d'équité. Mais, chaque fois, il s'agit de décrire des devoirs ou des obligations bien définies, au sens des officia de Cicéron, sans interrogation spéciale sur l'anthropologie qui soutient ces contenus. L'éthique politique consiste à élaborer le meilleur modèle possible de société, ou à décrire le fonctionnement des sociétés données, mais rarement à éveiller l'agent moral au sens du devoir, ou à l'appeler à construire sa propre ex istence par une compréhension nouvelle de l'obligation.

Il faut enfin souligner combien notre siècle a contribué, par l'évolution des idées et des moeurs, à l'avènement des droits de l'individu, contribuant ainsi à soulager celui-ci du poids moral que pouvait représenter les valeurs dominantes de l'environnement psychologique et social. Le libéralisme économique a été accompagné d'un libéralisme politique et moral et d'une évolution des moeurs qui ont grandement favorisé le développement de la

86 Le Conseil oecuménique des églises a beaucoup contribué à ces réflexions, tant en Europe qu'aux Etats-Unis. Voir, par exemple: Kenneth E. Boulding, The Organizational Revolution; A Study in the Ethics of Economic Organizations (1953)

87 Jean Marie Domenach, interview dans L'événement du Jeudi, 3 décembre 1992.

88 Voir aussi l'ouvrage de Domenach: Une morale sans moralisme (1992)

Publié en anglais, A Theory of Justice (Harvard University Press, 1971); la version française, revue par l'auteur, est de 1987 
le sujet, et non pas l'individu édulcoré et «indolore», dans le jeu de notre société. Sinon c'est la société elle-même qui risque de connaître son propre crépuscule, semblable à celui que l'auteur observe dans l'histoire du devoir. Le recours à la notion d'obligation morale, que nous développons dans les Chapitres qui vont suivre, a pour seul but d'éviter les conséquences d'une évolution qui pourrait mettre en danger l'être même de l'homme. 
Chapitre 3

\section{Analyse de la morale et connaissance de soi - Les deux dimensions de la morale - (analyse conceptuelle)}

L'important n'est pas de remonter à la racine des choses, mais, le monde étant ce qu'il est, de savoir comment s'y conduire.

Albert Camus

L'homme révolté

C'est le destin permanent de l'éthique que le négatif soit tellement plus clair que le positif.

Hans Jonas

Le principe responsabilité

Les constructions éthiques que nous a laissées l'histoire de la philosophie ne se présentent pas directement comme des sagesses pratiques, des recettes de comportement ou des décalogues de normes morales. Elle visent le plus souvent à révéler les principes qui sous-tendent les données concrètes de la morale vécue, telle qu'elle est proposée à une époque particulière, ou dans une société donnée. Elles visent ensuite à bâtir, à partir de ces observations, un système théorique intellectuellement satisfaisant qui en donne raison. Mais au-delà de la satisfaction rationnelle que donne la compréhension des fondements de la morale vécue, et du progrès de la connaissance qui en découle, le penseur "pratique" tente toujours de 
proposer une philosophie qui débouche sur l'action. La philosophie morale ne peut se contenter de répondre aux seules exigences de la rationalité, elle doit s'épanouir en efficacité. Elle rejoint en ceci les finalités des théories économiques.

\section{Le champ de la recherche morale}

Une délimitation précise du champ de la recherche en philosophie morale ne va pas sans difficultés. Cette recherche demande, pour être authentiquement philosophique, de se situer tout d'abord dans le domaine illimité de la réflexion philosophique en tant que telle. Il faut ensuite que ses méthodes et sa spécificité correspondent à son objet. Kant, par exemple, procède de cette façon pour expliquer sa démarche. Reprenant à son compte la division des connaissances en trois parties que proposaient les philosophies épicurienne et stoïcienne, à savoir la Physique, l'Ethique et la Logique, il écrit dans la Préface aux Fondements de la métaphysique des moeurs: "cette division est parfaitement conforme à la nature des choses, et l'on n'a guère d'autre perfectionnement à y apporter que celui qui consiste à y ajouter le principe sur lequel elle se fonde». Ce principe, explique Kant, est celui de toute connaissance rationnelle qui peut être ou bien matérielle si elle se rapporte à quelque objet, ou bien formelle si elle ne s'occupe que de la forme de l'entendement et de la raison. De ce principe fondamental on déduira donc que la logique est formelle, tandis que les deux autres parties sont matérielles puisqu'elles traitent d'objets déterminés et des lois auxquelles ces objets sont soumis. Mais la Physique et l'Ethique se distingueront à leur tour, car les lois auxquelles elles donnent lieu ne sont pas semblables. Ce seront "ou des lois de la nature, ou des lois de la liberté»." La Physique sera donc, selon Kant, une Philosophie naturelle, l'Ethique une Philosophie morale.

La philosophie contemporaine, stimulée par l'urgence des problèmes actuels et la complexité de ses interrogations, qui fait une place plus grande à l'expérience vécue pour tenter de saisir de plus près, plus concrètement et plus authentiquement la totalité de la «condition humaine», propose à la recherche morale un champ d'investigation moins systématiquement déterminé et moins abstraitement défini que celui de la vision kantienne. L'invasion du champ philosophique, et plus particulièrement celui de la philosophie morale, par les sciences humaines et en particulier par l'économie, ne permet plus de distinguer de façon aussi radicale les lois de la nature des lois de la liberté. Le champ de l'économie est un exemple très spécifique à cet égard, car ce qu'on y considère souvent comme des lois de la nature

${ }^{1}$ Kant, Fondements de la métaphysique des moeurs, Préface, p. 71-72 
(les lois du marché ne sont-elles pas considérées par certaines écoles comme des lois «naturelles» de l'économie?) sont la plupart du temps le résultat d'un choix politique ou d'une action humaine délibérée, appartenant par conséquent au domaine des lois de la liberté. C'est, par exemple, le caractère spécifique du droit positif, et celui de la science politique.

Voilà pourquoi les penseurs d'aujourd'hui manifestent un sens plus aigu de la complexité de la vie et témoignent, dans leurs réflexions, d'un souci plus profond de conserver au maximum et en toutes choses l'unité de l'être. La conséquence en est le besoin d'élargir à l'extrême le champ des réalités et des problèmes qui peuvent faire l'objet d'une recherche morale.

«Il n'y a qu'une philosophie», écrit par exemple René Le Senne, «et elle ne se laisse guère, malgré les nécessités de l'exposition, débiter en parties. Aucun homme ne peut oublier ce qu'il pense quand il passe à l'action, et son action doit réagir sur sa pensée». ${ }^{2}$ "L'extension du champ de la Philosophie morale,» écrit de son côté Georges Bastide, «est telle qu'elle embrasse la totalité de l'humain, avec ses tenants et aboutissants dans la nature et au delà de la nature; ce qui revient à dire que ce champ s'étendrait, si l'on voulait, à l'infini, et qu'aucune mesure de délimitation topographique, comme celles qui ont cours entre les disciplines de spécialités, ne saurait fixer l'aire de sa juridiction". ${ }^{3}$

Non seulement il n'apparaît pas possible, dans une telle perspective, de considérer la philosophie morale comme une partie spécialisée de la philosophie générale, comme la connaissance d'un aspect particulier, intellectuellement «isolable» de la réalité humaine, mais à la limite elle s'étend par nature à tous les champs des connaissances de l'homme, à toutes les disciplines des sciences sociales, et même au delà. Il convient de considérer la réflexion morale comme une tentative de la pensée qui porte, sur la vie et sur l'homme, un regard aussi complet et diversifié que synthétique et unifié. C'est pourquoi la philosophie morale devient en ellemême une philosophie générale, une "Weltanschauung» rationnellement fondée, une vision globale de l'homme dans sa totalité. En elle s'exprime une pensée qui a pris conscience de l'unité essentielle de l'être et de l'agir humains.

«La moralité ne vient pas comme une couche secondaire, au dessus d'une réflexion abstraite sur la totalité et ses dangers; la moralité a une portée indépendante et préliminaire. La philosophie première est une éthique». ${ }^{4}$ Ainsi, comme l'a écrit Francis Jeanson dans son analyse de la pensée de Sartre, la morale devient «la véritable et l'unique question philosophi-

2 René Le Senne, Traité de morale générale, p. 33

3 Georges Bastide, Traité de l'action morale, p. 9

4 Emmanuel Lévinas, Ethique et infini, p. 71 
quen. ${ }^{5}$ Elle concerne dès lors, dans une seule démarche de la pensée et par une seule vision de l'homme, tous les problèmes clés de la vie humaine, l'éthique et l'ontologie, l'action et l'être, «les lois de la nature et celles de la liberté», et à fortiori les lois de l'action sociale et de l'économie, la matérialité et la spiritualité de l'existence de chacun et de tous.

Il n'est en conséquence plus légitime de délimiter de façon arbitraire et abstraite le champ de la recherche morale, ni par une définition préalable de son objet ou de sa méthode, ni par l'exclusion d'expériences particulières ou la réduction du savoir à quelques principes aprioristes de la connaissance rationnelle. Car l'homme ne peut tolérer la division de son vécu en parties séparées, en facultés distinctes ou en substances isolées. La pensée morale est, "par obligation", une pensée essentiellement multidisciplinaire. Car l'homme aspire à une connaissance de lui-même qui respecte l'unité de son «être-agissant". Le sujet se laisse difficilement débiter en parties séparées.

C'est pourquoi, au delà de sa forme plus particulièrement philosophique, la recherche morale peut donner naissance à une nouvelle «anthropologie», puisque son but n'est pas tellement de savoir ce qu'est la morale en tant que discipline rationnelle, mais bien plutôt de comprendre l'homme à travers l'étude des conditions de son action et, dans le domaine qui retient notre attention dans ce travail, les conditions de l'action par laquelle il est le producteur de son propre bien-être. Kant a ouvert la voie à cette nouvelle connaissance de l'homme. Si l'éthique de notre siècle apporte de nouvelles lumières sur les conditions de l'action, elle nous apporte nécessairement de nouvelles connaissances de la nature humaine.

\section{Ambiguïté de l'anthropologie morale}

L'anthropologie à laquelle conduit la réflexion morale n'échappe cependant pas à une ambiguitté qui lui est essentielle. Elle vient du fait que, visant à comprendre l'homme dans sa totalité, elle est conduite à vouloir découvrir les lois de sa liberté dans celle de sa nature, et à interpréter les lois de la nature à travers celles de la liberté. Nous retrouvons la discussion socratique sur les choses divines et humaines, et la méthode d'aborder les unes et les autres.

Le champ du raisonnement de la philosophie pratique ne pouvant être clos, il ne peut, par essence et en raison de l'intention qui l'anime, s'achever dans son ordre propre de raisonnement. Les problèmes abordés ne peuvent être résolus dans le seul ordre de la pensée, car c'est toujours dans l'agir qu'ils doivent aboutir. La philosophie morale demeure donc inachevée,

5 Francis Jeanson, Le problème moral et la pensée de Sartre, p. 27 
ouverte, car elle ne peut conduire la discussion des notions qu'elle aborde dans sa propre logique jusqu'à une conclusion apodictique. Elle ne peut proposer des vérités, mais des questions et des orientations. Y a-t-il d'ailleurs d'autre façon de s'adresser au sujet pour l'appeler à l'être?

Cette ambiguïté inévitable exige, de la part du philosophe, une grande modestie. S'il ne peut répondre aux questions pressantes et concrètes de notre époque que par une nouvelle forme d'interrogation, de nature volontairement intemporelle, sa contribution serait rejetée comme toutes les formes de sophisme l'ont été au cours de l'histoire, et son effort resterait incompris et vain.

L'ambiguïté vient donc du fait que la réalité qui constitue son centre d'intérêt et qui forme l'objet direct de son analyse, l'action morale, est une réalité à faire, une réalité qui n'existe pas encore. La philosophie morale peut tenter de décrire l'action morale, mais elle n'aura jamais la certitude que l'objet de cette description existe, ou pourra même exister. En conséquence, nous devrons reconnaître que s'il existe une vérité de la philosophie morale, elle ne dépend pas de la rationalité de son propos ou de la rigueur logique de son raisonnement, mais de l'engagement concret de l'homme dans l'action. Ainsi c'est l' homme lui-même, en tant que sujet, dans l'action concrète par laquelle il construit son existence individuelle et sociale, qui achève la philosophie morale.

La notion d'obligation morale est essentiellement une notion de cette nature. Nous avons vu, dans le Chapitre précédent, Deuxième Section, comment la raison ne pouvait éviter de dissoudre la notion d'obligation quand elle tentait de soumettre les objets de la philosophie morale aux lois de l'objectivité. Le devoir disparaît quand on tente de l'expliquer, comme la liberté s'estompe devant les lois de la nature. En expliquant l'obligation, ces philosophies n'appellent pas l'homme à l'action, mais l'invite à se «laisser agir» et à se soumettre. Par contre, nous devons nous souvenir de Socrate qui, en refusant de conduire ses interlocuteurs vers une conclusion logique définitive, les orientait vers une prise de conscience d'eux-mêmes.

C'est dans une telle perspective que nous voyons l'utilité d'une anthropologie morale pour traiter du problème de l'éthique dans la vie économique actuelle. Or, en affirmant son ambiguité essentielle, il est possible de montrer comment cette anthropologie n'apporte pas une connaissance empirique de l'homme, un «savoir» objectif sur sa nature et son comportement, qui puisse éventuellement être utilisé pour faire son bonheur ou le manipuler au bénéfice d'intérêts particuliers, mais se propose au contraire comme l'occasion d'une prise de conscience de l'homme par lui-même. C'est une des questions essentielles de ce livre. En remettant en question les critères de son propre jugement sur lui-même, l'homo economicus de cette fin de siècle doit avoir l'occasion de réancrer la conscience qu'il peut acquérir de lui-même dans un homo total, - le sujet responsable -, ouvert à toute la complexité de sa richesse morale, spirituelle et transcendante. Ceci 
paraît être une entreprise suffisamment valable pour ne pas craindre les difficultés méthodologiques de cet effort d'anthropologie nouvelle. Les questions de datent pas d'aujourd' hui, ${ }^{6}$ mais il est évident que nous devons les renouveler à mesure que notre interrogation sur l'homme se fait plus pressante.

\section{Les concepts de la philosophie morale}

Si, à la suite de Socrate, nous admettons que l'homme doit se découvrir lui-même, comme sujet de l'action, dans l'analyse des notion morales par lesquelles il justifie cette action, nous devons supposer que, d'une façon ou d'une autre, il est lui-même présent, impliqué ontologiquement, du moins en puissance, dans les notions qu'il analyse. Le fait que le champ de la recherche morale ne puisse pas être délimité de façon précise, que les problèmes soulevés par cette recherche demeurent insolubles dans l'ordre de la raison théorique, que les notions analysées ne puissent faire l'objet d'une connaissance objective définitive, révèle que les concepts utilisés par la philosophie morale n'épuisent pas totalement la réalité qu'ils sont supposés exprimer, et que cette réalité les dépasse.

Or ce qui dépasse par essence le contenu du concept, c'est justement le sujet, l'agent, l'auteur de l'action. L'action morale ne peut être saisie logiquement dans sa réalité par une simple analyse conceptuelle puisqu'elle n'existe, dans les faits, que par l'action de l'agent qui l'actualise. Les concepts moraux auront donc cette nature particulière de ne pas pouvoir être soumis entièrement au pouvoir de la raison, ou de ne pas satisfaire les exigences de l'objectivité, encore moins de la science expérimentale. Il n'est donc pas surprenant de voir avec quel empressement les sciences expérimentales, en particulier celles qui se veulent «exactes», tentent d'éliminer de leur champ d'intérêt les concepts des sciences dites morales, par peur méthodologique d'être comme emprisonnées dans un manque insupportable d'objectivité.

Ceci est particulièrement évident en ce qui concerne les concepts de la morale. Ceux-ci ne peuvent être réellement compris par l'entendement que si celui-ci reconnaît en eux un double contenu. D'une part ils doivent exprimer un contenu objectif - contenu sans lequel il n'y aurait aucune connaissance qui soit communicable, aucun dialogue possible à leur sujet, ni même aucune recherche rationnelle - et d'autre part ils doivent impliquer le sujet, sans lequel l'action ne pourrait être actualisée, c'est-à-dire sans lequel la morale n'aurait aucun rapport avec la réalité.

${ }^{6}$ Le livre de Peter Drücker, The End of Economic Man, date par exemple de 1939. 


\section{Deux exemples nous permettent d'illustrer ce problème.}

Prenons d'abord le concept de triangle. Nous constatons, par analyse, que le contenu que nous percevons et comprenons du concept correspond exactement et pleinement à une réalité précise, donnée ou construite, qui est celle d'une "figure plane ayant trois côtés et trois angles dont la somme est égale à deux droits». La connaissance du triangle résulte du développement, par analyse intellectuelle, de toutes les propriétés de la figure. Ce sont les propriétés de la figure qui déterminent la connaissance que nous pourrons en avoir; elle ne dépendra en rien d'un sujet, connaissant ou agissant. Le concept n'est donc pas ambigu, il est "pleinement» objectif et universel, car il implique une complète assimilation de toutes les potentialités de l'objet réel dans l'objet connu. Nous parlerons dans ce cas d'un concept-objet.

Mais prenons comme deuxième exemple le concept d'amitié. Il exprime une certaine forme de relation entre deux personnes. Nous pouvons, certes, rendre objectifs certains aspects formels de cette amitié, en préciser certaines caractéristiques et par là la distinguer d'autres formes de relation entre des personnes, la haine, l'indifférence ou l'amour. Mais le concept d'amitié n'exprime pas uniquement le principe d'une «relation», il implique aussi, nécessairement, les personnes qui sont en relation d'amitié, et qui font que l'amitié a, dans le cas de deux personnes individuelles, une signification et une nature bien spécifiques et, dans un autre cas impliquant d'autres personnes, des potentialités tout à fait différentes. L'amitié entre deux frères, entre un homme et une femme, un fils et son père, entre deux peuples, entre «moi» et "toi», sera chaque fois une amitié concrètement différente. Il y a toujours, dans la réalité, quelque chose qui échappe à la définition, car l'amité «en général» ne peut jamais exprimer tout ce qui se vit dans une amitié «en particulier». C'est pourquoi la raison ne peut jamais donner une définition de l'amitié capable de recouvrir toutes les amitiés possibles, car il n'y a pas de concept suffisamment englobant pour tenir compte de tous les sujets susceptibles d'être en relation d'amitié sur cette terre. Le sujet, en effet, n'est jamais objectivable, et encore moins universalisable.

L'être du triangle est entièrement assimilé par le concept. L'être du sujet, qui est constituant de la notion d'amitié, échappe toujours au concept car il n'est pas objectivable, et encore moins réductible à des critères quantitatifs. Nous appellerons les concepts de cette nature, non pas des «conceptssujets» - car ce serait une contradiction dans les termes - mais des conceptsimpliquant-le-sujet. L'amitié n'est pas un sujet; nous pouvons donc la conceptualiser; mais l'amitié ne se comprend pas par la seule analyse de ses propriétés comme le triangle: elle n'est totale et réelle que par le sujet. Il ne suffit pas de connaître l'amitié pour la comprendre, il faut la vivre.

Les concepts de la philosophie morale sont essentiellement des «concepts-impliquant-le-sujet». Leur analyse ne peut laisser le sujet indifférent puisqu'il se découvre lui-même, en tant que sujet, à travers l'analyse ellemême. Il y a en effet, dans la conceptualisation de la philosophie morale, 
une dimension qui dépasse le simple exercice de la rationalité et qui entraîne une prise de conscience de soi. Un peu comme l'auto-analyse psychologique permet, en pénétrant progressivement dans l'inconscient, non pas de décous vrir ce qu'il contient objectivement, mais de se prendre soi-même en main au delà de la compréhension des "Mots pour le dire»", afin de vivre dans un meilleur équilibre et en accord avec soi-même.

C'est dans cette perspective, d'ailleurs, qu'on pourrait refaire avec Socrate l'analyse des diverses notions morales à laquelle il conviait les Athéniens. Parler sur le courage, n'est-ce pas inviter celui qui tente d'analyser la notion de courage à être plus courageux lui-même, ou à découvrir en quoi il manque peut-être de courage? Certes, on peut peut-être avancer l'idée qu'un parfait couard peut admirablement «connaître» le courage comme concept, et ne rien changer à sa façon de vivre. Bien qu'une telle conclusion paraisse impossible aux yeux de Socrate lui-même, pour qui la vertu résulte de la connaissance du bien, nous ne pouvons pas nier que le lâche, connaissant le courage, se connaît mieux lui-même comme noncourageux et que cette connaissance l'oblige à se repenser lui-même en fonction d'une valeur, à savoir: «est-il préférable d'être courageux ou non?». Car il ne manquera pas de se dire: «je suis lâche, certes, mais je sais que le courage est possible». Et même si, connaissant le courage, il choisit d'être lâche, il manifeste concrètement sa liberté. Car l'homme n'est pas courageux malgré lui et doit, pour faire acte de courage comme de lâcheté, connaître à la foís le courage, la lâcheté, et soi-même. Le lâche ne devient pas courageux par la simple connaissance du courage mais, ayant acquis cette connaissance, il ne pourra plus être lâche dans l'indifférence.

Le même raisonnement peut être appliqué aujourd'hui au concept de justice. La simple affirmation du besoin de justice et la connaissance de ses conditions ne suscitent peut-être pas automatiquement des comportements justes chez les individus. Mais il n'en demeure pas moins vrai que les individus connaissant la justice ne peuvent pas l'ignorer dans l'autoévaluation de leur propre comportement, et ne peuvent certainement plus en faire abstraction dans l'indifférence. On imagine facilement l'impact qu'une telle approche peut avoir sur les valeurs morales sous-jacentes au développement de la liberté économique contemporaine.

La recherche morale qui s'appuie sur l'analyse de ces «conceptsimpliquant-le-sujet» conduit donc nécessairement à une prise de conscience de soi. Le retour du sujet responsable est à ce prix. C'est là, croyons-nous, le sens profond de la recherche de Socrate que nous devons renouveler. A travers toutes les définitions que Socrate essayait de faire naître par ses questions et d'établir par le dialogue, l'homme lui-même était le seul but qu'il poursuivait. A la limite, nous pouvons suggérer que toute la philoso-

7 d'après le titre du roman de Marie Cardinal, Les mots pour le dire, qui relate l'histoire d'une psychanalyse. 
phie socratique et toutes les analyses conceptuelles des dialogues aboutissent au «connais-toi toi-même». De même, notre recherche doit contribuer à resituer le sujet au centre des comportements sociaux et politiques.

Dans la confusion des théories qui nous servent à justifier notre course irrationnelle à la richesse et au bien-être, la présente recherche sur l'obligation morale doit contribuer à ce que l'homme ne soit pas perdu de vue, et que ses valeurs fondamentales ne soient pas ignorées. Renouveler inlassablement cette démarche, c'est rappeler à la conscience du chercheur, mais aussi à la conscience de l'homme agissant, que c'est de lui-même et de sa liberté que dépend l'actualisation des solutions aux problèmes que suscite l'analyse. Si la recherche morale part d'une connaissance, elle ne conduit pas à des connaissances nouvelles dans l'ordre de la raison, mais elle s'achève dans celui de l'existence humaine. La réponse aux questions morales que soulève la crise de la condition humaine ne se trouve pas dans une explication objective, dans une nouvelle théorie économique ou politique, rêve utopique que l'on serait tenté ensuite d'imposer à une société plus ou moins rébarbative, mais dans la transformation du sujet lui-même par l'action responsable qu'il décide d'entreprendre. La recherche morale ne conduit pas à une explication des choses, mais à une conquête de soi.

\section{La double dimension de la morale}

Si le champ de la recherche morale ne peut être délimité avec précision, et s'étend en fait à tout le domaine de l'action humaine, si l'anthropologie morale se présente comme une connaissance ambiguë, si les concepts de la philosophie morale sont des concepts mixtes et problématiques contenant à la fois une exigence d'objectivité et une dimension inévitable de subjectivité, la notion de «morale» rencontrera les mêmes tensions.

Nous parlerons à son sujet d'une double dimension. Nous devons en tenir compte et montrer tout d'abord en quoi la morale est «subjective», et ce que cela implique, et en quoi elle est «objective», et quel rôle cette objectivité joue exactement.

L'action humaine est au centre du problème moral. Nous parlerons à son sujet «d'action morale», non pas parce que nous voudrions isoler une action spécifiquement morale de l'action humaine en général, ou que nous souhaiterions suggérer qu'il puisse y avoir des actions humaines qui ne soient pas morales, mais parce que notre analyse conceptuelle s'attachera plus particulièrement à révéler la signification exacte du caractère «moral» de l'action. Ce que nous distinguons, en fait, ce sont les deux dimensions, subjective et objective, que l'action doit avoir en tant qu'elle est morale. Notre approche consiste ici à analyser le problème de l'action humaine par le moyen de la double signification de l'adjectif «moral». 


\section{L'adjectif «moral» et ses formes négatives}

II n'est pas facile de saisir de façon précise la signification de l'adjectif «moral» en partant de sa forme positive. Son utilisation courante, d'abord, couvre des domaines très divers. De façon générale, le terme moral se dit de tout ce qui concerne les moeurs, du sens moral des agents individuels à la nature de l'impératif qui se trouve au coeur de l'obligation, mais il concerne également l'éthique dans ses deux acceptions: d'une part en tant qu'étude philosophique des principes fondamentaux qui sous-tendent le bien et le mal; d'autre part en tant que système cohérent de jugement du comportement humain. L'adjectif "moral» qualifie de plus ce qui est conforme aux bonnes moeurs, ce qui concerne l'action et le sentiment en tant que distinct du rationnel et du logique, et il couvre finalement tout le champ de l'intellectuel et du spirituel en tant qu'opposé au matériel et au physique. L'expression française «sciences morales», qui traduit l'allemand Geisteswissenschaften, et l'anglais social science ou humanities, est significatif à cet égard. Elle qualifie une certaine classe des connaissances de l'homme, dans sa nature spirituelle, culturelle ou sociale, mais n'implique pas que ces connaissances portent sur des problèmes spécifiquement moraux. En ce sens, les sciences «morales» s'opposent aux sciences «physiques", mais ne sont pas morales au sens où nous entendons ce mot dans ce travail.

D'autre part, même quand l'utilisation de cet adjectif est spécifiquement réservée au champ de la recherche morale, elle ne demeure pas sans confusion. Le terme n'est pas exempt d'ambiguiité, même dans la littérature philosophique. Le Vocabulaire technique et critique de la philosophie, de André Lalande, par exemple, met justement en garde contre une confusion possible, et souligne que ce terme présente «au plus haut point la confusion du "constatatif" et de l'appréciatif, du jugement de fait et du jugement de valeur» ${ }^{8}$. Nous devons donc mettre en lumière l'origine de cette équivoque si nous voulons éviter que notre analyse conceptuelle soit faussée dès le départ.

Tournons-nous pour cela vers les formes négatives de l'adjectif «moral». La forme négative peut parfois être révélatrice du sens des mots plus clairement que les analyses les plus approfondies. Il ne s'agit pas ici d'une dialectique de la thèse et de l'antithèse, mais tout simplement d'un moyen par lequel la signification d'un terme est révélée, et non pas transformée, par l'analyse de sa négation. Ce procédé peut être comparé à l'opposition de la lumière et de la nuit. Pour celui qui vit dans la lumière, la connaissance de la nuit apporte une perspective nouvelle sur le phénomène propre de la lumière et sur son importance dans la vie. L'aveugle né, lui, ne pourra jamais

8 André Lalande, Vocabulaire technique et critique de la philosophie, p. 654 
comprendre totalement ce que la nuit dans laquelle il est enfermé représente, puisqu'il n'est pas en mesure de connaitre la négation de la nuit qu'est la lumière. Il faut toujours sortir de sa caverne pour savoir ce qu'on y vit.

Il existe deux termes négatifs de l'adjectif moral. On peut dire en effet d'une action qu'elle est $\boldsymbol{a}$-morale, ou qu'elle est im-morale. Nous croyons que c'est en révélant ce que ces deux négations nient de la notion positive que nous en comprendrons la signification complète.

Le préfixe négatif «a-» signifie la privation, la suppression de la caratéristique essentielle comprise dans le terme positif. Sera donc dit amoral ce qui est privé de caractère moral, ce qui se distingue de moral par la suppression du sens. Par contre le préfixe négatif «in-» renferme une négation par opposition ou renversement de sens. La signification du terme positif n'est pas supprimée, elle est inversée, elle révèle son contraire. Ainsi, tandis que l'im-moral implique une bipolarité morale, un pôle positif et négatif de la même signification, l'a-moral lui, exclut toute polarité; c'est l'indifférence.

Ce qu'il faut tenter de cerner de façon plus précise, maintenant, est le contenu positif du terme «moral». N'y a-t-il qu'un seul contenu, dont la signification peut être d'une part supprimée par une négation et renversée par l'autre, - donc le terme aurait une signification univoque, - ou faut-il y reconnaître deux contenus distincts, deux significations correspondant aux deux négations existantes?

Il est évident, tout d'abord, que le contenu positif du terme moral, dont la négation implique une suppression du sens, doit avoir sa signification en lui-même, puisque sa négation en entrâne la disparition. L'a-moral étant le non moral, il faut donc que ce qui est moral se présente comme un fait, comme l'objet d'une constatation. En ce sens, le «moral» est, ou il n'est pas. Nous pouvons donc voir dans l'opposition «moral - a-moral» une affirmation de nature constatative; c'est un jugement de fait. Nous dirons donc d'une action qu'elle est morale dans la mesure où elle comprend cette caractérisitique fondamentale sans laquelle le «moral» n'existerait pas. Car l'a-moral ne peut pas être moral, et sa nature est telle qu'il ne peut devenir moral. Le moral n'est pas du même ordre que l'a-moral.

L'autre opposition «moral - im-moral» implique par contre une référence à un troisième terme par rapport auquel le moral et l'im-moral peuvent être repérés et jugés de nature opposée. Nous pourrons dès lors dire que le moral et l'im-moral sont en rapport avec un ordre identique et commun. L'immoral ne se comprend comme tel que parce qu'il pourrait être moral, mais ne l'est justement pas. La négation est comprise dans ce par quoi l'affirmation est connue. L'im-moral se définit donc par ce que le moral devrait être. L'opposition «moral - im-moral» est donc de nature «appréciative», et non plus factuelle, et peut faire l'objet d'un jugement de valeur. Dans ce cas le moral ne se définit pas par lui-même, mais en relation avec un troisième terme qui sera la «valeur». L'action morale est donc celle qui 
affirme une valeur, l'action immorale celle qui la nie. Les deux actions seront jugées à l'aune du même troisième terme, la présence ou l'absence de la valeur.

C'est pourquoi nous pensons qu'il y a deux significations de l'adjectif moral, qui apparaissent de nature différente quand on en analyse le rapport avec ses négations. Notre tâche est maintenant de montrer comment ces deux significations correspondent à ce que nous avons appelé plus haut les deux dimensions de la morale, c'est-à-dire la présence de l'objectivité et de la subjectivité dans la notion de «la morale». Pour cela nous devrons déterminer quel est le contenu qui fait l'objet d'un jugement de fait, quand le moral se distingue de l'amoral, et celui qui fait l'objet d'un jugement de valeur, quand le moral se distingue de l'immoral.

Observons encore que le langage courant confond souvent les notions d'amoral et d'immoral, les prenant l'un pour l'autre et mélangeant leur signification. Cette confusion nous semble avant tout provenir du fait que ce qui est «moral» est d'abord défini positivement, univoquement, préalablement à l'interpétation de ses formes négatives. Les deux négations sont alors difficiles à distinguer. Notre approche par la forme négative nous permet, semble-t-il, d'éviter cette ambiguïté. 


\section{MORAL - AMORAL : Le fait moral et ses implications}

Selon la première signification de l'adjectif moral, l'action doit avoir une caractéristique, ou une qualité essentielle telle que la suppression de cette dernière entraîne la disparition complète du caractère moral et enlève à l'action ainsi caractérisée tout rapport quelconque avec la morale.

Si cette caractéristique est essentielle à l'action morale, il n'est pas possible de la rechercher en dehors de l'action elle-même, c'est-à-dire dans une cause extérieure ou un critère qui s'ajouterait à l'action, et qui ne proviendrait pas d'elle-même. Certes, il est impossible de penser l'action, en tant qu'action, sans savoir qu'un être agissant s'y manifeste. L'action et sa source, l'être agissant, ne sont pas une seule et même réalité. C'est d'ailleurs la conscience de cette distinction métaphysique qui a conduit Maurice Blondel à rechercher dans la notion d'un "pur agir», l'origine explicative de l'action en tant qu'action, qui ne pouvait être saisie au niveau de l'activité humaine que par des causes secondes. ${ }^{9}$ Mais notre recherche ne porte pas avant tout sur l'agir en tant qu'agir, mais bien sur l'action en tant que morale.

Si l'action ne peut être considérée comme morale que dans la mesure où sa caractérisitique essentielle est en elle-même, et non dans une cause extérieure, elle ne sera telle que si l'être agissant, source ou cause directe de l'action, la marquera de son intention et de sa nature. Nous dirons que l'action est morale quand elle ne peut pas être causalement séparée de l'agent et quand elle ne se comprend que par lui. Par contre, dans la mesure où cette dépendance directe de l'agent n'est pas apparente, où des causes extérieures interviennent, seules, et que l'action ne peut être directement imputée à l'agent, nous dirons que cette action est a-morale. Elle est pour ainsi dire sans agent.

Ce lien essentiel de l'action et de l'agent doit cependant être interprété du seul point de vue de la philosophie pratique, et ne pas dépendre de considérations d'ordre métaphysique. Il ne s'agit pas ici de décrire ce que

9 Voir Maurice Blondel, L'action, 2 vols., 1936-37. Maurice Blondel, partant de l'analyse de la causalité dans l'action humaine, remonte à la notion d'un «Pur Agir", c'est-à-dire à Dieu, en qui il voit la source du caractère moral de l'action humaine. C'est donc en fin de compte dans une ouverture métaphysique de l'agir que Blondel trouve la source de la moralité. Quant à nous, dans cette analyse conceptuelle, nous ne remontons pas au-delà de l'agent humain qui est présent dans son acte. 
les choses sont en elles-mêmes, ce que l'action est comme réalité de l'agir, mais de comprendre en quoi elle peut être dite morale, par quel processus elle acquiert sa caractéristique proprement morale. L'identification de l'action et de l'agent est donc une identification morale. Elle n'est d'aucune façon une réduction métaphysique de l'effet à sa cause.

Nous avons dit, plus haut, que les notions de philosophie morale révélaient, de par leur ambiguïté, la présence du sujet dans la perception que nous avons de la réalité morale. L'amitié ne se comprend totalement que dans la relation concrète de deux amis, pris dans leur individualité subjective. Nous dirons donc, à ce niveau du raisonnement, que l'action morale n'est authentiquement morale que dans la mesure où elle implique le sujet, où elle reflète la subjectivité de l'agent. En d'autres mots, c'est la présence du sujet dans l'action qui fait que celle-ci est morale. Sans ce sujet, sans son intervention dans l'action elle-même, sans cette présence non-objectivable essentielle, l'action serait a-morale, c'est-à-dire sans rapport aucun avec l'ordre moral. C'est la subjectivité de l'agent qui rend son action morale.

Cette présence essentielle de la subjectivité dans l'action constitue la première dimension de la morale. Cette dimension est fondamentale, absolument essentielle, puisque, comme nous l'avons vu par l'analyse de la négation, son absence enlève à l'action tout caractère moral. Elle nous permet de porter sur l'action un jugement moral «de fait», c'est-à-dire déclarer qu'elle est réellement d'ordre moral, indépendamment de son contenu ou de sa valeur. Est moral, ressortit à l'ordre moral, tout ce que je fais en tant que sujet; est a-moral, dépourvu de toute connotation morale, toutes les choses, objets ou actions, dans lesquelles moi, ou un autre être humain, n'est pas impliqué en tant que sujet.

Cette affirmation de la nécessaire subjectivité que doit revêtir une action pour être morale ne s'appuie encore que sur des considérations théoriques et formelles. Nous la déduisons pour ainsi dire a priori de la simple analyse des notions. En nous appuyant sur l'opposition «moral - a-moral» , qui est une négation par suppression de la caractéristique essentielle, nous ne pouvions conclure que par une spécification formelle de la moralité. Le propre de la forme, en effet, est de ne pouvoir être renversée ou transformée. Le contraire de la forme est toujours une absence de forme.

Mais quand nous parlons de forme, ici, nous avons à l'esprit la «spécification formelle» de l'acte, et non pas la signification que revêt la forme dans la pensée kantienne. Le devoir est la forme de la raison pratique, puisque selon Kant, il n'est pas possible de déduire les règles du comportement à partir de notre connaissance. Le formalisme de la philosophie pratique de Kant n'est donc pas identique à la spécification formelle que nous soulignons ici. Chez Kant, le formalisme est le rapport de la subjectivité à l'universel, rapport qui s'exprime à travers le «par devoir» qui fonde toute morale. Nous pensons, quant à nous, que la forme exprime le rapport essentiel de la moralité à la subjectivité; c'est une relation de spécification 
formelle puisque l'action ne peut être considérée comme morale si elle n'est pas essentiellement imprégnée de subjectivité. En définitive:

La forme morale de l'action se situe dans le fait qu'elle soit mienne.

Pour Kant, le formalisme fonde le devoir et par lui toute la morale. Nous nous séparons de Kant en affirmant que cette forme n'est pas toute la morale, mais seulement une de ses dimensions.

Voilà pourquoi, en reconnaissant l'ambiguïté de la notion de morale, en affïmant sa «bi-dimensionalité», il ne paraît pas nécessaire d'en appeler au formalisme kantien pour échapper au subjectivisme pur. La morale est subjective, certes, c'est même sa spécification formelle, mais elle n'est pas entièrement subjective. Elle est formelle, certes, mais elle n'est pas exclusivement formelle. Nous affirmons que la forme de la morale est la subjectivité essentielle de l'action, mais nous affirmons également que cette spécification formelle n'est pas toute la morale.

Voyons tout d'abord les implications de l'affirmation de cette subjectivité nécessaire de l'action morale pour comprendre la condition humaine. Cette analyse permettra de construire l'anthropologie philosophique qui résulte de la prise de conscience de la morale. En effet, notre affirmation doit pouvoir révéler les conditions humaines de l'action et conduire à une compréhension particulière de l'homme. Nous n'essayons pas, ici, de comprendre le phénomène moral à partir d'une connaissance de l'homme, mais au contraire d'élaborer une nouvelle vision de la "condition humaine" à partir de la conscience morale.

Les démarches successives de cette prise de conscience sont au nombre de quatre: la signification humaine de la subjectivité morale; la révélation de la liberté; l'intention et la finalité de l'action; la responsabilité. Nous finirons cette présentation en analysant ce que nous appelons le "fait» de la morale.

\section{Signification humaine de la subjectivité morale}

L'anthropologie philosophique que nous tentons de construire par la réflexion morale, fondée sur l'affirmation de la subjectivité essentielle de l'action morale, nous oblige à discuter et à critiquer tout d'abord une conception traditionnelle, d'origine aristotélicienne, qui s'appuie, dans ses affirmations et ses conclusions, sur une connaissance préalable de la nature de l'homme. 
Aristote, cherchant à déterminer l'acte propre de l'homme, le distingue des autres activités qui sont également partagées par les plantes et les animaux. «La vie est commune à l'homme et aux plantes; or nous cherchons ce qui le caractérise spécialement. Il faut donc mettre à part la nutrition et la croissance. Viendrait ensuite la vie des sensations, mais bien sûr celleci appartient également au cheval, au boeuf et à tout être animé. Reste donc la vie active propre à l'être doué de raison». L'action propre de l'homme sera donc la vie rationnelle.

Mais Aristote remarque aussitôt que cette vie active propre à l'être doué de raison est double; il faut y distinguer deux parties: «l'une obéissant, pour ainsi dire, à la raison, l'autre possédant la raison et s'employant à penser". Il est donc préférable de considérer l'action humaine quand elle est pleinement épanouie, au moment où elle se présente comme supérieure en tous points aux autres formes d'activité. Ainsi, comme il est possible de distinguer un quelconque joueur de cithare d'un joueur accompli qui maîtrise son instrument à la perfection, il faut supposer «que le propre de l'homme est un certain genre de vie, que ce genre de vie est l'activité de l'âme accompagnée d'actions raisonnables, et que chez l'homme accompli tout se fait selon le Bien et le Beau, chacun de ses actes s'exécutant à la perfection selon la vertu qui lui est propre. A ces conditions, le bien propre à l'homme est l'activité de l'âme en conformité avec la vertu». ${ }^{10}$

Aristote n'introduit pas, dans la définition de l'action morale qui est le propre de l'homme, la double dimension que nous avons soulignée, et ne fait pas de distinction entre un jugement de fait, qui établit formellement ce qui ressortit à l'ordre moral, et le jugement de valeur par lequel ces actions sont jugées en fonction du bien et du beau. Aristote conjugue ces deux jugements dans la seule définition de l'action humaine pleinement épanouie, tout en établissant une distinction entre la vie rationnelle «qui obéit à la raison» (reconnaissance du fait de la raison), et la vie rationnelle «qui possède la raison et s'emploie à penser» (reconnaissance d'une supériorité préférentielle, ou d'une valeur). Il faut voir dans cette distinction une certaine reconnaissance de l'ambiguité de la morale.

Mais la théorie aristotélicienne doit être prise dans une perspective dynamique, l'être n'atteignant sa pleine nature que dans son enthéléchie, c'est-à-dire quand toutes les potentialités de son essence sont pleinement épanouies. Dans ce sens, il est certain que le propre de la nature humaine pleinement épanouie est d'agir selon le bien et le beau en conformité avec la vertu, enracinant la morale dans les données fondamentales de la métaphysique. Mais nous croyons respecter davantage la réalité en distinguant l'action simplement rationnelle (propre à l'homme) et l'action parfaitement vertueuse (l'idéal du sage). Or si nous voulions construire une anthropolo-

10 Aristote, Ethique de Nicomaque, Livre I, Chap. 7, 12-13 
gie à partir de la morale d'Aristote, sans recourir à la métaphysique qui la soutient (chose totalement impossible pour Aristote lui-même, mais concevable dans une philosophie post-kantienne), nous devrions logiquement conclure que l'homme agit d'abord rationnellement, et en ce sens il n'est pas a-moral, et qu'ensuite seulement il lui est possible d'agir en conformité avec la vertu. L'éthique aristotélicienne ne rejette donc pas en principe la bi-dimensionalité de la morale. Nous pensons cependant qu'en intégrantl'apport du chistianisme et de Kant nous devons faire un pas de plus et penser que l'action qui constitue l'objet propre de la réflexion morale n'est pas seulement l'action rationnelle, mais l'action empreinte de subjectivité. Le «fait moral» est plus spécifique de l'individualité que la simple rationalité.

Thomas d'Aquin reprendra l'argument d'Aristote en distinguant les actions qui ressortissent proprement à la morale, les actes humains, actus humani, des actes de l'homme, actus hominis. "Seuls les premiers sont authentiquement des actes moraux au sens où nous l'entendons, les autres, les actes de l'homme, n'étant que les manifestations de l'activité commune à tous les êtres vivants (nutrition; mouvements irréfléchis, réactions vitales spontanées, etc.). Ces derniers en effet ne sont pas à proprement parler des actions humaines, cum non sint hominis inquantum est homo. Thomas d'Aquin comme Aristote rattachent la spécification de l'action morale à la nature de l'homme affirmée préalablement. Les actions morales sont celles qui ressortissent à l'humanité en l'homme, et non à son animalité.

Nous devons toutefois souligner que la distinction proposée par la philosophie aristotelefco-thomiste entre ce qu'il y a de arationnel-humain* et ce qu'il y a d'animal en l'homme ne doit pas être interprétée à la lumière de l'anthropologie cartésienne qui établit une séparation radicale entre l'âme et le corps, entre la pensée et l'étendue. Une interprétation en ces termes de la rationalité aristotélicienne retrancherait de l'action humaine propre tout ce qui ressortit a la physiologie strictement humaine, et situerait ainsi la moralité, et par elle la liberté, dans les seules manifestations d'un esprit désincarné. L'homme, aux yeux de Descartes, demeure un composế, une dualité entre l'espace et l'étendue, quelque intime que puisse être la soudure psycho-physique garantie par la eglande pinéales. Mais justement, que deviendrât la moralité elle-même devant une simple déficience de cette glandê, le corps étant réduit à une pure animalité, simple étendue sans âme, inutile et incompréhensible matière humaine.

La philosophie contemporaine n'a pas à s'embarrasser de telles hypothèses, car les connaissances actuelles de la biologie et de la physiologie humaines apportent des arguments irréfutables contre les interprétations qui ne verraient dans le corps humain qu'une simple animalité dépourvue de toute dimension morale. II est vrai que l'homme se distingue de l'animal

11 Thomas d'Aquin, Somme théologique, la llae, q. 1, art. 1, 
dans sa propre physiologie, en raison tout d'abord du nombre plus élevé des cellules de son cerveau et des lois de complexification qui les régissent. ${ }^{12}$ C'est d'ailleurs en s'appuyant sur une connaissance de cette spécificité de la physiologie humaine que les théories déterministes du comportement, comme le «Behaviourism» de Watson, par exemple, fondent leur principes d'analyse et d'intervention sur les conduites spécifiquement humaines. Il n'est donc pas nécessaire de faire intervenir la notion d'une âme rationnelle et spirituelle pour admettre que la conduite humaine se distingue essentiellement de la conduite animale.

Il est donc possible, sans recourir à la métaphysique et sans faire appel à l'existence d'une âme spirituelle, en s'appuyant uniquement sur les connaissances du cerveau humain et de sa complexité, de montrer que I'homme peut avoir une activité typiquement humaine, in quantum est homo. Une morale biologique purement scientifique peut être fondée qui, non seulement ne contredira pas la morale spiritualiste, mais pourra même apporter certaines confirmations de ses thèses. ${ }^{13}$

Le raisonnement de cette morale est toutefois semblable à celui par lequel Aristote construisait sa morale sur des fondements métaphysiques et la notion d'une nature humaine. Mais chaque fois que l'on voudra définir l'action morale en se fondant sur la nature de l'homme, le jugement qui porte sur ce qui est moral conduit automatiquement à glisser vers un jugement de valeur sur ce qui doit être, également fondé sur la nature de l'homme. «La nouvelle biologie humaine n'est plus seulement descriptive, mais tend à être normative, c'est-à-dire à définir dans toutes les conduites humaines ce qui est humain, conforme à la nature psycho-biologique de l'être humain, ou plutôt ce qui est humanisant, va dans le sens de l'épanouissement des possibilités de cette nature, et ce qui est inhumain, déshumanisant». ${ }^{14}$ Ainsi toutes les morales qui veulent définir l'action morale à partir de la métaphysique ou de la nature humaine en arrivent à confondre le descriptif et le normatif et dissolvent la compréhension de ce qui ressortit à l'ordre moral dans les catégories objectives du normal, du naturel et du tolérable. Il en est de même avec la grande majorité des observations et analyses sociopsychologiques des comportements humains qui, partant de l'acceptation du conditionnement social comme d'une donnée de nature, contribuent à donner une image «factuelle» de la moralité, refusant de porter des jugements de valeur sur ce qui se fait, puisque que cela représente la nature humaine..$^{\text {is }}$

13 Un exemple est donné par le livre de Paul Chauchard, Biologie et morale

14 Paul Chauchard, op. cit., p. 22

15 Le livre de Gilles Lipovetsky présenté dans le Chapitre précédent, Le crépuscule du devoir, illustre cette approche. Dans une interview dans L'évenement du jeudi du 3 décembre 1992, Lipovetsky explique d'ailleurs sa position en répondant un l'un de ses critiques: «la vérité ne peut pas être une mauvaise action..». En d'autres mots, les comportements humains observables, étant humains, ne peuvent être jugés mauvais sans condamner l'homme. Alors? 
Pour Aristote cependant, et pour tous ceux qui demeurent fidèles à son approche, l'action humaine souhaitable demeure l'action de celui qui a accompli sa perfection. Le modèle n'est pas le citharède, mais le citharède accompli qui joue bien. De même, pour la biologie contemporaine, ce qui est humain est ce qui est humanisant, ce qui va dans le sens de l'épanouissement de la nature humaine. ${ }^{16}$ Nous dirons également que l'éthique de la prospérité reprend certains aspects de cette idée en suggérant que ce qui est humain est ce qui fait croître le bien-être de l'homme, ce qui augmente son bien-être matériel.

Ceci nous semble être la logique dans laquelle une conception unidimensionnelle de la morale enferme le raisonnement. Nous évitons ce piège si nous insistons sur les distinctions établies plus haut entre l'a-moral et l'im-moral, entre ce qui n'est pas humain, $a$-humain, de ce qui se fait contre l'homme, in-humain. Ce n'est donc pas à partir d'une définition de la nature humaine que nous pourrons déterminer avec sûreté la caractéristique formelle par laquelle nous pouvons savoir qu'une action est d'ordre moral, donc non a-morale.

Nous trouvons chez Thomas d'Aquin un complément à la simple notion de nature humaine qui nous paraît se rapprocher de ce que nous cherchons à établir. Les actions proprement humaines, dit-il, sont celles dont l'homme est le maître: solae actiones vocantur propiae humanae, quarum homo est dominus. ${ }^{17}$ Il est donc nécessaire de voir comment l'homme devient le maître de ses actions. Cette maîtrise, précise Thomas d'Aquin, est assurée per rationem et voluntatem, par la raison et la volonté. Or, dans la conception thomiste, raison et volonté sont les conditions propres de la liberté, et ce qui est dit de cet agent doué de raison et de volonté rejoint, «dans une certaine mesure», ce que nous appelons l'affirmation du sujet.

Nous soulignons, «dans une certaine mesure» et nous devons indiquer pourquoi.

Il est certain que, dans la conception aristotélico-thomiste, le rationnel est justement ce par quoi l' homme dépasse sa propre nature et devient sujet, en particulier quand il exprime cette rationalité par sa volonté. Dans cette ligne de pensée nous pourrions donc dire que volonté et rationalité sont justement les caractéristiques de la subjectivité. Mais nous devons aussi admettre que, dans la mesure où la subjectivité est caractérisée par des notions, telles que rationalité et volonté, nous tombons dans une contradic-

16 La téléologie d'un Teilhard de Chardin est typique de cette approche. Voir: Le phénomène humain

17 Sum. Theol., loc. cit. 
tion fondamentale, ces caractéristiques étant nécessairement communes à toutes les subjectivités; il devient donc impossible d'échapper à la position essentialiste. Une action morale qui aurait pour caractéristique d'être maîtrisée par la raison et la volonté est une action décrite et comprise en termes abstraits, en concepts nécessairement objectifs, donc universalisables. Nous croyons au contraire que l'action est morale parce qu'elle est mienne, parce qu'elle est exclusive et absolument individuelle.

Tous les êtres humains sont des sujets, mais «il n'y a que moi qui suis moi», et c'est dans la mesure où mon action est l'occasion d'une telle prise de conscience et une manifestation du moi qu'elle est authentiquement morale. C'est pourquoi il faut distinguer le moi, porteur des caractéristiques que sont la rationalité et la volonté, et l'être même du moi qui est une subjectivité irréductible à aucune nature partagée. Expliquer l'action morale par une maitrise de l'homme sur ses actions à travers la raison et la volonté revient encore, croyons-nous, à rendre compte de la morale par la métaphysique. Voilà pourquoi raison et volonté ne rendent pas compte à nos yeux de tout ce que le recours à la subjectivité implique. Car il n'y a pas d'autre fondement de la subjectivité en morale que l'acte par lequel je l'affirme.

\section{Mon action est morale non pas parce que je suis homme,} mais parce qu'elle est mienne.

Max Scheler exprime cette même idée en disant: «L'acte n'est jamais objet». ${ }^{18}$ Doit-on dès lors craindre qu'une telle affirmation conduise au subjectivisme moral absolu? Il faudrait certes répondre par l'affïmative si la morale était unidimensionnelle, si la forme de la morale était toute la morale. Mais la révélation de la subjectivité dans l'action morale n'en est que la dimension formelle.

Toutefois, affirmer que l'action doit être mienne pour ne pas se fondre dans l'amoralisme n'explique par encore tout. La subjectivité nécessaire à l'acte moral permet de découvrir que l'homme ne peut être compris que dans l'action et par lui-même, dans son engagement et sa liberté. S'il existe une morale, dès lors, c'est qu'elle est construite par l'intervention du sujet dans chacun des actes qu'il assume. La morale n'est pas donnée dans une nature préexistant au sujet; c'est lui qui la construit comme être libre et responsable.

18 Max Scheler, Le formalisme en éthique, op. cit., p. 380 


\section{La révélation de la liberté}

Si l'action doit être subjective pour être morale, elle doit se distinguer essentiellement de ces «actions de l'homme» en tant qu'homme, celles qui sont soumises aux déterminations d'une nature universelle et objective présente en chaque individu, mais qui ne constituent pas sa propre identité. En raison de la présence de cette nature, l'homme qui croit parfois marquer sincèrement l'action par sa subjectivité s'illusionne souvent par méconnaissance de sa nature et de ses possibilités. C'est un autre qui agit en moi, I'homme universel qui se manifeste, tant dans mes faiblesses que dans ma vertu, mais ce n'est pas réeellement moi. Cet homme universel agit parfois contre moi. L'illusion consiste à croire que la subjectivité se découvre spontanément, en surface, à un niveau que l'on pourrait qualifier d'épidermique. Le moi authentique, lui, se cache dans les profondeurs de l'être. Le vrai sujet, en effet, le vrai moi, ne peut pas être apparent, saisissable directement. L'homme est toujours plus intérieur et plus profond qu'il ne le pense et ce qu'il prend pour une manifestation de lui-même n'est souvent qu'une manifestation de sa structure.

La connaissance objective et scientifique de l'homme, en réduisant à l'objectivité a-morale une foule de conduites que l'homme risque de prendre pour son action propre, fait perdre ses illusions à l'esprit. Elle oblige l'homme à rechercher plus loin, plus profondément et plus intérieurement cette réelle et authentique subjectivité par laquelle il pénètre dans le domaine moral véritable. C'est pourquoi il faut souligner que l'étude du comportement «naturellement» humain contribue à élargir la connaissance du domaine de l'a-moral, et permet ainsi à l'homme à s'écarter toujours plus de ces activités qui procèdent par lui mais qui ne sont pas de lui. Il progresse ainsi sur le chemin qui le rapproche de lui-même.

Ainsi, en enfermant dans l'ordre de l'a-moral les illusions de la subjectivité, la connaissance objective de la nature humaine peut préparer une prise de conscience du moi plus pure et plus authentique. Si je reconnais, en moi, ce qui n'est pas réellement moi, je serais mieux préparé à découvrir ce qui est totalement moi, et l'assumer.

C'est pourquoi, en situant le sujet en dehors et au delà de tout ce que l'objectivité a-morale détermine, la connaissance de la nature humaine peut permettre à l'homme de prendre conscience de sa liberte..$^{19}$ Cette prise de conscience est directement liée à l'affirmation de la subjectivité essentielle de l'action morale.

${ }^{19}$ La psychanalyse ne fait pas autre chose quand elle vise à libérer le sujet du conditionnement de son comportement par l'inconscient. 
En effet, la philosophie pratique peut estre révélatrice de la liberte por le même acte de reflexion qui permet au sujet, dans l'action, de prendre conscience de lui-même. Il n'est dès lors pas possible de décrire cette liberté par des concepts. En d'autres mots, comme cette liberté se decouvre par le sujet, elle demeure au-delà de toute détermination, elle est indescriptible. La décrire, en effet, serait l'identifier à une réalité du monde objectif, done la nier.

Placée ainsi au delà de toute détermination, la libertế ne peut apparaître, au niveau de l'analyse conceptuelle, qu'à travers une démarche négative. Comme la subjectivité, la liberté qui est révélée par l'analyse de la négation par suppression de sens, "moral - a-moral», se présente tout d'abord comme formelle. En effet, si l'a-moral est ce qui est soumis aux déterminations de l'objectivité et des lois de la causalité, il faut admettre que le moral échappe à ces conditions, qu'il n'est aucunement soumis à ces déterminismes, qu'il est donc libre.

Dès lors, parler d'action morale ou parler d'action libre, parler d'action libre ou parler d'action individuelle et subjective, c'est utiliser des termes différents pour décrire la même réalité morale. Si l'action morale est libre, il faut affirmer aussi que le sujet lui-même est libre, puisque l'action morale est la manifestation la plus authentique de ce que le sujet est en lui-même.

Certes, on pourra objecter que nous renversons ici les apparences du réel et les exigences de la logique et que nous considérons comme conséquence ce qui semble en fait un antécédent. Ne devrions-nous pas, en effet, nous demander si notre action est morale parce qu'elle est libre, ou si nous sommes libres parce que notre action est morale. Notre liberté se déduit-elle de la morale, ou n'est-ce pas plutôt la morale qui doit être déduite de notre liberté?

Il y a deux manières d'aborder le problème. La liberté, en effet, peut être considérée soit du point de vue ontologique ou métaphysique, soit d'un point de vue purement moral et pratique.

Si nous nous plaçons dans une perspective ontologique, considérant l'être premier par rapport à l'agir, l'action ne peut être dite libre que dans la mesure où l'être lui-même se sera révélé libre. C'est dans ce cas la liberté qui fonde la morale. Elle la fonde ontologiquement, dans l'être de l'agent; elle est vraiment la ratio essendi de la morale. Mais, dans ce cas, il faut rechercher les caractéristiques et les principes de la morale dans le domaine de la métaphysique, et non en elle-même. La liberté devient alors une essence, une nature commune à tous les hommes. Peut-elle dès lors caractériser le sujet? Il faudrait alors rechercher l'essence de la liberté dans les déterminations de la nature et il faudrait la prouver. Mais la liberté ne serait- 
elle pas déterminée par cette preuve même? Est-il raisonnable et rationnel de parler d'une liberté déterminée?

Nous croyons que la perspective ontologique oriente la recherche de la liberté dans une voie qui conduit à des contradictions conceptuelles inévitables. Certes, cette perspective efface une apparente contradiction de départ qui consistait à intervertir le conséquent et l'antécédent. Nous affirmons cependant que cette logique conduit à une impasse et qu'une recherche exclusivement morale de la liberté est plus conforme aux pouvoirs et aux limites de notre raison.

La liberté, croyons-nous, ne se manifeste pas dans une connaissance objective de l'être, ni même dans une prise de conscience immédiate de soi, comme l'existence dans le cogito cartésien, mais à travers le dédale de ses manifestations multiples, parfois dans l'incohérence. Cette découverte progressive de la liberté est plus respectueuse du sujet, car elle ne s'impose pas à lui comme une donnée immédiate, mais grandit avec lui à travers l'expérience de l'action. Découverte de la liberté et prise de conscience de soi par l'action morale deviennent ainsi concomitantes. La métaphysique nous propose une liberté préétablie, donnée une fois pour toutes, à laquelle nous sommes «condamnés», pour reprendre l'expression sartrienne. Mais la poursuite d'une révélation de la liberté par la recherche morale permet de reconnaître, dans la difficulté même de la révélation de la liberté, la possibilité d'une conquête de soi. Ainsi la liberté devient plus intime au sujet et plus profonde, car elle apparaît comme le résultat de notre propre effort. La morale nous propose ainsi une liberté à conquérir, une liberté "comme devoir», et nous invite à la construire. Nous ne sommes pas nés libres; nous avons l'obligation de le devenir.

La morale devient donc la ratio cognoscendi de la liberté. Elle est à l'origine de la prise de conscience d'une liberté possible. La morale ne prouve pas la liberté, elle la postule, elle la requiert, l'exige; en d'autres termes, elle la présente comme une obligation. Puisque l'action n'est morale que par le sujet, ce dernier «doit» être libre. Nous pourrions ajouter: s'il y a une obligation morale, ce n'est pas «parce que» nous sommes libres, mais «pour» être libres.

Jusqu'ici, la liberté que nous révèle l'analyse conceptuelle du «moral» conserve un caractère formel, car sa connaissance est issue d'une négation par suppression de sens. Elle demeurera telle tant qu'elle ne sera pas intégrée par l'expérience dans une vision plus globale de l'homme que nous donnera une anthropologie reconstruite autour de l'action morale. Mais le chemin par lequel elle se révèle, justement parce que cette liberté n'est pas une donnée immédiate qui s'impose, mais une possibilité qui exige, laisse entrevoir une réalité pleine de dynamisme et d'enthousiasme, et à la limite porteuse d'un authentique pouvoir d'agir. Nous dirons que c'est justement parce qu'elle est morale que notre action peut être dite à la fois libre et porteuse de libération. 


\section{Intention et finalité}

Si la subjectivité de l'action morale postule la liberté, elle demande également que les notions d'intention et de finalité soient comprises dans l'analyse de l'action en tant que morale. Une action libre, en effet, libre en raison de l'absence de toute détermination qui ne soit pas issue du sujet luimême, ne peut recevoir de spécificité propre que par une intention du sujet. Nous dirons que l'intention est la libre détermination de l'action par le sujet. Elle est l'acte par lequel le sujet détermine la fin de l'action.

Cette intention n'est donc pas une spontanéité pure, ni la simple manifestation d'une capacité à agir. Une spontanéité pure, en effet, ne représenterait qu'un jaillissement désordonné, un éparpillement des potentialités du sujet, une manifestation non-réfléchie et non maîtrisée de ses puissances et de ses inclinations, une expansion cahotique de son être dans l'objectivité, l'expression désordonnée d'une nature dans laquelle le sujet se perd. En reprenant ici les termes de Thomas d'Aquin, nous dirons que l'action purement spontanée n'est pas morale, parce que non maîtrisée.

Pour être moral, le pouvoir spontané d'agir doit être maîtrisé par le sujet, il doit être voulu, il doit tendre vers une détermination que lui impose le sujet. René Le Senne le dit en ces termes: «ne relève de la morale que l'action en vue d'une détermination $\gg .^{20}$ Le sujet moral n'est donc pas seulement le centre d'une spontanéité agissante, mais la source de l'orientation de l'action vers une fin.

Mais, comme nous l'avons vu dans l'analyse historique, ce n'est pas la finalité à elle seule qui fonde la morale. Tous les appétits qui sont à l'origine de nos conduites spontanées visent une fin qui leur est propre, cela est évident, mais le déterminisme de l'instinct qui les caractérise est justement ce que la morale rejette, car il nie la liberté et la possibilité de la morale ellemême. Kant a fait remarquer que si la moralité consistait uniquement à poursuivre certains buts et à réaliser certaines fins, la nature aurait mieux fait de situer dans l'instinct lui-même la force de nous y conduire automatiquement. De même, si le thomisme reconnaît que la volonté doit nécessairement vouloir le bien, il souligne, à la suite d'Aristote, que la volonté ne visera pas le bien tel qu'il est donné en lui-même, mais tel qu'il est présent à la conscience, tel qu'il est connu, bonum apprehensum. La finalité morale n'est pas inscrite dans les choses, elle est avant tout reconstituée par l'intention consciente, et reconstruite par la rationalité de l'homme.

L'intention implique donc connaissance et conscience, une intervention du sujet qui fait que la détermination de l'action ne provient pas d'un objet, d'une nature ou d'une fin indépendante du sujet. Plaçant cette nécessité dans

20 René Le Senne, Traité de morale générale, p. 4 
sa conception de la personne, Max Scheler écrit: «ce qui appartient à l'essence de la personne, c'est de n'exister et de vivre qu'en effectuant des actes de visée intentionnelle. Par définition même, elle ne peut donc être «objet». ${ }^{21}$

Cela veut dire que, pour agir moralement, il ne suffit pas que le sujet veuille son propre vouloir, ce qui reviendrait à abandonner l'action à une spontanéité pure sans finalité, mais il doit toujours vouloir quelque chose. Toutefois ce quelque chose doit être librement voulu. L'action morale est donc une action «intentionnée», une action nourrie d'une visée libre par laquelle le sujet se porte vers un but. Il n'y a pas d'acte gratuit ${ }^{22}$, il y a toujours une finalité.

C'est pourquoi, dans la perspective où nous avons situé cette analyse, nous dirons que l'action morale n'est pas l'action accomplie, terminée, une action close qui aurait déjà épuisé sa visée, mais qu'elle est essentiellement à faire, celle qui ne se comprend que par ce qui doit être, celle qui implique fondamentalement une obligation. L'action morale ne peut pas être découverte dans ce qui est, ou être contemplée dans ce qui est accompli, elle est toujours à construire.

D'autre part, l'intention morale n'est pas de la nature de l'intentionalité du connaître; elle ne vise pas la possession de son objet, mais la création de son but. L'objet de l'intention morale n'est pas donné, il est à faire, il est à conquérir. Il n'y a pas de morale si le sujet renonce à sa visée, à la création par laquelle il est le seul à pouvoir donner un sens et une valeur à ce qui n'est pas encore mais demande à être. Cette exigence formelle de la morale nous fait comprendre que le contenu matériel de cette intention ne peut se présenter que comme une obligation, que comme une réalité à construire.

21 Max Scheler, op. cit., p. 395

22 Un acte gratuit, comme on l'entend généralement, est un acte qui n'a pas d'autre but que l'acte lui-même. Mais ce n'est pas parce que l'acte est sa propre fin qu'il n'a pas de fin. Si c'était le cas, il ne pourrait y avoir d'intention, et l'acte gratuit ne serait donc pas autre chose qu'un acte inconscient ou involontaire. Or l'acte gratuit est justement volontaire; le sujet a l'intention de le commettre. Nous voyons donc que l'être humain ne peut s'empêcher, dans un acte libre, de vouloir quelque chose, même si c'est son propre vouloir. C'est dans cette perspective que nous voyons l'aspect négateur et dégradant de l'égoïsme. En s'intentionnant lui-même, l'homme devient un objet pour lui-même; il nie par là sa liberté et son être en tant que personne. 


\section{La responsabilité}

La dernière implication de la dimension subjective de l'action morale est la responsabilité. En effet, si l'action est libre et intentionnée, si le sujet en est la seule source et lui donne toutes ses caractéristiques, il doit en être reconnu pleinement responsable.

Etre responsable signifie ici que le sujet, dans l'action qui lui est propre, se manifeste, s'engage, se livre en tant que sujet, sort de lui-même et accepte de dévoiler l'intention cachée au fond de son intimité. Il publie les fins qu'il poursuit et témoigne de lui-même en se confondant avec ce qu'il fait. «Je suis moi dans la seule mesure où je suis responsablem. ${ }^{23}$

Du point de vue moral, la responsabilité est premièrement la manifestation du sujet au monde, l'ouverture du sujet à la communication avec autrui. Ainsi l'action morale n'est pas seulement l'occasion de révéler le sujet à lui-même, mais aussi de le révéler à autrui. Accepter sa responsabilité, c'est communiquer ses intentions aux autres, c'est assumer un rôle qui prend une dimension sociale, c'est une façon de ne plus être seul. Ainsi, par la responsabilité, la morale échappe au subjectivisme.

Qu'est-ce que cela signifie? Introduire la notion de responsabilité dans la définition de l'action morale, c'est reconnaître qu'il n'est pas possible de porter un jugement sur cette action - non pas un jugement de fait, mais un jugement de valeur - sans reconnaître en elle le sujet qui l'a commise, dans ce qu'il a de plus intime et de plus secret. L'action morale devient donc l'occasion d'une réciprocité des consciences qui fait que la prise de conscience de soi, dans l'action, représente également une ouverture et un don de soi à l'autre. En affirmant ma responsabilité, j'admets que l'intimité de mon propre sujet soit partagée; je manifeste l'existence de ma personne dans ce que je fais. Mais ce même engagement consiste à reconnaître, dans l'action responsable d'autrui, une manifestation de subjectivité semblable à la mienne. Je découvre alors une autre liberté unique, je reconnais le sujet d'autrui comme sujet, je le respecte. Et comme ce respect représente luimême une action dont je suis responsable, le respect d'autrui devient une action morale qui me révèle à moi-même. Cet échange mutuel qui se produit dans le respect de la responsabilité de l'autre est au coeur du fondement de toute morale sociale.

En effet, puisque, pour être morale, l'action doit être responsable, nous dirons qu'elle ne peut être morale sans être sociale. Elle exige du sujet, non seulement qu'il tienne compte de la communauté des sujets, mais qu'il s'y engage lui-même. La responsabilité devient ainsi une responsabilité «en-

Emmanuel Levinas, Ethique et infini, p. 97 
vers les autres». Je suis responsable de mon action non seulement à mes propres yeux, mais à la face du monde, et cette responsabilité exige que mon action ne doit pas trahir la confiance et le respect qu'autrui manifeste pour ce que je lui propose comme mien.

La responsabilité est toujours sociale; elle est un engagement.

Dans l'action morale, le sujet n'est jamais seul. C'est pourquoi la responsabilité ne doit pas être confondue avec la sincérité. La sincérité, qui exprime la correspondance aussi étroite que possible de l'état de conscience du sujet avec son action, n'exprime en fait que cette seule correspondance. Elle ne qualifie l'action qu'en fonction du seul sujet. Certes, la sincérité est nécessaire pour qu'il y ait sentiment de responsabilité, mais ce n'est pas encore suffisant, car elle ne demande pas au sujet de sortir de lui-même. Enfermé en lui-même, le sujet peut bien prétendre être sincère, mais comment distinguer alors cette sincérité de la subjectivité épidermique dont nous avons parlé plus haut, et qui se confond avec les tendances, les inclinations, les déterminations spontanées de la nature, de ce qui est en moi mais n'est pas de moi? On peut certes parler d'une sincérité avec soi-même, mais il est difficile de la distinguer d'une spontanéité incontrôlée si elle n'est pas mise à l'épreuve de l'authentique responsabilité qui engage envers autrui. Il n'y a pas de responsabilité réelle sans sincérité; mais il peut exister une sincérité irresponsable, et dans ce cas elle n'est pas morale.

Mais si nous croyons nécessaire de distinguer la responsabilité de la sincérité, cette affirmation subjective de la correspondance du sujet et de son action, il est également nécessaire de la distinguer de l'imputabilité, qui est également l'affirmation d'une correspondance entre l'action et le sujet, mais prononcée cette fois par autrui. Imputer des actions à un sujet consiste à le reconnaître comme l'auteur véritable de ses actions et à en manifester publiquement la responsabilité. L'imputabilité est une responsabilité jugée de l'extérieur. Max Scheler a souligné l'importance de cette notion qui est formée, dit-il, «du dehors, d'après les actes visibles du sujet». ${ }^{24}$ Mais confondre l'imputabilité et la responsabilité serait ignorer que la responsabilité véritable exige également la sincérité, c'est-à-dire un jugement sur l'action formulé du dedans, par le sujet lui-même. Etre pleinement responsable, finalement, implique que le sujet l'est «devant quelqu'un» (imputabilité), et «devant soi-même» (sincérité). La responsabilité morale n'est donc, ni la sincérité prise exclusivement, ni l'imputabilité du jugement social, mais la conjonction des ces deux notions.

24 Max Scheler, op. cit., p. 487. 
Il faut toutefois souligner que, par l'imputabilité, le jugement de responsabilité fait intervenir autrui dans le processus de révélation du sujet. En nous imputant nos actions, c'est-à-dire en affirmant publiquement notre responsabilité dans nos actes, autrui, ou la société, nous reconnaît comme les réels créateurs de nos actions, comme les seuls porteurs de nos intentions. Par l'imputabilité, autrui nous force, pour ainsi dire, à la responsabilité, et de ce fait nous conduit à la moralité. C'est une autre forme de l'appel au sujet.

Il y a en effet deux attitudes qui peuvent compromettre la reconnaissance d'une responsabilité morale, et lui substituer des «excuses». Il y a d'une part l'attitude qui consiste à excuser l'action en l'expliquant; et d'autre part celle qui consiste à l'excuser en la justifiant.

«Expliquer» un acte, c'est montrer comment le jeu de diverses nécessités, plus ou moins évidentes, a conduit l'agent à agir de telle ou telle façon. C'est ramener l'action au simple résultat de déterminations objectives, à la manifestation d'une spontanéité irrésistible, à une contrainte sociale impérieuse, un conditionnement ou un trait de caractère insurmontable. En réduisant ainsi l'action à des causes objectives «a-morales», on excuse l'agent, mais on nie en lui, en même temps, l'existence d'un sujet responsable. Il y a beaucoup de cette attitude dans la «tolérance» laxiste que manifeste une certaine libéralisation des moeurs dans nos sociétés "prospères». L'acceptation d'une "morale indolore», qui ne secoue ni ne responsabilise l'individu, explique en partie la disparition du sujet responsable dans la société contemporaine.

"Justifier» un acte, au contraire, consiste à l'excuser en faisant appel à une finalité extérieure, souvent se rattachant à un ordre supérieur de fins, l'agent n'étant considéré que comme simple instrument de celles-ci. ${ }^{25} \mathrm{C}$ 'est une finalité qui n'est pas directement «intentionnée» par le sujet, mais qui s'impose à lui, et qu'il ne peut refuser au nom d'un ordre moral, social ou politique supérieur. Tuer, dans le cas d'une guerre, n'est pas un acte imputable au soldat individuel qui agit, mais un acte dépendant de fins politiques qui le dépassent et dont il n'assume pas la responsabilité. Certes le soldat lui-même n'est pas poussé à tuer son ennemi par des inclinations naturelles et irrésistibles; donc son acte n'est pas explicable de cette façon. Mais son acte, condamnable par ailleurs, est justifié par une finalité supérieure qui s'impose à lui et dont il n'est pas personnellement responsable.

${ }^{25}$ Cette justification doit être distinguée de celle que des sujets présentent parfois pour excuser les conséquences d'actes fondés sur de bonnes intentions qui ont eu de malheureux effets. Dans ce cas, l'agent ne cherche pas à justifier son acte en en déclinant la responsabilité, mais il demande qu'on ne le juge pas simplement sur les résultats, mais aussi sur l'intention. 
Faire appel ainsi à l'explication ou à la justification, c'est éviter d'impliquer la responsabilité directe de l'agent; c'est lui retirer son caractère moral et sa dignité de sujet. L'analyse des comportements dans l'éthique de la prospérité fournit une foule d'exemples dans lesquels la responsabilité du sujet est niée, car le succès ou l'échec de son action n'est pas imputé à la personne, mais à ses compétences, à sa performance ou à l'organisation dans laquelle il travaille. Le souci d'efficacité entraîne souvent une imputation «objective», explication ou justification, qui ignore les sujets.

Mais imputer une action à un sujet particulier, faire appel à sa responsabilité pour lui reconnaître son indispensable moralité, c'est contribuer à révéler le sujet à lui-même. Comme nous avons dit plus haut que la morale était révélatrice de la liberté, nous pensons pouvoir affirmer que, par la notion de responsabilité qu'elle implique, la morale est également la ratio cognoscendi du sujet. En le privant de cette responsabilité, on empêche le sujet de prendre conscience de lui-même et de ce qui le définit en tant que sujet. Finalement, moralité et responsabilité s'identifient, pour ainsi dire, dans un sujet libre et créateur.

\section{Le fait de la morale}

L'analyse de la dimension formelle de la morale nous révèle les caractéristiques essentielles de ce que nous avons nommé «le fait de la morale», la réalité qui se distingue de ce qui n'est pas moral. Mais cela ne nous a encore rien dit du contenu ou de la «matière» de cette morale, car les notions qui se sont présentées ne pouvaient l'induire. Nous avons reconnu la donnée essentielle de ce fait de la morale dans la présence du sujet. Ceci nous permet de prendre conscience de l'homme par sa nature morale, ce qui implique un jugement de valeur fondamental, à savoir qu'il est préférable pour l'homme d'agir moralement plutôt qu'a-moralement.

Nous pouvons donc conclure. La première obligation humaine, nous dirons l'obligation fondamentale, est que son action soit morale au sens qui nous est apparu dans ces pages. En d'autres mots, nous dirons que l'amoralité est aussi a-humaine, car il n'est pas possible d'être homme sans être moral, donc sans être sujet.

Cette obligation est première et essentielle car elle exige de l'homme de se construire soi-même, en même temps qu'il établit le "fait de la morale» par la nature subjective de son action. Il en va de son existence en tant que sujet. Finalement, être moral et être soi-même sont, pourl'homme, une seule et unique obligation. Dans cette perspective nous dirons que:

l'a-moralité est im-morale. 
L'action morale étant l'action d'un sujet unique, et non pas la manifestation d'une nature objective, elle doit être premièrement libre, libre de tout, à la limite nous pourrions dire libre de l'humain. Elle ne peut en aucun cas se confondre avec les conduites humaines non maîtrisées dont la connaissance objective rend compte en faisant intervenir les déterminations causales de notre nature, ni avec l'action marquée par la rationalité universelle de l'homme.

Libre de tout ne signifie cependant pas que l'action morale puisse être indifférente, car cette liberté n'aurait pas de sens si l'action n'était pas «intentionnée», c'est-à-dire si elle ne visait pas une fin que le sujet se donne à lui-même.

Cette détermination se présente comme un devoir être, c'est-à-dire une obligation, qui ne peut aucunement trouver son efficacité dans une détermination préalable ou universelle. C'est par l'intention qu'est ainsi révélée la responsabilité de l'agent. Par cette responsabilité, il se manifeste comme sujet ouvert aux autres sujets. Tout en gardant son fondement moral dans la responsabilité subjective, l'acte peut devenir l'objet d'un jugement d'autrui, car le sujet la lui propose, pour ainsi dire, en acceptant que sa responsabilité puisse aussi être l'objet d'imputabilité.

L'action morale doit donc revêtir une dimension sociale, ou du moins une dimension intersubjective sur la base de laquelle il est possible de construire une société de personnes libres et responsables. 


\section{MORAL - IM-MORAL : La valeur morale et l'objectivité inter-subjective}

La négation par «renversement de sens», contrairement à la négation par «suppression de sens», conserve entre les termes contraires une certaine correspondance, une sorte de communauté de nature ou de référence, une spécificité par laquelle les deux termes se répondent. Un peu comme des frères ennemis: ils n'en demeurent pas moins frères au delà de leur inimitié.

Une telle catégorie de négation par renversement peut être illustrée par les notions de sympathie et d'antipathie. La sympathie témoigne d'un sentiment favorable à autrui; l'antipathie d'un sentiment défavorable. L'attraction s'oppose ainsi à la répulsion, mais dans un ordre commun, celui des sentiments. Les deux orientations s'appuient sur un fait commun, l'existence du sentiment, la capacité de pouvoir ressentir quelque chose, positivement ou négativement, dans la relation à autrui. L'absence de sentiment, c'est-à-dire la négation par suppression de ce qu'implique le sentiment, serait l'apathie, la totale indifférence.

La négation «moral - im-moral» étant de cette nature, les deux termes doivent être rattachés, tout en se contredisant, par une caractéristique commune, qui doit être de telle nature qu'elle puisse être exprimée ou perçue, positivement ou négativement selon les cas, sans disparaître. C'est pourquoi la différence qui sépare le moral de l'im-moral ne sera pas fondée sur une forme spécifique du «moral» - puisque la forme, nous l'avons vu, ne peut pas être niée sans disparaître - mais dans un contenu ou une matière, ce que nous appellerons la matière morale. La terminologie proposée par Max Scheler parle de caractéristique «matériale» ${ }^{26}$. C'est par cette matière morale que nous sommes à même de distinguer une action morale d'une action im-morale et que nous pourrons comprendre le sens du «renversement» qui se produit dans leur négation.

26 Nous ne retiendrons cependant pas la terminologie schélérienne car la notion de "material» a pour but de marquer une opposition fondamentale à Kant, et non de déterminer l'aspect matériel des valeurs. Pour Scheler, en effet, les valeurs se présentent a priori, et elles s'opposent ainsi à l'apriorisme kantien qui ne pouvait être que formel. Nous ne pensons pas, par contre, que les valeurs soient aprioriques. L'utilisation du terme de Scheler pourrait ainsi porter à confusion. Nous garderons donc l'adjectif «matériel» pour qualifier ce qui n'est pas formel. 
Nous considérons cette caractéristique comme matérielle car elle doit être neutre en elle-même pour admettre d'être prise positivement dans un cas et négativement dans l'autre. La notion de matière est prise ici dans son sens aristotélicien, c'est-à-dire comme un possible non déterminé, un possible ouvert, disponible pour sa détermination. La matière morale doit aussi être indéterminée si elle doit pouvoir devenir "morale» dans un cas, et «im-morale» dans l'autre.

Pour reprendre l'illustration proposée ci-dessus, le sentiment nous en offre un bon exemple. Le sentiment, pris en lui-même, n'est que la possibilité de la sympathie ou de l'antipathie, mais il n'est pas déterminé en luimême à être l'un plus facilement que l'autre, comme il n'est pas déterminé d'ailleurs à être autre chose que la sympathie ou l'antipathie. ${ }^{27}$ Mais si le sentiment n'est qu'un possible indéterminé, nous devons reconnaître qu'il n'existe pas concrètement, qu'il n'est pas une réalité à lui seul, qu'il n'est pas une substance à laquelle s'ajouteraient la sympathie ou l'antipathie comme qualités accidentelles. Le sentiment n'existe pas en dehors de son actualisation en sympathie ou en antipathie, mais nous pouvons le penser conceptuellement, le supposer virtuellement, indépendamment de ses actualisations possibles, car c'est par lui que se comprennent la sympathie et l'antipathie, bien qu'elles soient une négation l'une de l'autre.

De la même façon, nous dirons qu'il y a une matière morale, qui n'existe réellement que dans son actualisation en actions morales et en actions immorales, mais que nous devons penser cette matière en dehors de ses actualisations concrètes et contraires, car c'est par elle seule que le moral et l'im-moral deviennent compréhensibles l'un par rapport à l'autre dans leur négation même. Nous ne pourrons cependant appréhender cette matière morale qu'à partir des réalités par lesquelles elle nous est donnée dans l'expérience morale, c'est-à-dire à partir des actions morales et des actions im-morales.

Nous devons tenir compte que, par cette démarche, nous remontons, pour ainsi dire, des actualisations concrètes à la matière possible que ces actualisations supposent. Aussi nous ne tenterons pas, pour l' instant, d' analyser les conditions d'actualisation de la matière morale, mais simplement les voies par lesquelles elle se découvre. Nous reviendrons sur le problème de l'actualisation morale au chapitre suivant.

27 Il ne faut pas prendre cet exemple limité comme une ébauche de théorie des sentiments. Il a pour seul but d'illustrer notre objet. 


\section{Le jugement de valeur et la norme}

Lorsque nous avons analysé la notion de responsabilité, nous avons reconnu que l'action morale, au sens formel, avait un caractère social essentiel. «Social» signifie ici que l'action d'un sujet, aussi marquée qu'elle soit de subjectivité pour être formellement morale, devient pour autrui un «objet» au moment où l'action se manifeste et que, de ce fait, la responsabilité subjective se transformait en une responsabilité envers les autres. En acceptant cette responsabilité ouverte, le sujet acceptait de n'être plus le seul à pouvoir juger et comprendre son action dans le secret de sa sincérité, mais il offrait, pour ainsi dire, sa propre action au jugement d'autrui, en particulier dans le jugement d'imputabilité.

Or cette notion de jugement n'a pas été établie à ce stade car l'analyse formelle ne nous permettait pas de le faire. Nous avons simplement reconnu qu'un jugement devait avoir lieu. Il nous faut maintenant chercher quelle est la nature de ce jugement qu'autrui porte sur les actions responsables.

Il est d'abord nécessaire d'introduire quelques distinctions concernant le jugement lui-même. De façon générale, le jugement affirme l'existence d'une relation déterminée entre deux ou plusieurs termes. Sans entrer ici dans une analyse détaillée de la logique du jugement, nous dirons qu'il existe deux grandes catégories de jugements. Ceux qui affirment l'existence d'une liaison de vérité ou d'erreur, que nous qualifions de jugements de fait (la neige est blanche), et ceux qui affirment une relation de hiérarchie, de classement ou de préférence, qui peuvent soit confirmer l'existence d'une hiérarchie ou de classement d'ordre quantitatif (le cerveau humain est plus complexe que le cerveau animal), ou une hiérarchie ou classement d'ordre qualitatif (je préfère le vin à l'eau; respecter la vie est préférable à sa destruction). Mais il faut distinguer encore, parmi les jugements de préférence dans l'ordre qualitatif, les jugements d'estimation et les jugements de valeur. Les uns et les autres affirment des préférences, mais le jugement d'estimation affirme une préférence de réalité, de fait, (je préfère le vin à l'eau) et exprime, selon Durkheim à qui nous empruntons cette distinction, «des états déterminés du sujet» ${ }^{28}$; ce jugement peut donc être assimilable aux jugements de réalité. Par contre, le jugement qui nous apparaît comme l'authentique jugement de valeur affirme une relation hiérarchique ou de préférence avec le désirable, non pas tel qu'il est nécessairement préféré par un ou des sujets dans leur état particulier, mais tel qu'il apparaît dans le possible, en d'autres mots dans l'idéal (respecter la vie est préférable à sa destruction).

28 Emile Durkheim, «Jugements de valeur et jugements de réalité», Communi cation, Atti del IV Congresso Internationale di Filosofia, 1911, Vol. I, p. 99 
Le jugement de valeur n'affirme donc pas une réalité, comme la préférence qu'affirme le jugement d'estimation, mais une possibilité souhaitable qui ne contient pas en elle-même la garantie de son actualisation. Il est «vrai» que je préfère le vin à l'eau, c'est un fait indéniable puisque je l'affirme. Mais ce n'est pas en affirmant que le respect de la vie est préférable à sa destruction que cette préférence se manifeste comme une réalité; c'est de l'ordre du souhaitable.

Il y a donc une différence entre le jugement de préférence factuelle, qui révèle un état du sujet, et un jugement de préférence de valeur, qui affirme la désirabilité d'une relation concernant l'idéal, entre le jugement qui porte sur ce qui est, et celui qui affirme ce qui devrait être.

De quelle nature sont dès lors les jugements qui portent sur l'action morale? Ils sont de deux ordres: ceux que le sujet lui-même porte sur sa propre action; et ceux qu'autrui porte extérieurement sur l'action du sujet.

Quand le sujet agit, il juge lui-même son action du point de vue de sa propre sincérité et responsabilité, et la propose comme authentique en affirmant: "cette action est mienne et j'en suis responsable». C'est un jugement que nous appelons «d'estimation subjective», qui affirme la relation d'authenticité entre le sujet lui-même et son action. Ce n'est pas un jugement de vérité; le sujet ne dit pas: "cette action est vraie», car cela n'aurait pas de sens, mais il marque une préférence: «cette action est mienne», par laquelle il affirme la supériorité de ses actions propres sur les conduites qui «sortent» de lui mais ne sont pas réellement de lui, et dont il n'est pas responsable. Or ce jugement est propre au sujet, et ne peut être porté que par lui seul. Il se différencie en fait de la déclaration d'imputabilité dont nous avons parlé plus haut qui, elle, ne peut être un véritable jugement d'estimation subjective, mais un postulat sur la responsabilité du sujet, afin de permettre à la société de juger le sujet à travers son action. Mais nous sommes encore, par ce jugement, au niveau de la reconnaissance de la dimension formelle de la morale.

Le jugement social, lui, considère l'action non pas en raison de sa forme subjective mais en raison de son contenu. Par ce jugement, autrui, ou le groupe social, approuve ou désapprouve, loue ou condamne, élit ou rejette l'action dont il est le témoin. Dans ce jugement, la société dit qu'il est louable d'agir de telle sorte et condamnable de le faire de telle autre manière, sans tenir compte du sujet qui en est l'auteur. L'action se trouve alors détachée de son lien avec la subjectivité de l'agent et devient un «objet» de jugement; elle est alors évaluée en raison de ce qu'elle représente en ellemême, de son contenu, de sa matérialité.

$\mathrm{Ce}$ jugement est un véritable jugement de valeur, car il affirme une préférence dans une relation positive ou négative avec ce qui est considéré comme désirable. Or c'est la possibilité d'en faire un jugement positif ou négatif qui constitue la matérialité de la valeur. En effet, si je dis: «je préfère 
le vin à l'eau», j'affirme une réalité qui ne peut pas faire l'objet d'un jugement si cette préférence n'est pas réelle. Par contre, quand j'affirme que «le respect de la vie est préférable à sa destruction», je maintiens ce jugement même si l' acte que je juge avec ce critère nie une telle préférence. Je peux donc juger un assassinat par un jugement de valeur de préférence, et continuer à dire que le respect de la vie est préférable à sa destruction, même si la réalité de l'action particulière représente une négation de la valeur affirmée. L'action morale est alors jugée en fonction d'un idéal, d'un contenu de valeur désirable par rapport au contenu concret de l'action ellemême. Nous appelons norme ce contenu moral idéal qui exprime la valeur positive préférée au nom de laquelle le jugement moral de valeur peut être prononcé.

Le jugement de valeur porté sur une action concrète ne consiste pas à expliquer cette action en fonction des déterminations qui peuvent en rendre raison, ni à la déclarer authentique par rapport au sujet qui en est l'auteur, mais bien plutôt à la considérer et à la comparer en fonction de sa correspondance ou non avec ce qu'elle aurait dû être, c'est-à-dire à la norme du jugement. Le jugement de valeur sur les actions concrètes ne dit pas ce que les choses sont, mais ce qu'elles devraient être, ou mieux: ce qu'elles auraient dû être..

L'expression utilisée révèle que ce jugement est toujours a posteriori, car il n'est pas possible de juger les choses avant qu'elles ne soient. Il tombe sous l'évidence qu'il n'est pas possible de juger les actions avant qu'elle n'aient été commises. Mais ce jugement se fait toujours par rapport à une norme qui régit l'action, si le jugement est positif, ou qui aurait dû la régir, si le jugement est négatif. Ainsi seront appelées morales les actions qui sont conformes à cette norme, et im-morales celles qui lui sont contraires.

La norme apparaît donc bien comme ce terme commun que la matérialité morale exige, cette commune mesure à laquelle les actions morales et immorales sont rapportées, et qui rend compte à la fois de ce qui les lie et de ce qui les met en opposition. Alors que, dans la perspective formelle, l'amoral n'avait rien de commun avec le moral, l'im-moral et le moral sont au contraire saisissables dans une commune relation avec le contenu de valeur lié à la norme, au nom de laquelle la société et la conscience morale peuvent juger de leur contradiction.

Il existe donc une matière morale, que nous appelons la valeur, et il y a une norme morale, sous forme d'idéal, qui constitue le critère du jugement de valeur. Nous analyserons plus en détail ces notions dans la poursuite de notre raisonnement, mais nous voudrions tout d'abord montrer pourquoi il est nécessaire de les distinguer.

Cela peut surprendre, à première vue, d'utiliser la notion de valeur pour désigner la matière morale, alors que nous avons affirmé plus haut, en reprenant la notion aristotélicienne de la matière, qu'elle était en elle-même 
neutre, un simple possible pouvant être actualisé positivement ou négativement. Dire que la valeur est neutre est à première vue une contradiction, puisque la notion même de valeur implique l'absence d'indifférence et de neutralité. Cette apparente contradiction peut être éliminée en distinguant entre «la valeur par rapport au sujet», et «la valeur par rapport à la norme».

Par rapport au sujet, la valeur est la matière morale qui se retrouve dans le contenu de l'intention, elle est ce bonum apprehensum que vise l'agent dans son action. En tant que telle, la valeur ne peut être neutre, elle est toujours positive, car elle est un désirable vers lequel tend la volonté. C'est pourquoi nous devons reconnaître, de ce point de vue, que toutes les actions morales sont des actualisations de valeurs.

Mais quand ces valeurs actualisées sont considérées non plus du point de vue du sujet qui les vise, mais de la norme qui les juge, elles sont mises en relation avec un critère extérieur aux actions dans lesquelles les valeurs sont actualisées. C'est pourquoi une valeur, toujours positive aux yeux du sujet, peut être jugée négative par rapport à cette norme. C'est donc par rapport à cette norme que la valeur, en tant que matière de l'action morale, n'est pas déterminée et peut être considérée comme neutre.

Nous comprenons dès lors pourquoi, en faisant de la valeur la matière de la morale, nous découvrons la nature du conflit fondamental de la morale elle-même. C'est un conflit de valeurs entre le sujet et la norme, entre le sujet qui ne peut viser intentionnellement que des valeurs positives, et une norme qui peut juger ces valeurs comme "positives» ou «négatives» en dehors de toute référence au sujet. C'est, en d'autres mots, le conflit de l'objectivité et de la subjectivité des valeurs morales.

\section{L'objectivité morale}

Si la première dimension de la morale, la dimension formelle, se caractérise par la subjectivité essentielle de l'action, peut-on affirmer que la deuxième dimension, de nature matérielle, se caractérise par une essentielle objectivité? Une telle affirmation pourrait facilement satisfaire l'esprit, car elle donnerait à la logique de notre recherche une structure équilibrée. Mais cet équilibre ne nous semble pas suffire à justifier l'existence d'une objectivité morale. Nous devons la démontrer de façon plus précise.

La matière morale, ou valeur, reçoit, nous l'avons vu, des significations différentes, positive ou négative, non pas en fonction de ce qu'elle est en elle-même en tant que valeur, mais en fonction de sa relation avec le sujet d'une part et de la norme de l'autre. Dans sa relation avec le sujet, la valeur, ou contenu de l'intention, se présente toujours comme positive. Il y a donc bien une matière morale dont le caractère est déterminé par la subjectivité. 
Il n'est donc pas possible d'affirmer que la morale est nécessairement objective dans sa dimension matérielle.

D'autre part, quand on considère la matière morale dans l'action ellemême, indépendamment du sujet, on peut lui donner une qualification positive ou négative sans tenir compte de la subjectivité de l'agent. S'il y a objectivité, dès lors, elle ne se trouvera pas dans la matière morale seule, mais dans le critère du jugement moral par lequel cette matière est qualifiée. Or ce critère est la norme. L'objectivité morale ne vient donc pas de la matière morale, ou de la valeur, mais du jugement dont cette matière est l'objet.

\section{C'est dans la norme que se trouve l'objectivité morale}

Nous devons donc nous demander, maintenant, quelle est la nature de cette objectivité, c'est-à-dire par rapport à quoi et à qui elle est objective. Ceci exige que l'on se pose des questions sur la nature de la norme, ou sur son origine. Deux attitudes se proposent à première vue pour répondre à cette interrogation. Nous devons rapidement en analyser la pertinence.

La première position possible sur la question de l'origine de la norme, consiste à concevoir celle-ci comme découlant de la nature de l'homme. Une telle position, cependant, - nous croyons l'avoir démontré -, mettrait en cause la caractéristique formelle et essentielle de la morale, la subjectivité. Certes, il pourrait être envisageable de fonder la morale objectivement, mais celle-ci ne serait plus une morale satisfaisante pour la raison, car il n'y aurait plus de signification dans les concepts par lesquels l'esprit appréhende le phénomène moral, et la différence entre les négations «moral - a-moral» et «moral - im-moral» serait anéantie. Si l'objectivité de la norme était assimilable à l'objectivité de la nature universelle, ou de l'essence générale de l'homme, l'action a-morale, définie comme l'action irresponsable parce que déterminée par les conditions psycho-physiologiques des individus, et qui serait la plus conforme à cette norme «naturelle» puisque la plus proche de ses déterminations causales, serait la seule qui pourrait être appelée morale dans un jugement de valeur. L'affirmation de la liberté devrait être alors jugée im-morale, et les mots n'auraient plus de sens.

Certes, il peut exister des actions considérées positivement morales par le jugement de valeur selon le critère de la norme, mais qui demeurent a-

29 Bergson, Les deux sources de la morale et de la religion, p. 99. Le "behaviourisme», par exemple, considère que cette morale par dressage peut représenter toute la morale, et les structures du comportement qui en résultent constitueraient également la nature «maléable» de l'homme. 
morales en fait puisque le sujet n'y engage pas sa responsabilité. Bergson, par exemple, indiquait qu'il y avait une morale construite sur le dressage, "faite d'habitudes impersonnelles" ${ }^{29}$, dont les actions sont approuvées parce qu'elles se présentent comme «matériellement» morales. Kant, pour les qualifier, parle d'actions légales, celles qui sont conformes au devoir, mais qui ne sont pas vraiment morales à ses yeux parce qu'elles ne sont pas faites «par» devoir. Ainsi, en situant l'objectivité morale dans l'objectivité des déterminations naturelles, on limite la perspective morale au seul contenu matériel et l'on doit nécessairement conclure que l'a-moralité formelle peut être la source de la moralité matérielle. Nous croyons qu'il est impossible de soutenir une telle position.

La deuxième approche consiste à rechercher l'objectivité de la norme du jugement moral dans l'action elle-même, sans tenir compte du sujet qui en estl'auteur, et prendre cette norme non pas comme un critère de valeur, mais un principe de détermination causale, c'est-à-dire comme principe d'explication. La norme se substituerait, pour ainsi dire, à l'agent individuel. Or nous avons déjà souligné la signification réductrice de toute explication de l'action morale par ses déterminations particulières. La norme deviendrait, dans ce cas, un principe explicatif pour chaque action individuelle prise isolément, principe qui devrait être renouvelé pour le jugement de chaque action individuelle. Nous n'aurions plus alors une seule norme au nom de laquelle il serait possible de déclarer une action «morale» et une autre «immorale», puisque chaque action demanderait son propre principe normatif. Les premières actions n'auraient alors plus rien de commun avec les autres. Nous croyons, au contraire, pour qu'il y ait un jugement moral de valeur, que le critère du jugement doit être extérieur à l'action jugée. La norme doit donc être distincte d'un principe explicatif intrinsèque.

L'objectivité de la norme ne se trouve donc ni dans la nature humaine en général, car la matière morale se trouverait en contradiction avec sa forme, ni dans le contenu de l'action individuelle, car il faudrait disposer d'autant de normes qu'il y a d' actions à juger. Nous en conclurons donc qu'il ne faut pas chercher l'objectivité de la norme en dehors du fait même du jugement de valeur. Ce jugement de valeur, en effet, n'est pas un jugement du sujet sur sa propre action (dans ce cas la norme ne se distinguerait par du contenu de l'intention), mais c'est avant tout un jugement d'autrui portant sur «mon» action. C'est donc dans le rapport essentiel de la moralité avec autrui, dans sa nature sociale, que se trouve cette objectivité de la norme au nom de laquelle la morale est jugée.

Certes, les autres, la communauté de ceux qui constituent mon «autrui», et qui se proposent, ensemble ou séparément, comme témoins et juges de mon action, sont aussi des sujets, ce sont aussi des «moi» qui participent à la "subjectivité». C'est pourquoi, pris en tant qu'individus, leur jugement ne peut être considéré en lui-même comme parfaitement objectif. Nous devons donc situer ce jugement dans le cadre d'une relation spécifique entre 
eux et moi. Ce que nous appelons l'objectivité morale, (que nous distinguons de l'objectivité naturelle, celle du monde extérieur à la conscience), doit être considérée comme une objectivité par rapport à moi, auteur unique et irremplaçable de mon action, et non pas une objectivité par rapport à la subjectivité en général, ce que nous pourrions appeler la communauté des «sujets-autrui».

En d'autres termes, s'il y a une objectivité morale, si la norme a un fondement non subjectif, c'est

parce que l'homme n'est pas seul, son action est toujours une action qui concerne autrui.

La norme est objective parce que «mon» action doit toujours tenir compte des autres. Nous pourrions dire également que si l'objectivité morale est objective par rapport à «moi», elle n'est pas entièrement objective par rapport à «nous». C'est pourquoi nous appelons cette objectivité une «objectivité intersubjective».

En définitive, quand nous disons que le conflit moral est un conflit entre l'objectivité et la subjectivité des valeurs morales, nous pensons à un conflit entre «mes» valeurs qui constituent le contenu de mon intention, et "vos» valeurs qui fondent l'objectivité de la norme. Ce conflit dure aussi longtemps qu'il ne se résoud pas dans la création d'une objectivité intersubjective de «nos» valeurs, dans lesquelles la norme fondée dans le groupe et la matière de mon intention se rencontrent dans un dépassement de soi.

Mais ces valeurs qui sont «nôtres» ne sont, en fait, ni plus tout à fait «miennes», ni plus tout à fait «vôtres». C'est pourquoi l'objectivité intersubjective de la morale n'est pas une objectivité de la nature qui nie le sujet, ni celle d'une société préétablie et immuable qui m'emprisonne, mais celle d'une communauté de sujets en relation d'interaction à travers laquelle moi et les autres, le sujet et les sujets-autrui, se transforment mutuellement par le jugement qu'ils portent les uns sur les autres. L'objectivité de la norme morale est donc respectueuse du sujet en le défiant et en l'appelant à se dépasser.

En conclusion, nous dirons que l'action morale, non pas soumise à la norme, mais stimulée par elle, doit être doublement morale. Elle doit l'être formellement, c'est-à-dire qu'en elle le sujet doit pouvoir s'exprimer totalement; le «moi» de chacun doit pouvoir s'y retrouver dans ce qu'il a de plus intime et de plus libre. Mais elle doit aussi être matériellement conforme à l'objectivité que constitue la présence d'autrui dans le choix des finalités de mon action. La morale n'est donc ni exclusivement subjective ni exclu- 
sivement objective. Elle n'est ni la manifestation d'un sujet absolu et isolé, ni la soumission du sujet à un ordre établi. Elle est à la fois la création du sujet et d'autrui dans cette matière morale totale que sont «nos» valeurs. 


\section{Chapitre 4}

\section{L'obligation morale vécue (analyse existentielle)}

Un Homme est à lui seul, dans sa passion de se construire inimitable, une civilisation.

Marcel Moreau

Discours contre les entraves

En raison de l'ambiguité de la notion d'obligation qui lui vient de la double dimension de la morale, il n'est pas possible de dire «ce» qu'elle est, de la définir ou de la décrire comme un objet. En faire un objet, en effet, reviendrait à en retirer la présence essentielle et formelle du sujet, et l'obligation serait alors a-morale. Procéder, par contre, à une révélation progressive et tâtonnante de son contenu, ou de ses contenus divers à travers ses manifestations multiples dans la vie même du sujet qui se découvre, l'aborder phénoménologiquement dans l'existence concrète de l'homme, c'est quitter le domaine de l'analyse conceptuelle pour se plonger dans l'expérience directe de la vie. Nous aborderons donc l'obligation de manière vécue et existentielle.

L'existence d'une obligation vient du fait que l'action morale n'est pas une réalité donnée, mais un projet à construire. C'est parce que le sujet se découvre comme la source autonome de quelque chose qui n'est pas, mais demande à être, qu'il prend conscience de lui-même comme sujet du devoir. De la même façon, on comprend que la découverte de l'obligation peut se présenter elle-même comme une authentique obligation. Le devoir nous fait un devoir de l'inventer. 
L'obligation n'est pas une réalité cachée et préexistante qu'il s'agirait de dévoiler, comme on découvre une perle blottie dans le secret d'un coquillage, c'est au contraire un devenir qui s'élabore à travers les prises de conscience successives dont elle est la visée. Ainsi l'obligation jaillit, pour ainsi dire, de l'action morale. Elle en est le produit beaucoup plus que la source. Car, nous l'avons vu, la morale n'est pas à rechercher dans un monde objectif de choses, mais dans le dynamisme de cette «objectivité intersubjective» où chaque sujet doté de responsabilité contribue à la création des valeurs dans une conquête de soi-même, par et avec les autres.

L'obligation se construit donc peu à peu, existentiellement, à travers des "obligations particulières» qui doivent être considérées comme des expériences morales "vécues» dans lesquelles la prise de conscience d'un "devoir-être» amène le sujet à dépasser sa condition concrète et à chercher, au delà des apparences et du monde empirique, une obligation idéale dans laquelle il trouvera son propre épanouissement.

\section{L'action du «moi»: l'obligation d'être libre}

Dans Le Diable et le Bon Dieu, la pièce de Jean-Paul Sartre, nous assistons à une scène où le héros du drame, GOETZ, illustre parfaitement l'une des exigences de l'obligation. C'est celle qui consiste à affirmer l'action comme «mienne», et à révéler ma conscience morale à autrui en lui donnant un contenu de valeur.

Au moment où GOETZ décide de prendre la ville de Worms qu'il assiège, CATHERINE lui demande le pourquoi d'une telle décision:

GOETZ: Parce que c'est mal.

CATHERINE: Et pourquoi faire le Mal?

GOETZ: Parce que le Bien est déjà fait.

CATHERINE: Qui l'a fait?

GOETZ: Dieu le Père. Moi, j’invente.

La motivation de GOETZ semble claire. Son but est de faire une action qui lui soit totalement personnelle, que personne d'autre ne pourrait revendiquer à sa place. Faire le Mal lui paraît une occasion unique. Choisir le mal, c'est affirmer son originalité individuelle, c'est inventer, c'est manifester son «moi». Etre libre, c'est ne pas être comme les autres, ne pas faire ce qui est déjà fait, choisir un autre contenu de valeur et voir dans le renversement des valeurs la source de la liberté. Pourtant, quelques instants plus tard, GOETZ change sa décision quand il apprend que le mal est aussi fait par 
d'autres et qu'il ne lui apparaît plus comme spécifique de son propre moi. Donc il va changer:

«Tu m'apprends que le Bien est impossible, je parie donc que je ferai le Bien: c'est encore la meilleure manière d'être seul. J'étais criminel, je me change: je retourne ma veste et je parie d'être un saint».'

L'intention de GOETZ est claire. Ce qu'il vise par dessus tout, c'est d'être «seul», c'est-à-dire de ne devoir à personne et à rien la responsabilité de son action. Même s'il participe à l'universel par sa condition humaine, le sujet doit marquer son être par une rupture morale avec le lien social s'il veut être lui-même. "Il ne peut y avoir de réflexion autonome, de création artistique ou intellectuelle, de révolte morale, que si une faille originelle empêche le sujet individuel de coïncider avec «l'identité» que lui confère son appartenance sociale ${ }^{2}$.

La liberté, finalement, n'est pas liée à vouloir le Bien ou à vouloir le Mal, mais à ne vouloir l'un ou l'autre que par soi-même, en rupture avec l'attente ou la pression sociale. Mais peut-on être libre en manifestant une complète indifférence au Bien et au Mal? Certes, il est impossible d'agir sans faire le Bien ou le Mal, sans actualiser une valeur, ce que GOETZ reconnaît, mais il sait aussi que l'authenticité de l'action ne dépend pas de ce contenu de valeur. Pour tenter de le prouver, il décide de jouer aux dés. S'il gagne, il fera le Mal; s'il perd, il fera le Bien. Il joue et il perd. Mais la scène se termine sur une dernière intervention de CATHERINE qui révèle en riant:

- «Il a triché! Je l'ai vu, je l'ai vu, il a triché pour perdre!»

Cet exemple illustre deux conditions fondamentales de la révélation de la liberté morale. D'abord que la liberté ne se trouve pas dans le contenu de valeur de l'acte: ni dans le Mal, ni dans le Bien. On comprend dès lors que les morales du «renversement des valeurs» ne peuvent être réellement créatrices de liberté. Il ne suffit pas d'être "contre" pour avoir raison. Car toujours l'homme remplace les valeurs dominantes qu'il rejette par d'autres valeurs qui, à plus ou moins brève échéance, rempliront le même rôle de domination. «Je suis là, assis», dit Zarathoustra, «et j'attends, entouré de

1 Jean-Paul Sartre, Le Diable et le bon Dieu, (1951), Acte I, Troisième tableau, Scène IV.- VI

2 André Gorz, Métamorphose du travail, Quête du sens, p. 215 
vieilles tables brisées, et aussi de nouvelles tables à demi-couvertes d'inscriptions». On n'affirme pas sa liberté en rejetant un contenu de valeur particulier, car cela se résumerait à choisir une autre valeur qui, à son tour, pourrait devenir aussi menaçante pour la liberté. La liberté morale ne se trouve pas dans la matière morale, mais dans sa forme. Les valeurs n'assurent pas la liberté. A la suite de l'exemple de GOETZ, la liberté apparaît comme indifférente aux valeurs.

Il n'empêche que, formellement, il est impossible d'agir en toute liberté sans choisir une finalité pour son action, sans viser un but, sans actualiser une valeur. La première obligation morale est donc celle de choisir, et de le faire pour être libre. Ne pas choisir est a-moral, c'est donc renoncer à soimême. C'est pourquoi le héros de Sartre ne peut accepter la décision du jeu: il doit tricher. Admettre que les dés, le hasard ou Dieu par le truchement des dés, décident de choisir le Bien ou le Mal à sa place, serait, aux yeux de GOETZ, abandonner sa propre liberté. Il ne peut donc être à la fois libre et indifférent. Il doit décider lui-même de faire le Bien ou le Mal; c'est pourquoi il triche.

Certes, il aurait pu choisir de faire le Mal, et en ce sens nous reconnaissons que la liberté est indifférente aux valeurs, ou du moins au contenu d'une action particulière. Mais il n'y a pas d'indifférence de la liberté devant la nécessité d'affirmer l'existence des valeurs.

La liberté, c'est l'obligation de choisir.

On pourra dire que GOETZ aurait pu ne pas choisir, et laisser les dés décider pour lui. Mais dans ce cas il aurait quand même choisi de ne pas choisir. Il n'y a pas moyen d'échapper à l'obligation du choix. La véritable indifférence aux valeurs consisterait à croire qu'elles existent en dehors de nous, qu'elles sont des réalités sur lesquelles notre libre choix n'a aucune influence, des "êtres-en-soi» dont l'actualisation ne dépend pas de notre libre engagement. Les valeurs elles-mêmes deviendraient alors indifférentes à notre liberté et à notre intention, car leur être, en tant que valeurs, ne dépendrait pas de l'action humaine pour s'actualiser. C'est en ce sens qu'il faut comprendre la première révolte de GOETZ contre le Bien «qui est déjà fait». Mais au moment où il découvre que les valeurs ne sont pas nécessaires, déterminées par un ordre des choses préexistant à son propre choix, il peut choisir le Bien qu'il avait rejeté. En choisissant le Bien, il choisit d'être libre. Puisque ce choix est obligatoire, sinon son action n'aurait pas été sienne, nous dirons donc qu'il y une obligation d'être libre, une obligation

3 Nietzsche, Zarathoustra, III, «Des vieilles et des nouvelles tables» 
de se choisir libre. Et cette obligation est authentiquement morale puisque l'action qui n'est pas libre n'est pas morale.

Cette liberté «obligatoire», dirons-nous, demeure cependant formelle. Sartre lui-même le reconnaît dans un autre texte écrit antérieurement à l'oeuvre dramatique citée ici. Reconnaissant qu'en dépit de la variabilité du contenu de la morale il y a une certaine forme de cette morale qui est universelle, il conclut de la façon suivante: «Le contenu est toujours concret, et par conséquent imprévisible; il y a toujours invention. La seule chose qui compte, c'est de savoir si l'invention qui se fait se fait au nom de la liberté»". La liberté est-elle affirmée ici comme la forme universelle de la morale? Tandis que, pour Kant, l'acte moral est celui qui est fait "par devoir», il est pour Sartre celui qui est accompli «au nom de la liberté». Le devoir kantien et la liberté sartrienne jouent ainsi le même rôle formel dans la construction de la morale.

Il y a donc une première manifestation de l'obligation qui est l'obligation d'être libre.

\section{Les temps de l'action : l'obligation de créer la moralité}

L'action morale est une action dont on peut dire qu'elle est bonne ou mauvaise. De ce fait, tout agir humain est producteur de valeur pour autrui. Il participe à la construction de l'objectivité intersubjective, une réalité morale qui n'est plus exclusivement celle du sujet. La liberté morale, même si elle ne peut être exercée que dans ma seule conscience, n’implique pas nécessairement la solitude de l'être.

Pour le comprendre, nous allons distinguer trois moments de l'action morale. Cependant nous les considérerons dans l'ordre inverse de leur déroulement chronologique, à savoir, successivement:

- «l'action comme achevée», - constitutive de ce que nous appelons la réalité morale objective, ou la moralité -,

- I'action «en train de se faire», - qui représente l'agir propre du sujet moral -, et

- «l'action comme projet», - qui s'inscrit préalablement à l'agir dans l'intention du sujet.

L'action achevée, accomplie, est celle dont nous avons dit qu'elle faisait l'objet d'un jugement de valeur. Elle n'intéresse le jugement que par sa moralité ou son im-moralité, en tant que valeur objective. Elle est à la

4 Jean-Paul Sartre, L'existentialisme est un humanisme, p. 86 
disposition, nous pourrions même dire qu'elle «appartient», à la communauté des sujets-autrui qui la jugent. Elle est ce «Bien ou ce Mal» qui est déjà fait, comme dit GOETZ. C'est une réalité saisissable, connaissable, comme l'oeuvre d'un artiste.

Certes il n'est pas possible d'ignorer l'artiste créateur dans la considération de son oeuvre; on retrouve objectivement sa présence dans l'objet qu'il a créé. Mais cette oeuvre, une fois achevée, lui échappe, elle est devenue publique, objective, on peut s'en saisir comme d'un objet. C'est ainsi que l'action morale achevée devient une réalité objective, indépendante de son auteur, constitutive de l'environnement moral dans lequel nous vivons. C'est ainsi qu'il est possible d'affirmer que le Bien ou le Mal «existe» dans la communauté des hommes.

L'action «en train de se faire», elle, ne peut être séparée du sujet qui agit. C'est l'agir même du sujet. C'est le moment où le sujet est vraiment conscient d'être le maître de son action. C'est l'artiste en train de créer. C'est le moment où l'homme vit pleinement ce qu'il fait. C'est une expérience très intense, aussi le sujet s'y attache souvent davantage qu'à l'action accomplie. C'est à cette action que s'attachent les théories morales de «l'agir pour l'agir», et c'est à ce stade que se situerait, s'il était possible, l'acte gratuit, celui qui n'a pas d'autre finalité que l'acte en lui-même. Le sujet y trouve la plus pure expression de lui-même. Il s'y découvre vraiment comme étant ce qu'il fait, comme étant réellement bon ou mauvais. La valeur de l'action n'est pas saisie comme la valeur objective d'un fait, mais comme la valeur d'être de l'agent. Le sujet n'a pas l'impression de faire le Bien ou le Mal, mais d'être bon ou mauvais.

Toutefois, cette distinction n'est possible qu'au niveau d'un discours logique et d'une analyse abstraite. Les deux moments sont inséparables dans la réalité. L'action «en train de se faire» n'est que le devenir de l'action accomplie, elle est, de ce fait, déjà une réalité du monde moral d'autrui. C'est pourquoi le sujet est toujours un être agissant pour les autres, il ne peut s'enfermer seul dans son action comme dans un pur agir. Qu'elle soit accomplie ou en train de se faire, l'action est donc soumise à la norme morale, le critère objectif par lequel elle sera jugée. L'homme ne peut échapper à l'obligation de créer une réalité morale soumise à la norme.

Cette obligation concerne donc autant l'action, «chose terminée» que l'action «en train de se faire». Il est possible de dire que le sujet prend conscience d'être bon ou mauvais au moment du devenir de l'action, parce qu'il anticipe, pour ainsi dire, le résultat final de son action qui sera le bien ou le mal selon la norme. En fin de compte, le sujet découvre ce qu'il est par ce qu'il fait. La norme devient, ainsi, le critère de la découverte de la valeur du sujet lui-même. Je suis donc obligé de créer la réalité morale pour autrui afin de devenir moi-même valorisé à mes propres yeux. La valeur morale de mon être n'existe pas en dehors de la valeur morale de mon action. L'obligation de créer un monde de valeurs devient la condition de mon propre devenir. 
L'action comme "projet» est une action que l'on considère par anticipation comme achevée et sur laquelle on porte à l'avance un jugement selon la norme. Certes, ce projet ne suit pas forcément les indications d'une norme posée a priori dans l'absolu. La norme n'est pas donnée dans l'objectivité pure, mais construite par cette réciprocité des consciences qui constitue l'objectivité intersubjective de la moralité. Créateur de valeurs, l'homme crée aussi la valeur idéale qu'est la norme. L'action comme projet est donc aussi création; c'est un jugement de valeur anticipé par la visée d'une norme.

La philosophie classique proposait la même chose en disant que la volonté ne pouvait vouloir que le bien. Kant a exprimé la même obligation en demandant à l' homme d'agir de telle façon que le principe de son action puisse être érigé en loi morale universelle. Sartre, pour sa part, souligne la même obligation quand il écrit: «Il n'est pas un de nos actes qui, en créant l'homme que nous voulons être, ne crée en même temps une image de l'homme tel que nous estimons qu'il doit être. ... ce que nous choisissons, c'est toujours le bien, et rien ne peut être bon pour nous sans l'être pour tous» ${ }^{5}$. A sa façon GOETZ disait la même chose en affirmant: «Le Mal, ça doit faire mal à tout le monde».

L'obligation de créer est donc l'obligation première de créer une norme par son projet, norme par laquelle notre action accomplie pourra être jugée, et dans laquelle nous découvrons l'homme que nous voulons être. Il n'y a donc pas seulement une obligation fondée dans le contenu de la norme, comme quelque chose qui s'impose à nous, il y a premièrement une obligation plus fondamentale qui demande à l'homme de créer l'obligation qui va révéler le contenu de la norme. L'obligation n'est pas la conséquence de l'existence d'une norme, elle en est la condition. Nous ne sommes pas seulement obligés de suivre une morale, nous avons aussi l'obligation de créer la morale.

\section{Les causes de l'action : l'obligation de se faire soi-même}

"Le chemin de l'enfer est pavé de bonnes intentions», dit le proverbe populaire. En effet il y a, dans la vie morale, une foule d'actions projetées qui s'épuisent et meurent au niveau de l'intention sans voir le jour. Or, si le sujet renonce à l'action, le Bien comme le Mal n'existeront pas. Sans l'action la moralité n'existe pas dans l'objectivité intersubjective. C'est l'agir du sujet, son engagement existentiel, puisqu'il implique un pouvoir d'agir sur l'existence elle-même, qui est source de la réalité morale. Il ne

5 lbid., p. 25 
suffit pas d'imaginer et de contempler un ciel idéal de normes abstraites préétablies pour assurer l'existence concrète de la moralité. Il faut une action dont le sujet est le premier acteur. Or le sujet se construit lui-même en agissant. Dans l'action accomplie, dans la moralité réalisée, objective, il y aura toujours le sujet lui-même, transformé.

Pour comprendre cette transformation du sujet à travers la création de la moralité réelle, nous devons rattacher les notions que nous avons dégagées jusqu'ici à une doctrine du devenir de l'être. Ceci nous semble nécessaire pour rendre compte du dynamisme créateur des trois moments de l'action.

Nous retiendrons, pour expliquer le devenir du sujet, des éléments tirés de l'hylémorphisme aristotélicien, sans toutefois suggérer que la cosmologie que ces principes soutiennent est nécessaire, comme fondement métaphysique de notre approche. L'objectivité morale dont nous parlons est «intersubjective», non assimilable à l'objectivité pure des objets. Il ne s'agit pas d'une "physique», mais d'une philosophie pratique. L'être en devenir analysé ici n'est donc pas un être donné dont le développement échappe à notre volonté, mais un être moral construit par la volonté libre de sujets.

Selon l'hylémorphisme aristotélicien, tout être est composé de deux éléments complémentaires, une forme et une matière. C'est l'ensemble de ces deux éléments qui constitue l'essence complète de l'être en question. La matière seule, prise en tant que telle, n'est pas l'être; elle n'est qu'une simple puissance à être, la possibilité indéterminée de devenir quelque chose. En d'autres mots, elle est comme un appel. Toutefois, cette matière n’est «rien» de réel tant qu'elle n'est pas «informée», tant que la forme son essence en termes aristotéliciens - ne lui donne pas une consistance reconnaissable. L'existence ne commence donc qu'avec «l'information d'une matière", qui est le passage de la puissance (matière indéterminée) à l'acte (l'être totalement informé). L'être n'atteint donc sa perfection qu'au moment de la réunion, ou fusion existentielle, en une seule existence individualisée de la matière et de la forme. La totalité des conditions de l'être ne se comprend dès lors que dans son devenir, dans ce passage et cette transformation qui va de la puissance à l'être actualisé.

En appliquant cette perspective au devenir moral, dans la ligne des distinctions que nous avons établies au chapitre précédent, nous dirons que la "matière morale», la valeur, que nous avons appelée "norme» sous sa forme idéale et objective, n'existe pas, elle est «néant», tant qu'elle n'est pas informée de la subjectivité de l'agent. Il n'y a pas de normes existantes avant qu'elles ne soient reconnaissables dans un acte moral accompli. Or cet acte n'existe pas sans l'intervention du sujet. En d'autres termes, l'actualisation morale exige la présence des deux dimensions de la morale.

Il ne faut cependant pas interpréter ce devenir en faisant une simple transposition de l'hylémorphisme aristotélicien. Pour Aristote, en effet, il n'y a pas d'être donné, réel, en dehors de l'information de la matière. Ni la 
forme ni la matière n'existent en soi, indépendamment l'une de l'autre. Elles n'ont pas d'existence propre et il n'y a pas plus de force d'actualisation dans la matière seule qu'il n'y a d'existence réelle dans la forme isolée. Matière et forme sont pure disponibilité à une existence actualisée par leur fusion.

Dans la réalité morale, forme et matière tendent à une existence indépendante l'une de l'autre. D'une part la matière se propose comme réalité autonome, puisque c'est par la norme seule que l'on distingue le moral de l'im-moral, et d'autre part le sujet, forme indispensable, qui prend conscience de lui-même comme irréductible à toute objectivation quelconque, s'affirme contre la norme. Forme et matière, en morale, ne semblent donc pas s'appeler mutuellement à l'existence, mais au contraire à se dresser l'une contre l'autre et à se repousser en affirmant ouvertement ce qui les distingue.

C'est en analysant les caractéristiques de ce que nous avons appelé les moments de l'action morale que nous voyons comment il est possible de permettre au devenir moral d'échapper à l'impasse dans laquelle notre analyse conceptuelle a pu donner l'impression de l'enfermer. «L'action comme projet» ne peut devenir une «action accomplie», donc participer à la réalité morale, que par l'agir du sujet, donc par «l'action en train de se faire», qui est la manifestation pleine et authentique du pouvoir actualisant du sujet. De ce fait, nous comprenons que le sujet n'est pas seulement la forme de l'action, mais qu'il en est avant tout le "faiseur», l'agent actualisant. L'action est morale non seulement parce qu'elle est mienne dans sa forme, mais parce que $j$ 'en suis concrètement l'artisan.

Le sujet est donc à la fois forme et cause de l'action.

La théorie des quatres causes de la philosophie classique est utile à ce stade de l'explication. Chez Aristote, pour qu'il y ait passage de la puissance à l'acte, il faut l'intervention d'une cause extérieure qui ne soit ni la puissance ni l'acte, ni la matière simple ou la forme pure. La statue ne peut sortir de la pierre que si le sculpteur intervient, que s'il impose à la matière amorphe de la pierre une forme par laquelle elle devient statue. Le sculpteur est la cause efficiente de la statue. Sans l'intervention de cette cause, la matière serait restée sans forme, une pierre sans signification, et l'image, la forme, serait demeurée inexistante. Toutefois, sans la potentialité de la matière et de la forme, la cause efficiente n'aurait pas pu agir non plus.

Dans le devenir moral, le sujet est lui-même la cause efficiente, celui qui commet réellement l'action, celui qui en est l'auteur. Mais il ne peut le faire qu'en fondant son intention sur la cause matérielle et la cause formelle qui lui sont offertes. La cause matérielle est la valeur idéale proposée dans la norme; la cause formelle, c'est lui-même en tant que sujet. Pour l'être humain, en conséquence, s'engager dans une action n'est pas seulement 
actualiser une forme et une matière extérieures et préexistantes à son être propre, comme la pierre et l'image ne sont pas le sculpteur, mais c'est se réaliser lui-même par la matière qui est proposée.

Quant à la quatrième cause, la cause finale, elle se retrouve dans la réalité morale sociale dans laquelle le moi et les autres se rencontrent en tant que membres actifs de cette objectivité intersubjective qu'est la société morale.

On comprend donc que les sujets - tous les hommes - non seulement interviennent dans l'action morale à l'instant de «l'action en train de se faire», comme cause efficiente qui fait passer la moralité de la puissance à l'acte, mais se retrouvent dans l'essence même de la moralité, comme forme de «l'action morale accomplie». C'est pourquoi, en raison de ce double rôle "causal" de l'homme dans le devenir de la moralité, cause efficiente et cause formelle, nous disons que le sujet a l'obligation de se faire lui-même en accomplissant l'action morale. L'homme n'est pas seulement la source du devenir moral en tant qu'agent, il en est également le produit, en tant que forme de la moralité.

Le sujet se crée ainsi lui-même en actualisant la puissance à être qu'est la valeur comme matière morale. "L'action bonne, par définition, rend l'agent qui la produit bon. L'action bonne, qui est bonne en elle-même (nous dirons selon la norme), parfait ou achève mon propre être ${ }^{6}$. Le devenir de l'homme n'est donc pas indifférent aux valeurs.

\section{Le conflit moral : l'obligation de se dépasser}

Si l'homme prend conscience d'être bon ou mauvais dans «l'action en train de se faire», il comprend aussi qu'en tant que forme de l'action accomplie, il devient, dans la moralité actualisée, le porteur authentique du Bien et du Mal. Dans la pièce de Sartre déjà citée, GOETZ déclare: «Le Mal, ça doit faire mal à tout le monde. Et d'abord à celui qui le faits ${ }^{7}$.

L'homme ne peut, donc, à aucun moment, demeurer indifférent aux valeurs. Non seulement il a l'obligation de choisir, au moment de la visée ou de l'intention, sinon son action ne serait pas libre, mais il ne peut non plus ignorer les valeurs dans l'ordre de la moralité actualisée, puisqu'il devient lui-même un élément constituant de la réalité qu'il appelle à l'existence. Cette réalité n'est donc pas «pur objet», puisqu'elle le constitue lui-même en même temps dans son être. L'homme invente, certes, puisqu'il peut, dans chaque situation concrète, choisir entre le bien et le mal. Mais

6 Jacques Maritain, «Neuf leçons sur les notions premières de la philosophie morale», Oeuvres complètes, Vol IX, p. 780

7 Le Diable et le bon Dieu, Ibid., scène IV. C'est nous qui soulignons. 
ce pouvoir d'invention n'est pas un pouvoir de création ex nihilo à l'instar du pouvoir divin. Certes, le sujet prend conscience, dans l'action morale, d'un pouvoir extraordinaire puisqu'il peut décider de l'existence du Bien et du Mal par sa seule volonté et son libre choix. Mais ce n'est pas un pouvoir total de création, car ce «créateur moral» qu'est le sujet ne peut entièrement se libérer de l'objet créé, ou lui devenir indifférent, puisqu'il se retrouve luimême dans ce qu'il fait. Existentiellement, l'homme n'est ainsi jamais "par delà le Bien et le Mal».

Il l'est peut-être seulement dans ce bref et fugace instant de l'action comme projet, au moment où il a l'impression de dominer les valeurs avant de les choisir. Il faudrait mieux analyser l'ivresse de l'incertitude qui peut saisir le sujet à cet instant privilégié où l'homme, prenant conscience que tout est peut-être encore possible par rapport aux valeurs, peut avoir le sentiment d'être Dieu! Mais dès qu'il agit, il se constitue lui-même dans ce qu'il fait et se trouve totalement plongé dans le Bien et le Mal. La liberté humaine n'est donc ni absolue ni divine, car elle ne dispose pas du pouvoir de créer un monde extérieur qui subsiste en dehors de son acte créateur. L'homme se fait lui-même dans ce qu'il crée, il ne s'en détache jamais, car il se retrouve dans l'objet qui résulte de son libre choix.. Il n'est aucunement un grand horloger qui pourrait prendre des distances avec le mécanisme qu'il a mis en branle et se reposer dans la contemplation d'une réalité totalement extérieure dans laquelle il ne se sentirait pas impliqué. Il n'y a pas de secteurs de la vie humaine, économiques ou politiques, par rapport auxquels l'homme pourrait faire des choix dans lesquels il ne serait pas mêlé directement et existentiellement. L'homme ne peut pas, à aucun moment et dans aucune situation, se réfugier derrière la pureté de ses intentions, jouer les Ponce Pilate et se laver les mains des conséquences de ses actes. Il n'échappe pas à sa responsabilité.

Au contraire, dès qu'il agit, l'homme perd la souveraineté abstraite de sa propre existence, et devient pétri de Bon et de Mauvais, jugé au nom d'une norme dont il n'est pas le seul maître. Puisque son action l'amène à se créer lui-même dans la moralité qu'il actualise, puisque cette moralité est jugée bonne ou mauvaise en fonction d'une norme dont il ne dispose pas seul, puisque cette norme est la valeur idéalisée par la communauté des sujets, nous devons donc reconnaître que l'homme moral est un créateur libre qui ne peut exister moralement que par les autres. Même en se voulant inimitable, il crée une civilisation. Son acte n'est jamais une action solitaire; il est toujours cause d'un Bien ou d'un Mal qui s'impose à la société.

C'est pourquoi la condition morale est la source d'un conflit. C'est le conflit d'un être divisé entre le pouvoir de sa liberté et la nécessité de dépendre d'autrui pour vivre dans le Bien ou le Mal. C'est le conflit d'un sujet qui ne peut s'achever lui-même dans le secret de sa liberté, mais qui ne peut se parfaire qu'en se dévoilant: conflit entre le moi «pour moi», et le moi «pour autrui». 
L'idée même de conflit, aussi dramatique soit-elle, suggère la possibilité d'une solution, d'une transformation des parties impliquées en vue d'une évolution vers une situation nouvelle. Contrairement aux héros de la tragédie grecque, qui faisaient face à la pérénité d'une situation insoluble et ne pouvaient agir pour changer leur destin, les héros d'un conflit savent qu'ils se trouvent devant des choix, des actes possibles, des fins à inventer. Un conflit est par nature une situation dynamique qui tend vers un dénouement, une réalité porteuse de promesses. Disons, à la limite, qu'un conflit contient l'espoir d'un devenir. Un homme en conflit a un horizon ouvert devant lui; il y trouve la possiblité d'une création.

C'est la nature même du conflit moral. Pour en comprendre la nature, reprenons notre interprétation du phénomène moral par l'idée du passage de la puissance à l'acte. La moralité concrète n'existe pas par elle-même; elle n'accède à l'être que par l'action du sujet. Mais il n'en demeure pas moins vrai que le sujet ne pourrait l'attirer dans l'être, pour ainsi dire, que si elle ne manifestait pas, matière non encore informée, - c'est-à-dire pure valeur-norme -, un appel à l'actualisation, un penchant pour l'être, une demande à l'existence. Pour que le sculpteur, par exemple, se mette à faire la statue, il faut qu'il sente, du côté de la matière, une disposition à devenir quelque chose. Il doit y avoir un appel. Quand l'artiste le sent, il voit ce qu'il va faire; il touche la pierre de ses mains, en sent la résistance et la densité et, en raison de cette disposition de la matière à être travaillée, il entre pour ainsi dire en conflit avec elle; il est alors prêt pour commencer son oeuvre.

Il en est de même dans le domaine moral. La norme s'offre à l'agent pour la moralité, elle témoigne d'une disposition pour l'existence réelle, elle appelle l'action morale accomplie. Cet appel s'adresse à l'agent, cause efficiente possible, mais il s'adresse également au sujet en tant que cause formelle indispensable. Le conflit moral vient justement de la dualité causale de l'agent. Comme cause efficiente, l'agent est libre, maître de son action. Comme cause formelle, il est passible de devenir le résultat actualisé de l'action. Il doit donc, pour agir, s'abandonner au nouvel être qu'il va devenir. Ce que les exigences de la valeur morale impliquent, c'est une renonciation de l'homme à la subjectivité pure pour devenir une nouvelle existence revalorisée par le jugement d'autrui. Or cette subjectivité lui était apparue comme la forme essentielle de l'action morale, sans laquelle la morale elle-même n'aurait plus de sens. Il n'est pas possible de dire, dès lors, que la solution du conflit se trouve dans un renoncement à la subjectivité, car celle-ci demeure indispensable comme forme, mais dans un véritable dépassement de la subjectivité. Cela veut dire que la subjectivité résoud le conflit moral en se retrouvant dans un ordre nouveau où elle s'ouvre à d'autres subjectivités sans cesser d'être elle-même. Il ne s'agit pas d'une négation de la subjectivité, puisque le sujet lui-même demeure l'agent de ce dépassement, mais d'une véritable transformation choisie et voulue.

C'est ainsi que le conflit moral qui oppose la norme au sujet, et par là l'homme à lui-même, lui fait une obligation de se dépasser, c'est-à-dire de 
se transformer lui-même, par lui-même, dans un ordre d'existence nouveau, à la poursuite d'une dimension nouvelle de son existence et de sa vie. C'est par là que la transformation morale des sociétés est rendue possible, que le progrès moral a des chances d'apparaître dans l'histoire des hommes.

Il faut tenter maintenant de voir où se trouve la source réelle de cet appel au dépassement.

\section{L'appel d'autrui : l'obligation réelle}

L'obligation de se dépasser est la réponse à un appel en faveur d'une existence nouvelle, un devenir, un devoir être. Mais cet appel n'est pas une vague demande anonyme, une voix plus ou moins secrète, une poussée inconsciente, comme la voix de Dieu entendue par Fulliquet, ou la force de la vie dans la vision bergsonienne. Il s'agit d'un appel précis, concret, qui s'adresse au sujet moral en situation de conflit et qui s'adresse à lui seul. A partir de l'analyse existentielle que nous avons faite de l'obligation vécue, l'obligation morale nous apparaît comme l'appel que la moralité, en puissance d'être dans la norme, adresse au sujet pour lui demander de se réaliser en l'actualisant.

Mais cette description "provisoire» de l'obligation demeure très insatisfaisante. Plusieurs questions se présentent à l'esprit. Qu'est-ce que cette norme représente en fait? En quoi peut-elle me demander de me dépasser? Quelle est la nature de cette objectivité intersubjective dans laquelle nous l'avons enfermée? Pourquoi faut-il identifier cet appel à l'obligation? Répondre à ces questions, c'est se demander d'où vient en fait cette norme, décrite jusqu'à maintenant comme une valeur idéalisée par la communauté d'autrui, une valeur qui n'avait pas d'existence propre puisqu'elle avait besoin de l'intervention de l'acte du sujet pour être. Il semble difficile de comprendre en fait pourquoi ce sujet, bien réel et faisant l'expérience de sa propre existence, peut être affecté par un appel venant de ce qui n'est pas encore.

On peut répondre à ces interrogations en abordant le problème de l'existence humaine à partir de la conscience du devenir et du rôle des valeurs dans cette prise de conscience. Dire de la valeur qu'elle n'est qu'une simple matière morale qui n'accède à l'être que par l'action du sujet, c'est impliquer que les valeurs n'existent pas en elles-mêmes, qu'il n'y a pas d'être «donné» des valeurs. En d'autres mots, il n'est pas possible de concevoir des substances axiologiques préexistantes à l'action qui les fait vivre. La valeur n'est donc que l'affirmation, par l'homme lui-même, d'un devenir possible de l'homme.

Si le Bien est une valeur, c'est qu'il représente un devenir possible de l'homme. Si le Mal est une valeur négative, c'est qu'il est la négation d'un devenir possible. 
Dans cette perspective, la norme morale, cette valeur idéalisée par la communauté des sujets-autrui, n'est que l'expression du devenir idéal, tel qu'il est pensé et exprimé par une communauté donnée à une époque particulière. Il n'y a pas de norme morale absolue, car elle devrait, pour être absolue, se proposer comme existant par elle-même, ce qui l'empêcherait, par ce fait même, de se présenter comme valeur. Cette perspective n'est possible que dans la perfection divine proposée par la moralité chrétienne, qui demande au croyant d'être "parfait comme son Père céleste est parfait».

D'autre part, quand nous disons que la valeur n'est qu'une simple puissance d'être, nous n'impliquons pas non plus qu'elle puisse devenir un être réel par elle-même, comme simple résultat d'une action, une réalité subsistant par soi. La valeur a besoin non seulement d'une cause efficiente pour exister, le sujet moral, mais d'une cause formelle pour survivre, l'homme en tant que sujet.

Actualiser des valeurs ce n'est donc pas autre chose que créer l'homme selon ce qu'il pense lui-même de son devoir être, c'est-à-dire de son devenir. Mais l'homme, en tant que sujet, vit dans un monde des fins, comme dit Kant, jamais dans un monde des moyens. Il n'est donc pas possible de le décrire comme une chose, puisque rien de ce qui le constitue comme sujet ne peut être objet. Voilà pourquoi il n'est pas possible non plus de parler de son devenir selon les lois objectives de l'évolution de la nature ou des espèces, et encore moins selon les exigences d'une croissance purement quantitative.

C'est pourquoi nous avons parlé de dépassement, c'est-à-dire d'une ouverture du sujet à une réalité nouvelle dans laquelle le sujet, sans se renier en tant que sujet, vit au-delà de lui-même, en contact avec d'autres réalités qui ne peuvent ni le nier ni le rejeter car elles sont de même nature. Ainsi se dépasser, pour un sujet, c'est se retrouver dans une communauté de sujets.

L'appel de la norme, dès lors, n'est pas autre chose que l'appel d'autrui qui me demande de vivre avec lui afin de devenir moi-même plus intensément, tout en lui permettant d'être lui-même plus authentiquement.

La norme morale, finalement, est cette valeur idéale qui affirme un devenir spécifique des hommes, celui dans lequel, chacun se dépassant soimême à la rencontre de l'autre, la rencontre de cette humanité intersubjective devient une véritable création de soi et des autres dans une communauté transformée. L'obligation morale n'est donc pas l'appel d'une norme existante, c'est l'appel d'autrui, sujets incarnés comme moi dans un devenir, qui me demandent de participer à ce dépassement réciproque des subjectivités qu'est une authentique rencontre humaine.

Et cette obligation est universelle car elle demande de reconnaître, en chaque rencontre prise individuellement, la totalité du dépassement humain. En d'autres mots, il existe une obligation morale parce que l'homme 
n'est pas seul et qu'il a essentiellement besoin de l'autre pour être totalement lui-même.

C'est en autrui, finalement, que se trouve la source de l'obligation.

Et c'est pour cela qu'il n'est pas possible de la définir de façon définitive, car autrui est sujet, lui aussi, dont l'être et le mystère dépassent infiniment les concepts que la raison pourrait produire pour le décrire. Il existe, il m'appelle à moi-même, et c'est par lui seul, pour finir, que je suis obligé.

\section{La personne comme devoir-être}

Affirmer que l'obligation ne trouve sa pleine signification que dans cet appel mutuel des sujets, c'est situer le problème de l'être du sujet dans la finalité du devenir moral. Dans les différentes manifestations de l'obligation que nous avons analysées jusqu'ici, nous avons vu que l'obligation se présentait comme un appel venant d'autrui demandant au sujet de refuser la réalité donnée, de mettre en cause la réalité du monde, de la société, et même de sa propre existence, c'est-à-dire celle du sujet empirique, afin de trouver dans un au-delà du monde donné, du social et de la subjectivité, l'authentique réalité morale qui est celle d'un dépassement créateur.

Nous appelons la personne cet être du sujet transformé par l'obligation, c'est-à-dire transformé en permanence par l'appel d'autrui. Cet être de la personne est lui-même en perpétuel devenir puisque, ne se comprenant que par l'obligation, cette dernière demeure la condition essentielle de son existence. La personne est un sujet en état de perpétuel devenir par l'obligation. Si la personne, en effet, était donnée préalablement au devenir dans lequel elle se révèle, l'obligation ne se présenterait pas comme un appel, mais comme une explicitation des structures de l'existence, et la liberté ne serait qu'une négation de l'être. Il n'y a donc pas de différence entre «être» une personne et «devenir» une personne.

Ce devenir apparaît, la chose est évidente, de nature tout à fait différente de la croissance purement quantitative qui fonde l'éthique de la prospérité. La croissance quantitative peut aussi être considérée comme un devenir, mais elle ne peut en aucun cas tenir compte de la subjectivité essentielle au devenir de la personne. C'est pourquoi elle ne peut être réellement morale.

L'anthropologie morale que nous tentons de construire ne peut être limitée à l'ordre des concepts puisque cet ordre demanderait à la raison de définir la personne, définition qui serait directement remise en cause, car la personne définie devient aussitôt celle qui ne doit plus être, celle qui aurait cessé de se construire et de se dépasser en réponse à l'appel de 
l'obligation. L'anthropologie morale ne doit donc pas s'attacher à définir son objet, mais à décrire l'existence dans laquelle l'homme réalise son propre devenir. Elle doit décrire l'homme comme personne, c'est-à-dire comme action et liberté. «En «objectalisant de quelque façon que ce soit un homme, nous le perdons nécessairement de vue en tant que support de valeurs morales". ${ }^{8}$

Certes, parler de décrire la personne est déjà ambigu, comme si la personne pouvait être tirée hors de l'intimité dans laquelle elle poursuit son propre devenir. Seuls les contours saisissables de la situation existentielle dans laquelle ce devenir personnel prend place peuvent faire l'objet de cette description. Nous soulignons, «les contours saisissables», car nous reconnaissons qu'il y a une région du devenir humain qui échappe à toute description conceptuelle. En effet, puisque le devenir de la personne est nécessairement lié à la présence de l'obligation, c'est-à-dire à l'appel d'autrui, et puisque autrui lui-même est une personne en devenir, il demeure toujours une région très importante du devenir humain qui ne peut être saisie par une analyse rationnelle. Nous pensons à cette région de la relation personnelle intime où seuls, «toi et moi», peuvent être les témoins privilégiés et uniques de la signification du dépassement réciproque qui nous lie. Il n'est donc pas possible de construire une théorie universelle de l'amour.

L'analyse philosophique est ainsi dépassée par l'unicité et la densité de l'existence individuelle et inter-personnelle des êtres concrets. C'est pourquoi, comme dit Jung, on ne peut en avoir une connaissance, mais seulement une compréhension. Pour comprendre l'homme individuel, «je dois laisser de côté toute la connaissance scientifique que j'ai de l'homme moyen, renoncer à toute théorie pour m'ouvrir, autant que faire se peut, à une confrontation nouvelle et sans préjugé»".

La philosophie reconnaît donc qu'il y a une région de l'être individuel où la raison ne peut s'introduire sans trahir aussitôt l'être qu'elle tente de décrire et de maîtriser. C'est cette région de nous-mêmes qui nous échappe, parce qu'il n'y a en elle que pur devenir, et qui resterait éternellement extérieure à nous-mêmes si l'appel d'autrui ne venait pas nous aider à la reconnaître. «Nous courons vers nous-mêmes", écrit Sartre, «et nous sommes, de ce fait, l'être qui ne peut pas se rejoindre» ${ }^{10}$. Mais il est possible, par contre, qu'autrui soit capable de venir «nous» rejoindre ou, comme dit Jung, de "nous» comprendre, non pas pour mettre fin à cette course, ce qui signifierait notre propre mort, mais pour lui donner avec nous une orientation, un sens, et ainsi l'arracher à l'absurde. C'est par l'obligation qu' autrui s'introduit dans notre propre devenir. C'est par elle qu'il contribue à notre

8 Max Scheler, op. cit., p. 107

9 C. G. Jung, Présent et avenir, p. 19

10 Jean-Paul Sartre, L'Etre et le néant, p. 253 
propre devenir et c'est en lui répondant dans nos actes que nous échappons à l'absence de sens, et pouvons tenter de chercher le salut.

C'est pourquoi, lorsque nous parlons d'obligation envers soi-même, nous ne pensons pas à une «auto-obligation», un appel que nous nous lancerions à nous-mêmes, comme un défi, à partir d'une extériorité inaccessible. Pas plus que nous ne pouvons être extérieur à nous-mêmes, nous ne pouvons nous passer d'autrui pour assurer notre devenir. Il n'y a pas d'obligation dans laquelle autrui ne serait pas impliqué. Max Scheler le souligne: "Lorsqu' on dit: "je m"oblige à faire ceci', on veut simplement dire qu'on admet un devoir à accomplir en faveur d'autrui (qu'il nous concerne nous-mêmes ou autrui)»". L'obligation envers soi-même n'est donc rien d'autre que la prise de conscience de ce qui nous concerne dans notre devoir envers autrui, et qui nous met avec lui en relation de personnes. Cela implique que nous devons nous comprendre nous-mêmes comme personnes, que nous devons accepter la réalité de notre devenir.

Bien plus, et c'est en cela précisément que l'obligation en tant que telle nous concerne. Nous avons l'obligation envers nous-mêmes de nous accepter comme l'autrui des autres, comme l'auteur de l'appel qu'ils entendent. Nous devons accepter non seulement de ne pas être la finalité de notre propre vouloir, mais de devenir celle des autres. En d'autres mots, nous prenons conscience que nous sommes non seulement la source de l'amour que nous avons pour autrui, mais que nous sommes également l'objet de son amour. C'est l'obligation de nous savoir aimables. Etre une personne, reconnaitre notre obligation envers la personne, c'est nous accepter comme existence inachevée, comme existence dépendante et insatisfaisante pour nous-mêmes, afin de la tenir pour relative, de la mettre en question pour en faire l'objet d'un devenir.

Il y a deux manières, croyons-nous, de mettre son existence en question et par là d'achever, dans un certain sens, son existence. Il y a d'abord le suicide, qui est la négation de l'existence, son refus. Mise en question irrévocable de l'existence, le suicide n'apporte cependant au sujet ni signification, ni aucune réponse à ses questions. Camus a donc raison d'écrire, au début de son Mythe de Sisyphe, que la question du suicide est la question philosophique fondamentale, car c'est par elle que l'homme décide ou non de donner un sens à une existence qui ne semble pas en avoir par elle-même. Mais choisir le suicide, c'est également renoncer à la liberté, c'est-à-dire à la possibilité d'être homme, ou du moins de devenir homme. En ce sens, il est vrai que l'homme est libre de tout, y compris de l'humain. Le suicide est une négation de l'homme, mais il est aussi l'ultime défi qui s'offre à notre liberté.

11 Max Scheler, op. cit., p. 227 
Mais il n'est pas nécessaire de supprimer l'existence pour la maîtriser et dépasser ses limites. On peut la mettre en question en y renonçant dans sa forme donnée et en la dépassant par le devenir. Certes il s'agit dans un certain sens d'une mort, mais cette "mort à soi-même», dont parle l'apôtre Paul, est d'une autre nature, elle est une transformation de l'existence en un être nouveau. Cette deuxième manière de mettre en question l'existence est ce que nous appelons l'obligation. L'appel du devenir rejette l'existence donnée pour lui donner une signification dans un être nouveau.

C'est donc par l'obligation que nous sommes appelés à être «en dehors du néant» en tant que personne. Le néant est cette vie humaine qui se contente d'être une existence parmi les choses. L'obligation qui nous sort de ce néant nous aide à mettre fin à l'existence de l'homme comme chose, pour en faire une personne. C'est par elle que nous échappons à nos rôles et à nos fonctions pour devenir un être en soi qui n'a de sens que par luimême. C'est donc aussi par l'obligation que nous pouvons mettre un terme à la négation de la personne que constitue l'éthique de la prospérité.

\section{Nous appelons personne cet être de l'homme qui existe avec un sens.}

Etre n'est donc pas autre chose que de devenir une personne. Le devenir moral ne peut être que le devenir des personnes, non le devenir du monde. Le progrès ne peut être qu'un progrès des personnes, non des choses. Il n'y a de devenir moral dans l'histoire qu'à travers des personnes. Ce sont les personnes qui, par le truchement du devenir de leur propre être, imposent une valeur morale au devenir historique, et ce n'est que dans la mesure où le devenir historique contribue au devenir des personnes qu'il peut acquérir une valeur morale. Il n'y a donc pas de croissance valable de la richesse si ce n'est la richesse non quantifiable des personnes et pour les personnes. Mais, comme tout devenir dans le monde des choses, l'histoire n'a pas de sens si les personnes ne sont pas présentes pour l'assumer et lui garantir son être.

\section{L'obligation envers la personne}

L'analyse existentielle de la morale nous conduit à expliciter cette réalité que nous nommons la personne, à en faire le centre de notre éthique de l'obligation, et à trouver dans cette explicitation les arguments de reconstruction de la morale sociale.

Disons tout de suite que nous devons situer la personne dans le devenir de l'être pour éviter deux écueils de la pensée. D'abord celui qui consiste à établir dans un être donné les perfections de la personne et à faire d'elle l'objet d'une connaissance de la métaphysique. Ensuite celui contre lequel 
bute la perspective de l'objectivité scientifique, qui consiste à limiter la personne au personnage, c'est-à-dire à cet ensemble de déterminations physiologiques, psychologiques, fonctionnelles et de comportement social, (ou le caractère, disait Max Scheler) qui se manifeste dans l'horizon restreint d'une sensibilité empirique que nous avons appelé la subjectivité épidermique.

Faire de la personne l'objet d'une connaissance métaphysique, c'est considérer le devenir du moi, qui naît de la prise de conscience de l'absurdité de l'existence, comme la simple découverte passive d'un être donné dès le départ mais qui serait resté voilé aux yeux de la conscience. La conquête de soi, qui est une authentique création de soi-même par soi-même, est réduite dès lors à une connaissance, à la saisie d'un objet par la raison; elle fait donc du «moi» l'objet de cette connaissance, ce qui revient à nier la personne. Certes, même si cet objet n'est pas celui de la science ou du monde matériel qui nous entoure, mais celui que la métaphysique tente de saisir dans la sphère pure de l'être, il n'en reste pas moins un objet et empêche pratiquement toute conscience de soi authentique. La personne ne peut être donnée d'avance, sinon elle nierait à la fois le dynamisme de l'être et la possibilité de la liberté. C'est pourquoi nous dirons qu'elle ne peut être saisie existentiellement, - Jung dirait «comprise» - que dans la conscience d'un devenir créateur, dans l'expérience même de l'action. Elle se trouve dès lors dans la condition suggérée par Sartre quand il écrit: «L'existentialisme ne prendra jamais l'homme comme fin, car il est toujours à faire» ${ }^{12}$. Il n'est donc pas possible de décrire la personne, car elle est toujours à inventer, et à inventer par chacun dans chaque choix que requiert l'action. Il n'y a donc pas de «notion de la personne», il n'y a que des personnes concrètes qui se saisissent et se «comprennent» elles-mêmes comme telles.

C'est pourquoi il n'est pas possible non plus de trouver dans les connaissances scientifiques des indications pouvant conduire notre recherche de la personne. Pour la science, en effet, la personne n'est que le point de rencontre et de concentration d'influences multiples trop nombreuses pour que les moyens actuels des connaissances puissent en faire un inventaire complet. La science, - et particulièrement la science économique qui l'ignore complètement - parle de la personne en reconnaissant qu'elle est une réalité qui se trouve au-delà de ce qui est actuellement connaissable: «Ce rien au-delà de tout ce que l'on a». Mais si elle est inconnaissable, ce n'est pas parce qu'elle se trouverait «en fait» au-delà du connaissable, mais parce que la connaissance est «en deçà» de ce qui peut être saisi ou compris du mystère de l'être. Il suffirait, pour satisfaire la science, qu'elle puisse énumérer, cataloguer, expliciter et décrire toutes les influences subies par un individu, afin de rendre compte de toutes les particularités de l'individu,

12 Jean-Paul Sartre, L'existentialisme est un humanisme, p. 92 
et par là expliquer la signification de ses actes, et ainsi les prédire. Or ce que la science ne peut retenir dans ses prémisses, c'est que l'homme n'est pas seulement l'objet d'une connaissance, mais le sujet même de cette connaissance. L'homme, à travers la connaissance qu'il peut avoir de luimême, y compris la connaissance de type scientifique, se découvre toujours comme autre, comme conscience au-delà du connaître. C'est pourquoi toute connaissance, et en particulier la connaissance de l'être humain, si objective qu'elle puisse être, ne réduit pas l'être à l'objet, mais est l'occasion d'une transformation du sujet. "Lorsque nous savons ce que nous sommes», écrit D. Parodi, "nous ne sommes déjà plus ce que nous étions» ${ }^{13}$. Le phénomène de la connaissance scientifique de l'homme conduit donc également à affirmer la personne comme un devenir, auquel la science contribue ellemême par sa nature et son pouvoir.

C'est pourquoi il est évident que l'obligation la plus fondamentale envers la personne est de prendre conscience de soi comme authenticité. Cela signifie que la personne ne peut être approchée que par elle-même, dans une communication correspondant exactement avec elle-même, et qu'elle ne peut se comprendre que dans son propre dynamisme créateur. Être authentique, en effet, c'est ne pouvoir être dépendant que de soi-même, ne pas trouver en dehors de l'être individuel les raisons de ce qu'il est et de ce qu'il fait. "On tourne le dos à l'authenticité», «écrit Georges Bastide, "toutes les fois qu'on veut se la donner en spectacle à soi-même» ${ }^{14}$. Il est donc impossible, pour la personne, de sortir d'elle-même afin de s'observer. Si nous avons reconnu que la connaissance de soi, à laquelle nous invitait la recherche socratique, qui répondait elle-même à l'injonction de Delphes, pouvait être considérée comme la première démarche de la pensée vers la prise de conscience de l'obligation, nous devons reconnaître à nouveau que cette connaissance ne peut être qu'une "conscience de soi», une saisie directe de la personne par elle-même dans son action. L'authenticité implique que l'obligation de se connaître est essentiellement morale, et qu'elle ne peut être séparée du devenir même de la personne.

La prise de conscience du sujet comme personne s'exprime le plus clairement par le terme de vocation. La vocation peut être considérée comme l'expression d'une obligation individuelle orientée directement vers l'épanouissement de la personne. C'est la découverte, par l'individu, de l'accomplissement qui lui convient, ou de la perfection de son être. C'est une obligation qui m'appelle à devenir ce que je peux, justement moi seul, devenir. Max Scheler a souligné que l'existence d'une obligation person-

13 D. Parodi, Le problème moral et la pensée contemporaine, p. 77

14 Georges Bastide, Méditations pour une éthique de la personne, p. $87 \mathrm{~g}$ 
nelle, c'est-à-dire d'une obligation qui ne peut être que "mon" obligation, se fondait sur l'expérience d'une conscience de ma personne, ce qu'il appelle «l'essence axiologique individuelle d'une personne», qu'il désigne également par l'expression: «salut personnel». Or, souligne Scheler, ce n'est pas par cette obligation que je peux me découvrir et saisir la nature de ce salut, mais au contraire en reconnaissant que "précisément cette expérience-vécue d'une obligation qui est mon obligation ... se fonde elle même déjà sur l'expérience de mon essence-axiologique individuelle»" ${ }^{15}$. Pour Max Scheler, qui refuse que la valeur soit fondée sur l'existence du devoir, mais qui au contraire voit dans l'intuition axiologique le fondement même de toute obligation morale, il est normal de dire que la saisie, par expérience-vécue, de l'essence axiologique individuelle précède l'expérience d'une obligation qui soit individuelle.

Mais il nous semble au contraire que cette antériorité logique que défend la position de Scheler n'est plus nécessaire au moment où l'on exprime le rapport d'une obligation personnelle avec la saisie de la personne par la notion de vocation. Car la vocation n'est pas autre chose que l'appel qui conduit à la réalisation de la personne elle-même et par elle-même. Elle est en fait l'obligation concrète de l'authenticité. Elle respecte totalement la personne comme un devenir puisqu'elle situe la prise de conscience de la personne dans l'appel même par lequel elle s'oblige. En d'autres mots, la vocation est l'obligation qui me demande de devenir une personne à ma propre façon, de remplir «mon devoir» par la réalisation de moi-même, et de faire de ce que je suis ce que je peux devenir.

Finalement, la personne implique une obligation d'amour envers nousmêmes. Nous approfondirons plus loin ce qu'implique l'obligation envers autrui, mais nous comprenons dès maintenant que si l'amour se fonde dans la personne d'autrui, c'est justement parce qu'autrui est une personne, au même titre que moi-même. Si donc je m'affirme comme personne, je suis également une personne aimable pour autrui, donc à ce titre même aimable pour moi-même. C'est justement parce que je suis une personne que je dois m'aimer.

Le problème de l'amour de soi est complexe et peut paraître difficile à comprendre. L'amour, à première vue, ne peut avoir de sens s'il n'existe pas un objet de l'amour. L'amour est visée, intention, tendance, finalité, et l'objet qu'il poursuit pour s'y perdre en est justement le fondement. Or si nous affirmons que la personne est l'être qui ne peut sortir de sa subjectivité, il ne peut devenir un objet pour lui-même. L'amour de soi serait donc un amour sans objet. Il y a là une contradiction dans les termes qu'il faut expliciter.

15 Max Scheler, op. cit., p. $489-490$ 
Il ne sufit pas, pour cela, de distinguer entre l'amour "éros», qui est fondé sur l'existence d'un objet désirable, qui attire et détermine la volonté, et l'amour «agapè», sur lequel se fonde une communauté dans laquelle chacun est respecté pour lui-même et peut s'épanouir à travers les autres. En effet, la personne ne peut ni devenir un objet désirable pour elle-même sans renoncer à sa nature de personne, ni constituer une communauté à elle seule. Dans son être, la personne est toujours seule. L'amour de soi ne sera donc pas autre chose que la prise de conscience de se savoir aimable pour autrui. Or nous ne pouvons prendre conscience de notre amabilité que par l'expérience vécue de l'amour réel qu'autrui nous témoigne. Il n'est donc possible de s'aimer que par le truchement d'autrui. L'homme seul est incapable d'amour pour lui-même.

Est-il dès lors possible de parler en ce cas d'une obligation d'amour envers soi-même? N'est-il pas plus logique de parler d'une obligation d'autrui envers moi, que de moi envers moi-même? Quand nous disons que l'amour de soi est la conscience de se savoir aimable pour autrui, nous parlons d'une obligation envers soi-même par la médiation d'autrui. En d'autres mots, l'obligation de s'aimer soi-même n'est pas autre chose que l'obligation d'accepter l'amour d'autrui, de s'ouvrir à l'amour d'autrui, de s'accepter comme une existence limitée et inachevée qui ne recevra sa plénitude que d'autrui. S'aimer signifie, en ce sens, accepter d'être aimé. C'est se considérer soi-même comme digne de l'amour d'autrui. Celui qui s'aime manifeste ainsi la conscience de sa propre valeur de personne. S'aimer soi-même devient alors, également, une obligation envers autrui. En effet, en nous reconnaissant nous-mêmes comme des êtres aimables, nous offrons à autrui l'occasion de se dépasser dans son amour pour nous.

Dans cette perspective nous comprenons que ne pas s'aimer soi-même est une forme d'égoïsme. L'égoïsme peut en effet prendre deux formes particulières. D'abord ce peut être un refus de l'amour d'autrui, un orgueil qui consiste à rejeter l'obligation de se reconnaître comme aimable, ou, si l'on veut, à croire que l'on peut s'accomplir soi-même sans la valorisation que nous apporte l'amour d'autrui. C'est une forme très courante d'égoïsme. L'expérience montre qu'il est plus facile de prodiguer son amour à l'autre que d'accepter d'être aimé. L'homme trouve plus satisfaisant de se savoir généreux que de recevoir par amour.

La deuxième forme de l'égoösme consiste à faire de soi-même l'objet d'un amour «éros", et de se transformer soi-même en finalité de notre propre désir. Mais en s'objectivant par le désir, l'homme se désintègre comme personne, il abandonne sa nature de sujet, s'établit dans l'être accompli et renonce à sa vocation. C'est un refus flagrant de l'obligation envers son être propre. Tous les «narcissismes» conduisent ainsi à une forme d'autodestruction. 


\section{L'obligation envers la conscience}

Si l'obligation nous demande de prendre conscience d'une vocation qui soit l'expression du moi authentique, cette vocation s'identifie au sentiment de responsabilité. La responsabilité est ce sentiment intime par lequel on «comprend» que les manifestations de l'être dans l'action ne peuvent être rattachées qu'à l'être lui-même, celui qui se découvre dans la vocation. La responsabilité est l'empreinte de la personne sur l'action. Elle est une forme de certitude pour la personne que la conscience de soi n'est pas un simple repli réflexif sur soi-même, mais la manifestation de son authenticité aux yeux du monde.

Nous avons dit que la notion de responsabilité était l'une des implications de la subjectivité nécessaire de l'action pour être morale. Nous devons revenir sur cette notion à ce niveau de notre raisonnement, non plus pour en souligner la pertinence logique dans l'analyse des concepts moraux, mais parce qu'elle se présente comme l'exigence première de la personne concrète qui s'identifie à une vocation. Par cette responsabilité, nous «comprenons» aussi que nous parlons maintenant d'une conscience de soi qui n'est pas seulement la saisie intellectuelle d'un sujet qui se sait inobjectivable en tant que sujet dans l'ordre de la raison, mais d'une conscience existentielle engagée dans l'action par laquelle elle se crée, dans une relation valorisante avec autrui où l'amour se construit. Il n'y a donc pas de conscience possible de la personne, qui soit existentielle, autrement que par l'action et dans la moralité. La responsabilité devient donc la condition précise de l'expérience-vécue par laquelle la personne se comprend à la fois comme conscience de soi et comme conscience morale.

Le rapport entre la conscience de soi et la conscience morale ne peut être saisi que dans le devenir. Il y a donc une sorte de concomitance entre le progrès de la personne et ce que nous devons appeler la "personnalisation" progressive de ses actes. De même il y a, dans l'action, une progression de la conscience de soi et du sentiment de responsabilité, qui est le fruit du devenir de la personne. Plus l'homme progresse sur le chemin qui le conduit à sa propre personne, plus fort se manifeste le sentiment de responsabilité, à mesure que l'action et la personne elle-même deviennent plus proches et plus identiques. Une forme d'obligation envers la conscience grandit ainsi au rythme du développement de la personne, consistant à exiger plus de responsabilité et plus de conscience morale à mesure que tout l'être devient plus personnel. En d'autres termes, la conscience de soi rejoint peu à peu l'authenticité et s'identifie toujours plus à la vocation.

Mais cette conscience de soi se rapproche aussi de l'appel qui lui demande de devenir elle-même. De ce fait, répondre à l'obligation, c'est la renforcer; c'est donner plus de résonance intérieure à l'appel de l'obligation. L'obligation n'apparaît donc pas seulement comme un appel isolé dans l'espace et le temps, chronologiquement ou spatialement identifiable, qui 
exige quelque chose dans une situation précise et limitée, elle fait partie de la permanence du devenir dont la force grandit avec la personne. La conscience morale de l'obligation ouvre à la conscience de soi, de la même façon que grandir dans la conscience de soi-même favorise la croissance de la conscience morale.

C'est ainsi que la conscience morale, qui est celle du Bien et du Mal, s'identifie avec la conscience d'une personne en devenir. Nous avons dit que le Bien était la valeur d'un devenir possible de l'homme, le Mal la négation de ce devenir. Nous comprenons, maintenant, dans la perspective de l'êtrede-la-personne, que la conscience morale n'est autre chose que la conscience du devenir humain par la personnalisation du monde et des choses. La conversion morale, c'est-à-dire l'adhésion du sujet à l'appel de l'obligation et la personnalisation se situent dans le même mouvement de l'être qui conduit à la moralité. La moralité de l'action humaine progresse avec la personnalisation de l'être, et la conscience grandit à la mesure des progrès de la moralité. Finalement, conscience de soi et conscience morale apparaissent comme deux expressions, distinguées logiquement par l'analyse, qui recouvrent une seule réalité: la conscience de la personne. L'obligation morale n'exige pas davantage de «suivre» sa conscience, que «d'être» une conscience.

La conscience de soi n'est donc pas autre chose que la conscience morale. Voilà pourquoi nous disons que c'est avant tout dans la vocation personnelle que se rencontre l'obligation. Dès lors la moralité conduit à l'unité de l'être. C'est d'une part l'unité de la personne avec son propre devenir, mais c'est d'autre part l'unité de cette personne individuelle avec les autres, qui construisent ensemble une moralité dans laquelle elles vont se retrouver. On peut ainsi parler d'obligation envers soi-même et d'obligation envers autrui, mais elles ne peuvent en fait être séparées. La conscience morale de soi est aussi une conscience des autres.

Nous comprenons mieux maintenant pourquoi l'obligation apparaît comme l'approche que propose la philosophie pratique pour redonner un sens à l'individu que le monde de la prospérité rend exclusivement fonctionnel. En faisant de lui une personne qui soit pour elle-même sa propre fin, le sujet trouve dans l'afffirmation de sa responsabilité morale un lien qui, tout en lui permettant de se libérer de la contrainte sociale, le protège de l'individualisme et ne l'isole pas de ses responsabilités envers autrui. L'obligation morale est une forme de la conquête du salut. 


\section{Chapitre 5}

\section{Les illusions de l'obligation, ou la société-objet}

Il n'y a pas de liberté humaine en dehors du temps.

Pierre Chaunu La liberté

Ce n'est qu'd partir d'un certain niveau d'existence humaine que le concept-de-personne trouve son application.

Max Scheler Le formalisme en éthique

En situant l'obligation morale dans l'appel qu'autrui m'adresse pour construire avec lui la moralité, nous soulignons la dimension essentiellement sociale de cette dernière. L'idée de société, avec tout ce qu'elle implique dans le façonnement des comportements humains, trouve donc logiquement sa place dans notre analyse de l'obligation. Il ne pourrait d'ailleurs en aller autrement, car l'expérience d'autrui est premièrement, et presque toujours exclusivement sociale. C'est celle d'un autrui multiple et dense, ayant la force, la densité et l'anonymat du groupe. Autrui, ce sont d'abord les autres, tous les autres, ou du moins tous ceux qui constituent le groupe au sein duquel nous prenons conscience de nous-mêmes. Ce n'est qu'à un autre stade, dans la relation privilégiée de l'amour individuel, qu'autrui s'incarne dans une seule personne et prend le visage unique de l'être irremplaçable. 
Parler de la société, en tant que de la réalité spécifique de l'autrui moral, dans le type d'analyse de la vie morale que nous proposons, soulève aussitôt un problème. Qu'est-ce que cette société? Que représente-t-elle dans la réalité existentielle de chacun? Est-elle un sujet ontologique? Est-elle morale par nature? Faut-il l'identifier à l'objectivité intersubjective que nous avons définie comme l'objectivité spécifiquement morale, et en faire dès lors la source de la norme? Est-elle de ce fait porteuse d'une obligation fondée en dehors des sujets, dans une réalité préétablie qui s'impose à la conscience morale, et au service de laquelle les sujets seraient prédestinés et soumis, au prix de leur autonomie? Allons-nous tomber à notre tour dans le piège de Durkheim, et accepter une nouvelle forme d'ontologisme social sans lequel il ne serait plus possible d'expliquer l'obligation?

Disons d'emblée que nous ne pouvons pas éviter, dans l'élaboration progressive de l'anthropologie morale à laquelle notre analyse nous conduit, d'aborder de front le problème des relations entre sujets et société, et par là entre obligation et société. Si la société est le premier autrui moral dont nous faisons l'expérience, cet autrui doit être sujet pour être source de l'appel qu'il me lance. Il faut dès lors poser le problème de la société comme celui d'un possible sujet, appelé lui aussi par une obligation qui lui est propre. Ce problème est complexe et doit être discuté.

La complexité sociale ne se laisse pas toujours réduire à la seule approche philosophique. Nous croyons cependant que la méthodologie poursuivie dans ce travail nous autorise à entreprendre une telle analyse, mais dans une perspective spécifiquement morale et pratique, c'est-à-dire, - pour rester fidèle aux exigences de l'obligation - dans une perspective d'invention. Nous savons que ce n'est pas dans l'objectivité du monde empirique que nous devons chercher les éléments essentiels de la moralité. Cette dernière, en effet, ne peut recevoir son existence réelle que de la libre volonté des sujets qui en sont à la fois les créateurs et la forme. Il n'est donc pas possible d'aborder l'étude des relations entre sujets et société à partir d'une réalité sociale de fait qui serait l'objet de la science. Une telle objectivité ne serait plus d'ordre moral.

La science, elle, est limitée par ses propres hypothèses. Elle ne peut accepter un devenir qui ne fasse pas l'objet d'une prévision, c'est-à-dire un devenir qui ne soit pas déjà inscrit dans le constitué. La science ne peut tenir compte de l'imprévisibilité qui résulte de l'engagement du sujet dans son acte. La science a besoin, comme le dit George Bastide, d'un corps constitué de vérité. "Or, la vie morale que l'on voudrait faire sortir de la science est une action constituante de moralité. Les deux choses ne sont pas du même ordre»'. Certes, nous ne mettons pas en doute que la société est un fait, donné, indéniable. Mais le regard que nous posons sur elle nous permet de la reconstituer, pour ainsi dire, dans une perspective morale.

${ }^{1}$ Georges Bastide, Traité de l'action morale, p. 334-335 
Aborder la société dans une perspective morale implique que la société, comme l'action du sujet et la moralité, doit être imprégnée des deux dimensions de la morale, d'une forme dans laquelle la subjectivité des agents se retrouve, et d'une objectivité qui implique un jugement de valeur, à l'aune d'une norme également de nature morale. Comme la morale, la société humaine est ambiguë.

Ceci a pour conséquence que nous devons postuler, non pas dans l'être mais dans le devenir, une société qui ait, du point de vue de la philosophie pratique, les caractéristiques d'un sujet moral. Ceci consiste tout d'abord, par rapport à l'action du moi, à reconnaître dans la société un sujet-autrui, qui se présente comme la réalité symbolique des autres «je» que sont les sujets-autrui, au nom de qui elle m'appelle et me propose une obligation. L'appel d'autrui dans lequel nous avons finalement ancré l'obligation ne se présenterait donc plus comme une multitude d'appels individuels, mais comme un appel commun venant d' un sujet moral global, la société, reconnu comme mon autrui.

Or, pour que cette reconnaissance puisse avoir lieu, c'est-à-dire pour que notre postulat $n$ 'apparaisse pas comme un simple artifice logique, il faut montrer tout d'abord en quoi la société n'est pas qu'une force objective et empirique d'action sur l'individu. Si elle n'était que cela, elle ne pourrait être source d'obligation, car elle ne pourrait assumer de responsabilité et porter un jugement de valeur comme peut le faire un autrui-sujet. En d'autres mots nous dirons que quand la société n'invente pas, elle n'oblige pas, elle contraint. Voilà pourquoi nous disons, et c'est ce que nous souhaitons montrer dans ce chapitre, que l'action accomplie sous la pression objective de la société n'est qu'une illusion de l'obligation. Il n'y a pas de véritable obligation morale venant d'une «société-objet». Nous plaçons donc la moralité au-dessus, ou du moins en dehors du social empirique.

L'intention qui anime tout ce livre - redonner une place centrale au sujet responsable devant les déviances sociales de l'éthique de la prospérité - doit également inspirer notre approche du phénomène social. Sinon notre démarche apparaîtrait comme une tentation de s'abandonner à l'individualisme le plus extrême, et nierait toute possibilité de transformation de la société. Pour cela, la première obligation de la recherche morale est de considérer la société-sujet que nous postulons d'un point de vue normatif. Il ne suffit pas de la décrire telle qu'elle est et d'en révéler le fonctionnement intérieur, mais de montrer en quoi elle peut être un idéal. Or c'est la nature même de l'idéal d'être toujours situé au-delà du réalisable, au-delà de l'achevé. C'est pourquoi la société dont nous parlons ici, en tant que société morale, est toujours une société inachevée, une société à faire; elle est comme le sujet moral: un devoir être. En affirmant cela, nous suggérons de voir en elle le résultat de l'intersubjectivité morale plutôt que son fondement. C'est pourquoi notre postulat n'est pas un artifice ontologique, tel le postulat durkheimien, mais un postulat moral qui fait de la société-sujet une réalité à constituer 
moralement par l'interaction des sujets. Elle entre dès lors dans l'histoire et est soumise au temps. L'obligation évite ainsi d'enfermer le sujet dans le solipsisme moral.

Pour y parvenir, il faut d'abord montrer en quoi le façonnement des comportements humains par la société n'a rien à voir avec l'obligation. En effet, sans nier l'existence d'une société-objet qui est faite avant tout de déterminismes sociaux que la sociologie met en évidence, nous ne pouvons réduire l'action humaine à un enchaînement inéluctable de causes sociales, puisque c'est justement pour en libérer l'homme que nous faisons appel à l'obligation. Or, comme nous avons reconnu que la connaissance de la nature humaine pouvait aider le sujet à se libérer, du moins partiellement, de déterminismes qui étaientà l'origine d'une subjectivité illusoire, épidermique et irréponsable, il y a une connaissance de la socialité humaine qui peut efficacement contribuer à faire découvrir l'essence réelle de ce que nous appelons ici la société morale ou société-sujet, celle qui se présente comme un idéal dans lequel le sujet n'est pas renié. Pour le faire, nous devons montrer en quoi certaines exigences de cette socialité ne constituent pas des obligations authentiques, mais des illusions de l'obligation. 11 s'agit de les démasquer afin d'atteindre l'obligation réelle.

\section{La notion de contrainte}

Il est inutile de mentionner les déterminismes sociaux parmi les illusions de l'obligation puisque, en raison même de leur ignorance du sujet, ils ne laissent aucune place à la liberté et ne sont donc pas d'ordre moral. Il en est tout différemment de la contrainte sociale.

La notion de contrainte implique que les êtres qui lui sont soumis ne sont pas déterminés à faire ce qu'elle demande. Etre contraint signifie pour l'homme d'être poussé à des actions auxquelles il n'est onduit par aucun déterminisme naturel. Entendre ou voir ne sont pas une contrainte puisque l'ouîe et la vue sont des déterminations essentielles de la structure de notre sensibilité. Nous devenons conscients d'une contrainte quand, dans une situation particulière, certaines exigences vont à l'encontre d'une tendance naturelle ou désirée, ou quand ces exigences s'opposent directement à notre liberté. Il y a contrainte quand l'homme n'est pas en état de satisfaire ses appétits fondamentaux (manque de nourriture, par exemple); il y a contrainte aussi quand l'homme ne peut réaliser ses désirs ou ses rêves, soit parce qu'un obstacle se trouve sur son chemin (l'existence d'une frontière), soit parce qu'une limitation de sa nature l'en empêche. Ce n'est pas d'aujourd' hui que l'impossibilité naturelle de voler comme les oiseaux est ressentie comme une contrainte par de nombreux Icare ${ }^{2}$. Et il y a surtout contrainte quand la liberté est limitée, quand la volonté d'agir est mise en échec (emprisonnement, censure, etc.) 
Ce qui caractérise principalement la contrainte, et la distingue essentiellement des déterminations, est le fait qu'elle suppose, par définition, la liberté. Du moins elle suppose la possibilité d'autres actions et le choix d'autres buts. Nous savons que nous sommes contraints parce que nous nous savons libres. Si, comme l'obligation, la contrainte suppose la liberté, nous sommes en droit de nous demander pourquoi la contrainte et l'obligation ne sont pas, en fait, de même nature et ne remplissent pas la même fonction. La contrainte n'est-elle pas une forme d'obligation inscrite ou manifestée dans notre nature et notre condition d'être social?

Une réflexion plus approfondie est nécessaire pour répondre à cette question. Certes, il existe des contraintes d'ordre métaphysique qui ne contraignent que ceux qu'elles révoltent. Il y a aussi des contraintes physiques, que nous avons parfois vaincues par l'invention technique (voler, par exemple) qui ne posent pas de problèmes particuliers à la réflexion morale, car elles ne concernent pas directement l'agir humain. Mais nous devons analyser plus profondément les contraintes que nous ressentons comme directement d'ordre moral.

\section{Les contraintes individuelles}

La contrainte apparaît d'abord comme une limitation, comme une entrave à la liberté d'action d'un sujet particulier, donc comme une limitation de sa responsabilité. Elle peut venir, soit du dehors, soit du dedans.

La contrainte externe consiste à empêcher un individu d'agir librement, soit en lui imposant des entraves physiques, (emprisonnement) soit en le conditionnant psychologiquement de telle façon que sa capacité de décision et sa responsabilité soient partiellement diminuées ou même totalement supprimées. Il n'est plus possible dans ce cas de parler de liberté réelle, d'action morale ou de responsabilité. On n'impute pas d'actes libres à un esclave enchaîné. Certes, bien que son caractère de contrainte suppose l'existence d'une liberté, cette dernière est tellement obnubilée par les limitations extérieures qu'elle en est pratiquement niée. Il n'est donc pas possible de parler d'action réellement morale, donc d'une obligation qui s'y rattache.

2 Certes l'homme ne vole pas comme un oiseau, mais il a inventé des substituts techniques qui l'arrachent de la surface du sol, et même le libèrent de la planète. Par la technologie, l'homme relativise la contrainte du corps, et de ce fait «moralise» l'action qui en découle. En effet, les contraintes vaincues par l'invention technique sont des contraintes de l'être modifiées par l'action. Or l'action par laquelle l'homme modifie le fonctionnement de son être est directement morale. 
Une limitation similaire de la liberté naît également de la contrainte interne. C'est généralement une contrainte par défaut qui conditionne l'individu souffrant de déficiences mentales ou physiques qui affectent visiblement sa responsabilité. C'est la contrainte des "maladies de l'âme», selon l'expression de Max Scheler. Le manque caractérise avant tout la contrainte interne, et non un obstacle ou une opposition. La contrainte interne est l'absence de quelque chose qui devrait être. En ce sens elle suppose également la liberté, car on ne prend conscience de cette contrainte qu'en faisant référence à cette liberté en défaut, ou à une autonomie de la volonté qui demeure supposée malgré les déficiences qu'elle connaît dans ses manifestations. Scheler le reconnaît quand il écrit: «Il y a des maladiesde-l'âme, il n'y a aucune maladie-de-la-personne». ${ }^{3}$ La contrainte interne se définit donc par rapport à des facultés qui manquent, par rapport à une liberté attendue qui ne se manifeste pas. L'action accomplie sous l'emprise d'une telle contrainte n'est donc pas soumise à la responsabilité de l'agent et elle ne peut lui être imputée. Dans ce cas elle est totalement a-morale, et sans rapport possible avec l'obligation.

Ces deux formes de contrainte ramènent l'action au niveau de ces «actes de l'homme» qui sont a-moraux et sans engagement du sujet, qui ne peuvent donc avoir aucun lien quelconque avec l'obligation morale.

Certes, les actions faites sous l'influence de telles contraintes, particulièrement les contraintes externes, ne peuvent être assimilées à des conduites soumises entièrement au déterminisme. Elles connaissent en effet une différence psychologique notoire dans le fait que, même si elles ne sont pas libres, elles peuvent être conscientes. Le sujet peut savoir ce qu'il est contraint de faire dans une situation donnée sans pouvoir y résister, sans vouloir réellement cette action, donc sans y engager sa liberté. II n'en assume pas l'intention propre. Ce sont des actions dont nous avons dit qu'elles pouvaient être justifiées. ${ }^{4} \mathrm{La}$ finalité est connue de l'agent, mais pas voulue par lui. Que la contrainte soit, dans ce cas, externe ou interne, physique ou psychologique, le résultat est le même. La contrainte se définit par une absence de liberté, par la négation de ce qui fait formellement un acte pleinement moral. Comment donc concilier cette contrainte avec une obligation dont nous avons dit qu'elle obligeait pour rendre libre?

\section{La contrainte sociale}

La question est différente par rapport à une autre forme de contrainte, celle que ressent tout individu du fait qu'il vit en société. C'est un problème

3 Max Scheler, Le formalisme en éthique, p. 487

4 Voir plus haut p. 119 
beaucoup plus complexe, dans lequel l'action humaine est plus influencée que contrainte. Il est dès lors plus difficile de distinguer cette situation d'une véritable obligation.

Toute société, en effet, exige que les individus qui la composent se conforment à certaines normes de conduites, à certaines conventions de «moralité sociale» sans lesquelles la vie en société serait impossible. Cette «moralité sociale» varie d'une société à l'autre - l'éthique de la prospérité en est une en particulier - et de ce fait elle ne peut prétendre à l'universalité. Mais il n'en demeure pas moins vrai que c'est cette moralité sociale, faite de moeurs, de coutumes, de lois et de codes, qui définit la société et en assure en même temps l'existence. Or, cette moralité imposée joue en fait un double rôle. D'une part elle s'impose à chaque individu, pris individuellement, mais aussi à tous les individus, pris collectivement. La moralité sociale n'a pas pour seule fonction de socialiser l'individu, mais aussi de garantir la survie de la société. Il y a donc un fonctionnalisme de la contrainte sociale qui est indéniable. Une première question se pose dès lors: peut-on assimiler cette contrainte sociale à une cause finale de l'action?

Nous avons vu, dans le Chapitre précédent, comment la moralité pouvait être considérée comme la cause finale de l'action morale. Nous devons voir maintenant si, en introduisant la notion de société comme but de la moralité, nous ne lui reconnaissons pas la fonction de finalité ultime, au-delà de la moralité elle-même. En effet, la société a besoin de la moralité comme fondement. Mais, dans une telle logique, par la moralité dont ils ont besoin pour être, les sujets deviendraient eux-mêmes les instruments de l'actualisation de la société. La finalité de l'action morale ne serait donc plus la construction de la moralité, mais celle de la société. Cette moralité étant reconnue comme une fonction sociale, nous devrions dire que la contrainte sociale est une contrainte authentiquement morale. Il y aurait donc une contrainte sociale réellement morale, ayant la forme d'une obligation, fondée sur l'existence de la société, par laquelle cette société serait capable d'imposer aux sujets les conduites particulières nécessaires à l'existence sociale elle-même. Nous avons tenté de réfuter cette thèse lors de nos analyses des travaux de Durkheim et de Bergson. Nous devons cependant y revenir à ce stade de notre réflexion.

Cette contrainte sociale, en effet, contrairement aux autres contraintes que nous avons mentionnées plus haut, ne se définit pas par la suppression ou la limitation de la liberté qu'elle présuppose, mais elle se fonde également sur l'existence de cette liberté qu'elle tente d'orienter vers une fin sociale donnée. Les individus ne manifestant pas de tendance naturelle à assurer la moralité sociale, la société développe une contrainte dans laquelle elle propose la société comme finalité désirable. Dans une certaine mesure, la contrainte sociale supplée aux déficiences des individus qui, étant libres, ne sont pas déterminés à vouloir cette finalité sociale. La contrainte sociale ne crée donc pas un manque de liberté, mais elle est là pour remplacer ce manque. Elle n'est pas négative, comme la contrainte par déficience ou par 
obstacle, mais elle soutient et oriente positivement l'action des individus vers l'actualisation d'une fin: la société. Il semble donc que l'on y retrouve tous les ingrédients qui en font une authentique obligation morale.

Pour le savoir de façon définitive, et voir dans quelle mesure cette contrainte sociale est une forme d'obligation ou une illusion de celle-ci, nous distinguerons deux sortes de contraintes sociales, deux manifestations différentes de la force contraignante de la société: la contrainte sociale organisée, qui se fonde sur les lois, règlements, statuts, codes moraux rationalisés, et la contrainte sociale diffuse, qui s'appuie sur les moeurs, coutumes, tabous, normes morales non rationalisées. C'est par rapport à chacune de ces deux formes de la contrainte sociale que nous devons tenter de dégager ce qui se rapproche ou se distingue de l'obligation morale telle que nous l'avons jusqu'ici révélée.

\section{La contrainte sociale organisée}

Elle est fondée sur des lois, règlements, codes rationalisés de moralité sociale, constitutions, etc. Elle implique donc l'existence d'une structure capable de légiférer et d'imposer ses lois, donc d'un Etat. Aborder l'analyse de la contrainte sociale par cette forme particulière peut paraître à première vue aller à l'inverse d'une chronologie inscrite dans les faits qui correspond mieux à l'évolution historique des sociétés. Il est évident, en effet, que les coutumes, moeurs et tabous préexistent dans la vie morale des individus aux lois qui les prescrivent comme normes légales de comportement. Il semblerait donc plus logique de s'attacher d'abord à leur analyse, et chercher le fondement de la contrainte organisée dans les éléments sur lesquels s'appuie la contrainte diffuse. Nous croyons cependant qu'il ne faut pas confondre la chronologie historique des faits et la chronologie logique de leur révélation et de leur compréhension. Dans les sociétés dites primitives, il n'y a apparemment pas de différence entre la morale diffuse des moeurs et la morale juridique des lois. La différence entre les deux contraintes, qu'il est facile d'établir dans l'étude des sociétés contemporaines, n'est donc pas possible. C'est parce que l'évolution des sociétés a conduit les pouvoirs responsables de ces sociétés, corps constitués, Eglises, organisations, communautés volontaires et Etats, à codifier certains aspects de la «moralité sociale», à rédiger sous forme de lois certains contenus de normes morales, que nous sommes capables de distinguer ce qui ressortit à cette morale rationnellement organisée et ce qui est resté du ressort de la morale diffuse. Cette distinction ne nie pas que la morale, que nous pouvons appeler légalisée, trouve en partie son fondement dans l'existence d'une morale diffuse non-légalisée, mais il n'aurait pas été possible de prendre conscience d'une morale diffuse si la société n'avait pas organisé, sous formes de lois et de codes, certains donnés vécus de la morale sociale. Nous serons donc mieux à même de décrire et de comprendre ce que représente la contrainte sociale diffuse quand nous aurons décrit la contrainte organisée qui est plus 
facilement descriptible et abordable puisqu'elle est bien délimitée par ses règles propres et qu'elle est entièrement soumise aux lois manifestes qui la régissent.

Quand la contrainte sociale est organisée, c'est-à-dire quand elle manifeste explicitement les fins que l'action individuelle doit poursuivre pour réaliser la moralité sociale sous formes de lois, réglements et codes, le sujet est respecté en tant que sujet conscient et responsable. En effet, si le groupe voit la nécessité de publier ouvertement ce qui doit être fait, s'il manifeste ses intentions et les communique, («nul n'est censé ignorer la loi»), c'est qu'il respecte en quelque sorte la décision du sujet et tient à ce qu'il soit conscient de ses obligations sociales. Il y a, dans la contrainte organisée, une tendance évidente à demander la participation consciente des individus, une sorte d'appel à leur collaboration. Les lois doivent être connues de tous pour être impératives. C'est ce qu'avaient compris les Grecs, sous le législateur Solon, quand ils ont fait graver les lois de la cité sur l'agora d'Athènes. C'est ce que font les Etats modernes avec leurs publications officielles, puisqu'aucune décision des gouvernants ne peut avoir force de loi sans y avoir été préalablement publiée. C'est donc par la loi que les individus découvrent ce qu'ils doivent faire et ce qu'ils doivent éviter. En dehors de ce que spécifie la loi, et avant que cette loi ne soit publiée, les sujets ne sont pas légalement obligés.

Il faut d'emblêe noter que les lois et les codes sur lesquels s'appuie cette morale sociale juridique ne spécifient que la matérialité et les contenus de l'action morale légalement exigée. Ils définissent exclusivement le contenu du «moral» et de «l'immoral», pour reprendre les distinctions que nous avons proposêes plus haut, sans faire aucune référence à une forme morale dans laquelle serait fondée également l'obligation d'obéir à la loi. Il y a une objectivité évidente de la «norme-loi». L'aspect formel de la loi, dans les structures juridiques, n'est pas d'ordre moral; il se situe uniquement dans la légalité de la procédure par laquelle la loi a été établie. Or cette procédure est, dans la majorité des cas, également une loi elle-même, le plus souvent constitutionnelle. C'est pourquoi nous dirons que la loi de la société n'est pas «moralement» impérative, car elle laisse de côté la dimension formelle subjective.

Ce n'est pas dans la loi que le sujet trouve les raisons de lui obéir. La publication d'une loi n'assure pas par elle-même son actualisation dans l'action concrète des individus concernés. Il n'y a donc pas, contenu dans la loi, un fondement qui établit quelque obligation de la respecter. L'obligation se trouve en dehors de la loi, dans la conscience que l'individu peut avoir d'une obligation générale envers la société, obligation qui trouve sa matière dans les lois, mais qui n'est pas formellement fondée en elles. Les lois supposent l'existence d'une obligation morale, et nous reconnaissons que c'est en s'appuyant sur cette obligation qu'elles peuvent se proposer comme impératives, mais elles n'ont pas par elles-mêmes le pouvoir de fonder cette obligation. 
C'est pourquoi la société doit recourir à la contrainte, de façon légale aussi, pour atteindre son but. Puisque le sujet n'est pas subjectivement obligé d'obéir à la loi par le simple fait de l'existence de cette loi, la société se voit forcée d'établir un système de contrainte et de sanction qui s'impose aux sujets. La société ne propose pas les lois, elles les impose. Elle poursuit et punit les infractions à ces lois et, qu'il le veuille ou non, qu'il se sache obligé ou non, le sujet se trouve contraint de lui obéir. La crainte du gendarme devient ainsi le commencement de la sagesse.

Mais nous devons aussitôt reconnaître, si l'obéissance à la loi n'est fondée que sur la crainte de la sanction, que la contrainte sociale organisée a pour conséquence de supprimer, chez l'individu, toute initiative ou créativité morale. Il n'y a plus, dans la soumission à la loi, d'invention morale et de responsabilité sans lesquuelles le sujet ne peut se dépasser. Car la morale que propose la loi est une morale limitative. Dans la grande majorité des cas, en effet, les lois et règlements que la société propose sont formulés comme des interdictions. Les préceptes de la morale légale de la société contiennent davantage de garde-fous que d'idéals. La loi sociale se présente comme une contrainte non seulement parce qu'elle menace de sanctions, mais parce qu'elle propose presque exclusivement des contenus à éviter. C'est pourquoi, quand le sujet moral s'abandonne à la perspective d'une morale juridique, il perd peu à peu le sens de sa liberté créatrice et devient un instrument passif dans la construction d'une moralité dans laquelle il ne se retrouve nullement. C'est pourquoi c'est souvent la prise de conscience de cette contrainte qui est à l'origine du besoin de révolte. La révolte est l'arme du sujet conscient qui ne veut pas renoncer à la possibilité d'être lui-même par une authentique obligation.

Tenant compte du fait que, par la force de la loi, la société impose une certaine passivité des comportements, on comprend qu'une éthique dynamique, telle l'éthique de la prospérité, qui vise une progression sans limites et s'appuie sur l'esprit d'entreprise des individus pour y parvenir, tente de supprimer cette passivité et recoure pour cela à une demande généralisée de dérégulation. C'est d'ailleurs un des aspects séduisants de cette éthique de l'expansion. Mais c'est aussi son danger pour la vie sociale. En effet, si elle ne peut s'épanouir que dans la dérégulation, elle peut aussi contenir en ellemême les risques d'une destruction de la société par suppression de la contrainte sociale organisée. Cet aspect sera discuté plus loin.

Il demeure vrai que la société, en organisant et en explicitant sous formes de lois et de codes les fins qu'elle propose, respecte fondamentalement la conscience du sujet et sa responsabilité. La contrainte sociale organisée ne cherche pas seulement à imposer, elle essaie également de convaincre. Platon, dans son discours sur les Lois, le soulignait déjà.

C'est pourquoi, sans être une obligation en elle-même, et sans pouvoir se substituter à l'obligation en raison de l'absence de justification morale formelle, la contrainte sociale organisée est par contre susceptible de faire 
naître l'obligation. Il est possible, en effet, qu'un sujet, conscient de sa responsabilité et de sa liberté, puisse découvrir, dans les lois que la société lui impose, les qualités de bien, de désirable, d'idéal et d'appel à la responsabilité qui constituent la nature d'une authentique norme morale. Dès lors la loi n'apparaît pas seulement comme loi, mais comme valeur. Ce n'est pas en raison de son contenu juridique, mais dans l'éveil à la valeur qu'elle peut créer dans la conscience morale du sujet que la loi peut être l'occasion d'une réelle construction de moralité. L'obéissance à la loi ne sera plus fondée sur la crainte du gendarme, mais sur la prise de conscience que le contenu de la loi peut être, lui aussi, un authentique contenu moral de valeur. Le sujet est alors obligé, et non plus contraint, d'obéir à la loi, non pas parce qu'elle est une loi et qu'elle est sociale, mais parce que, en tant que valeur, elle est saisie comme morale. La loi est alors morale non pas parce qu'elle est l'expression d'un ordre juridique particulier, mais parce qu'elle est reconnue par le, ou les sujets, comme la manifestation d'une norme-valeur dans laquelle s'exprime la communauté intersubjective des sujets. En ce sens on peut dire que la société assume la responsabilité commune du contenu moral de la norme. C'est alors que se développe une véritable société autrui dotée de responsabilité. Il ne suffit pas de la loi pour que la moralité soit instaurée dans la vie sociale, il y faut aussi l'obligation.

Car ce n'est pas dans le texte même de la loi que le sujet trouve les éléments qui lui font comprendre qu'il est plus obligé que contraint, mais dans ce que la loi emprunte à la morale. Le changement d'attitude du sujet ne dépend pas de la matière morale, exprimée ici dans le contenu de la loi, mais dans l'intention du sujet, dans la visée d'une finalité que l'agent se donne à lui-même.

C'est donc toujours le sujet qui demeure l'authentique créateur de moralité, même si cette création implique une actualisation du contenu de la loi. L'intervention de l'intention subjective donne à la simple valeur légale de la loi une réelle valeur morale. Nous retrouvons ici la distinction essentielle que fait Kant entre les actes légaux ou juridiques, conformes au devoir, et les authentiques actes moraux, faits par devoir. En d'autres mots, les actes jugés en raison de leur seule conformité à la loi ne sont pas réellement moraux si l'intention morale du sujet ne vient pas leur donner la plénitude morale de la valeur. La moralité ne naît pas dans l'indifférence des sujets; elle exige leur participation. C'est le sujet lui-même qui demeure responsable de faire en sorte que l'objectivité sociale devienne aussi une réalité responsable de la contrainte qu'elle impose. C'est pourquoi la contrainte sociale organisée, qui ne vise qu'à obtenir des membres de la société des actes juridiques dans le but de maintenir la société, ne peut être une obligation morale pleine et entière. La morale se trouve à un autre niveau, au-delà de la simple actualisation de la société, aussi idéale que cette société puisse être conçue. Voilà pourquoi la philosophie du droit, limitée à son propre objet, ne peut donner non plus une justification suffisante de la véritable obligation morale. 


\section{La contrainte sociale diffuse}

Qu'en est-il maintenant de la contrainte sociale diffuse? Quel rôle moral et quel fondement d'obligation peuvent représenter dans la vie morale ces moeurs, coutumes non-codifiées, ces opinions communes généralement et spontanément admises, ces tabous de toutes sortes auxquels l'homme obéit presque automatiquement sans toujours savoir pourquoi?

Notre intérêt, dans ce travail, n'est pas de faire oeuvre empirique et descriptive d'anthropologue, ni de montrer comment fonctionnent les sociétés régies par la seule contrainte sociale diffuse. Notre objet est le monde actuel de l'éthique, et pas celui des sociétés dites «primitives» aux structures "élementaires» ${ }^{5}$. Or l'observation montre que la contrainte diffuse, bien qu'elle soit en grande partie remplacée par la contrainte sociale organisée, ne disparaît pas avec l'avènement des lois formelles et de la contrainte légale. Elle continue à se faire sentir dans d'immenses domaines de l'activité sociale, en dehors des lois et du formalisme juridique. Elle n'a pas seulement pour but d'orienter l'action de l'agent afin que celle-ci corresponde plus étroitement aux buts de la société, mais elle s'étend également à la réglementation de la vie individuelle. C'est pourquoi nous pensons qu'il n'est pas possible de voir dans la contrainte diffuse une forme plus primitive de la contrainte organisée et le fondement de cette dernière, même si la logique du développement social et l'expérience de la modernisation laissent supposer l'existence d'une antériorité chronologique. La contrainte diffuse est un aspect très spécifique de la vie morale qui doit être considéré comme tel.

La première chose qui doit être reconnue, c'est qu'il n'est pas possible de rattacher l'idée de contrainte sociale diffuse à celle d'une morale nonécrite, dont la force de persuasion serait la manifestation visible, et d'identifier les contenus moraux non-rationalisés de la contrainte diffuse aux normes d'une morale qui se situerait en dehors et au-delà de la société. Certes la distinction ne sera pas toujours facile à faire, en raison même du caractère moins manifeste, plus mystérieux, et nous dirions même plus sacré, pour reprendre le terme de Durkheim, de la contrainte diffuse. Ce mystère conduit parfois l'observateur à y voir la manifestation d'un ordre supérieur, de nature surnaturelle, et à y rattacher une forme de présence de Dieu dans la vie sociale. Nous ne pensons pas devoir entrer dans de tels rapprochements; nous nous contentons d'analyser le phénomène de la contrainte diffuse tel qu'il apparaît à l'observation des sociétés actuelles, en cherchant à y découvrir ce qui la rattache à l'obligation morale et ce qui l'en distingue.

Remarquons tout d'abord que les moeurs, les coutumes, les traditions, les modes et les tabous se présentent de façon souvent beaucoup plus

5 Allusion aux travaux célèbres de Lucien Lévy-Brühl, La mentalité primitive (1922) et $L$ 'âme primitive (1927), ainsi que ceux de Claude Lévi-Stauss, en particulier Les structures élémentaires de la parenté (1949) et La pensée sauvage (1962). 
contraignante que les lois. Il n'est pas nécessaire de brandir la menace du gendarme pour les faire respecter. Pourtant l'absence de gendarme et de sanction officielle et codifiée ne signifie pas que la contrainte diffuse ne connaisse pas de sanction, au contraire. Cette sanction se trouve simplement dans la désapprobation d'autrui, dans l'exclusion morale et psychologique qu'elle implique, dans la culpabilité imputée par le jugement d'autrui, et il semble à première vue que cette désapprobation, souvent non-verbale et dès lors non-justifiée, soit beaucoup plus fortement crainte que la condamnation de la justice.

Dans le cas de l'éthique de la prospérité, par exemple, dont la contrainte spécifique est un exemple type de contrainte non-légale, l'individu peut juger par lui-même, par la simple comparaison de son statut matériel avec celui de son entourage, si son comportement correspond aux attentes de son milieu. Les signes extérieurs de richesse garantissent la reconnaissance sociale et assurent, dans une large mesure, l'intégration souhaitée. L'observation quantitative des revenus, ou la valeur marchande des compétences sur le marché du travail, constituent souvent l'instrument de pression le plus puissant de la contrainte morale qui accompagne la poursuite de la prospérité. Que devrait-on dire également de la honte que doit ressentir le pauvre devant l'arrogant étalage de la richesse que demande une telle éthique?

Doit-on en conclure que la contrainte diffuse renferme de ce fait une part plus grande d'obligation? Est-ce que la désapprobation morale d'autrui, qui sanctionne immanquablement la violation d'une coutume ou d'un tabou est plus forte et plus contraignante que la loi?

Pour rendre compte de l'apparente supériorité de l'influence de la contrainte diffuse, nous devons admettre que la sanction sociale non légale qu'est la désapprobation d'autrui, touche de plus près la conscience morale du sujet que la sanction légale ne peut le faire. Ce n'est donc pas dans le contenu du jugement qu'implique la contrainte morale diffuse qu'il faut chercher ce qui la différencie de la contrainte organisée, mais dans son rapport au sujet. Or ceci ne s'explique que par une disposition du sujet luimême qui le rend plus sensible, ou si l'on veut plus réceptif, aux injonctions de la contrainte diffuse qu'à celles de la contrainte organisée. Cela veut dire que si la contrainte diffuse peut pratiquement forcer le sujet à agir d'une façon plutôt que d'une autre, il doit y avoir en l'homme une disposition, une tendance à s'y soumettre. C'est cela que nous devons tenter d'expliquer.

En rapport avec ce que nous avons dit plus haut de la norme, nous savons que notre action est soumise au jugement de valeur qu'autrui porte sur elle. Il l'approuve ou la rejette au regard des valeurs auxquelles la communauté des sujets-autrui adhère. Or la conscience de savoir que notre action est jugée de cette façon influence le sujet jusque dans son intention, et cela pour deux raisons. D'abord, la désapprobation d'autrui nous rejette hors du groupe, nous coupe du lien qui nous rattache au groupe, et la peur de la solitude créée par cet ostracisme moral nous pousse à jouer le jeu, pour ainsi 
dire, que la société nous demande de jouer. Voilà pourquoi, en dehors même du contenu de la contrainte morale diffuse, nous essayons de correspondre le plus étroitement possible à ce que la société attend de nous. La contrainte n'est pas autre chose, dans ce cas, que la manifestation de notre peur de la solitude. C'est cette peur d'être seul et rejeté, cette angoisse de l'exclusion, qui, selon Bergson, pousse le criminel à se dénoncer.

Mais la contrainte n'est pas seulement la peur d'être rejeté du groupe; elle est beaucoup plus profonde et s'enracine au coeur de la conscience morale elle-même. Quand nous avons parlé de l'obligation de se faire, nous avons montré que l'action morale n'était pas seulement l'actualisation d'une valeur, mais représentait également une création de soi. L'homme se faisait bon ou mauvais par sa propre action. Or, du fait que le jugement de valeur vient d'autrui, que c'est la société qui finalement approuve le Bien et désapprouve le Mal, c'est par elle que je saurai si je suis bon ou mauvais. De cette manière, faire ce que la société demande, même si cela n'est pas formellement légalisé, semble être la voie la plus directe et la plus sûre pour s'assurer de sa propre valeur. La contrainte sociale, dans ce cas, n'est pas celle qui nous pousse à faire ceci ou cela, mais celle dans laquelle nous reconnaissons la forme la plus directe d'un devenir satisfaisant. Cette attitude ne demande même pas à la conscience de référer son action à un Bien ou à un idéal qui se présenterait comme un absolu, mais simplement à se connaître comme bon, ou à être menacé d'être reconnu comme mauvais, à travers la seule approbation ou désapprobation d'autrui. Ainsi, la peur de la solitude et notre désir de nous savoir bons par l'approbation d'autrui sont les fondements de cette disposition subjective qui donne à la contrainte sociale diffuse sa force de persuasion.

Elle se concrétise, selon les théories psychanalytiques, par la création d'une conscience morale supérieure, le sur-moi, dont l'origine est l'image parentale idéalisée, transposée dans la perspective religieuse à un niveau surnaturel dans l'image d'un Dieu qui devient lui-même l'idéal moral. ${ }^{6}$ L'explication psychanalytique confirme notre affirmation de l'auto-création de l'homme par lui-même à travers l'action morale. L'idéal n'est pas simplement une valeur, mais s'incarne dans une personne dans laquelle se retrouvent non seulement des valeurs d'intention mais des valeurs d'être. Le sur-moi de la psychanalyse, c'est le moi que je veux devenir et que je projette dans la réalité avant de le construire par mon action. Existant pour moi, ce deuxième moi s'impose à moi et sa présence rend la contrainte morale possible.

Les sociologues, de leur côté, affirment que ce phénomène du sur-moi est d'origine purement sociale. Par lui, l'homme intériorise les exigences

6 Nous avons vu comment Fulliquet, par exemple, avant les theories de la psychanalyse, a utilisé ce processus psychique pour fonder l'idée d'obligation. Voir plus haut, p. 68. 
de la société, et le degré de cette intériorisation, mesuré à l'aune de la force réelle de la contrainte, manifeste le degré de conscience morale du sujet. La présence effective du groupen'est même plus nécessaire puisque l'intériorisation du sur-moi social remplace la contrainte sociale manifeste. C'est ainsi que le sujet, dans la solitude de sa chambre, ou semblable à Robinson Crusoë sur son île, sans aucun témoin capable de symboliser la société et à mille lieues de toute possibilité d'un jugement social de fait, continue d'agir conformément aux exigences sociales. Le sujet s'illusionne en croyant qu'il agit au nom d'une obligation fondée dans l'absolu, alors qu'il ne fait que poursuivre, par le truchement d'un sur-moi social intériorisé, les fins que la société lui impose.

Dans ces deux théories, psychanalytiqueet sociologique, ce n'est finalement pas le moi réel qui décide et qui agit, ce n'est pas un authentique sujet conscient de lui-même, libre et responsable, mais un agent plus ou moins déterminé par sa crainte de la solitude et conditionné par son désir d'être jugé bon par ses semblables. La contrainte sociale diffuse impose en fait une moralité de pur contenu, simplement matérielle, qui ne tient pas compte de la forme subjective et de la créativité personnelle indispensable à toute action morale.

La théorie sociologique, en effet, n'explique pas l'attitude hypocrite qui consiste à présenter une image de soi conforme à la morale de la société en vue d'obenir l'approbation de ses semblables, mais qui ne maintient pas de cohérence du comportement dès qu'un jugement devient impossible. La moralité construite sur la contrainte sociale ne tient pas compte du besoin de sincérité. Or, si la sincérité est, comme nous l'avons vu, en raison même de la cohérence qui doit exister entre la personne qui agit et le sens de son action, une partie intégrante de l'attitude morale authentique, l'obligation ne peut être assimilée à la contrainte sociale diffuse qui n'en tient pas compte et qui ne demande pas, de la part du sujet, un engagement personnel total. La contrainte ne demande finalement qu'une conformité de l'action individuelle avec les valeurs du groupe, mais elle ne porte aucun jugement sur l'origine de cette conformité, et n'interprète pas la valeur morale de la peur ou de la faiblesse, de la crainte de la solitude ou du manque de maturité personnelle, qui en sont la plupart du temps la cause directe. Il n'y a en aucun cas, dans la contrainte, un appel à l'engagement et au dépassement de soi. C'est pourquoi elle ne peut être authentiquement morale.

La contrainte sociale, organisée ou diffuse, n'est finalement qu'une illusion de l'obligation, car elle repose sur une morale tronquée, une morale qui se passerait du sujet si cela était possible. Certes la contrainte suppose la liberté, comme nous l'avons souligné, mais c'est une liberté qui doit être dominée, et non pas créatrice, une liberté dont la société se méfie et qu'elle essaie de vaincre. La norme qu'elle propose, d'autre part, est le plus souvent construite sans la contribution des sujets qui s'y engagent et qui lui donnent sa pleine substance. Le sujet qui lui obéit est donc beaucoup plus un instrument qu'un agent créateur de moralité. C'est pourquoi les théories qui 
tentent de découvrir l'obligation dans la contrainte sociale sont toujours conduites à supposer un être supérieur qui s'impose aux sujets, une société ontologique et personnelle, comme chez Durkheim, ou une force vitale comme chez Bergson.

\section{Le dépassement de la contrainte morale}

Pourtant la moralité est sociale, et la contrainte existe. On ne peut nier que l'homme est plongé dans une situation morale qui le dispose à subir cette contrainte et à lui obéir. Mais ce n'est pas parce que la morale est sociale que l'on doit postuler une société-substance qui en serait le seul fondement. C'est là que ces théories sont illusoires. C'est moralement que la société s'impose, non pas ontologiquement. C'est l'unique sens de notre postulat. L'existence de la contrainte implique l'existence d'un conflit moral dans lequel, à juste titre, la société et le sujet s'opposent. Ce conflit ne peut être résolu par une victoire à sens unique de l'un sur l'autre, mais, comme nous l'avons souligné, par un dépassement dans lequel aucune des parties du conflit ne renonce à soi-même. La croissance de conscience des sujets implique donc la croissance de cette société-sujet de nature morale que nous postulons. Tout conflit se résoud dans un dépassement créateur. L'obligation réelle se trouve donc au delà de la contrainte.

Si l'obligation morale authentique non seulement suppose la liberté, mais en est la révélation, nous dirons que la morale de la contrainte est une morale de passage, un lieu intermédiaire où le moral et l'amoral se rencontrent et se mêlent, là où l'action n'est pas encore totalement libérée des déterminismes qui la conditionnent et n'est pas encore parvenue au niveau de la moralité totale.

Pour passer au stade supérieur, à celui de la vraie morale dans laquelle l'obligation se manifeste pleinement, il faut que la contrainte soit mise en échec, que la société-objet fasse place à une société transformée. L'explication bergsonienne nous semble représenter, parmi les diverses théories auxquelles nous pouvons faire appel, les éléments clés qui permettent de faire apparaittre la nature du saut qualitatif qui fait progresser l'homme vers une moralité plus ouverte et plus complète, en le faisant passer du monde de la contrainte sociale à celui de l'obligation réelle?

Le changement qui s'opère n'est cependant pas dû à un changement dans la nature de la condition morale, mais à un changement de situation. C'est la situation de guerre, ou de conflit entre deux sociétés, selon Bergson, qui relativise la contrainte morale de la société, ce n'est pas la société elle-même qui aurait changé de nature ou qui aurait abandonné, pour des raisons

7 Voir plus haut, p. 81 et 82. 
inexplicables, la contrainte par laquelle elle assurait la moralité sociale nécessaire à sa survie. La transformation ne se trouve pas dans l'objectivité de la société, mais dans l'intention de l'agent moral. La norme sociale ne peut plus constituer l'objet désirable de son vouloir puisqu'elle est mise en échec par ses propres contradictions. Le sujet se retrouve donc libre, non contraint, disponible pour créer de nouvelles valeurs. C'est l'échec de la contrainte qui constitue réellement l'obligation de créer des valeurs morales, comme nous l'avons vu plus haut. C'est donc à partir des antagonismes entre groupes sociaux, entre diverses sociétés-objets, que peut naître chez les individus une ouverture vers un bien moral supérieur, et la conception d'une société nouvelle transformée par la moralité. Nous verrons aussi que c'est dans la prise de conscience de certaines injustices que l'homme s'ouvre à la possibilité d'un niveau supérieur de justice, et peut ainsi faire progresser l'humanité. L'obligation qui naît ainsi de l'échec de la contrainte et de son dépassement est d'une autre nature, et doit être considérée comme authentiquement morale.

Pour qu'il y ait cependant un véritable dépassement de la contrainte, il ne suffit pas d'affirmer, comme le fait Bergson, que l'intention vise un objet nouveau, l'humanité en général au-delà des sociétés particulières. La réalité de la contrainte ne vient pas seulement du fait que les sociétés particulières sont des sociétés closes, mais également de cette conception de la morale qui fonde le devoir et situe la finalité de la moralité en dehors du sujet moral lui-même. C'est pourquoi, dans la perspective bergsonienne, le saut de nature qui nous fait échapper à la contrainte sociale nous soumet finalement à la contrainte de l'élan vital dans lequel le sujet se perd. Le véritable dépassement auquel le conflit des sociétés peut et doit nous conduire, est celui qui consiste non pas à reporter à un autre niveau la même conception de la morale, mais à renverser cette conception en changeant la finalité même de la moralité. C'est ce que nous appelons le uretour au sujet responsable» dans ce travail.

L'obligation morale demeure donc illusoire si l'on tente de la fonder dans une réalité extérieure, ou même supérieure au sujet moral qu'est l'homme concret et individuel. Le vrai dépassement de la contrainte morale n'est pas celui qui consiste à placer la conscience à des niveaux supérieurs d'universalité abstraite, mais au contraire celui qui l'aide à trouver l'absolu moral lui-même dans l'individualité des sujets, toi, moi, nous. Ce dépassement, qui est aussi un passage du moi au nous, se fait par le partage de la responsabilité. L'acceptation de ma moralité sociale procède d'une reconnaissance, dans les valeurs imposées par la contrainte sociale, d'une responsabilité du «nous» vis-à-vis du «moi». C'est le passage de la conscience d'une obligation individuelle à celle d'une responsabilité commune, en d'autres termes, d'un jugement réciproque d'imputabilité. Ainsi, transformer la norme vécue en une loi universelle valable pour tous et dans toutes les situations, serait la désincarner, l'abstraire, la séparer de sa source vivante et de sa finalté qu'est l'homme individuel, existentiellement responsable de 
ses actes dans le cadre d'une société concrète dont il partage la responsabilité sociale. Dépasser la contrainte signifie donc que la moralité n'a pas d'autre fin que l'homme lui-même, membre d'une société-sujet, et que c'est dans leur responsabilité commune seulement que peut s'incarner l'obligation.

\section{Obligation formelle et obligation matérielle}

L'affirmation du sujet, comme mise en question de la contrainte sociale, est sans cesse formulée dans ces pages au nom de la dimension formelle de la morale. La répétition de ces arguments semble suggérer que l'obligation est formelle, et uniquement formelle; c'est à ce titre en effet que nous avons refusé de l'identifier avec la contrainte d'une société-objet. Ceci semble d'autant plus évident que cette contrainte, - qu'elle soit manifeste dans le manque qu'est la contrainte intérieure, ou qu'elle soit diffuse, à la limite irrationnelle, quand elle est sociale - a toujours été rattachée à quelque contenu moral, à une matière morale. C'est en effet par une matière morale bien précise que la société se maintient en existence. C'est par elle que la société se définit, et la moralité qu'elle propose est toujours une moralité fondée sur son contenu. Faut-il donc admettre que, si la contrainte fondée sur cette matière morale n'est pas l'obligation, celle-ci ne peut être que formelle?

Répondre positivement à cette question serait affirmer que l'obligation ne se rattache qu'à la forme de la moralité, la subjectivité, et n'a rien à voir avec l'autre dimension, c'est-à-dire le bien et le mal, le moral et l'immoral. Suffit-il d'agir par devoir et ne pas se demander ce que ce devoir exige? La moralitén'est-elle que la manifestation d'une obligation, et non pas simplement l'actualisation d'une norme? Est-il vraiment possible de dire d'un homme qu'il est juste sans tenir compte de ce qu'il fait?

Il est évident que si la moralité n'était que formelle, l'obligation forcerait l'homme à construire la moralité à l'intérieur d'une subjectivité dont il ne pourrait s'échapper.L'homme deviendrait prisonnier de lui même, l'intention niée car sans objet, la moralité identique à un être non transformable. Il n'y aurait pas de création possible. Une morale purement formelle et purement impérative nierait le devenir humain. Exclusivement subjective, à la limite solipsiste, la moralité serait la négation du sujet lui-même. Comment saurais-je que je suis moi si l'autre n'existe pas? Comment puis-je affirmer mon action comme mienne si je ne la situe pas par rapport à d'autres?

D'autre part, si la morale ne se souciait que des biens et des buts, visant l'actualisation d'une moralité en soi, indépendante des sujets, l'homme ne deviendrait qu'un instrument de la vie sociale, il devrait renoncer à sa liberté, et seule la contrainte recevrait le nom de devoir.

Dans les deux cas, celui d'une morale exclusivement formelle et impérative, ou celui d'une morale matérielle des seuls biens et de la finalité, la moralité 
se situe au-dessus, ou en dehors, de l'homme et fait de celui-ci l'instrument de celle-là. Par contre si nous croyons que l'homme peut se dépasser, et que nous faisons de la moralité un intrument de la construction de l'homme, l'obligation ne peut être ni purement formelle ni purement matérielle. Elle doit nous obliger à nous vouloir nous-mêmes et à vouloir le Bien, c'est-àdire à placer l'homme au-delà et au-dessus de la moralité.

C'est au niveau de l'existence vécue, et non pas seulement dans l'ordre conceptuel, que nous pouvons saisir cette double dimension de l'obligation et en comprendre tout le sens, à la fois formel et matériel, dans l'accomplissement des potentialités humaines dans une personne. L'action totalement morale à laquelle nous appelle l'obligation, est donc l'action qui a pour but l'épanouissement du sujet et de celui d'autrui dans une relation de personnes.

La notion de personne exprime ce double fondement de la morale et de l'obligation qui s'accomplit dans l'idée d'une société à construire. La morale n'est donc fondée ni sur l'idée d'un Bien moral qui ne serait actualisé que par la contrainte, ni sur l'idée d'un devoir purement formel qui emprisonnerait le sujet en lui-même et le priverait d'épanouissement créatif. La morale s'appuie sur ses deux dimensions.

Finalement, il n'existe pas non plus de moralité-chose, un être en soi indépendant de l'homme, car c'est toujours l'être humain lui-même qui demeure le but ultime de la moralité qu'il construit. C'est pourquoi nous dirons, en conclusion de cette réflexion, que l'obligation morale n'est ni formelle ni exclusivement matérielle, car elle n'a de sens que comme appel des sujets les uns envers les autres, qui s'invitent mutuellement à s'épanouir en devenant des personnes. Le but de la moralité, en effet, n'est ni sa matière ni sa forme, ni le Bien ni le Devoir, ni l'objet ni le sujet, mais les personnes. C'est par rapport à elles, finalement, que l'obligation morale trouve sa signification. 


\title{
Chapitre 6
}

\section{L'obligation envers autrui, ou la société-sujet}

\begin{abstract}
C'est lorsque le monde perd son sens que peut commencer le réenchantement du sujet.
\end{abstract}

Alain Touraine Critique de la modernité

L'obligation envers la conscience conduit à reconnaître, dans le devenir de la personne, l'identification progressive de la conscience de soi et de la conscience morale, de la conscience de l'être et de celle du Bien, dans une unité de l'être-de-la-personne qui se manifeste essentiellement comme être moral. 'Il y a donc une présence de la valeur dans le devenir concret de l'être humain, valeur fondée sur l'expérience de l'extériorité et de l'objectivité de la présence d'autrui, considéré conjointement comme autrui collectif et individuel. Nous venons justement de voir que la présence d'autrui se manifeste comme société, société-autrui, prise comme réalité symbolique des autres, appréhendés collectivement. L'obligation envers soi-même conduit donc à la découverte d'une obligation envers la société. Nous dépassons ainsi le pur formalisme de la subjectivité, l'individualisme de toute forme d'eudémonisme et pouvons donner à la moralité un fondement rattaché à la création des valeurs.

\footnotetext{
${ }^{1}$ Voir fin du Chapitre 4.
} 
Pourtant ce n'est pas simplement dans le seul fait d'exister qu'autrui intervient dans la conscience morale du moi; c'est aussi et surtout parce qu'il est un être moral et que sa moralité m'importe. L'expérience directe de mon rapport avec autrui est donc une expérience de responsabilité commune, volontairement partagée. C'est le cas avec chaque individu pris séparément; mais c'est aussi le cas dans mon rapport à la société, postulée comme société-sujet.

L'existence d'autrui est à l'origine de la valeur, et particulièrement de la valeur idéale qu'est la norme, à l'aune de laquelle mon action est jugée morale ou immorale. Penser aux devoirs envers autrui, ou envers la société m'introduit donc directement dans la dimension matérielle de la morale et mon obligation envers autrui s'enracine dans la conscience des valeurs que nous partageons. Nous devrons donc analyser notre obligation envers autrui à partir de la norme, ou valeur idéale, car c'est à partir de cette norme, telle qu'elle m'est proposée dans ma rencontre avec autrui que j'en fais l'expérience directe. Du point de vue de la valeur, ma première obligation est donc une obligation envers le Bien, ressentie comme responsabilité partagée.

Il devient donc nécessaire de définir clairement la relation entre valeur et obligation. L'obligation se fonde-t-elle sur la matérialité de la valeur, comme le suggère par exemple Max Scheler, ou au contraire faut-il, à la suite du formalisme kantien, déduire qu'une conduite n'est valable, ou digne de la valeur, que parce qu'elle est fondée sur le devoir? La réponse à cette question dépend essentiellement de la conception de la valeur que nous retiendrons.

Dans la mesure où, à la suite de Scheler, on ne reconnaît comme valeur que les «valeurs-de-la-personne», c'est dans l'être même des personnes que l'obligation trouve son fondement ultime. Mais il nous faut aussitôt admettre que ce fondement n'est pas absolu, qu'il n'est ni proposé comme une évidence, ni déterminé dans son être, puisque la personne, comme nous l'avons souligné à maintes reprises, ne peut être conçue que comme un devenir. En conséquence, la valeur qui s'y rattache est toujours une valeur en devenir, une valeur en perpétuelle transformation. Il n'est donc pas possible d'imaginer l'obligation en dehors de la situation dans laquelle la construction des personnes elles-mêmes prend place, par interaction individuelle et collective, et qui n'est pas autre chose que la confirmation d'une société fondée sur les valeurs, ce que nous appelons la «société-valeur», ou sociétésujet, distincte du social empirique que nous avons appelé la société-objet.

4 Dans la perspective de cette société-sujet que nous postulons du point de vue de la philosophie morale, autrui et société deviennent synonymes. Parler d'autrui ou de la société devient donc indifférent. Mais iln'en est pas de même dans une société objet, au sein de laquelle les individus ne sont pas nécessairement «autrui» l'un par rapport à l'autre, mais complémentaires dans leur soumission aux déterminismes sociaux. 
Mais des questions préalables doivent avoir reçu des réponses avant d'aller plus loin. Mon obligation envers autrui serait-elle déterminée par une situation précise, et non pas par la personne de l'autre qui en est la finalité concrète? Est-ce qu'il n'y a pas certaines confusions sur le sens de cette société-sujet, et ne reconstruisons-nous pas une nouvelle réalité objective qui se retrouverait, comme un obstacle, entre moi et la personne de l'autre? Répondre par l'affirmative impliquerait que nous devrions en revenir à une approche formelle de l'obligation, où la forme serait la situation elle-même, et abandonner l'idée de trouver dans un contenu-de-valeur la source d'une quelconque obligation envers autrui. L'amour, en tant que situation particulière entre deux personnes, serait-il alors la forme la plus pure de cette obligation? La société le seul absolu qui mettrait toutes les personnes en présence l'une de l'autre?

Ces différentes questions permettent de complèter le tableau des multiples manifestations de l'obligation par lesquelles nous tentons d'en préciser la notion. Car l'obligation est toujours concrète, et les théories qui tentent d'en rendre compte sont toujours mises en échec, en tant que théories, par la réalité de l'engagement moral des personnes. Comprendre le sens de l'existence demeure une tentative enthousiasmante et digne de tous les efforts, mais cela ne remplace pas le fait que c'est toujours dans la vie concrète, hic et nunc, que l'homme doit prendre des décisions irrévocables.

\section{Le contenu de l'obligation et les vertus}

Dans la mesure où l'obligation envers autrui ne se révèle pas dans la dimension formelle de la morale, à partir de laquelle l'obligation envers soimême s'est progressivement dévoilée, la première question que nous devons nous poser à son sujet est de savoir quel contenu cette obligation renferme et comment nous pouvons le connaitre. En fonction de ce que nous avons affirmé jusqu'à maintenant, celui-ci ne peut être qu'un contenu-de-valeur, c'est-à-dire un contenu essentiellement lié à ce devenir de la personne qui est apparu comme fondement de la condition morale de l'homme. C'est donc à partir de l'expérience des valeurs morales que nous devons tenter de révéler la signification de l'obligation envers autrui.

Reconnaissons tout de suite qu'iln'est pas possible, dans ce but, de partir d'un simple inventaire factuel des valeurs, normes et maximes morales communément admises, pour remonter, à partir d'elles, à l'obligation qui les soutient. La raison en est simplement que leur énoncé ne permet pas, à première vue, de distinguer celles qui se rattachent à une authentique obligation morale de celles qui ne sont que l'objet de la contrainte sociale. Ce n'est qu'à partir de la norme elle-même, considérée comme valeuridéale, qu'il est possible de distinguer les valeurs qui ont un véritable contenu d'obligation de celles qui ne sont qu'une illusion suscitée par 
l'objectivité sociale. Comme nous le répétons sans cesse, il n'est pas possible de limiter notre approche de l'obligation à son simple contenu matériel, mais devons sans cesse la rattacher à la double dimension de la moralité.

D'autre part, nous ne croyons pas non plus qu'il soit possible de trouver le contenu de l'obligation dans ce qu'on appelle les vertus. Disons très brièvement, sans entrer dans une discussion approfondie de la notion en elle-même, que la vertu, prise comme une dispositon particulière ou une inclination spéciale de la volonté, n'est pas nécessairement rattachée à un contenu-de-valeur. En effet, ce n'est pas par le truchement du Bien que nous reconnaissons la vertu, mais par le biais de ce qui est moral. Est vertueuse l'inclination qui soutient la volonté à agir moralement. Or, comme nous savons que le terme moral revêt deux significations, formelle et matérielle, nous devons admettre qu'il y a des vertus formelles et des vertus matérielles, ou, si l'on veut, des vertus «envers soi-même» et des vertus «envers autrui», des vertus personnelles et des vertus sociales.

La charité, par exemple, ou mieux dit l'attitude charitable, est évidemment une vertu qui concerne autrui, celle qui veut faire le bien, pour reprendre une expression courante. L'anti-charité serait donc immorale! Mais la vertu de courage, elle, ne concerne que l'attitude du sujet; elle ne porte aucunement sur un contenu-de-valeur, elle est donc formellement morale. C'est une vertu qui exige authenticité et responsabilité. Avoir du courage, c'est admettre que nous ne pouvons connaître à l'avance le résultat de nos actions, mais que nous nous y engageons cependant totalement. Il faut du courage, en effet, pour être authentiquement moral, c'est-à-dire pour assumer l'entière responsabilité de nos actes, indépendamment, et parfois même en dépit, du contenu de l'action. Or il y a un conformisme moral qui se fonde avant tout, et presque uniquement, sur l'approbation d'autrui, donc sur un contenu moral, qui ne demande aucun courage et fait de la vertu une simple habitude de conformité. La vertu, dans ce cas, ne demande pas d'invention. Mais le saint et le héros, par contre, sont pleinement courageux car, en orientant leur action vers une moralité supérieure, à travers un dépassement de la "sociétéobjet», ils acceptent d'affronter le conformisme et prennent le risque d'une moralité solitaire. Il faut en effet du courage pour construire un être qui n'est pas encore, la personne en devenir. Il y a, d'autre part, des vertus qui sont choquantes de conformisme éthique et qui peuventêtre parfaitement amorales. C'est pourquoi la vertu qui résulte de la seule contrainte sociale est très souvent d'une moralité très ambiguë.

Notre attachement à la double dimension de la morale nous empêche de souscrire, sans critique, aux doctrines qui font de la vertu une habitude, une disposition acquise, quasi-définitive, de la volonté,l'habitus de la philosophie scolastique. Pour penser la vertu en ces termes, il faut postuler une finalité morale donnée d'avance, établie dans l'être, à la réalisation de laquelle I'homme doit se conformer. La vertu représente l'aboutissement d'un exercice répété par lequel l'homme devenu vertueux est arrivé à vouloir - 
nous devrions dire, est conditionné à vouloir - par une spontanéité acquise, le contenu du devoir. Max Scheler le dit en ces termes: «La vertu est le pouvoir, immédiatement vécu, de faire quelque chose qu'on doit faire». ${ }^{3}$

Mais il estévident que ces conceptions conduisent, presque automatiquement - ce qui n'est pas une expression exagérée puisque l'automatisme est souhaité - à transformer la vertu, et par elle la morale, à l'accomplissement d'un légalisme extérieur, de nature exclusivement sociale, et à tuer l'obligation intérieurement vécue. L'obligation aurait-elle encore un sens pour un homme dit vertueux qui désire de toute façon faire, en raison de ses penchants acquis et de la disposition de sa volonté, ce que cette obligation lui propose de faire? L'impasse dans laquelle tombent ces doctrines de la vertu vient de ce qu'elles considèrent l'obligation uniquement dans la dimension de son contenu, ou de sa finalité matérielle, et celle-ci étant achevée par un acte bon, la fonction de l'obligation étant accomplie dans l'acte, c'est la vertu qui se substitue à l'obligation pour assurer la morale. La conscience de l'obligation n'aurait donc pas d'autre but que de conduire à la vertu, et la moralité ne commencerait qu'avec celle-ci. La vertu deviendrait, de ce fait, le contenu de l'obligation, un contenu par rapport auquel l'obligation ne serait que fonctionnelle. Mais cela ne nous éloignerait pas beaucoup de l'effet de la contrainte sociale.

Dans un autre sens, nous constatons que la vertu de courage peut conduire elle-même à la découverte de l'obligation. C'est dans ce cas la vertu qui serait fonctionnelle. C'est pourquoi il ne faut pas considérer la vertu dans son seul rapport au contenu moral qu'elle est supposée mettre en oeuvre, mais également par rapport à la forme de la morale qui implique justement la vertu pour se définir. L'obligation exige le courage, elle n'y conduit pas. C'est donc en distinguant les vertus formelles, comme le courage ou la sincérité, des vertus matérielles, comme la charité ou l' honnêteté, qu'on évite d'assimiler la vertu en général à un contenu de l'obligation. Ainsi l'homme vertueux n'est pas celui qui semble avoir moins besoin de l'obligation pour être moral, mais plutôt celui qui est capable d'en connaître plus authentiquement les exigences. Le saint n'est pas, avant tout, celui qui fait le bien spontanément mais celui qui, ayant une conscience plus aiguë de sa propre imperfection, entend plus fortement l'appel de l'obligation pour vaincre la faiblesse dans laquelle il se reconnaît et ainsi progresser dans la charité et la sagesse.

\section{Les morales didactiques}

Or nous devons savoir que ce besoin de rechercher à tout prix le contenu de l'obligation s'enracine dans l'intention de la majorité des morales, qui

${ }^{3}$ Max Scheler, Le formalisme en éthique, p. 221 
ne visent pas la contemplation des vérités éternelles, mais abordent les vérités morales pour guider la conduite humaine. Certes c'est la tentation de la morale de pouvoir enseigner à l'homme ce qu'il doit faire et ce qu'il doit choisir. Le but est alors de situer l'action dans le domaine de la certitude. Simone de Beauvoir a exprimé cette tendance quand elle écrit que le propre de toute morale «est de considérer la vie humaine comme une partie que l'on peut gagner ou perdre, et d'enseigner à l'homme le moyen de la gagner». ${ }^{4}$ Il y a en effet une intention didactique en toute éthique.

Mais si nous nous plaçons dans la perspective de l'anthropologie morale que nous esquissons dans ces pages, nous comprenons que la partie qui est à gagner, ou à perdre, est le propre être de l'homme, et qu'il peut la gagner ou la perdre selon qu'il est capable ou non d'inventer les valeurs de sa propre personnalisation.

La question essentielle est donc de savoir comment il est possible d'enseigner à l'homme l'invention, comment lui montrer de l'extérieur ce qu'il ne peut découvrir qu'en lui-même et par lui-même? A la limite, c'est là que se situe la difficulté centrale. C'est pour éviter ces difficultés, semblet-il, que beaucoup de morales ont délibérément choisi de se limiter à certaines orientations éthiques en raison de la facilité avec laquelle elles parvenaient à des conclusions didactiquement valables. La connaissance d'un contenu définissable de l'obligation fait partie, croyons-nous, de ces illusions dans lesquelles les morales didactiques croient trouver leur certitude. Il est utile, c'est du moins notre conviction, de démystifier ces fausses espérances pour dévoiler, autant que possible, la nature de l'obligation envers autrui.

\section{Les morales de la prudence}

Certes la connaissance des choses n'est pas inutile, car c'est à partir de l'appréhension de l'existence donnée que la morale commence. Mais elle n'est qu'un point de départ, et non un aboutissement. Nous l'avons déjà répété, la finalité de la moralité ne se trouve pas dans le monde achevé. Toutes les morales qui ont tenté de trouver dans une connaissance objective de la vie et du monde la source des valeurs et des normes de l'action sont restées figées et statiques. Ce sont des morales de la prudence qui restreignent la personne.

Or c'est dans les morales de l'invention et du risque que la personne se conquiert. Certes, il n'y a pas de sagesse morale sans prudence, mais cette prudence ne consiste pas à se voiler les yeux; elle doit au contraire se

4 Simone de Beauvoir, Pour une morale de l'ambigüté, p. 32-33. C'est nous qui soulignons 
justifier par rapport au but à atteindre, et ne pas se contenter d'assurer ses arrières. La prudence ne consiste pas à s'arrêter devant un obstacle, mais bien à le saisir pour pouvoir le surmonter. Or de telles victoires sont à la fois des victoires sur le monde et sur soi-même. On ne progresse pas en respectant les limites que les obstacles nous imposent.

Il y a, par exemple, des morales d'inspiration biologique qui pourraient être identifiées à ces morales que nous devons qualifier de prudence passive. Certes l'homme ne peut aller au-delà de sa condition physique, mais si la liberté se limite à choisir les déterminismes qui sont utiles et à rejeter ceux qui apparaissent nuisibles ${ }^{5}$, on crée ainsi un eudémonisme empirique dont le résultat se présente comme un équilibre parfait entre les désirs humains et leur utilité, entre le plaisir et sa conformité à l'existence telle qu'elle est donnée. En s'appuyant sur la connaissance objective des choses, et en faisant appel à la prudence et à l'intelligence, ces morales tentent de convaincre l'homme d'agir selon les normes qu'elles proposent. La présence d'un paradigme puissant contribue énormément au succès de telles morales. Mais c'est sur la conviction, non sur l'obligation, que ces morales fondent la moralité. C'est négativement, par le rejet de l'inutile, qu'elles espèrent conduire au Bien. Finalement, seul le Mal pourrait faire l'objet d'un libre choix, à la façon de GOETZ, puisque le Bien est donné dans les choses et découvert par la connaissance que nous pouvons en avoir. Il n'y a donc pas de devenir possible ni d'invention.

Ces morales pourraientêtre considérées comme des versions contemporaines et scientifiques de la morale d'Epicure. Il est vrai, en effet, que ce serait gaspiller l'homme, son corps et la nature dans laquelle il vit que de les dépenser - sans prudence, il faut l'admettre - dans des activités inutiles et dangereuses. Les réactions actuelles de la conscience écologique contre les excès du développement industriel sont de cette nature et sont empreintes, dans leur principe du moins, de grande profondeur morale. Epicure le disait déjà à sa manière. Pourquoi manger plus qu'il n'est sain et raisonnable, si les désagréments entraînés par ces excès sont plus graves que le plaisir qu'on peut en obtenir? Pourquoi se perdre dans le libertinage, si le plaisir obtenu doit être payé par la maladie du corps et le blâme d'autrui? Pourquoi, dirions-nous aujourd'hui, multiplier nos centrales énergétiques à grand risque si le confort obtenu peut entraîner la destruction de nos espaces et menacer nos vies? Face à ces questions, la sagesse morale doit s'identifier au respect de l'ordre des choses données, et l'être moral s'accomplit donc dans la modération.

Kant définit la prudence comme une compétence moralement neutre qui donne lieu à des impératifs pragmatiques et a pour but le bonheur personnel ou social, à la différence de l'impératif éthique, ou catégorique. On peut considérer dans la même perspective l'utilitarisme d'un Stuart Mill, par

5 Voir par exemple, Paul Chauchard, Biologie et morale, p. 134 
exemple, et il est important d'y faire allusion par rapport à l'éthique de la prospérité. C'est une morale sociale, fondée sur l'expérience concrète des individus, socialement égocentrique, dépourvue de toute métaphysique, qui propose des vérités utiles de comportement, et qui tente d'entraîner l'adhésion de la volonté en lui montrant en quoi elle aurait tort de ne pas suivre les injonctions que cette morale propose. La tyrannie et le désordre, dans la société, entraînent la révolte et l'anarchie; il est donc préférable de respecter l'ordre et, dans toute la mesure du possible, d'appliquer les moyens nécessaires pour le maintenir. Si tu fais du bien à ton voisin, il te le rendra. Cette morale enseigne donc qu'il est préférable, avantageux, donc bon, d'avoir avec autrui des relations de bon voisinage. L'utilitarisme conduit ainsi à des morales de la majorité, où l'opinion du plus grand nombre remplit peu à peu la fonction de norme et de vérité. Le Bien correspond à ce qui est le plus commun et le plus répandu. C'est donc une morale de convention tacite qui aboutit au conformisme et qui, finalement, conduit à la contrainte, si ce n'est au despotisme, du plus grand nombre. Les morales de la prudence tendent à faire diminuer progressivement, au nom de la sagesse, la valeur de la responsabilité, y compris de la responsabilité collective.

Nous retrouvons, dans le paradigme de l'éthique de la prospérité, une tendance similaire qui conduit, par souci généralisé de l'efficacité et par l'utilitarisme des valeurs de la croissance de la richesse, à une diminution de la responsabilité personnelle devant l'opinion commune. Or, contre une telle «norme de convention» qui n'est qu'une apparence de la véritable valeur morale, Confucius écrivait déjà: "Qu'un homme soit aimé de tous ses concitoyens, cela ne prouve rien. Qu'il soit détesté de tous, cela ne prouve rien. Il faut qu'il soit aimé des bons et haï des méchants. ${ }^{6}$ Le conformisme de l'utilité sociale ne peut donc fonder une authentique obligation envers autrui puisque ses valeurs peuvent, à la limite, se passer de la responsabilité du sujet, et de ce fait nient la personne.

Certes, toutes ces morales sont capables de fournir des décalogues susceptibles d'être enseignés; elles possèdent un contenu communicable. C'est d'ailleurs leur but: convaincre, persuader et, par l'évidence de leurs propositions, entraîner l'homme à agir selon les normes qu'elles proposent. Or il n'y a pas d'argument plus convaincant que de souligner la préférence d'un choix quelconque par l'approbation préalable de tous. Toutefois, cette contrainte par le savoir et la prudence, qui nous semble cependant une illusion de l'obligation, n'appelle pas la personne au dépassement de soi, mais conduit l'homme à s'établir dans l'existence moyenne du groupe. C'est pourquoi nous dirons, à la limite, que les morales du connaître, du savoir, de la vertu, de l'habitus et de la prudence sont des morales de la passivité, en ce sens qu'elles proposent une soumission au réel et non une création de la valeur. Or il n'y a pas de responsabilité dans la passivité, donc pas de faute

6 Pensées morales de Confucius, traduit du chinois par René Brémond, p. 118-119 
possible, pas de risque, ni non plus de possibilité de création du sujet social. A la limite, elles détournent l'individu de son obligation de personne envers autrui et la société.

\section{Le contenu cache l'obligation}

Voilà pourquoi, en cherchant à révéler l'obligation par son contenu et par lui seul, ces morales manquent l'obligation elle-même. La moralité qui s'en dégage est un eudémonisme du juste milieu visant à établir la vie humaine dans l'équilibre et la tranquilité où le Bien se confond avec l'agréable, le vrai avec l'existence, la vie avec le repos. Jean Brun écrit dans son étude sur l'épicurisme: «Le "ne pas varier" fait partie de la sagesse épicurienne, ce statisme par le savoir implique une référence à une félicité suprême, celle des dieux qui demeurent».?

Mais ce statisme peut être un danger pour le devenir humain et le progrès des sociétés. Se prenant illusoirement pour un dieu satisfait de son sort dans l'existence donnée, un peu à l'image de ces bouddhas grassouillets affalés dans leurs rondeurs qui contemplent béatement leur nombril, le sage des morales passives refuse toute tentation de s'ouvrir à un devenir possible. La sagesse du plus grand nombre lui tient lieu de vérité et la satisfaction de l'acquis de suprême vertu. Comment pourrait-il comprendre que sa vraie participation à la divinité se trouve dans un devenir créateur qui, en mettant justement en question ce qui semble acquis, lui donne la possibilité de se grandir?

Notre situation contemporaine est en partie assimilable à ce type de morales, avec un danger plus grand encore, qui vient du fait d'avoir remplacé le devenir humain par le devenir des choses, et de confondre la conquête de soi avec l'accroissement des richesses. Les valeurs qui donnent aujourd'hui un sens aux comportements humains ne sont pas nécessairement des valeurs humaines, mais simplement la valeur matérielle des choses. Il n'est donc pas possible d'y trouver un fondement de l'obligation envers autrui, car ce n'est pas ce dernier, mais la richesse, qui est la finalité ultime de l'action.

Ainsi, dans la mesure où l'on tente de fonder la morale sur le seul contenu de l'obligation, où l'on croit que la sagesse et la vertu peuvent être enseignées, qu'il y a un comportement conforme à l'utilité individuelle et sociale qui peut être inculqué en l'homme par la pression du groupe et de la conviction, on met en place des morales passives recommandant pour l'action les voies les moins dangereuses et les moins troublantes. La tranquillité dans l'acceptation de l'opinion la plus répandue devient donc

7 Jean Brun, L'épicurisme, p. 120 
la sagesse ultime. On fait de la vertu la recherche d'une efficacité bien calculée, un juste milieu entre le trop et le trop peu d'humain, un équilibre entre les forces qu'elle exige de l'agent et les résultats quantifiables que l'action peut espérer. Une telle morale enseigne une prudence qui tue le dynamisme de l'être.

On pourrait, pour y échapper, revenir judicieusement à Aristote, pour qui la phronésis, habituellement traduit par prudentia, mais qui est une vertu intellectuelle plus globale, comprenant non seulement la sagesse que donne l'expérience, mais un sens pratique doté de jugement et d'aptitude à l'action, ne tend pas à la limitation de l'être, mais à sa perfection. "La prudence (phronésis) a pour objet ce qui est propre à l'homme et ce sur quoi peut s'exercer la délibération. Ce qui caractérise surtout l'homme prudent, c'est la délibération bien conduite. ... L'homme de bon conseil, absolument parlant, est donc celui qui tend, en suivant les calculs de la raison, vers ce que l'homme peut réaliser de meilleur».,

Si la morale peut enseigner quelque chose, ce n'est pas la moralité, c'est l'homme. Or celui-ci n'est pas le contenu de la moralité, il en est avant tout la cause et la forme. C'est pourquoi nous disons que la philosophie pratique ne conduit pas à une connaissance du Bien, à partir de laquelle on déduit le contenu de l'action, mais à une connaissance de l'homme comme créateur du Bien. Elle doit être une anthropologie morale. Car le Bien, finalement, n'est pas le résultat d'une connaissance, mais d'une décision. Il n'est pas un objet, mais une valeur, car seule la valeur est capable de donner au sujet, sans le nier, le dynamisme indispensable à la conquête de soi.

La signification de l'obligation envers autrui ne se trouve donc pas dans un contenu de l'obligation qui se poserait comme préalable à l'obligation elle-même. Le jugement de valeur ne précède pas l'obligation, il en surgit. C'est pourquoi la société qui résulte de l'obligation envers autrui n'est pas une société-objet, celle que décrivent les sociologues, les ethnologues et les historiens des moeurs, mais cette société-sujet, celle que construisent les sujets dans l'appel de valeur qu'ils se lancent comme personnes. C'est pourquoi il ne suffit pas d'enseigner la vertu pour permettre à l'homme de gagner son être, il faut lui montrer comment la valeur elle-même se communique par l'obligation.

\section{L'obligation à la valeur}

La question qui se pose ici est de savoir si le Bien existe parce que nous sommes obligés, comme une conséquence de l'obligation, ou au contraire si nous sommes obligés parce qu'il y a un Bien qui préexiste à l'obligation.

8 Ethique de Nicomaque, Livre VI, Chap. 7,6 
Devons nous suivre Hegel, par exemple, qui dit que «le bon est le contenu des devoirs", ${ }^{9}$ ou à l'inverse, affirmer à la suite de Scheler que «toute obligation se fonde sur des valeurs, mais les valeurs ne se fondent jamais sur une obligation idéale». ${ }^{10}$ En d' autres termes, nous devons nous demander s'il y a réellement une obligation «à la valeur», c'est-à-dire fondée sur la valeur, ou si la valeur n'est pas autre chose que la réalité de l'obligation.

La réponse à cette question, dans la perspective de la société-sujet, devrait ouvrir le débat sur les rapports de la société et des personnes en vue de substituer à l'éthique de la prospérité une morale plus proche de la justice.

Il semble, à première vue, que le choix de l'une ou de l'autre position dépend de présuppositions ressortissant davantage au domaine de la théorie de la connaissance, et particulièrement de la connaissance des valeurs, qu'à celui de l'analyse morale proprement dite. Dès lors, il devient apparemment impossible de résoudre le problème de la contradiction de ces vues par une analyse exclusivement morale. Toutefois, nous croyons qu'en rappelant la distinction que nous avons faite entre la dimension formelle et la dimension matérielle de la morale, nous pouvons aborder ce problème dans une perspective où les contradictions qu'il semble impliquer peuvent être dépassées sans que les exigences logiques qui les soutiennent soient méconnues ou supprimées.

D'abord, nous savons maintenant que, dans la mesure où la morale est entièrement assimilée à sa dimension formelle, il n'est pas possible d'affirmer l'existence d'une valeur morale en dehors de cette forme même. Pour Kant, dès lors, il n'y a pas d'autre origine de la valeur morale que le devoir luimême. L'existence d'un Bien, ou sa non-existence, n'importe à la morale que dans la mesure où celle-ci est capable, formellement, de l'affirmer ellemême. En dernier ressort, ce qui est bon est le contenu de l'action que je fais par devoir. Au delà de cette évidence formelle, il n'y a pas de certitude morale possible, et toute affirmation sur le Bien ne peut se présenter que comme un postulat. En conséquence, la seule affirmation qu'il soit possible de faire au sujet du bien, est qu'il constitue ce que le devoir demande. La valeur est donc fondée sur le devoir. Il n'y a pas d'obligation à la valeur, mais simplement une obligation au devoir, le bien moral étant donné, pour ainsi dire, par surcroît.

Le bien dont nous parlons ici est le bien de l'action, c'est-à-dire une valeur qui peut faire l'objet d'un jugement moral de valeur, tel que nous l'avons décrit plus haut. Or Kant ne pense pas pouvoir fonder la morale sur ce bien. Il y a certes un bien, chez Kant, donc une valeur, mais elle est préalable à l'action et se manifeste dans la volonté bonne. Cependant, ce bien de la volonté, cette valeur morale fondamentale reconnue par Kant, est une valeur «dans» l'intention, et non pas une valeur «de» l'intention, c'est-

9 Hegel, Propédeutique philosophique, p. 216

${ }^{10}$ Max Scheler, op. cit., p. 222 
à-dire une valeur qui serait extérieure à l'intention. Il faut donc passer par la forme du devoir pour acquérir quelque certitude sur l'existence de ce bien «dans» l'intention, le devoir ayant la fonction de certifier la bonté de la volonté. Ce qui compte, pour Kant, n'est pas tellement ce que l'homme fait, c'est-à-dire le contenu-de-valeur de l'action, mais avec quelle intention il le fait, c'est-à-dire la "valeur-forme» de l'action. Il y a donc bien une valeur liée au devoir kantien, mais cette valeur n'est pas un contenu de l'action, selon notre conception, mais la forme de l'intention de l'agent. Le devoir assure donc la rectitude morale formelle, la seule valeur morale qui soit considérée chez Kant comme fondement de la morale. C'est parce que le devoir existe que l'action concrète qui en résulte peut être considérée comme bonne. C'est pourquoi nous disons que, pour Kant, le bien moral, le bien de l'action, dépend du devoir.

Mais il n'est alors pas possible de parler dans ce cas d'une obligation envers autrui, qui s' incarnerait dans un contenu-de-valeur concernant autrui. Car ce n'est pas par rapport à autrui qu'il y a une valeur morale, mais uniquement par rapport à la forme même de la moralité. Le bien, en dernier ressort, est pour moi comme pour autrui ce qui est accompli par devoir. Le refus du devoir serait donc à la fois amoral et immoral, et il n'est plus possible de distinguer, dans une telle conception, le jugement moral de fait du jugement moral de valeur. Le fait de la morale, le devoir, en est également la valeur.

La perspective inverse considère le bien ontologiquement, comme une réalité et un contenu-de-valeur qui préexiste à l'intention qui le vise. Le rapport de ce bien à l'intention n'est alors pas formel, mais pleinement matériel. La valeur de la moralité ne se trouve donc plus «dans» l'intention, mais en dehors d'elle, dans la finalité qu'elle vise. L'intention n'est plus alors bonne ou mauvaise en elle-même, elle est seulement bien ou mal orientée. Elle est en elle-même neutre, comme nous avons dit plus haut que la liberté pouvait, en soi, être neutre par rapport à la valeur. Il faut donc chercher dans la réalité du bien la valeur de cette morale qui se referme alors sur sa dimension matérielle. La valeur de l'action vient donc de la conformité de l'intention avec une «valeur-finalité» donnée à l'avance. Pour que cette conformité soit possible, il faut supposer une disponibilité naturelle de l'intention à vouloir le bien, mais sans qu'elle soit déterminée à le vouloir avant de le saisir.

S'il est donc nécessaire, dans la condition imparfaite de l'homme, de recourir à l'idée d'obligation, c'est premièrement pour le conduire à découvrir l'existence de la valeur et, dans le cas où cette connaisance n'est pas immédiate, de le forcer à la désirer dans l'aveuglement, en passant par la norme qui est supposée l'exprimer. Qu'elle soit distante, et même voilée, c'est toujours dans la valeur que se trouve l'origine de l'obligation. Car si la valeur se présentait de façon immédiate et éclatante, l'obligation deviendrait superflue. Scheler le dit de la façon suivante: «Si je sais immédiatement et pleinement ce qui est bon, ce savoir affectif détermine également et de façon 
immédiate mon vouloir, sans que j'aie besoin de passer par l'entremise d'un "je dois".»"

Ni l'une ni l'autre de ces deux perspectives ne peut nous indiquer s'il existe réellement une obligation à la valeur et, en fin de compte, ni une morale exclusivement formelle ni une morale exclusivement matérielle ne peuvent nous dire si, et en quoi, nous sommes obligés envers autrui. Mais dans la perspective d'une morale prise globalement dans ses deux dimensions, la valeur n'est ni une «réalité-en-soi», totalement indépendante du sujet et de son engagement personnel, ni une simple projection imaginaire de la subjectivité qui ne concernerait pas autrui. La valeur n'est donc ni une réalité sui generis, comme l'a proposé Emile Durkheim ${ }^{12}$, ni le contenu d' un être des valeurs comme le décrit Max Scheler, sans spécifier très précisément le rapport qu'il y a entre la valeur et l'être. Par contre, comme nous avons montré qu'il y a un lien essentiel entre la valeur et le sujet, nous croyons qu'Eugène Dupréel a raison de proposer d'aborder l'étude de la valeur par l'idée de précarité. «La valeur est précaire par le fait qu'il n'y a de valeur que par un sujet». ${ }^{13} C^{\prime}$ 'est cet ensemble de concepts qui nous permet de dégager le rapport spécifique qui existe entre la valeur et l'être, et qui nous permet de préciser la signification particulière de la valeur morale et de son rapport à la société.

En distinguant, comme nous l'avons fait plus haut ${ }^{14}$, les jugements d'estimation des jugements de valeur, selon l'argumentation proposée par Durkheim, nous avons rejeté leur assimilation car nous pensions que les jugements d'estimation n'exprimaient que des «états déterminés du sujet», et non une relation objective rattachée à un idéal. Toutefois, si nous poursuivons cette idée et reconnaissons qu'il n'y a de valeur que par le sujet, nous admettons que la valeur doit être à la fois objective et subjective pour être valeur, c'est-à-dire qu'elle doit comprendre une réalité extérieure qui ne soit pas le sujet, et un état intérieur qui ne puisse se comprendre que par lui. Voilà pourquoi la valeur est précaire, car elle tente de concilier, dans les conditions de son existence, deux aspects logiquement et conceptuellement inconciliables. Car elle ne peut être assimilée, en effet, ni à l'une ni à l'autre des deux réalités auxquelles elle doit être essentiellement rattachée pour être. Il n'est donc pas possible de parler de l'être de la valeur, comme si elle avait un être propre; puisqu'elle ne peut exister que par autre chose qu'elle. Il en sera ainsi de la société-sujet qui incarne la valeur de la moralité.

Chaque fois que nous parlons de la valeur, nous parlons des deux êtres par lesquels elle existe, à savoir le sujet et une réalité objective. Dire d'un objet qu'il «a» de la valeur, un bijou (richesse), un tableau (beauté esthétique), nous reconnaissons une valeur «dans» ces objets, mais nous ne disons pas

\footnotetext{
11 lbid., p. 225

12 Emile Dirkheim, loc. cit., p. 100

13 Eugène Dupréel, Esquisse d'une philosophie des valeurs, p. 91

14 Voir p. 124
} 
que ces objets «sont» eux-mêmes la valeur. Nous dirons qu'ils sont des objets porteurs de valeur, réalité objective dans laquelle la valeur se manifeste. Mais comme ces objets ne sont pas «en soi» des valeurs, ils ne peuvent être réellement porteurs de valeurs que s'ils sont reconnus comme tels. Le bijou n'a de valeur que pour celui qui le possède, ou celui qui veut l'acquérir, dans une société qui accorde une valeur à ce bijou. Un arbre en fleurs n'est beau que s'il est vu et admiré; il n'est pas beau en lui-même. C'est donc toujours par rapport à des sujets qui attribuent une valeur aux choses que celles-ci ont de la valeur. L' homme est donc un être «attributeurde-valeur», ou créateur de valeur comme nous l'avons si souvent répété plus haut, sans qui la valeur n'existerait pas dans la réalité. C'est donc toujours "pour» l'homme qu'il y a des valeurs «dans» les choses.

Nous rencontrons ici une des objections fondamentales à l'éthique de la prospérité, qui fait de l'accroissement de la richesse la valeur motivante de la morale. Car la richesse n'est pas une chose en soi, elle n'est que la quantité qui s'attache aux moyens du bien-être. Or si les choses que la richesse permet d'acquérir peuvent êtres elles-mêmes porteuses de valeur, car elles sont choses valables «pour» l'homme, ce n'est pas en augmentant leur quantité que l'on en augmente la valeur. La quantité est chose en soi, elle n'est pas «pour» moi, donc ne peut avoir ce caractère de la valeur. L'homme ne crée donc pas de valeur en augmentant indéfiniment les moyens de son bien-être. S'il croit le faire, il s'illusionne.

Cette création des valeurs n'est cependant pas ex nihilo, car il n'y a pas de valeur possible sans la réalité d'un objet auquel l'homme attribue des valeurs. L'homme est source d'une transformation valorisante de la réalité, mais il n'est pas la source de l'être des valeurs, car l'homme ne crée pas l'objectivité du monde. C'est pourquoi nous dirons que la valeur est toujours «incarnée» dans les choses, dans des choses qui ne sont pas moi mais qui portent une valeur pour moi. Ainsi les choses existent sans moi, mais elles ne peuvent pas être belles ou bonnes sans moi. Parler de la valeur, c'est donc parler de la relation très spécifique qui lie l'homme aux choses, aux êtres qui ne sont pas lui mais qui ne valent rien sans lui. L'homme serait incapable de prendre conscience des valeurs si les choses ne lui donnaient pas la possibilité de les voir dans l'existence; mais les choses n'auraient pas de valeur en elles-mêmes sans leur relation avec l'homme. Il est donc juste de dire, à la façon de Sartre: «l'homme est l'être par qui la valeur vient aux choses». Nous voyons déjà, à ce stade de notre raisonnement, comment se dessine la différence fondamentale que nous allons faire entre la sociétéobjet, qui est donnée et qui existe, et la société-sujet qui résulte de l'action valorisante de l'homme.

Toutefois, la valeur n'en dépend pas de façon similaire. L'être des choses est, en soi, indifférent à la valeur; son existence ne dépend pas de sa relation valorisante avec l'homme. Il en est ainsi de la société-objet. Par contre, ce n'est pas accidentellement que l'homme attribue des valeurs au monde, mais essentiellement; cela lui est existentiel. Donner de la valeur 
aux choses, pour l'homme, fait partie de l'essence même du rapport de l'homme au monde. C'est pourquoi renoncer à attribuer des valeurs aux choses et au monde, renoncer à faire des choix de valeur, signifierait pour l'homme un renoncement à sa nature de sujet dans sa relation au monde, une fusion de son être dans l'objectivité de l'existence amorale. Car ce n'est finalement pas le monde de l'objet qui demande sa valorisation par l'homme, puisqu'il peut exister sans elle; c'est notre être même de personne en devenir qui exige cette valorisation du monde empirique. Ce n'est donc, finalement, que par rapport aux personnes humaines qu'il peut exister des valeurs; mais celles-ci sont toujours incarnées dans l'objectivité des choses, «pour» les personnes.

Parler d'une obligation à la valeur c'est dire qu'il existe un besoin de valorisation du monde par lequel nous devenons ce que nous sommes. Mais c'est aussi une obligation qui consiste à tirer le monde hors de l'absurdité d'une existence sans signification. Le monde existe, mais il n'a de sens que lorsque l'homme l'imprègne de valeur "pour» l'homme. Affirmer cela implique le rejet de toutes les théories morales selon lesquelles les valeurs sont inscrites dans les choses ou l'univers, dans une objectivité qui prétendrait être la valeur sans l'homme, là où l'homme aurait un devoir de la découvrir et de la déchiffrer. Nous croyons au contraire que l'homme ne découvre pas les valeurs en dehors de lui, préalablement à sa décision, mais qu'il les invente à l'image de son propre devenir, c'est-à-dire à l'image de la personne. C'est pourquoi l'obligation à la valeur est une obligation à la personnalisation du monde, en d'autres mots à la transformation des choses en "choses pour les personnes".

\section{La société est la première de ces réalités du monde que l'homme a l'obligation de "personnaliser».}

C'est pourquoi le rapport du sujet à la communauté des sujets peut être interprété maintenant dans le cadre du rapport de la personne et de la société. Et tandis que nous avons vu comment la société-objet, donnée objective de la condition humaine, ne créait, par la contrainte sociale, qu'une illusion de l'obligation, ce que nous appelons maintenant la société-sujet, celle que nous avons d'abord postulée, pour donner un sens à notre analyse, et que nous découvrons comme valorisée par un rapport spécifique à la personne, se présente comme une pierre essentielle dans la construction d'une morale dans laquelle la personne et l'objectivité de la communauté des personnes sont ensemble appelées, par un partage conscient de leur responsabilité réciproque, à un devenir digne de la destinée humaine.

Nous sommes enfin en mesure de comprendre l'origine de la contradiction qui se dressait entre la conception formelle de l'obligation et une conception matérielle qui déterminait la volonté par le bien. Au moment où nous acceptons que la valeur n'est pas autre chose que l'objectivité pour les 
personnes, c'est-à-dire une correspondance immédiate du contenu moral et de sa forme, par la notion même de personne, il devient équivalent de dire que la valeur est fondée sur l'obligation ou que l'obligation est fondée sur la valeur. Car toutes deux, valeur et obligation, ne se présentent plus comme des absolus rationnels enfermés dans leur ordre conceptuel propre, mais comme des fonctions morales destinées à l'accomplissement de la finalité ultime de la morale qu'est le devenir des personnes dans une société-sujet, responsable elle-même de ce devenir.

Ces notions apparaissaient contradictoires aussi longtemps qu'on tentait d'en faire des éthiques séparées qui s'achevaient dans une moralité construite pour satisfaire les exigences de la raison. Mais au moment où la recherche morale ne se limite pas à la construction de cette moralité, mais poursuit une amélioration de l'homme lui-même, alors la contradiction disparaît. Dire que la valeur se fonde sur l'obligation, c'est reconnaître que le contenu moral doit s'informer, devenir malléable, se conformer aux exigences du devenir formel de la personne. Dire d'autre part que l'obligation se fonde sur la valeur, c'est indiquer que la personne ne peut devenir elle-même que par le Bien, c'est-à-dire en communauté avec d'autres personnes, par la construction d'une société valorisante dans une objectivité respectueuse des personnes. Nous pouvons l'appeler la solidarité humaine. Nous pourrons enfin comprendre qu'affirmer l'existence d'une obligation à la valeur signifie tout simplement la reconnaissance de la valeur de l'obligation. Ceci revient à en reconnaître l'efficacité et la nécessité pour la personne. Car il n'y a de valeur que des personnes, et c'est pour la personne que valeur et obligation se conjuguent dans le devenir moral.

\section{L'obligation pour autrui et l'obligation sociale}

En analysant plus haut la relation de hiérarchie qualitative qui existait dans le jugement de valeur, nous avons indiqué que la valeur ne pouvait être considérée comme une notion simple exprimant le bien ou le mal, mais comme l'expression d'une préférence, c'est-à-dire d'un choix par rapport à d'autres valeurs. Il n'est donc pas possible d'isoler une valeur et de la considérer en elle-même, elle se présente toujours en relation avec d'autres valeurs, soit des valeurs négatives, soit des valeurs inférieures, soit une valeur idéale qui demeure comme un absolu au delà de la valeur elle-même. Si les valeurs, le bon, le mal, le beau, étaient des notions simples, comme celles des couleurs, le jaune ou le rouge par exemple, elles ne pourraient être d'aucun secours dans l'analyse morale, car elles seraient inanalysables et inexplicables ${ }^{15}$. Mais c'est justement parce qu'elles ont, dans le domaine

15 C'est George Edward Moore, dans ses Principia ethica (1903), qui a souligné la difficulté de la transmission des ces notions simples. Comment en effet expliquer le «jaune» à quelqu'un qui ne l'a jamais vu. Il en est, dit Moore, de même pour la notion de bien. 
moral, une relation indispensable avec la personne qu'elles peuvent apporter quelque lumière à notre recherche.

En acceptant l'idée proposée par Scheler dans son analyse de la notion de personne, qui dit que "ce n'est qu'à partir d'un certain niveau d'existence humaine que le concept-de-personne trouve son application", ${ }^{16}$ et en nous appuyant sur ce que nous avons affirmé, à savoir qu'il n'y a de valeur que de la personne, nous dirons que la hiérarchie des valeurs que nos choix établissent n'exprime pas autre chose que le devenir même de la personne, saisi à travers les différents niveaux de personnalisation de l'existence. Ainsi, affirmer une valeur signifie, non pas seulement préférer une valeur par rapport à d'autres, mais choisir un niveau de développement de la personne dans l'évolution de l'existence humaine. C'est pourquoi ce choix, dans la mesure où il est authentique - et non pas voilé par un abandon à la contrainte - demande un engagement total et existentiel. Le corollaire de cette affirmation implique que rien ne demeure sans valeur dans la mesure où la personne en fait l'objet de son choix.

C'est en ce sens qu'il faut comprendre ce qu'Aristote, et à sa suite Thomas d'Aquin, entendaient quand ils disaient que la volonté ne pouvait vouloir que le bien. Cela signifie qu'elle ne peut pas vouloir une fin qui ne lui apparaîtrait pas comme valeur. Sartre ne dit pas autre chose: "Choisir d'être ceci ou cela, c'est affirmer en même temps la valeur de ce que nous choisissons, car nous ne pouvons jamais choisir le mal; ce que nous choisissons, c'est toujours le bien». Et il ajoute aussitôt: «et rien ne peut être bon pour nous sans l'être pour tous». ${ }^{17}$

Nous parvenons ici au coeur de notre raisonnement. Du fait que le choix moral a toujours lieu dans une action, que cette action est destinée à être manifestée à autrui, le choix personnel d'une valeur propre - et il est impossible d'en choisir une autre, car, comme l'écrit Aristote, «le vrai bien est individuel et impossible à enlever à son possesseur, ${ }^{18}$ - est un choix qui concerne autrui, la société, tous les hommes, et par là devient universel. Voilà pourquoi, chaque fois que le sujet fait un choix, et qu'il affirme ainsi une hiérarchie des valeurs, non seulement il choisit une valeur qui exprime son propre devenir personnel, mais il affirme également, par son intervention, le devenir personnel de tous. Si l'obligation demande à l'homme de devenir une personne, pour répondre aux exigences de son être propre, elle lui demande également, en raison même de la relation morale qui le lie à autrui, d'affirmer dans ses valeurs et d'assurer dans son action le devenir moral et personnel de tous les hommes. Ainsi l'obligation à la valeur devient une obligation à la valeur pour autrui.

\footnotetext{
16 Max Scheler, op. cit., 477

17 Jean Paul Sartre, L'existentialisme est un humanisme, p. 25

18 Aristote, Ethique de Nicomaque, Livre I, Chap. V, 4.
} 
Cette obligation pour autrui n'est en fait pas autre chose que l'obligation envers la communauté des autres personnes, c'est-à-dire envers la société. Mais cette société à laquelle l'obligation appelle n'est plus la société-objet qui pèse de tout son poids sur les individus qui la composent et qui est la source de la contrainte, mais une société transformée par le devenir personnel de ses membres; elle est en quelque sorte personnalisée par l'action morale et la valeur, voilà pourquoi nous l'appelons une société-sujet.

La première conséquence à en tirer, dans la logique des implications de l'obligation, est qu'il ne peut y avoir de distinction, et même de séparation, entre l'obligation morale envers soi-même et l'obligation envers la société; en d'autres mots, il n'est pas possible de séparer l'éthique individuelle de l'éthique sociale, la morale de la personne de la morale du groupe. Car si la valeur exige que «rien ne puisse être bon pour nous sans l'être pour tous», il n'est pas possible d'imaginer que les valeurs régissant la morale des individus puissent être différentes des valeurs dominantes du groupe social. La société-sujet ne peut admettre que la morale soit considérée comme une affaire privée, et que la société soit considérée séparément et puisse fonctionner selon des principes et des valeurs totalement différentes. Il n'y a donc pas une logique de l'éthique de la prospérité qui puisse prétendre mettre en cause la logique de l'obligation personnelle. Même dans le plus grand secret de sa vie individuelle, le choix de valeurs engage toujours toute l'humanité. Et toute disposition qui résulte du fonctionnement de la société implique le devenir individuel de toutes les personnes qui y vivent. Nous pourrions dire que le sommet de l'acte immoral, dans cette perspective, est de vouloir faire croire que le fonctionnement de la société est amoral. La société morale, c'est-à-dire personnalisée, est donc bien dans un certain sens, un sujet authentiquement responsable. Il n'est nullement envisageable de mettre sa responsabilité entre parenthèses dans quelque situation que ce soit..

Car l'obligation envers la société est de telle nature qu'elle exige une forme d'obligation «de» la société, c'est-à-dire des personnes prises comme groupe, envers chacune des personnes individuelles qui y sont en devenir. Mais cette obligation «de» la société doit revêtir les mêmes caractéristiques que l'obligation du sujet, bien que la société ne puisse être ontologiquement assimilée à un sujet. Cette obligation ne doit donc pas être conçue comme une obligation préétablie, fondée sur une hiérarchie des valeurs préalablement posée aux choix individuels. C'est finalement chaque personne qui doit assumer l'obligation de tous. C'est le sens même de ce que nous appelons une responsabilité partagée.

Car la valeur est toujours issue de ce qu'il y a en nous de plus profond et de plus intime. Il doit donc y avoir correspondance de la valeur et du moi profond, au delà même de la contrainte sociale et au delà de la rationalité universelle, c'est pourquoi chaque personne qui doit assumer l'obligation «de» la société ne peut en aucun moment renoncer à son engagement subjectif sans nier la valeur. Car si cette relation entre la valeur et le moi 
profond est si étroite, c'est justement parce que la valeur doit correspondre à ce que je dois être pour être une valeur, et que mes choix ne peuvent jamais être ceux d'autrui sans la renier. Assumer l'obligation «de» la société est toujours un acte individuel, quel que soit le niveau de responsabilité formelle auquel je me situe; c'est la forme concrète et permanente de mon obligation envers autrui.

Mais, comme si l'obligation du sujet envers la société se reflétait dans un miroir, il existe une obligation correspondante du groupe en tant que tel, c'est-à-dire une obligation collective de la société envers les personnes. Chacun pourrait l'exprimer en disant: «envers moi». Ceci est le cas dans tous les groupes de personnes, qu'ils soient spontanés, volontaires, organisés ou étatiques. Du plus petit chef de bande aux hommes d'Etat le plus hautement placés, de l'animateur des plus petits groupes humains aux plus grands chefs d'entreprises, des organisateurs spontanés aux plus puissants gestionnaires d'organisations, l'obligation «de» la société, celle que toute société doit assumer vis-à-vis de ses membres, - car ils sont des sujetspersonnes en devenir - doit être assumée et partagée par chacun. Cette obligation sociale, toutefois, qu'elle soit assumée par un homme d'Etat ou un modeste employé dans une entreprise, demeure une obligation personnelle. Aucun sujet ne peut renoncer à son être de sujet dans l'action, même s'il agit au nom du groupe. Personne ne peut jamais se réfugier derrière la neutralité d'une fonction, que ce soit la "raison d'Etat» pour les princes de cette terre, ou la fonction et les ordres pour les exécutants, car c'est en chacun que s'incarne l'obligation "de» la société. A chaque niveau des hiérarchies institutionnelles ou fonctionnelles, de l'Etat, de l'économie ou de la vie sociale, l'homme, quelle que soit sa fonction, demeure «celui par qui la valeur vient au monde». L'obligation collective n'est donc pas une contrainte impersonnelle, car elle n'existe que par les sujets qui l'actualisent. C'est ainsi que la société-sujet peut se gagner ou se perdre.

C'est pour cette raison que l'affirmation de l'existence, dans la société, ou même transcendant la société, d'une hiérarchie des valeurs, objective, fondamentale et suprême, fondée sur une sorte de valeur critère unique par rapport à laquelle toutes les hiérarchies de valeurs devraient être jugées, mettrait en danger l'existence même de la valeur. Il n'y aurait plus de société-sujet, et le devenir des personnes serait mis en danger. En effet, cette valeur ne serait plus une valeur par rapport au sujet qui s'engage en son nom, mais valeur par rapport à un critère purement objet, que celui-ci soit le Bien en soi de la philosophie, le Dieu des chrétiens ou la Foi des religions politiques, la Finalité de l'histoire ou la richesse des sociétés capitalistes. Car comment pourrais-je, en tant qu'homme, accepter la légitimité d'une société qui affirmerait des valeurs par rapport auxquelles je ne pourrais engager ni ma liberté ni ma responsabilité. Car si je ne demeure pas moimême, avec toute ma subjectivité, comme membre de la société dont je suis prêt à assumer l'obligation, je me renie en tant que personne. Ce serait, à la limite, une forme de suicide. 
$\mathrm{Si}$, par contre, la valeur n'a pas d'autre fondement que celui que je veux bien lui donner par les préférences que j'établis, et l'engagement par lequel je me manifeste au monde et à autrui, et surtout si je le fais au nom de la société dans laquelle je trouve avec autrui les conditions de mon devenir, alors la valeur peut être authentiquement, pour moi comme pour tous les hommes, l'occasion d'une invention responsable.

Mais il ne faut pas, pour cela, que la valeur soit assurée d'avance car l'obligation sociale deviendrait alors l'occasion de la détourner à notre profit et d'échapper ainsi à notre obligation envers autrui. Dupréel l'écrit très justement: «Persister à voir dans un Être infini et parfait l'expression de la valeur, ce serait faire de celle-ci une grandeur exempte de toute vicissitude. Pour chacun de nous le souci majeur ou exclusif serait, non d'augmenter ou de maintenir ce maximum assuré, mais de l'exploiter à notre profit. Si la valeur est une grandeur assurée, mon mérite n'importe qu'à moi seul, non à l'Univers. Si la valeur est une quantité variable, mon action est capable de l'augmenter ou de la réduire: mon mérite devient une affaire qui intéresse l'Univers." ${ }^{19}$ Comme nous le verrons au Chapitre 8, il en est de même de la Justice.

C'est justement parce que la valeur est précaire et qu'elle dépend de mon libre choix et de mon engagement, qu'elle renferme, dans mon obligation envers autrui, la possibilité de contribuer au devenir de la société par une responsabilité collective partagée. Si la valeur était établie et ne dépendait pas de moi, si le devenir des personnes était inscrit dans le développement du monde, de l'histoire et des sociétés humaines sans que des individus puissent en détourner le cours, je ne pourrais affirmer la valeur que dans l'espoir d'exploiter le devenir de l'univers au profit de mon propre devenir. Combien d'usurpateurs n'ont-ils pas fait une telle main basse sur le devenir du monde en s' attribuant le pouvoir de l'Etat? Combien de profiteurs n'ontils pas détourné des richesses à leur propre profit soi-disant parce qu'elles auraient pu demeurer inutilisées? C'est ainsi que dans l'éthique de la prospérité, l'enrichissement individuel, valeur reconnue, peut se faire au détriment d'autrui. La redistribution n'est pas nécessairement le partage. En d'autres mots, si le bien est donné d'avance, la bonté morale n'aurait pas d'autre but que de sauver mon âme.

Mais si une telle valeur suprême n'existe pas, je deviens responsable des valeurs que je propose à autrui, et le mérite de mon action l'intéresse. J'ai donc envers lui l'obligation de vouloir le bien, car mon devenir est condition de son devenir dans une responsabilité sociale partagée. La personnalisation de mon existence devient indispensable à celle de son existence. Nous devenons solidaires dans notre insécurité. L'obligation envers autrui n'a donc pas besoin de faire appel à l'idée d'une communauté de destin, d'une

19 Eugène Dupréel, op. cit., p. 103 
nation ou d'une société qui nous dépassent et dont la finalité commune, non choisie, nous imposerait des devoirs réciproques, mais sur le fait que le devenir de la personne d'autrui dépend de moi et de ma création de valeurs, comme je dépends moi-même de la responsabilité sociale qu'il veut bien assumer. Il y a finalement une impossibilité de valoriser exclusivement mon existence propre sans impliquer obligatoirement la valorisation d'autrui et de la société.

C'est pourquoi cette obligation envers autrui, créatrice de la sociétésujet, ne se distingue pas et ne s'ajoute pas à l'obligation envers soi-même, mais en fait essentiellement partie. Il n'est pas possible de devenir soi-même d'abord, pour ensuite s'occuper du devenir de l'autre et de l'obligation sociale. "A aucun moment une conscience n'est capable d'un accroissement d'être, qu'elle n'en soit redevable, tout d'abord, à son dialogue avec une autre consciencen. ${ }^{20}$ La réalité de l'obligation se trouve donc dans la prise de conscience que ce sont le(s) personne(s) qui sont le devoir être. Ma personne et celle d'autrui dans une même affirmation de la valeur qui, par l'action morale, est créatrice d'une société confirmant les personnes dans leur devenir. Il n'y a finalement qu'une seule obligation. Non pas une obligation envers moi-même s'ajoutant à une obligation envers autrui, qui équilibreraient entre elles, plus ou moins bien, l'opposition de leurs finalités respectives, mais une seule obligation de nature sociale qui m'oblige au devenir de moi-même par autrui, et au devenir d'autrui par moi-même.

La personne ne devient elle-même que dans une relation sociale avec autrui, obligée de viser l'être d'autrui dans le même développement moral par lequel elle est pour elle-même la fin de son propre devenir. Ce n'est donc pas à un principe que se résume toute la morale, mais à des êtres existants, des êtres concrets, sujets et fins de l'obligation qui les appelle à leur propre devenir. Tout devient dès lors relatif par rapport au devenir des personnes: les maximes morales, les principes formels, l'amour lui-même et le devoir.

Au delà de l'éthique elle-même, c'est le devenir de l'homme comme personne qui est visé. La morale devient donc situationniste, puisqu'elle est à reconstruire en permanence selon l'évolution des personnes, selon leur rencontre et leur situation. Ce qui peut guider l'agent moral, à travers la variation des situations morales concrètes, ce n'est pas un principe formel érigé en maxime absolue, c'est la conscience de la personne qui demeure permanente et qui lui dit que, quoi qu'elle fasse, quel que soit le principe auquel elle obéit, quelle que soit la finalité particulière qu'elle poursuit, elle demeure responsable de tout ce qu'elle devient et de toutes les personnes qu'elle rencontre. Finalement, il n'y a ni loi, ni devoir, ni principe, ni amour même, qui puisse s'intercaler entre les personnes et déterminer la nature de l'authentique rencontre par laquelle elles gagneront leur être propre. Une éthique personnaliste nous interdit donc de parler d'une obligation à la

20 Jean Nabert, Eléments pour une éthique, p. 169 
valeur, à l'amour même, car c'est toujours au delà des valeurs, au terme de l'amour, que nous rencontrons dans l'existence réelle la source de toute morale et la réalité fondamentale de toute éthique: les personnes et leur devenir.

Est-il encore possible de parler à ce niveau d'une obligation morale? On comprend maintenant que ce n'est pas en fonction d'une éthique particulière que l'obligation trouve sa signification définitive, mais à partir de l'homme. L'obligation est donc avant tout une obligation humaine. Elle donne dès lors un sens à la vie, et plus particulièrement à la vie sociale. C'est elle qui nous oblige à nous dépasser pour être homme, à nous chercher toujours au delà de nos propres limites, à refuser la tentation de repos dans l'être-objet que la contrainte nous impose, à faire éclater les entraves à notre liberté, et à construire par l'appel aux valeurs une société dans laquelle le moi ne dit authentiquement «je» qu'en reconnaissant aussitôt que c'est un «nous» qui l'a fait naître à la vie et à l'être. L'obligation envers soi, l'obligation envers autrui et l'obligation sociale trouvent ainsi leur signification définitive dans une existence transformée par une obligation envers l'homme. 


\section{Chapitre 7}

\section{Le retour du sujet responsable}

Il est impossible d'avoir une prospérité générale et durable sans un certain degré de moralité publique.

Henri Hude Ethique et politique

Seul l'appel au sujet fait retrouver la distance au marché qu'implique tout jugement moral, et permet de reconstruire ce que la société de consommation décompose.

Alan Touraine Critique de la modernité

La thèse principale ce livre apparaît maintenant claire. L'obligation morale sert de fondement à une rénovation de l'éthique qui vise à situer dans le sujet, dans l'homme en tant que personne en devenir, la responsabilité ultime de la moralité sociale. Cette rénovation tient compte de deux choses essentielles. D'une part de l'héritage de notre civilisation, sans lequel les idées proposées ne seraient pas porteuses de sens et demeureraient étrangères au langage éthique que nous sommes capables d'appréhender, - d'où l'enracinement de cette tentative dans l'histoire de la philosophie - et d'autre part des conditions del'action humaine et de la vie morale contemporaine, qui exigent une intervention renouvelée de la réflexion éthique dans les domaines de l'économie et de la politique en cette fin du XXe siècle.

Cette intervention de la philosophie doit répondre aux défis contradictoires de ce que nous appelons l'éthique de la prospérité, qui se présente comme 
une éthique cohérente susceptible aujourd'hui d'entraîner l'adhésion de la majorité, et qui s'adresse avant tout à la dimension matérielle de l'activité humaine. Nous avons rapidement décrit, dans le premier chapitre, la situation historique dans laquelle cette interrogation se situait. Nous souhaitons maintenant, après avoir analysé la nature et la profondeur existentielle de l'obligation morale, mettre en évidence les enjeux des questions que cette éthique soulève.

\section{L'éthique de la prospérité: paradigme et contrainte}

L'éthique de la prospérité est une morale de l'enrichissement matériel immédiat, tant de la société que des individus. Liée à une pensée économique récente que les derniers succès du capitalisme ont rendue incontournable, cette éthique se présente comme une évidence pratique, donc morale, qui prétend contenir en elle-même les preuves de sa rectitude. Fondée sur une conception productiviste de la société qui se justifie par son propre succès, elle prône une idéologie de la croissance qui doit aboutir à la satisfaction des besoins de l'homme par l'accroissement illimité de ses richesses. Mais contrairement à une éthique du devoir qui n'a pas d'autre fondement que l'obligation elle-même, «tu dois parce que tu dois», l'éthique de la prospérité s'appuie sur une logique déduite des faits concrets et visibles, ceux qui résultent du succès et de l'efficacité indéniables de l'économie contemporaine. Elle est donc typique d'une société-objet.

L'abondance est une des caractéristiques évidentes des sociétés occidentales contemporaines. Comme les historiens de l'économie l'ont très souvent souligné, elle est le résultat des transformations technologiques et sociales qui ont marqué l'évolution de nos conditions de vie et de nos mentalités durant ces deux derniers siècles, avec l'industrialisation et le développement du capitalisme. Plus récemment, encouragées par les succès de la croissance économique qui a suivi la crise des années trente et les désastres de la deuxième guerre mondiale, et qui a permis de faire disparaître de l'éventail des événements prévisibles la menace endémique de la misère et de la pauvreté, nos sociétés occidentales ont poursuivi des politiques économiques bien spécifiques ayant pour but de maintenir et d'accroître une prospérité devenue non seulement possible, mais réelle. Même si, dans son célèbre ouvrage de 1958, The Affluent Society, John K. Galbraith a montré que les sociétés d'abondance continuaient à vivre et à penser dans un cadre d'idées et de principes hérité de l'expérience passée, où la pauvreté était la condition normale de la majorité, nous nous sommes habitués, depuis les Trente glorieuses ${ }^{1}$, à n'imaginer l'évolution de nos sociétés qu'en termes de croissance régulière, d'amélioration constante du niveau de vie et de progrès continu de l'économie.

1 Jacques Fourastié, Les Trente glorieuses 
Ce résultat n'est pas fortuit, et les explications socio-historiques qui rattachentl'évolution du monde occidental à ses valeurs éthiques et religieuses sont suffisamment nombreuses pour qu'il ne soit pas nécessaire de les répéter ici. La thèse de Max Weber sur L'éthique protestante et l'esprit du capitalisme est connue; elle n'est contestée que sur certains de ses aspects et non sur le fond ${ }^{2}$. La prospérité occidentale n'est donc pas un hasard, et l'éthique qui en est à l'origine couramment reconnue. Comme le dit Henri Hude, la prospérité exige une moralité; elle «est toujours à base de confiance, de travail, d'épargne et d'investissement, d'esprit d'initiative et d'entreprise» ${ }^{3}$.

Mais si la morale sociale et religieuse de l'Occident explique en partie la naissance du capitalisme, l'industrialisation récente et la prospérité de notre époque, l'existence et le déploiement de cette dernière entraînent à leur tour une transformation des comportements individuels et sociaux, ainsi que des valeurs qui les sous-tendent, au point de donner naissance à une éthique qui n'a plus rien à voir avec celle qui en explique ses origines, celle que nous appelons ici l'éthique de la prospérité. Il ne s'agit pas de l'éthique sociale qui rend la prospérité possible, au sens du raisonnement wébérien, mais celle que la prospérité économique produit, et finalement impose, pour garantir sa propre survie et se développer. Il s'agit dans ce cas d'une véritable contrainte morale.

Cette éthique de la prospérité est avant tout le produit du fonctionnement de l'économie elle-même. En effet, celle-ci a accumulé récemment de tels succès de croissance qu'ils ont engendré une civilisation qui n'a plus à se justifier par l'appel à d'autres valeurs extérieures ou transcendantes, mais qui trouve son sens dans la satisfaction matérielle que son propre fonctionnement procure. Il y a donc une rectitude de l'action, une forme de vertu, qui se fonde sur l'efficacité des structures et des processus par lesquels individus et sociétés sont capables d'augmenter leurs richesses.

Même si cet accroissement de richesse peut concrètement servir à une libération de l'homme, celle que procurent la maîtrise des contraintes physiques et la satisfaction des besoins fondamentaux de la vie, - logement, nourriture, santé, etc., - la logique de la prospérité et de sa croissance entraîne des conséquences sur le fonctionnement de l'économie qui bouleversent radicalement le comportement des producteurs et consommateurs, au point de transformer les valeurs des acteurs économiques eux-mêmes. Galbraith appelle cela "l'effet de dépendance" (The Dependence Effect), celui qui consiste à reconnaître que la satisfaction des besoins humains, qui justifie la production, est en même temps la source de la stimulation de ces besoins. "Les besoins sont, passivement et volontairement, les fruits du processus par lequel on tente de les satisfaire. ... la production induit davantage de besoins et, en conséquence, un besoin supplémentaire de production. On a

2 Voir par exemple: Michel Istas, Les morales selon Max Weber

3 Henri Hude, Ethique et politique, p. 30. 
jusqu'à maintenant, par un tour de force important, ignoré les conséquences de ce phénomène. Mais il est évident que c'est une solution dangereusem. ${ }^{4}$

C'est ainsi que l'économie contemporaine, par ses succès et son pouvoir, a radicalement transformé la vie sociale et les valeurs sur lesquelles elle repose. Les activités économiques et les structures dans lesquelles elles $s$ 'incarnent sont non seulement reconnues comme déterminantes pour la vie des sociétés humaines, mais ses acteurs ne donnent plus l'impression d'être capables de maîtriser les processus mis en route. Il semble que, par un phénomène d'auto-stimulation incontrôlé, les activitéséconomiques entraînent des comportements qui n'ont d'autre finalité que l'activité économique ellemême. Devenue essentielle à la survie des sociétés, l'économie pèse dès lors de tout son poids sur les comportements individuels. La société-objet productrice de richesses utilise la contrainte pour satisfaire ses propres fins.

Ceci est particulièrement le cas dans les grands pays dominés par les complexes militaro-industriels qui s'enrichissent par la production d'équipements dont on n'espère jamais se servir. Ce n'est plus l'économie qui est mise au service de la défense et de l'armement, c'est ce dernier qui, artificiellement protègé et maintenu, même sans utilité immédiate, permet à l'économie de fonctionner. D'autres exemples abondent par lesquels il est possible de montrer comment l'activité économique est ainsi devenue une fin en soi. Or, comme ce sont les finalités sociales qui justifient la contrainte diffuse et organisée, nous comprenons comment les visées utilitaires qui sous-tendent l'éthique de la prospérité présentent un aspect parfaitement contraignant.

La contrainte que fait peser cette éthique sur les individus se fonde sur un sophisme qui contient toute la force de conviction du système. $\mathrm{Ce}$ sophisme, dit Michel Albert, "est à l'oeuvre dans l'enseignement du système économique érigé en principe directeur de la société: ce qui réussit est efficace, ce qui est efficace est vrai, donc ce qui réussit est vrai». Dans le vocabulaire de la philosophie pratique on pourra dire: ce qui réussit est bon. Le bien se trouve donc dans la performance; le mal dans l'inefficacité. La question des buts ne se pose pas.

La force contraignante du système, qui provoque à long terme une uniformisation progressive des comportements individuels, est fondée sur la cohérence d'un système qui justifie la rectitude de ses buts parl'efficacité de son action et de ses moyens, la satisfaction subjective des agents concernés, consommateurs et producteurs, le tout confirmé par des preuves indéniables d'ordre quantitatif. Car la croissance est quantifiée: il n'est donc pas possible de la mettre en doute. De plus, comme il est impossible de nier la supériorité du plus sur le moins, ou de mettre en cause la préférence qu'il

4 Galbraith, The Affluent Society, p. 136

5 Michel Albert, Capitalisme contre capitalisme, p. 231 
faut accorder au progrès sur la stagnation, toute argumentation contraire se détruit elle-même. Il faut être de mauvaise foi pour nier l'évidence.

L'évidence première est la dêpendance directe que l'on établit entre la vérité économique, celle qui garantit la prospérité, et le système capitaliste et l'écononie libérale de marché. La preuve en est donnée par l'échec historique de son contraire, l'économie étatique planifiée du communisme. Il y a donc, prouvée par l'histoire, une nécessité d'accepter les prémisses et la rigueurdu syllogisme. Ces prémisses affirment que l'activité économique a pour but de produire de la richesse, que celle-ci se mesure à l'augmentation des moyens économiques dont on dispose, - et par conséquent de l'activité qui s'y rattache - et que cette augmentation est non seulement un but en ellemême mais le critère qui permet de juger de la valeur ou de la rectitude de la contribution de chacun au processus de l'ensemble.

Il existe donc une logique, un langage et une forme de raisonnement des sociétés prospères qui conditionne chacun à se laisser convaincre - ou contraindre - de participer par son action individuelle à l'augmentation de la prospérité gênérale. Il est donc logique d'accorder une valeur morale au succès économique de l'action, c'est-à-dire au profit, car c'est lui qui justifie tout. Dans les théories du néo-capitalisme, quí poursuivent cette logique jusqu'à l'extrême, le profit se présente à la fois comme le but et le moyen de toute initiative et de toute morale. Michel Albert écrit: «Le succès du culte du profit dans les années quatre-vingt se mesure à la multiplication de ses sanctuaires. Jamais on n'a construit autant de business schools dont les livres sacrés commentent une même vulgate symbolisée par le Prix de l'excellence ... L'excellence pourquoi faire? Du profit, pardi! Et du profit pourquoi faire? Surtout ne posez pas cette question, car vous seriez immédiatement exclu du sanctuaire pour avoir mis en doute l'article premier du nouveau credo: la finalité du profit est le profit. Là-dessus on ne transige pas. L'impératif catégorique est d'évacuer la question "philosophique» de la finalité pour se cantonner dans l'étude technique des moyens"* L'accumulation du capital et de la richesse, qui n'a pas d'autre but que d'augmenter le capital et la richesse, est donc philosophiquement et éthiquement fondé sur le succès même avec lequel elle progresse. C'est par rapport à ce but que toute action humaine trouve sa signification et sa valeur.

La question de la relation du travail et du capital, qui a hanté la pensée économique depuis plus d'un siècle, a été définitivement tranchée au bénéfice de la valorisation quasi exclusive du capital. C'est lui qui permet de mesurer le profit. Car le travail, c'est-à-dire la contribution individuelle au processus global d'enrichissement, ne s'accumule pas dans le temps, et peut difficilement prétendre au statut de valeur finale aux yeux d'une éthique qui n'a de signification que par la croissance de la prospérité. Ce n'est donc plus dans son travail que l'individu trouve le sens de son action

6 Ibid., p. 230 
et le sens de sa participation sociale, mais dans la richesse qu'il accumule et dans sa contribution à l'augmentation du profit général. A la limite, ce but est suffisamment prégnant pour justifier moralement toute action humaine. Le nouveau décalogue pourrait ainsi être résumé: - en plagiant la formule de Saint Augustin ama et fac quod vis - «engendre du profit, et fais ce que tu veux!»

Disposant d'un langage qui trouve sa cohérence dans le choix de ses concepts, soutenue par une logique irréfutable, complétée par une contrainte qui s'impose aux individus par la force des faits, et renforcée par une adhésion non rationalisée des acteurs qui accompagne ce développement, cette éthique de la prospérité se présente comme un véritable paradigme de la société capitaliste et libérale. Or, son pouvoir de conviction ne se trouve ni dans la rigueur de ses notions ni dans la force de ses mythes, mais dans des faits quantifiables. L'éthique de la propérité se présente comme juste et vraie parce qu'elle est efficace et mesurable. La "richesse des nations», et particulièrement celle des nations libérales et capitalistes, est là pour le prouver.

De plus, les individus qui, dans les sociétés occidentales, se conforment le plus étroitement à cette contrainte de l'enrichissement, sont évidemment plus riches et plus puissants que les autres, prouvant par leur propre niveau de vie la rectitude palpable de l'éthique à laquelle ils se soumettent. Certes, il existe des systèmes sociaux différents, ainsi que des formes différentes de capitalisme, mais quelle que soit leur originalité, ils sont jugés à leur capacité d'engendrer du profit, indépendamment de la tolérance qu'ils accordent au respect des individus. La logique de la prospérité, soutenue par les lois de la concurrence, fait que les systèmes les moins efficaces, les moins compétitifs, sont entraînés par leur faiblesse relative à devenir de moins en moins efficaces, et sont à terme condamnés par l'histoire. La fin du communisme est là pour le prouver, et la catastrophe économique que sa disparition révèle renforce les arguments de ses détracteurs. Une société qui ne favorise pas le profit, et qui ne voit pas dans le profit le but du profit, en d'autres mots qui ne s'évalue pas elle-même à la quantité de richesses dont elle permet l'accumulation et qui ne vise pas avant tout l'augmentation des moyens de s'enrichir, est condamnée à disparaître. Le communisme, qui s'opposait à cette éthique, est mort: c.q.f.d.

On ne souligne pas assez la force contraignante d'un paradigme social et éthique dont la preuve se trouve dans les faits et non dans les croyances ou le raisonnement. Il semble que l'on assiste à une complète objectivisation des valeurs, puisque celles-ci sont devenues uniquement quantifiables. Comme nous l'avons vu, une telle objectivation devient à la limite a-morale. Peut-on dès lors en déduire que l'accumulation objective de richesse est elle-même a-morale?

Cette apparente indifférence de l'activité économique quantifiée par rapport aux valeurs subjectives crée en fait la force de la contrainte des 
sociétés d'abondance. Quel est en effet l'individu qui oserait se dresser contre l'évidence concrète et visible qui s'impose aux yeux de tous? Il semble donc, à première vue, que l'éthique de la prospérité a tous les atouts nécessaires pour entrainer l'adhésion universelle. On ose même envisager de construire un nouvel ordre mondial sur le modèle qu'elle propose!

Mais cette contrainte, qui vise à faire de l'individu un simple rouage d'efficacité dans la poursuite du but global de l'ensemble, réduit à l'évidence l'autonomie et la responsabilité morale de l'homme, qui est essentielle à la prise de conscience de sa vocation personnelle, et met ainsi en cause l'existence même de l'éthique. La force de conviction que représente le succès matériel des sociétés riches en cette fin de siècle a donc des limites. Quelles sont-elles?

\section{Limites de l'éthique de la prospérité}

L'éthique de la prospérité est avant tout une éthique finaliste. C'est ainsi que l'action humaine est jugée selon sa capacité ou son impuissance à atteindre la fin poursuivie. Comme nous l'avons vu, les morales fondées sur la finalité conduisent immanquablement à une certaine forme de déterminisme. La fin, aussi conforme à la raison qu'elle puisse être, se présente toujours, à l'instar de toutes les causes finales, comme une force supérieure et contraignante qui conduit infailliblement les êtres qui la poursuivent. L'agent devient donc secondaire par rapport au but qu'il vise; il perd son autonomie, il doit se conformer à des régles d'action qui s'imposent à lui avec toute la force de la nécessité; il est finalement réduit à l'état de simple moyen par rapport à la fin qu'il poursuit. Les morales finalistes réduisent ainsi considérablement le rôle de la liberté dans la construction éthique.

Certes nous avons distingué, plus haut, les fins qui sont données par la nature, Dieu, la société ou la métaphysique, sur lesquelles l'homme n'a aucun pouvoir, et celles que l'être humain peut choisir lui-même et par rapport auxquelles il exerce sa liberté. Or la pensée qui sous-tend les éthiques finalistes accepte l'idée que l'être humain trouve, dans l'action morale, l'accomplissement d'un destin qui est inscrit dans sa nature et auquel il ne peut échapper, même si ce destin lui demeure caché dans les conditions normales de la vie courante. Or, l'idée de destinée implique que l'homme ne peut devenir que ce qu'il est déjà. Les morales finalistes ont donc pour but de lui faire comprendre, et de l'aider à admettre, souvent contre le sentiment de sa propre liberté, qu'il a tout avantage à poursuivre les buts qui s'imposent à lui. Certes, toutes ces morales manifestent une croyance au bonheur, à une satisfaction finale, celle que chacun connaîtra quand il atteindra le but auquel il est destiné. De la vertu des stö̈ciens au bonheur des utilitaristes, on retrouve partout cette idée que la motivation morale se trouve dans la satisfaction des besoins de l'agent. Il suffit donc 
de se mettre à l'écoute de nos propres besoins pour trouver la voie morale que la nature impose.

Des idées similaires se retrouvent dans l'éthique de la prospérité. Reconnaissons tout d'abord que l'homme, devant la nécessité de satisfaire à ses besoins fondamentaux pour assurer sa survie, particulièrement s'il a connu les privations de la disette et le manque du minimum indispensable, peut être très facilement motivé à travailler pour augmenter sa propre richesse, et par là celle de la société dans laquelle il vit. C'est en ce sens qu'Henri Hude, cité plus haut, dit que la prospérité résulte d'une authentique éthique de l'effort, faite de travail, d'épargne et de peines. Mais ce qui caractérise particulièrement l'éthique née de la prospérité, c'est le critère au nom duquel l'efficacité de l'action est jugée. Il n'a en effet rien à voir avec le bonheur subjectif de l'agent, bien que celui-ci soit présupposé, mais consiste uniquement dans une mesure extérieure, la quantité de la richesse acquise. C'est ainsi que la notion de croissance a remplacé, dans l'argumentation économico-éthique de notre époque, les notions de bonheur ou de satisfaction qu'on trouvait encore dans les philosophies classiques.?

Or, dans la mesure où la finalité d'une éthique est une quantité, il n'y a pratiquement plus de fin. On passe en effet de la logique d'un but final, aboutissement du devenir de l'être - Le Bien, le Beau, le Bonheur, toujours avec majuscule quand il s'agit de la fin ultime de la vie humaine - à une logique quantitative du «toujours plus», exprimée presque exclusivement en termes monétaires. On parle donc de niveau de vie, de revenu par tête d'habitants, de PIB, de taux de consommation d'énergie, de capital disponible, d'investissement par habitant, de rentabilité de l'investissement, etc., mais jamais du bonheur des hommes ou de leur épanouissement moral. Celui-ci est supposé, certes, puisque tout le système a pour but de satisfaire les besoins des acteurs de l'économie, mais comme leur bonheur n'est pas mesurable à l'aune de la quantité, il est pratiquement ignoré. Le jugement sur l'action spécifique de l'éthique de la prospérité s'exprime toujours en quantités et en chiffres. Or il n'y a pas de limites à l'addition des chiffres; il est toujours possible d'imaginer quelque chose de plus. C'est pourquoi la richesse, prise comme finalité, ne peut logiquement constituer une fin ultime de l'homme.

On le constate évidemment dans le vocabulaire lui-même. L'argumentation proposée par les sociétés avancées pour juger des questions de richesse, de

7 Galbraith souligne ce phénomène en termes d'économiste de la façon suivante: «The economist does not enter into the dubious moral arguments about the importance or virtue of the wants to be satisfied. He doesn't pretend to compare mental states of the same or different people at different times and to suggest that one is less urgent than another. The desire is there. That for him is sufficient. He sets about in a workmanlike way to satisfy desire, and accordingly he sets the proper store by the production that does. Like woman's his work is never done». (op. cit. p. 136) 
redistribution des richesses et de matières connexes qui concernent la vie humaine, s'édifie toujours à partir de minima, et jamais à partir de sommets accessibles et définitifs. Il faut un minimum de niveau de vie pour garantir les besoins humains fondamentaux; il existe des seuils de pauvreté, variables d'ailleurs d'un pays ou d'une région à l'autre, en dessous desquels la vie n'est plus considérée comme normale et devient inacceptable. Mais il n'existe pas de définition objective de la richesse, comme si celle-ci n'était pas une fin socialement accessible. Or cette inaccessibilité vient justement de sa nature quantitative. L'idée d'avoir assez semble contraire à la nature humaine. Dès qu'il se met à compter, l'homme demande davantage. Les valeurs semblent s'enchaîner mutuellement dans une sorte de spirale envoutante de besoins et de satisfactions, qui entraînent progressivement les sociétés contemporaines dans un engrenage qui peut, à la limite, devenir destructeur.

\section{L'équivoque permutation des fins et des moyens dans l'éthique du travail}

Il existait, dans les sociétés traditionnelles pré-capitalistes, une éthique du travail qui connaissait ses propres limites; celles-ci se trouvaient justement dans les buts visés. Quand l'homme constatait que ses efforts avaient abouti à la satisfaction de ses besoins fondamentaux, il pouvait y mettre fin. Le travail avait un but qu'il était possible d'identifier. L'image du paysan qui se repose, au moment où ses récoltes sont engrangées en suffisance, en est l'illustration la plus évidente. Pourquoi, en effet, poursuivre vainement son effort si la quantité utilisable de nourriture et d'énergie a été produite et accumulée pour la consommation directe des membres de la famille ou du groupe social concerné, et ceci pendant une période maîtrisable. Cette éthique du travail connaît une motivation fondée sur l'existence d'un besoin; elle accepte le repos quand celui-ci est satisfait. La notion du "suffisant» représentait une finalité justifiant l'abandon de l'effort.

Mais la rationalité de l'économie contemporaine ne fonctionne pas de cette façon. Comme le dit André Gorz: «la catégorie du suffisant n'est pas une catégorie économique: c'est une catégorie culturelle ou existentielle.» ... «si on commence par mesurer la richesse en numéraire, assez n'existe pas. Quelle que soit la somme, elle pourrait toujours être plus grande. La comptabilité connaît les catégories du «plus» et du «moins», elle ne connaît pas celle du "suffisant». ${ }^{8}$

Dès lors, l'économie ne travaille plus seulement à la satisfaction des besoins, mais à l'augmentation de ses moyens. Une société prospère est celle qui a atteint un niveau de production dépassant le stade de la seule

8 André Gorz, Métamorphoses du travail, Quête du sens, p. 142 
satisfaction des besoins. Il n'y a plus de finalité directement perçue par l'individu qui puisse justifier l'existence du travail. Or, quand une activité semble avoir atteint la fin qu'elle visait, et qu'elle tient à se maintenir en tant que telle, elle doit être justifiée par d'autres raisons que la fin poursuivie. Ce qui se produit alors est un glissement de la logique. L'activité, à l'origine instrumentale et fonctionnelle, devient elle-même sa propre finalité.

C'est ce qui se passe dans l'éthique de la prospérité. La valeur morale traditionnelle du travail est renversée. Il ne s'agit plus de travailler en vue d'une fin - combattre la disette par exemple - mais il s'agit de faire fonctionner l'économie pour que l'homme puisse travailler. C'est l'action qui est devenue le but du processus, et comme l'action humaine n'est évaluée dans ce système que par le seul profit qu'elle engendre, - comme nous l'avons dit, le travail lui-même n'est pas cumulable - c'est le profit qui devient à la fois la motivation, la fin et les moyens de l'action humaine. Il ne s'agit plus d'être heureux, résulat d'une action spécifique, il s'agit d'être prospère dans le travail, et de continuer à produire pour disposer de plus en plus de moyens de travailler, qui engendrent des moyens permettant de travailler davantage, etc, ... dans un enchaînement incessant d'activités visant l'accumulation du superflu. André Gorz l'exprime de la façon suivante:

«La passion rationalisatrice s'autonomisait vis-à-vis de tout but déterminé. A la place de la certitude vécue que «assez», c'est bien, elle faisait surgir une mesure objective de l'efficacité de l'effort et de sa réussite: le montant du gain. La réussite n'était donc plus une question d'appréciation personnelle et de "qualité de la vie», elle était mesurable par la quantité d'argent gagné, par la fortune accumulée. ... L'efficacité était mesurable et, à travers elle, la capacité d'un individu, sa vertu: plus vaut plus que moins, celui qui réussit à gagner plus vaut mieux que celui qui gagne moins» ${ }^{9}$

Dans une telle perspective, la société est entraînée dans une course effrénée à la croissance de la richesse, parune logique absolument contraignante, qui a pour conséquence une augmentation incessante des moyens. Dès lors:

ce ne sont plus les besoins des hommes que l'économie doit satisfaire, mais ceux de l'économie elle-même en tant qu'action humaine spécifique.

Ce passage à une économie devenue un champ d'activités justifiant sa propre existence par la simple augmentation de ses activités entraîne un

9 Ibid., p. 143 
changement radical des comportements humains et de leur signification. Nous voyons dans l'éthique de la prospérité la justification morale de ce glissement.

L'idée d'un "devoir» de travailler était justifiée par la nécessité de satisfaire aux besoins de la vie. "Produire son pain à la sueur de son front», comme le soulignait la condamnation de la Genèse. C'était le but de l'activité économique. Au moment où l'économie d'abondance permet de dépasser ce stade, elle est conduite à rechercher une finalité nouvelle. Elle trouve celle-ci, à première vue, dans la croissance de la richesse. Mais comme il n'est pas possible de mettre une limite à cette finalité, puisque la richesse n'a ni seuil ni plafond, il ne peut y avoir de fin dans l'objet de l'action. Ne reste donc plus que l'action elle-même comme propre finalité. C'est pourquoil'éthique qui s'en dégage logiquement, toujours à la recheche d'une finalité qui lui donne un sens, propose l'action humaine, c'est-à-dire le travail, comme nouvelle finalité de l'activité économique. Produire pour produire, c'est-à-dire augmenter sans cesse l'activité, devient ainsi la vertu suprême. L'action qui produit la richesse devient en elle-même la finalité de l'économie; la richesse n'ayant finalement pas d'autre but que de favoriser l'action, et à la limite d'en mesurer la valeur.

\section{Renversement des valeurs de l'action humaine}

Nous voyons donc naître une éthique dans laquelle les moyens sont devenus les fins. Le profit n'a pas d'autre raison d'être que le profit, la richesse que de faire naître la richesse, et ensemble de permettre aux êtres humains d'être les acteurs de ce processus. Or, toute action humaine n'est valable aux yeux de l'agent que si elle fait l'objet d'un jugement et est évaluée en fonction des valeurs de l'éthique dominante. Les critères qui permettent de juger les actions dans l'éthique de la prospérité ne peuvent être fondés sur la fin, qui n'en est pas une, ni même sur une adéquation des moyens à la fin poursuivie puisque celle-ci est infinie. Même celui qui se serait tué au travail, qui aurait produit jusqu'à la mort - et cette expression montre bien que l'action n'est jamais cumulative - ne pourrait être certain d'avoir atteint les limites de la richesse.

Il faut donc, pour éviter cette impasse et être capable d'évaluer l'action humaine, se concentrer exclusivement sur les moyens et non plus sur les fins. Or il n'est possible de juger des moyens sans tenir compte de leur finalité qu'en comparant leur valeur relative à d'autres moyens de même nature. Or ces autres moyens sont justement ceux d'autres personnes, d'autres agents de l'économie. Le critère du jugement, dans l'éthique de la prospérité, n'est donc pas fondé sur une norme idéale ou un absolu, mais sur une simple comparaison, également quantitative, avec d'autres manières de créer de la richesse. 
Dans la confusion créée par la permutation des buts et des moyens, qui caractérise les jugements de valeur portés sur l'activité économique au niveau global de la société, on on ne peut plus porter de jugement sur l'efficacité, car ce serait encore considèrer l'action en vue de ses fins; aussi l'on se contente le plus souvent d'un jugement d'efficience, qui considère l'action en elle-même et tente de l'évaluer en tant que telle, de façon quasi immanente. Ainsi l'on pourra dire que l'économie parfaite est celle qui fonctionne de la façon la plus efficiente possible, quels qu'en soient les buts!

Le jugement sur l'efficience, ou la performance - un concept qui permet de qualifier la «rectitude» de l'action, ou sa vertu, dans la perspective de l'éthique de la prospérité - ne peut être construit que sur une comparaison avec l'action d'autres agents, sur une échelle quantitative qui ne peut être que conventionnelle puisqu'elle $n$ 'a pas de fin. L'important, finalement, n'est pas d'être riche, c'est de savoir si l'on est plus ou moins riche que l'autre; ce n'est pas d'être efficace pour produire la richesse, mais de savoir si on le fait avec plus ou moins «d'efficience» que l'autre.

C'est ainsi qu'il existe des pays riches parce qu'il y a des pays moins riches, ou pauvres, et l'on peut reconnaître les pays pauvres ou les pays moins riches parce qu'il y a des pays plus riches; mais comment savoir si les uns ou les autres sont riches ou pauvres en soi? On ne compare d'ailleurs pas leur état, mais leur situation relative dans le temps, par rapport à un développement. Les sociétés trouvent leur valeur dans des courbes et des comparaisons beaucoup plus que dans leur réalité individuelle. En d'autres mots, les groupes humains et l'humanité en général sont répartis sur une échelle variable dans le temps qui est celle des degrés d'avancement vers l'enrichissement relatif du monde.

Les concepts utilisés dans l'analyse de l'économie de marché, qui ont avant tout pour but d'indiquer comment un système fonctionne et non pas quel but il poursuit, prennent dès lors des connotations morales valorisantes. Par ce biais, ce que nous avons appelé au premier chapitre le «moralisme économique», pénètre tous les jugements sur la société en offrant ses concepts comme critères de jugement de valeur. Ce sont de nouvelles valeurs favorisant le dynamisme de la comparaison qui viennent s'insérer dans le registre conceptuel de l'éthique de la prospérité. La notion de «concurrence», - fondement de l'universelle compétitivité de l'économie actuelle, - est présentée comme une nécessité de l'action, presque comme une vertu, dotée d'une certaine forme d'obligation dont le but est de stimuler l'activité. Or il est difficile de faire de la compétitivité une vertu en soi, puisque son existence ne dépend que de l'impossibilité de concevoir une finalité qui soit satisfaisante. A-t-on déjà vu, dans l'histoire de l'humanité, des sagesses mises en concurrence les unes par rapport aux autres? Devant l'absence de fin, l'être humain a perdu ses points de repère et ne peut donc se retrouver lui-même que par rapport à d'autres. Or, comme les éléments de comparaison sont exclusivement quantitatifs, qu'il n'y a à comparer que des «plus» ou des «moins», et comme l'on sait à l'évidence que la valeur 
«plus» et préférable à la valeur «moins», la compétition stimule l'action. L'important n'est pas tellement de savoir ce que l'on fait, mais comment son action permet de se situer sur l'échelle de l'enrichissement. La concurrence est donc bonne puisque qu'elle stimule l'action qui est elle-même devenue une fin en soi.

En fait, dans l'éthique de la prospérité, il n'y a pas d'actions bonnes et d'actions mauvaises; il y des actions plus efficientes, et d'autres qui le sont moins. Sont certainement mauvaises les attitudes et les conditions qui mettent en question cette concurrence. Certains en sont arrivés à croire que c'était la compétitivité entre les économies du monde qui était elle-même la source de la richesse.

Dès lors, l'attention se reporte sur la façon dont l'économie fonctionne plutôt que sur ce qu'elle produit. Les sociétés ne sont donc plus comparées selon leur revenu et leur bien-être, mais selon leur productivité. Bien produire, produire beaucoup, et si possible plus que son concurrent et à un meilleur coût, devient ainsi la caractéristique préférée et respectée de l'action humaine. L'idée de productivité, fondée sur les aspects quantitatifs et méthodologiques du travail, développée pour évaluer le coût réel de la production industrielle, devient une valeur en soi, attisée par l'universelle compétitivité des économies.

Finalement, l'idée de marché devient, logiquement, celle qui devrait englober toutes ces notions. Le marchéest le milieu favorable à l'épanouissement de l'éthique de la prospérité, car il est le lieu idéal où peut se manifester la nature propre de l'action éthique: la compétitivité. Le marché est donc un élément constitutif de l'éthique. C'est en lui que doit, pour ainsi dire, s'incarner la liberté. Il doit être respecté, ses règles doivent être précises, certes, mais suffisamment souples pour ne pas mettre en question le principe de la concurrence. On parlera donc de sa flexibilité et de son ouverture. D'autre part, plus un marché est grand, plus il facilite l'augmentation des activités compétitives garantissant par là la valeur éthique, c'est-à-dire l'efficience des agents qui trouvent leur finalité dans leur propre activité et non dans ce qu'ils produisent. Galbraith a donc pu déclarer, avec un certain cynisme prémonitoire, qu'on pourrait imaginer un système ou l'on passerait directement de la production à la poubelle, en se débarrassant ainsi de l'inutile étape de la consommation, celle qui concerne la satisfaction des besoins humains.

\section{L'homme pris au piège d'une éthique immanente}

Que devient l'homme dans une telle vision de la vie et de l'action? Son importance, dans un tel tourbillon d'activités, ne vient pas de son être, de son existence propre et de ses finalités individuelles, mais seulement de l'efficacité de son action, éthiquement jugée en fonction de sa contribution 
au processus de la prospérité. L'homme est devenu un moyen utilisé pour favoriser le fonctionnement du système collectif que l'éthique justifie, valorise et impose. Cette conception se manifeste, par exemple, dans l'utilisation de la nouvelle expression: «ressource humaine», pour désigner la participation de l'être humain au processus décrit ci-dessus. Or, les ressources d'une action n'en sont jamais la fin.

Certes, tout agent est en partie lui-même le moyen de la finalité qu'il poursuit. On lui propose donc, dans l'éthique de la prospérié, d'inscrire la richesse personnelle comme visée individuelle légitime. Les buts de l'individu sont alors en harmonie avec l'impulsion collective. Mais on sait qu'il est illusoire de faire croire à l'homme qu'il va ainsi pouvoir jouir, un jour, de sa fin ultime, puisque celle-ci est inaccessible, quantitativement sans limites. La richesse ne peut pas être le but ultime de l'homme. Or, comme nous I'avons montré plus haut, le sujet doit se retrouver lui-même dans la moralité qu'il construit, sinon il n'y a aucun progrès de la personne. Comment peutil donc se retrouver dans une simple quantité?

L'idée de «ressource humaine» peut être un piège, en particulier quand on l'utilise pour parler de la main-d'oeuvre dans le but de valoriser la contribution humaine à la poursuite générale de la richesse. Cette notion est liée également aux discussions sur la formation professionnelle. En d'autres termes, la formation ne serait qu'une amélioration ou une augmentation de la «richesse» de qualification des êtres humains en leur donnant une valeur ajoutée qui leur accorde les moyens d'une performance accrue. L'homme est donc bien un objet, dont on estime la valeur en fonction de sa situation sur l'échelle des qualifications. Car ces dernières ne sont considérées que comme des moyens individuels à mettre au service de l'accroissement de la richesse. Se former devient donc une forme de contribution supplémentaire à l'augmentation de l'efficience générale. Mais est-il possible d'imaginer une limite à cette croissance de la valeur humaine comme ressource de production?

Certaines interprétations de la crise du chômage illustrent également cette transformation de l'homme en objet du système de production. Alors que la récente crise du chômage a d'abord été considérée comme une tragédie personnelle pour les millions d'individus sans-travail qu'il a fallu soutenir, de plus en plus nombreux sont ceux qui pensent que la véritable catastrophe de nos pays est de ne pouvoir mieux exploiter ces immenses réserves de ressources humaines qui, en raison de l'oisiveté forcée qu'elles subissent, ne contribuent pas à la croissance de la richesse. Le sentiment d'inutilité et de marginalisation des chômeurs est donc renforcé par la logique des valeurs de l'éthique de la prospérité, puisque les «non-travaillant» ne participent pas à la valeur suprême de la société: l'action productrice comme but en soi. L'important, en effet, est de participer au processus. L.'homme doit être un rouage qui fait fonctionner plus efficacement l'immense machine collective qu'est le grand marché économique du monde. 
En maintenant cette finalité illusoire comme but ultime de la morale économique, en la proposant comme finalité individuelle à chacun des membres du groupe social, on obtient ainsi plus facilement une participation active des individus à l'avènement de l'éthique générale. Notre civilisation, après avoir été dominée par la religion, puis par les armées et les guerres, est aujourd'hui conditionnée par les systèmes de production. Ce ne sont plus les prêtres et les soldats qui sont les «héros et les saints» de l'éthique, pour reprendre l'image de Bergson, mais les managers, les entrepreneurs et les financiers.

Au milieu de ce processus, l'homme se perd. Certes, ce n'est ni la richesse ni l'abondance qui sont critiquables en soi. Il n'y a aucune raison de penser que les pauvres soient moralement plus respectables que les riches (en dépit de ce qui est dit dans l'Evangile!). Mais quand nous voyons que la seule valeur humaine qui survit est finalement la contribution ou noncontribution au processus par lequel la société dans laquelle il vit est plus productive que la société voisine, quand nous entendons qu'il ne s'agit plus pour l'homme d'être bon, mais compétitif, qu'il n'est pas important d'être prospère, mais qu'il faut être plus riche que son voisin, et que la comparaison est devenue la seule mesure du bien et du mal, il est nécessaire de réagir.

Or la faiblesse inacceptable de cette éthique est sa nature essentiellement immanente. Ce n'est pas une éthique ouverte, mais close, contraignante, refermée sur elle-même. Elle est en fait en contradiction avec l'idée même de marché qui devrait suggérer un espace d'ouverture et de différences. Il est possible en effet de défendre l'idée qu'une éthique économique liée à l'idée de marché devrait favoriser l'épanouissement des initiatives personnelles, et donner à chacun sa chance ${ }^{10}$. Mais il faut alors éviter de faire du marché le seul instrument de la prospérite, sinon il n'est plus le lieu de la liberté, mais celui de l'efficience. L'idée libérale qui le défend est ellemême prise au piège, comme nous allons tenter de le voir plus loin.

Pour sortir l'éthique économique de ce piège de l'immanence, il faut que l'individu s'affirme comme être autonome, qu'il s'inscrive dans un autre champ d'existence. Or c'est par l'affirmation d'un ordre supérieur de l'éthique que la persone peut justement relativiser l'immanence des valeurs d'efficience et de progrès quantitatif. Le retour du sujet responsable n'a pas d'autre raison. Comment en effet la prospérité pourrait-elle favoriser le respect d'autrui, la générosité, le don de soi, l'égalité et la justice?

Le piège ultime de l'éthique immanente de la propérité est en effet de dévaloriser les authentiques valeurs humaines. Or, on ne mettra des limites

10 Voir par exemple Edward J.J.M. Kimann, Marktethiek, en particulier les arguments de l'auteur en faveur de la pluriformité des acteurs économiques et du besoin d'une sphère privée qui garantisse la liberté et la responsabilité morale ( zingevende). 
à cette tentation que par un réveil de la conscience et une affirmation nouvelle de l'être en soi, du sujet, fondement et fin de tout. Le renouvellement de l'idée d'obligation morale peut contribuer à ce réveil. Certes, nul ne met en doute que nos sociétés avancées sont arrivées à un stade du développement économique et à un niveau de vie capables de permettre une véritable libération de l'homme. Ce que nous devons mettre en doute, ce sont les critères utilisés pour évaluer ce processus, car ils ne permettent plus de reconnaître cette liberté. Car si l'homme, pour prouver sa liberté dans son action doit renoncer à son être, il se meurt.

Pourtant c'est de liberté dont on parle le plus à notre époque. L'idéologie dominante n'est-elle pas le libéralisme? N'est-elle pas une pensée économique qui veut remettre l'individu, le sujet économique actif, l'entrepreneur, au centre de l'activité humaine? Représente-t-elle l'idéologie du processus que nous venons de décrire ou permet-elle de le dépasser dans la ligne de ce que nous envisageons? Nous devons maintenant tenter de voir en quoi, et jusqu'où, la philosophie du libéralisme est engagée (ou compromise?) par rapport à cette éthique de la prospérité.

\section{Le libéralisme et l'utilitarisme}

L'éthique de la prospérité n'est pas élaborée en un système global formalisé, malgré la cohérence avec laquelle elle s'impose comme paradigme de l'action. Elle découle d'une certaine logique d'interprétation de l'action humaine, et d'un glissement des valeurs et des principes éthiques vers des critères exclusivement quantitatifs et utilitaires, entraînant une permutation pernicieuse des fins et des moyens. Le développement de cette éthique de la prospérité ne doit cependant pas être identifié à la renaissance, parfois aggressive, de la pensée libérale qui caractérise la réflexion politique et économique de cette dernière décennie. La coïncidence historique ne doit pas servir à établir une relation causale ou une cohérence idéologique. Le libéralisme se présente toutefois comme une doctrine éthique, et les positions qu'il défend aujourd' hui dans le débat sur le rôle de l'Etat dans l'économie et dans la croissance de la prospérité nous obligent à un rapprochement évident. Il n'est donc pas possible d'éviter la discussion de cette question. Le libéralisme est-il la source, ou du moins une des sources, de l'éthique de la prospérité?

Disons tout de suite qu'il n'y a pas qu'un seul libéralisme, une doctrine unique, suffisamment élaborée et complète, mais de multiples formes de pensée libérale, de multiples auteurs qui s'en inspirent, et sa pratique économique et politique concrète revêt de nos jours des formes très diversifiées. Cette multipicité des "pratiques» du libéralisme porte d'ailleurs souvent à confusion. Ne parle-t-on pas, en effet, de démocraties «libérales» pour qualifier tous les systèmes politiques «ouverts» qui ne sont pas spécifiquement 
dominés par un tyran, un dictateur ou une junte autoritaires? ${ }^{11}$ Or les nuances qui distinguent ces systèmes les uns des autres sont souvent immenses, et beaucoup d'entre eux pourraient tout aussi bien bien être qualifiés de "socialistes", "communautaires» ou même franchement «étatiques», autant de dénominations que les authentiques «libéraux» rejetteraient comme contraires à leurs principes. Il n'est donc pas possible de présenter ici une théorie simplifiée du libéralisme, car celle-ci serait forcément abusive.

Nous nous attacherons avant tout aux fondements éthiques du libéralisme, et nous en distinguons principalement trois, en nous inspirant du livre récent de Francisco Vergara: Introduction aux fondements philosophiques du libéralisme, qui en fait une synthèse très nuancée.

Il y a trois tendances du libéralisme, depuis le libéralisme classique né au XVIIIe siècle, jusqu'au renouveau contemporain que l'on peut appeler, "ultra-libéral», pour reprendre la définition de Vergara, ou «néo-libéral». Ces trois tendances se distinguent par les principes éthiques de base qu' elles prennent pour fondements.

Le libéralisme classique, tout d'abord, qui connaît deux tendances. Celles-ci se rattachent à des doctrines éthiques traditionnelles, à savoir: «la doctrine appelée aujourd'hui «utilitariste», selon laquelle la seule manière de juger si les lois et les institutions sont bonnes ou mauvaises est d' examiner leurs effets sur le bonheur des hommes, et la doctrine dite du «Droit naturel», selon laquelle le critère éthique qui permet de juger si les lois sont bonnes ou mauvaises est leur conformité avec l'ordre naturel » ${ }^{12}$. La première tendance identifie le bien avec le bonheur; la seconde voit dans la Justice le fondement souhaitable de toutes les lois. Les deux doctrines affirment que l'existence de la liberté est le meilleur moyen d'atteindre ces buts. L'utilitarisme reconnaît que la liberté des individus est le meilleur instrument pour obtenir le «plus grand bonheur du plus grand nombre»; l'autre école affirme que le principe de liberté est celui qui est le plus conforme au Droit naturel. On retrouve dans la première école des auteurs comme Hume, Bentham et Smith, et dans la seconde des auteurs continentaux et américains comme Turgot, Condorcet et Jefferson. A long terme, il est possible d'y voir déjà une préfiguration des conceptions différentes de l'économie que l'on retrouve aujourd'hui dans les différentes formes du capitalisme.

La troisième tendance, que Vergara appelle «ultra-libérale», est celle qui, commencée au XIXe siècle, a visé à limiter au maximum le rôle de l'Etat (Bastiat en France et Herbert Spencer au Royaume Uni) et a été renouvelée aujourd'hui avec des penseurs comme Friedrich Hayek et Milton Friedman. Cet «ultra-libéralisme» introduit un autre critère ultime de l'éthique, et en

\footnotetext{
${ }^{11}$ Francis Fukuyama, The End of History and the Last Man, parle par exemple d'une "révolution libérale mondiale»; cf. Chapitre 4, p. 39

12 Francisco Vergara, op. cit., p. 12
} 
fait le fondement de la doctrine, à savoir: la quantité totale de liberté. Milton Friedman écrit: «..en tant que libéraux, nous prenons la liberté de l'individu, ou peut-être de la famille, comme but ultime permettant de juger les institutions sociales» ${ }^{13}$.

Si l'ultra-libéralisme est celui qui semble le plus répandu aujourd'hui, c'est pourtant le libéralisme utilitariste qui nous intéresse le plus directement par rapport à ce que nous avons défini comme l'éthique de la prospérité. En effet, selon la doctrine utilitariste, la valeur morale d'une action dépend exclusivement de la quantité de plaisir qu'elle produit. Le problème est dès lors de savoir comment calculer cette quantité de plaisirs ou de bonheur que peut entraîner l'action. Jeremy Bentham a tenté d'élaborer une méthode pour y parvenir. Cette exigence découle du but à atteindre, qui n'est pas nécessairment le maximum de plaisirs pour l'individu, mais le "plus grand bonheur pour le plus grand nombre». Il est toutefois évident, et Bentham l'a déjà reconnu, qu'il n'est pas possible de faire une addition purement mathématique de choses qui sont difficilement quantifiables; le calcul hédoniste demeure un «idéal». Ce qui compte, c'est le principe qui demande d'évaluer chaque action, dans chaque circonstance particulière, en fonction du maximum de bonheur qu'elle peut apporter pour le plus grand nombre. Chaque individu est ainsi appelé à juger son action par un principe d'universalisation de son utilité. C'est ce principe qui doit demeurer la source d'inspiration de tous les jugements moraux ou de toutes les décisions législatives.

C'est dès l'époque d'Adam Smith que l'idée de substituer la notion de consommation à celle de bonheur a été progressivement introduite dans la pensée économique pour mesurer l'efficacité d'un système ${ }^{14}$. Tout en reconnaissant qu'un calcul quantitatif devenait plus facilement faisable, les difficultés n'ont jamais été totalement vaincues dans la définition de ce qu'est l'utilité économique globale ${ }^{15}$. Mais la consommation peut être plus

13 cité in Ibid., p. 115

14 Adam Smith n'est peut-être pas lui-même à l'origine de ce glissement, ill' a cependant favorisé en faisant de la consommation la finalité première de l'activité économique: «La consommation est l'unique but, l'ultime terme de toute production, et l'on ne devrait jamais s'occuper de l'intérêt du producteur, qu'en tant qu'il le faut seulement pour favoriser l'intérêt du consommateur. - Cette maxime est si évidente par ellemême, qu'il y aurait de l'absurdité à vouloir la démontrer». Il ajoute même, ce qui n'est pas sans importance dans la perspective de notre débat: «Dans le système que je combats, l'intérêt du consommateur est à peu près constamment sacrifíe à celui du producteur, et ce système semble envisager la production et non la consommation, comme le seul but, comme le dernier terme de toute industrie et de tout commerce». La richesse des Nations, Livre IV, Chap. VIII, Volume II, p. 279

15 Voir à ce sujet les travaux de Pareto et son idée d'ophélimité, ou utilité économique, qui cependant ne peut être étendue à toute la société en raison de l'hétérogénéité des jugements subjectifs. 
facilement quantifiée que l'utilité dans l'évaluation des politiques. Il s'agit en effet de voir, dans toutes les mesures et réglementations qu'il faut prendre ou ne pas prendre, et finalement dans la libre action des agents économiques, ce qui constitue le meilleur moyen d'assurer et de développer la consommation. Celle-ci permet de remplacer une notion morale, comme le bonheur, qui n'est pas mesurable et qui ne peut être vérifiée que de façon subjective, par une notion quantifiable qui, elle, peut être collectivement vérifiée. En substituant la notion de consommation à celle de bonheur, la pensée propose une morale plus objectivement et plus rapidement mesurable dans ses effets, plus facilement maîtrisables aux yeux des législateurs, et plus pratique à manier pour convaincre les masses.

Cet artifice par lequel la justification morale n'est plus à rechercher dans une valeur subjective, même universalisée dans son principe par la recherche du bonheur du plus grand nombre, mais dans une donnée économique objective et quantifiable, représente un renversement fondamental du paradigme éthique par l'utilitarisme. En effet, non seulement la satisfaction humaine n'est plus à rechercher dans le plaisir «ressenti» subjectivement (théorie tout à fait cohérente avec l'empirisme de Hume et le rejet de la métaphysique par la philosophie anglaise) mais elle se trouve désormais en dehors de lui, dans le monde des quantités vérifiables, qui peuvent être saisies par les mathématiques et la méthode scientifique. En d'autres mots, la finalité de l'éthique et la recherche du bien se détachent progressivement de la satisfaction subjective de l'être humain, incarnée dans ses sensations et ses sentiments, pour s'attacher aux quantités matérielles que son action maîtrise. Le bonheur n'est plus dans l'être mais dans l'action, la métaphysique est définitivement écartée de la morale pour être remplacée par la mesure des biens. L'avoir quantifié se substitue à l'être.

On ne comprendra toute l'importance de ce renversement de la pensée morale qu'au moment où l'économie sera vraiment capable de fournir tous les biens que la consommation peut souhaiter, et même plus que le souhaitable. Il n'est en effet pas étranger au développement de la rationalité économique du capitalisme contemporain, dont nous avons souligné le manque de finalité objective plus haut. Vergara écrit très justement: «A l'époque d'Adam Smith, il était parfaitement raisonnable d'utiliser la consommation comme variable à maximiser à la place du bonheur; il était raisonnable de considérer que l'augmentation de la consommation coïncidait, en règle générale, avec l'augmentation du bonheur. Dans de nombreux pays du monde cette assimilation semble encore largement valable, mais on peut se demander si, dans les pays les plus développés, on peut encore raisonner de cette manière. C'est bien entendu là une question qui dépasse le sujet de ce livre.»16

16 Francesco Vergara, op. cit., p. 62 
Si telle n'est pas la question du livre de Vergara, c'est fondamentalement celle que nous devons soulever dans la perspective de notre interrogation. L'idée de bonheur trouve un développement très particulier, sous l'influence de l'empirisme anglais. D'abord, l'utilitarisme qui se développe à partir de ces réflexions sur la moralité, conduit à deux approches distinctes de l'économie; plus individualiste dans les îles anglo-saxonnes et aux EtasUnis d'une part, et plus juridique et dans une certaine mesure plus sociale sur le continent européen de l'autre. Nous reviendrons sur cette différence plus loin. Mais dans aucun de ces deux cas, pris théoriquement, nous retrouvons la permutation des fins et des moyens que nous avons constatée dans le contenu de l'éthique de la prospérité. La poursuite du bonheur, même du bonheur matériel mesuré à l'aune de la consommation, demeure une finalité, certes utilitaire, qui a toujours puêtre considérée comme satisfaisante par rapport aux besoins humains. Ce n'est que sous d'autres formes que le néo-libéralisme contemporain a pu conduire à l'éthique de la prospérité, telle que décrite ci-dessus, quand les finalités de l'activité économique sont devenues prépondérantes et ont dominé les finalités sociales.

Il a fallu pour cela que l'économie, par l'industrialisation, permette de dépasser en quantité les besoins de consommation, devienne capable de produire plus qu'il ne fallait pour faire le bonheur des hommes, et favorise ainsi l' accumulation du profit, donnant naissance au capitalisme contemporain. C'est ainsi que l'on est passé de la consommation comme mesure du bonheur à la croissance de la richesse comme finalité de l'action, que nous retrouvons comme fondement de l'éthique qui caractérise le capitalisme contemporain. Aujourd'hui, comme nous l'avons souligné plus haut, ce n'est plus la consommation qui est la manifestation de la satisfaction économique des sociétés, c'est la production.

Ce n'est donc pas en tant que tel que le libéralisme a pu donner naissance à l'éthique de la prospérité. Par contre, en «matérialisant», pour ainsi dire, la mesure du bonheur humain par l'idée de consommation, il a contribué à faire glisser la recherche de la finalité dans la satisfaction des besoins à l'augmentation des moyens. L'éthique de la prospérité n'est pas une éthique typiquement libérale, nous dirons qu'elle est avant tout une éthique du capitalisme, tel qu'il s'est développé au cours de ces deux derniers siècles à partir du libéralisme d'Adam Smith. Quand la performance et la productivité du système de production dépassent les besoins de la consommation, le système n'a d'autre but que son propre développement, et substitue l'acquisition et la croissance des moyens à sa finalité première. Le capitalisme contemporain, en particulier celui qui est pratiqué par le néo-libéralisme, peut être caractérisé de cette façon. Mais comme pour le libéralisme, il y a plusieurs formes de capitalisme, et il s'agit de voir maintenant en quoi ces formes diffèrent. 


\section{Le capitalisme, le néo-libéralisme et l'éthique sociale}

Il est évident que le capitalisme et l'économie de marché, qui survivent aujourd'hui à la disparition du communisme, représentent les composantes essentielles du paradigme socio-économique sur lequel sera fondée l'éthique de la prospérité. Mais dans la diversité des cultures qui compose la carte du monde, il semble que l'on puisse distinguer deux modèles de ce capitalisme, qui vont tenter de conquérir leur droit à incarner la vérité de la nouvelle éthique. Nous reprenons ici la thèse du récent livre de Michel Albert, Capitalisme contre Capitalisme, et essayons de voir dans quelle mesure elle nous met sur le chemin d'une solution au problème éthique que nous avons soulevé.

Michel Albert distingue deux formes de capitalisme, l'une qu'il appelle «néo-américaine», développée sur les traditions morales de ce pays mais renouvelée et dynamisée dans les années 1980 par la politique économique néo-libérale du président Reagan, et l'autre qu'il appelle «rhénane», ou «européenne» au sens continental du terme, qui trouve sa source dans l'idéologie allemande de la Sozial Marktwirtschaft. La première forme se caractérise par la réussite individuelle et le profit financier à court terme, l'autre par la valorisation de la réussite collective, le consensus et le souci du long terme. Entre les deux, mais penchant plus évidemment du côté de la forme néo-américaine, il situe le capitalisme de l'Angleterre de Mme Thatcher, qui tout en prônant le retrait de l'Etat de la vie économique, n'a pas osé - ou réussi - à détruire entièrement le système de sécurité sociale et de santé que l'Angleterre a instauré chez elle depuis la deuxième guerre mondiale, sur la base des idées que Lord Beveridge avait développées en s'inspirant du modèle de Bismarck.

Notre but n'est pas ici d'analyser dans le détail l'ensemble de l'ouvrage de Michel Albert, dont la dimension éthique est pourtant évidente, car ce livre tient davantage de l'analyse économique et se concentre davantage sur le rôle et le fonctionnement des institutions clés d'une société capitaliste, que sur la réflexion éthique qui fait l'objet de notre propre intérêt. Mais la distinction établie par Michel Albert a le grand avantage de faire mieux apparaître les limites éthiques d'une certaine forme de capitalisme. Certes, les deux capitalismes présentés ici peuvent se référer à l'éthique de la prospérité, car les deux visent, chacun à sa manière, l'enrichissement de la société et des individus. Mais leur différence est essentielle, à nos yeux, dans la détermination du rôle éthique respectif de l'individu et du groupe, sans lequel aucune morale sociale ne peut être construite.

Exprimée dans des termes plus proches de nos préoccupations, nous dirons que la forme néo-libérale, ou «néo-américaine» du capitalisme préconise avant tout la prospérité individuelle et considère la société comme instrumentale pour atteindre ce but. Ce dernier doit être à très court terme, car la vie individuelle est courte, et comme chacun doit recommencer sa 
propre poursuite du bonheur, ce n'est pas dans les structures sociales durables qu'il faut investir la garantie de son bonheur. La société ne doit donc pas intervenir directement mais, en se tenant à distance, elle doit garantir que la poursuite de la richesse par chacun, sous la bienveillante intervention de la «main invisible», contribue au bonheur de tous. Il faut le moins d'intervention possible de l'Etat, et surtout ne pas espérer un enrichissement de la société en tant que telle, même si cet enrichissement peut être acquis au bénéfice des générations futures. Le droit à la "poursuite du bonheur», exprimé dans la déclaration d'indépendance des Etats-Unis, est un droit individuel qui n'implique pas le devoir d'assurer le bonheur des générations futures. C'est donc indirectement, par la nature des lois du marché et de l'échange, que l'enrichissement de chacun peut être utile à la prospérité de tous.

La forme rhénane ou européenne du capitalisme, au contraire, pense qu'en préconisant la prospérité sociale il est possible d'assurer la prospérité du plus grand nombre, et qu'en travaillant pour la société ou l'Etat comme pour lui-même, l'individu n'oppose nullement sa richesse à la richesse des autres. L'enrichissement de tous peut donc être poursuivi au bénéfice de la prospérité de chacun. Le bonheur est personnel, certes, mais peut faire l'objet d'une poursuite «solidaire», ce qui donne au groupe une responsabilité envers la prospérité de chacun de ses membres. Certes il faut la liberté d'entreprendre, mais l'Etat doit garantir que le bonheur poursuivi est un bonheur à long terme et qu'il peut être atteint par le développement social. La société n'est pas seulement instrumentale dans l'accroissement de la prospérité individuelle, mais partenaire dans une croissance commune à long terme.

Michel Albert illustre ces deux tendances par l'exemple des assurances d'une part, et de la propriété des entreprises de l'autre. Les assurances sont, dans le cas de type «alpin» ou continental, fondées sur la solidarité de tous, avec un effort mutuel et égal de tous les participants, le bénéfice se partageant en fonction des besoins des victimes; dans l'autre cas, de type «maritime» ou anglo-saxon, l'effort et le bénéfice sont variables selon les cas individuels, et déterminés par contrats individuels selon la valeur marchande de l'objet à assurer. Quant à l'entreprise, elle est considérée dans le capitalisme européen comme une organisation qui doit faire du profit par le travail de ses membres, à laquelle participent tous ceux qui y travaillent, les détenteurs du capital comme les travailleurs. Dans le capitalisme néoaméricain, l'entreprise est une simple marchandise que l'on achète, que l'on vend, qu'on développe ou qu'on liquide en fonction du bénéfice qu'elle peut engendrer.

Les différences essentielles entre ces deux approches sont les suivantes. Le néo-capitalisme américain développe une éthique où l'argent est roi et autorise tout dans la mesure où le profit engendre le profit à court terme. L'efficacité de l'action, c'est-à-dire sa "rectitude morale» du point de vue de l'éthique de la prospérité, doit être rapidement visible, mesurable et 
contribuer à une accumulation de richesse directement quantifiable et individualisée. Dans l'éthique du capitalisme «rhénan», également tournée vers la prospérité, le profit doit être progressif, s'inscrire dans un développement à long terme et contribuer à un mode de vie, de nature sociale, qui peut s'épanouir en dehors de la vie économique elle-même: loisirs, pensions, etc. Dans le premier cas, c'est autour de l'activité individuelle que l'efficacité se mesure; dans le second cas c'est par rapport aux résultats sociaux. Dans le premier cas il faut tenir l'Etat à distance, car il risque d'être un obstacle à la croissance de la richesse individuelle; dans le second il «doit» intervenir pour garantir un développement auquel tous ont le droit de participer. Nous pourrions dire, en d'autres mots, que les éthiques de ces deux capitalismes s'opposent, le profit de l'un se dressant contre le développement préconisé par l'autre.

Les simplifications et les dichotomies ont l'avantage de clarifier les idées et les conceptions, mais elles ont la faiblesse de voiler les nuances de la réalité. Il serait faux de conclure, à partir de cette brève analyse, que l'éthique de ces deux capitalismes diffère totalement. Certes il y a une morale économique américaine ou anglo-saxone qui se distingue de la morale économique européenne continentale, mais cette différence est davantage dans l'équilibre des facteurs qui l'expliquent que dans les principes qui la fondent. Ces deux types d'éthique sont du même ordre, ils visent tous les deux l'augmentation de la richesse individuelle et sociale, - et en ce sens le livre de Michel Albert s'inscrit dans le courant général de l'éthique de la prospérité - mais ils accordent peut-être une importance différente à l'individu dans le partage des responsabilités morales de l'économie. Or c'est la place de l'individu en tant que sujet qui est essentielle pour qu'il ne soit pas dépouillé de toute responsabilité par les exigences de l'éthique de la prospérité. C'est cette question qu'il nous faut maintenant aborder.

\section{Le sujet responsable}

Une éthique de la productivité et du succès, qui ne peut déterminer avec précision les buts qu'elle poursuit, parce qu'elle est dans l'impossibilité de leur fixer une limite, a tendance à développer une force de conviction et de contrainte à sa propre image. Elle soutient dans ce but des valeurs capables de motiver les énergies individuelles dont elle a besoin pour s'actualiser, d'autant plus qu'elle ne peut pas, ce qui serait contraire au libéralisme qui l'inspire, faire appel à la coercition légitime de l'Etat. La contrainte sociale fait partie du phénomène moral et existe dans toutes les sociétés. Mais nous voulons d'abord souligner ici, pour mieux faire apparaître l'impasse dans laquelle conduit l'éthique de la prospérité, la force avec laquelle cette contrainte sociale qu'est la course à la richesse conditionne les individus, et comment elle les emprisonne dans un processus que plus personne ne 
semble maîtriser. Or nous avons vu qu'une éthique fondée sur la seule contrainte n'était pas réellement, c'est-à-dire formellement, morale.

La première contrainte est celle du travail et de la performance. Dans une course généralisée au progrès matériel, mesuré surtout en termes globaux et rarement en fonction du succès particulier des individus, la pression qu'exerce le sytème de production sur la performance individuelle est énorme. Les individus sont conditionnés à travailler beaucoup, à participer activement au processus et à produire toujours davantage. On attend des êtres humains qu'ils orientent toutes leurs capacités d'action et leurs valeurs vers le but général et commun de la richesse. L'action humaine individuelle s'inscrit ainsi entièrement dans la même course de la productivité et de la compétition. Les individus sont donc pris dans l'engrenage du processus social général, et deviennent de plus en plus instrumentaux dans l'activité économique. On attend d'eux également qu'ils développent leurs propres compétences individuelles, sous forme de qualifications supplémentaires, afin de contribuer à l'amélioration générale des ressources humaines. La contrainte sociale prend dès lors la forme d'une valorisation guidée de l'être humain par un système subtil de récompenses et de promotion dans le cadre des valeurs spécifiques de la société d'abondance. Le quantitatif intervient de ce fait directement dans l'évaluation morale de l'activité humaine, et une hiérarchie des valeurs s'établit, qui place le travail au sommet des valeurs d'accomplissent personnel et de générosité sociale. ${ }^{17}$

Peut-on en conclure que l'éthique de la prospérité serait tentée de favoriser la notion d'un «devoir au travail»? Le travail deviendrait-il ainsi une obligation? Contrairement à ce qui a existé dans notre tradition occidentale avant l'avènement des sociétés d'abondance, quand la pauvreté constituait le statut de la majortié de la population, l'obligation de travailler ne viendrait pas d'une condamnation, celle qui marque la condition humaine depuis la punition d'Adam et d'Eve, ou qui caractérise l'état des individus inférieurs, esclaves ou serfs, ${ }^{18}$ mais se présenterait au contraire, dans l'éthique de la prospérité, comme une valeur positive située très haut dans la hiérarchie des valeurs. Ceux qui travaillent, dans les sociétés d'abondance, se retrouvent au sommet de la pyramide des privilégiés. Les héros du travail sont avant tout efficaces et productifs, leurs résultats sont cités en exemple, ils deviennent une nouvelle élite sociale, celle qui contribue le plus au développement de la société prospère.

Le paradoxe de cette évolution est que, dans une société où la consommation est considérée comme l'instrument privilégié de la quête du bonheur

17 Voir à ce sujet Marcel Bolle de Bal, Les doubles jeux de la participation, Rémunération, performance et culture

18 Voir Les Sept piliers de la civilisation du Travail, de Gabriel Fragnière, René van Neuss et Chantale Delforge 
individuel, où les industries du loisir croissent dans des proportions inconnues jusqu'à ce jour, nous découvrons que la place du travail augmente, que celui-ci est de plus en plus valorisé, et que toutes sortes de moyens de pression et de récompenses matérielles sont imaginés pour inciter - dira-ton obliger? - les individus à travailler. On a constaté, par exemple, que le temps consacré au travail par les familles augmente depuis que les femmes participent activement au système de production ${ }^{19}$. Or, en dépit des difficultés personnelles ou familiales qu'il peut engendrer, ce travail n'est pas considéré comme un fardeau, mais comme une valorisation de l'individu. Il semble donc que le travail soit devenu une des principales valeurs morales dans l'éthique de la prospérité.

Toutefois cette valeur morale a des limites car sa finalité ultime ne se trouve pas dans l'agent, mais dans les résultats externes. N'oublions pas que le travail n'est pas valorisé en lui-même mais dans ce qu'il rapporte, et qu'il n'est pas lui-même sujet de croissance. Ce n'est pas la quantité de tavail supplémentaire qui est source du bonheur humain. Seule sa qualité par rapport à la vocation de la personne peut constituer un progrès. Mais il n'existe pas de mesure quantitative globale pour évaluer le nature du rapport subjectif de l'individu à son travail. La valorisation personnelle par le tavail demeure donc très relative dans l'éthique de la prospérité. La responsabilité rattachée à la valeur travail n'est liée qu'à l'efficience, à la performance, à l'utilité et au succès matériel, et non au développement de la personne. Le travail productif est respecté, et l'individu l'est également en tant que travailleur efficace, mais sa valeur propre, indépendamment de son travail, est souvent très secondaire. La productivité, de son côté, implique l'exclusion sociale des moins productifs, exclusion justifiée au nom des valeurs dominantes du travail, un peu de la même façon que, dans les sociétés aux moeurs très strictes, les déviants sont marginalisés. La position des individus rejetés par une société du travail leur enlève le droit de ressentir l'obligation de travailler. Il y a un rejet non seulement de l'activité, mais même de la moralité qui est liée à la productivité du travail. C'est la conséquence de la moralisation du travail par ses seuls résultats quantitatifs. Le travail n'est donc plus une valeur humaine, c'est un instrument en vue d'une fin.

Cette tendance contient en elle le danger de la déresponsabilisation générale des individus. Si le travail n'est pas lié à une obligation de l'être, en tant qu'être humain, mais seulement à un calcul de rentabilité et de productivité, seule une pression sociale artificiellement entretenue sera capable de motiver les travailleurs et de maintenir leur motivation. C'est le rôle premier de la multiplication récente des éthiques d'entreprises utilisées

19 "Une famille danoise moyenne travaille 70 heures par semaine, contre 48 heures juste après la fin de la seconde guerre mondiale», Per Sorensen dans: Europe 93, Entreprises et familles, quelles stratégies de rencontre? 
comme instruments de motivation. Ceci peut, à long terme, entrainer une dévalorisation de l'individu au profit de ses seuls résultats, avec pour conséquence une désintégration de la valeur de l'être individuel dans la richesse qu'il produit. Il en est de même du consommateur dont la société manipule les goûts et hypertrophie autant que possible les moyens de consommation afin que la satisfaction de ses besoins contribue utilement, et efficacement, à la progression de l'instrument de production.

Ce n'est pas la possession de la richesse qui déshumanise la société, c'est l'éthique construite autour de la production et de l'utilisation de cette richesse qui transforme l'individu en un simple objet fonctionnel. Voilà pourquoi nous croyons nécessaire de redonner au sujet la place centrale dans la construction de sa propre éthique. Une réflexion devrait être conduite en particulier autour de la notion de «travail vocation», mission personnelle ou accomplissement de soi, que l'on retrouve par exemple dans la tradition luthérienne, et dont nous avons retrouvé l'orientation dans les conceptions éthiques de Max Scheler ${ }^{20}$.

Si l'homme s'abandonne trop aux pressions extérieures, même à celles qui semblent le satisfaire (ne jouit-il pas d'une consommation abondante?) mais qui n'ont pour but que de mieux l'exploiter, il sera peu à peu conduit soit à renoncer à être un individu, sujet de sa propre action, soit à rejeter violemment les valeurs du groupe dans lequel il se développe. Le risque inhérent aux sociêtés entièrement dominées par l'éthique de la prospérité est donc de faire naître une réaction de rejet de tout le corps social, le jour où l'individu n'est plus capable de supporter davantage le joug des obligations qui le lient à la production de biens matériels dont la seule mesure quantitative demeure en permanence extérieure à lui-même. Or ce rejet de la société est pour le sujet une forme de suicide. A la limite, l'éthique de la prospérité déresponsabilise l'homme vis-à-vis de lui-même.

C'est pour lutter contre ce danger que nous proposons de revenir à l'obligation morale comme fondement de l'éthique afin de reconstituer l'homme comme sujet responsable. Quand l'être humain est entraîné dans une action dont il ne maîtrise ni la forme ni la fin, il doit tenter de retrouver sa propre identité et ses propres ressources. Or c'est dans ce qui l'oblige, parce que sa liberté est alors respectée, qu'il peut découvrir ce qu'il est réellement. Face au succès d'une société omnipotente, il faut faire à nouveau appel au sujet, à sa responsabilité, à sa créativité afin de redonner à l'activité humaine, une fin digne de l'agent qui l'accomplit. C'est le sens même du dernier livre de Alain Touraine, Critique de la modernité, qui parle du «retour du sujet» au moment où les empires étatiques collectivistes s'écroulent et où sont mises en question les sociétés mercantiles: «le retour du sujet

20 Voir également Les Sept piliers de la civilisation du travail: la troisième valeur du travail. 
marque le déclin de tous les principes unificateurs de la vie sociale, que ce soit l'Etat ou le marché $\otimes^{21}$. «Le respect du sujet est aujourd' hui la définition du bien: qu'aucun individu ou groupe ne soit considéré comme un instrument au service de la puissance ou du plaisir ${ }^{22}$. «Le mal est la domination de l'homme sur l'homme en sa transformation en un objet ou en son équivalent monétaire $\aleph^{23}$.

Mais en enracinant l'idée de sujet dans le rapport qu'il entretient avec sa propre obligation, nous suggérons plusieurs idées. D'abord nous opposons la notion de sujet à celle d'individu, c'est-à-dire à l'idée d'une société composée uniquement de monades fermées sur elles-mêmes qui seraient dotées de valeur éthique par le simple fait d'exister contre le groupe. Ce n'est pas en affirmant l'individualisme de chacun que l'on mettra en échec la toute puissance de la contrainte sociale. Au contraire, l'individualisme n'a jamais mis en danger que la vie sociale, et non pas les déviations de celleci. Le but de notre effort n'est pas de contrarier la société dans son existence, c'est d'empêcher qu'elle ne nie l'homme dans sa nature d'être responsable. L'individualisme est un danger pour la société, donc pour l'homme dans sa nature sociale. L'homme sujet, au contraire, s'affirme dans sa relation avec les autres, non en la niant.

Deuxièmement, l'idée de sujet et celle d'obligation sont corollaires, comme nous l'avons largement développé dans notre analyse des deux dimensions de la morale. Ceci implique qu'un retour réflexif de la conscience sur ce qui oblige l'homme au plus profond de son intimité d'être subjectif n'a rien a voir avec la tentation d'établir un solipsisme moral qui ferait de l'individu isolé la mesure de toutes choses. Le sujet ne prend conscience de lui-même que parce que l'autre existe. Son affirmation est donc, par nature, profondément solidaire. Affirmer le sujet, en ce sens, c'est donc faire appel à un libéralisme total qui ne se contente pas de mesurer les succès quantitatifs de sa doctrine, mais qui accorde la primauté de l'être sur le faire, de la finalité sur l'efficience.

Ceci est particulièrement vrai dans la vie économique où l'initiative individuelle se situe le plus souventà l'origine de développements prometteurs. Il faut des sujets responsables capables de trouver en eux-mêmes le sens de leur propre obligation, et non pas des acteurs passifs soumis à des décalogues de lois et de règlements. Comme il n'y a pas de marché sans une réglementation de base qui permette le jeu des libres agents, ainsi il ne peut y avoir d'économie entièrement libre sans des agents maîtres d'eux-mêmes. Et il faut, dans un monde où les activités humaines peuvent se développer sans limites, des sujets capables de choisir de façon responsable les fins qu'ils vont poursuivre.

\footnotetext{
21 Alain Touraine, Critique de la modernité, p. 266

22 lbid., p. 268

23 Idem
} 
Enfin, affirmer le sujet comme être toujours responsable dans sa vie sociale, c'est introduire notre réflexion morale dans l'histoire et lui donner un sens. Nous allons découvrir cette dimension dans le chapitre suivant, mais nous devons souligner dès maintenant que le retour du sujet dans la préoccupation éthique est avant tout la reconnaissance d'une maîtrise de l'action humaine sur l'évolution de la vie sociale, et l'affirmation d'un devenir social fondé sur les valeurs, donc nécessairement libre et précaire. L'homme ne s'inscrit pas dans le mouvement de son histoire en acceptant passivement les tendances et la mode dominantes, celles qui sont de «son temps», - comme l'éthique de la prospérité semble l'exiger - mais en acceptant de participer à la construction de l'avenir par des décisions responsables. S'il peut y avoir un progrès éternellement recommencé de l'humanité, ce que nous discuterons dans le dernier chapitre de cet ouvrage, il dépend des actions de chacun, car la moralité est, comme le travail et la vocation personnelle, reconstruite avec chaque vie individuelle. Le retour du sujet signifie que le bien n'est pas le résultat de mon adaptation au temps présent, mais de ma contribution à mon avenir. 


\title{
Chapitre 8
}

\section{La construction de l'histoire et la justice}

\author{
C'est le choix de l'activité humaine \\ décisive qui détermine la conception \\ générale de l'histoire.
}

Raymond Aron

Introduction à la philosophie de

l'histoire

La seule obligation que $\mathrm{j}$ 'ai le droit d'assumer, est de faire à chaque instant ce que je considère comme juste.

Henri David Thoreau La désobéissance civique

Si l'obligation envers autrui conduit la personne a construire la sociétésujet, société authentiquement morale que nous postulons comme responsable et dotée de devoirs, il est logique de situer cette création de la société dans l'histoire. Nous sommes ainsi ramenés, de la question intemporelle du Bien et du Mal, à l'expérience individuelle de l'existence. Le plus important, alors, est moins de savoir comment se référer à un devoir universel et absolu, que d'imaginer ce qu'il faut faire, hic et nunc. Ceci ne va déjà pas sans difficultés dans un monde stable et permanent; mais c'est une question beaucoup plus hasardeuse quand on doit faire face à des changements profonds comme ceux que connaît le monde contemporain. En période de crise, en effet, le plus important n'est pas tellement de faire son devoir que de le discerner et de le définir. Certes, l'obligation demeure permanente, avec toutes les implications que nous avons dégagées dans ce travail, mais il importe avant tout de faire des choix dans les situations particulières de la vie. 
L'obligation sociale est toujours historique. Elle s'inscrit dans l'évolution d'une société à l'intérieur de laquelle la moralité naît par des conflits de devoirs, des choix héroïques ou des erreurs, une remise en question permanente des principes. Mais quelle que soit l'orientation de cette histoire issue des multiples choix individuels, ils construisent, par leur interaction, l'histoire des hommes et de leurs sociétés. Entrer dans la temporalité, ce n'est donc pas simplement accepter le poids de l'histoire, c'est la faire.

Dans cet horizon temporel, l'obligation représente non seulement une prise de conscience événementielle du sujet par lui-même, mais son insertion dans une histoire qui devient, par chacune de ses interventions, l'histoire de toute l'humanité. Or c'est justement parce que l'action ne peut pas être enfermée dans le seul champ abstrait d'un universel rationnel immuable, que l'histoire morale de l'humanité est appréhendée, saisie, comprise (au sens Jungien) par chacun des agents qui y engagent leur responsabilité. Il serait donc vain de tenter de proposer une éthique, même fondée sur l'universalité de l'obligation, qui ne tienne pas compte de la relativité des situations historiques. L'obligation est à l'origine de la société-sujet et de la moralité car elle agit dans l'être changeant du devenir. Comme le dit Hans Jonas, «c'est l'avantage de l'obligation de rendre compte du fondement métaphysique du devoir être». ${ }^{1}$ Ainsi, chacun des actes par lesquels nous nous construisons nous-mêmes participe au devenir de l'humanité.

Mais l'obligation peut-elle, elle-même, donner un sens à l'histoire? Pour répondre à cette question, nous devons distinguer l'obligation personnelle de l'obligation sociale. Si la personne est responsable d'abord de son propre devenir, que Max Scheler appelle sa vocation, cette personne n'est pas située historiquement dans un vide qu'elle serait appelée à remplir, par un acte pur d'invention ou de création. Elle naît, se développe, vit et meurt dans un monde préexistant, organisé, fait de groupes humains divers et de sociétés multiples, marqués eux-mêmes par les nombreux héritages de leur histoire antérieure, chargés des appels d'autrui qui ont précédé, et lourd des appels anticipés des générations futures. Tandis que l'obligation personnelle reconstruit l'être avec chaque vie personnelle, l'obligation sociale s'inscrit, elle, dans un mouvement historique qui dépasse la personne.

Certes, la société-autrui qui lance l'appel de l'obligation à la personne est inachevée, sinon elle ne connaîtrait aucun devenir; mais elle préexiste, dans son état d'inachèvement, à l'apport de notre propre contribution. Elle est une société-objet, faite de contraintes, que l'appel de l'obligation doit progressivement transformer en société-sujet au sein de laquelle, par la valeur, la personne doit s'épanouir. Nous ne pouvons dès lors ni ignorer son existence empirique et ce qu'elle nous impose, ni renoncer à la transformer par l'invention de nos valeurs. Notre obligation, dans l'histoire, fait de nous autant des héritiers que des fondateurs. En ce sens, nous contribuons

1 Hans Jonas, Le principe responsabilité, p. 71 
concrètement à donner un sens à l'histoire, mais ce sens ne peut pas être entièrement neuf. Comment le sujet peut-il se situer lui-même dans cette évolution?

Il en est du sens de l'histoire comme de la valeur: il est à la fois objectif et précaire, donné par l'expérience et réinterprété par le sujet qui le découvre. Il n'y a donc pas un sens empirique de l'histoire, mais une valeur du sens de l'histoire que je dois faire mienne pour que mon héritage ait une signification morale. Dans chacune des situations personnelles où je réponds à l'obligation, il faut que je puisse retrouver le sens d'une histoire universelle, sinon mon obligation ne m'ouvrirait pas vers l'humanité en général, mais serait limitée à définir pour moi un rôle partiel dans le cadre fermé du groupe humain, de l'organisation et de l'Etat dans lesquels je poursuis ma vocation: famille, race, religion, entreprise, nation, etc.. Car les sociétés sont multiples et c'est par rapport à toutes celles qui me concernent que je dois me situer.

C'est donc à moi de trouver, dans la situation individuelle de mon histoire personnelle, le sens qui me rattache à l'universel. Dans l'humanité prospère de cette fin de XXe siècle, j'ai le devoir de chercher un sens à l'histoire de tous les hommes pour être capable de discerner mon propre devenir. Le "retour du sujet» signifie donc une ouverture vers l'universel; car les choix individuels n'ont finalement de valeur morale que s'ils engagent toute l'humanité. Il faut, pour cela, être conscient du paradigme global qui caractérise nos sociétés, de l'ensemble des mythes qui les nourrissent et du mythe originel qui inspire l'activité humaine décisive qui caractérise notre temps, afin de découvrir, comme dit Raymond Aron, ala conception générale de l'histoire». C'est dans cette perspective que nous pourrons jeter une nouvelle lumière sur les fondements d'une éthique nouvelle libérée.

\section{Rupture symbolique et conscience de l'humanité}

\section{Au commencement était la valeur!}

Tout s'enracine dans cette certitude. Le récit mythique de la création du monde, tel que la civilisation occidentale l'a hérité, n'apporte plus, à première vue, un langage qui ait du sens pour les sociétés prospères et technologiques de cette fin de siècle. Il y a, dans l'histoire de la Genèse, quelque chose qui ne correspond plus aux valeurs contemporaines de la modernité. Pourtant, une relecture des légendes qu'il contient, dans la perspective du conflit de valeurs qui bouleverse notre époque, peut apporter une orientation utile pour «comprendre» la nature de notre obligation historique.

Un des récits de la création, tels qu'ils nous ont été transmis par la Bible, nous dit comment Dieu a créé le monde en sept jours, et comment, après avoir distingué la lumière des ténèbres, séparé la terre de l'eau, rempli les 
airs d'oiseaux et couvert la terre de plantes et d'animaux, après avoir surtout créé l'homme à son image et l'avoir placé, comme un phare, au sommet de la création en lui confiant la tâche d'attribuer un "nom» à tous les existants, - c'est-à-dire de maîtriser l'objet par la puissance du verbe sur les choses -, Il a contemplé ce qu'Il avait accompli et s'est déclaré satisfait de son travail. Dieu contempla tout ce qu'Il avait créé, et fut satisfait de ce qu'Il a vu; Il l'a trouvé «bon». (Genèse: 1: 30-31)2. Par ce récit mythique, l'homme, créé selon le texte à l'image de Dieu, imagine à son tour un Dieu à sa ressemblance et transfère dans l'être créateur sa propre façon d'appréhender et de ressentir le monde. Ce n'est pas dans la réalité objective de la création que se trouve la satisfaction de Dieu, mais dans le sentiment subjectif de satisfaction qu'Il éprouve à la vue de l'objet accompli. Le jugement de Dieu sur le monde est un jugement de valeur. Dieu, alors que l'homme est encore incapable de le faire, déclare que le monde est "bon à ses yeux»; il est subjectivement satisfait de contempler ce qu'Il voit. Dieu apporte la valeur au monde. Il y a valeur parce que Dieu est sujet, de la même façon que l'homme est sujet, ou l'inverse si l'on préfère: l'homme est sujet à l'instar de Dieu; car il y a nécessairement réciprocité dans toute ressemblance ${ }^{3}$-. Il est donc présent, en tant que sujet, dans l'acte par lequel il juge le réel. Le monde n'est pas bon en soi, il l'est par le jugement de Dieu.

Cette «bonté» du monde, cependant, comme toute valeur, est précaire. Ce sera le péché de l'homme de mettre en doute ce jugement divin. Car Adam et Eve mangent du fruit de l'arbre de la connaissance du bien et du mal, et deviennent à leur tour capables de juger, et d'exprimer leur propre valeur. C'est ce qui les rend semblables à Dieu. Regarde, dit Yaweh, l'homme est devenu semblable à nous, avec sa connaissance du bien et du mal. Il ne faut donc pas qu'il puisse étendre sa main pour prendre, en plus, le fruit de l'arbre de vie qui lui donnerait l'immortalité. Yaweh chasse donc l'homme du jardin d'Eden pour l'empêcher d'être pleinement Dieu. (Genèse, 3; 22-23). C'est donc la capacité de prononcer un jugement de valeur qui rend l'homme, non pas Dieu, mais «semblable» à Dieu. L'homme devient ainsi un dieu mortel, temporaire et passager, prisonnier d'une vie qui se termine avec la mort, mais «divin» tout de même par sa capacité de connaître le bien et le mal, et d'être, comme Yaweh devant sa création, satisfait ou insatisfait de ce qu'il contemple. Il devient à son tour, comme dit Sartre, «l'être par qui la valeur vient au monde». Or Adam et Eve ne sont pas satisfaits du monde qu'ils découvrent. A peine ont-ils mangé le fruit

2 L'utilisation que nous faisons ici des récits de la Genèse n'est pas fondée sur une exégèse scientifique ou une analyse de nature théologique, mais simplement abordée sur le mode symbolique. Notre «interprétation" ne doit donc pas être prise pour autre chose. Les traditions différentes qui expliquent ces différents textes ne sont pas analysées en elles-mêmes. Notre but est plus illustratif qu'explicatif.

3 L'on sait aussi qu'un Dieu monothéiste et créateur, donc distinct du monde, est une personne puisqu'il est unique et existant par soi! 
défendu, alors qu'ils se trouvent encore dans le jardin d'Eden, qu'ils se découvrent nus et ils ont honte. Le jugement qu'ils portent sur la nature dans laquelle ils sont incarnés n'est pas identique à celui de Dieu. Entre l'homme et Dieu il y a dès lors un conflit de valeur au sujet du monde créé et de la nature.

La rupture accomplie au jardin d'Eden est un mythe qui peut nous servir d'instrument d'interprétation de l'histoire humaine, et invite à voir dans cette dernière la longue tentative de réconcilier les jugements de valeur qui séparent Dieu et l'homme par rapport au monde. Il y a trois termes qui jouent un rôle dans ce conflit: Dieu, aux yeux de qui le monde est bon; l'homme, insatisfait dès qu'il juge le monde selon son propre pouvoir de juger; et le monde, le réel donné, l'objet des jugements de valeur contradictoires de l'homme et de Dieu. Le mythe du péché originel est le symbole de cette rupture, un événement, - donc une entrée dans l'histoire - tragiquement marqué par la condamnation de l'homme par Dieu, et le rejet de l'homme du monde bienheureux et stable dans lequel il avait vécu avant la connaissance des valeurs. La rupture originelle appelle une action pour la corriger. Ce doit être une action humaine, portée par toute l'humanité issue du mythe d'Adam. Mais l'action n'est jamais abstraite et universelle. Même si elle demeure symbolique, elle doit se concrétiser dans des actes réels, historiques, identifiables. On va donc la retrouver dans les manifestations historiques des sociétés, des groupes humains et des individus dans lesquelles l'humanité s'incarne dans le temps.

Trois grandes orientations s'ouvrent à l'action humaine pour parvenir à réconcilier les termes du conflit et retrouver le bonheur brisé. Chacune d'elles se rapporte à l'un des trois termes composant le conflit. On les rencontre, au cours des siècles, tantôt conjointement, tantôt successivement, tantôt conflictuellement. Le choix spécifique de l'une ou de l'autre, ou la nature de leur interaction, fournissent d'ailleurs des moyens d'interpréter la diversité empirique des civilisations.

La première voie est celle de l'éthique, de la recherche de la sagesse, de la transformation (ou correction) des comportements humains, et de la moralisation de la société (et de la politique), qui consiste à agir sur l'homme pour tenter de corriger le jugement humain, de le redresser et de supprimer le conflit qui l'oppose au jugement de Dieu. La voie éthique a pour but de changer l'homme, individuel et collectif, pour qu'il se comporte selon ce que Dieu, ou la société, attendent de lui, comme si la faute n'avait pas été commise. Dieu n'a plus alors à condamner un homme réhabilité par sa transformation morale. On retrouve cette orientation dans les lois hébraïques de Moïse, reçues de Dieu sur le Sinaï, qui montrent la voie que Dieu propose à l'homme pour se transformer. C'est aussi la voie des sagesses purement humaines, comme les sagesses orientales, qui mènent le sage vers une forme de fusion avec la divinité panthéiste, ou celle des sagesses de l'antiquité, qui visent une réconciliation dans la paix de l'homme avec lui-même. Les 
idéologies poursuivant l'idée d'un bonheur humain s'identifiant à l'idéal d'une société parfaite se rapprochent également de cette tendance. Cette transformation, comme nous l'avons vu, peut avoir lieu par la contrainte, la coercition politique de la société-objet, mais aussi par la conviction interne et la libre adhésion de l'individu à l'idéal moral proposé par l'obligation. Première voie, donc: agir sur l'homme.

La deuxième doit permettre d'éliminer la condamnation divine en agissant sur Dieu lui-même. C'est la voie religieuse. C'est surtout celle des religions rituelles, sacrificielles et de salut, qui vise, par une action humaine, souvent inspirée ou suggérée par Dieu (toutes les religions révélées s'appuient sur cette intervention divine), à pénétrer le monde sacré du surnaturel et à transformer l'attitude du créateur. Ceci se produit par l'offrande à Dieu d'actions humaines ou d'objets spécifiques qui Lui plaisent: prières, sacrifices, rites propitiatoires, offrandes sacrées, cérémonies et cultes capables d'amadouer la colère de Dieu, de réparer l'offense du péché originel et d'attirer sa bienveillance. Les religions animistes, qui s'appuient avant tout sur l'idée d'une manipulation possible des esprits divins, sont des exemples évidents de cette orientation. Mais le christianisme est lui-même l'exemple historique qui correspond certainement le mieux à cette démarche puisqu'il fait du sacrifice temporel de Jésus l'acte suprême qui réconcilie le Père éternel avec ses enfants. Certes, dans le christianisme, c'est toujours Dieu qui agit sur lui-même par amour pour l'homme qu'il rachète par son fils, mais il faut un sacrifice venant de l'humanité elle-même. C'est donc l'incarnation du Nazaréen qui permet à l'homme pécheur de ne plus craindre la colère de Dieu.

La troisième voie consiste à transformer matériellement l'objet même du jugement de valeur, et à tenter d'en faire une réalité qui ne déplaise plus. Cette transformation du monde est technologique et matérielle. Elle a pour but de corriger et de reconstruire le monde pour qu'il corresponde plus étroitement à l'image que l'homme pouvait s'en faire avant la rupture symbolique, ou en dépit de cette rupture. C'est une tentative pour reconstituer, progressivement, un monde qui soit aussi idéal que le jardin perdu, un monde dans lequel la «honte de la nudité» ne soit plus un fardeau. Ce fut d'ailleurs, selon le mythe de la Genèse, la première réaction d'Adam et Eve puisque, honteux d'être nus, ils se sont aussitôt couverts.

Adapter la réalité du monde au jugement de valeur posé par l'homme est une action humaine. Elle fait appel à la créativité, la technologie, l'économie, toutes les forces physiques et intellectuelles de l'homme. Elle demeure cependant une action exclusivement matérielle qui adapte l'environnement et qui, pour ainsi dire, englobe l'homme dans un environnement nouveau. Cette troisième voie caractérise plus spécifiquement l'histoire des sociétés occidentales et explique, dans une large mesure, la situation d'aujourd'hui.

Ces trois voies «mythiques» de l'histoire ont joué en permanence leur rôle dans le développement du monde. L'histoire, en effet, peut être 
interprétée comme l'interaction, parfois incohérente et conflictuelle, parfois plus ou moins harmonieuse, de ces trois différents domaines de l'action:

- l'action sur l'homme par la recherche de la sagesse, l'amélioration des comportements humains et la construction de la société idéale,

- l'action sur Dieu par le recours au sacré et au surnaturel, et

- l'action sur le monde et la nature par la transformation matérielle de l'environnement.

Nous sommes arrivés, apparemment, à un stade de l'histoire humaine où la troisième voie semble avoir globalement supplanté les deux autres et où les succès mêmes de l'économie et de la technologie conduisent à mettre de côté les voies morale et religieuse

\section{Sociétés et sujets dans la construction de l'histoire}

Si l'histoire de l'humanité se confond aujourd'hui avec celle du développement de la technologie, il est évident que cette évolution récente est le résultat direct de l'accumulation des succès de l'histoire économique récente, de l'industrialisation et du capitalisme. Histoire technologique et histoire économique se rapprochent et s'identifient, et leur évidente interdépendance conduit l' humanitéà reconsidérer l'agir humain de manière entièrement nouvelle.

Les trois voies ouvertes par la rupture symbolique originale ont suscité trois domaines distincts de l'action humaine dans l'histoire. Or ces trois domaines, qui se sont maintenus plus ou moins séparés au cours des siècles, requièrent des types différents de sociétés ou d'organisations, entraînant des comportements spécifiques adaptés aux buts poursuivis. Ces trois domaines manifestent également des formes particulières de pouvoir, et le rôle qu'ils jouent dans la société globale dépend du pouvoir relatif de chacun d'entre eux sur les autres.

Ces trois domaines ont donné naissance également à différentes formes de poursuite individuelle et collective du bonheur et des conceptions différentes de la finalité humaine. Tandis que la religion promet un bonheur dans l'au-delà (bonheur transcendant), Dieu étant la fin ultime de l'homme, la morale situe le bonheur dans la sagesse intérieure de l'homme et dans l'accomplissment de son être (bonheur immanent), alors que l'économie et le pouvoir technique promettent une satisfaction dans la possession des richesses terrestres et la domination de la matière (bonheur social englobant). L'existence conjointe, dans notre tradition culturelle, de ces trois domaines de l'action humaine a eu pour premier effet d'introduire, dans les structures sociales, une distinction nette entre les sociétés, institutions ou groupements humains dans lesquels s' incarnait la poursuite des trois bonheurs mentionnés ci-dessus. 
Il y a d'abord eu la distinction évidente entre la cité de Dieu et la cité terrestre, accomplie par la séparation de l'Eglise et de l'Etat, qui a caractérisé l'évolution politique de l'Occident au moyen-âge. Ce fut le premier pas vers l'affirmation de l'autonomie de la vie politique, qui a permis un cheminement historique conduisant progressivement à la libéralisation de la vie politique elle-même, à la reconnaissance de la responsabilité de l'individu dans ce domaine, et finalement aux formes de démocraties libérales de notre époque. ${ }^{4}$ L'industrialisation et l'histoire du capitalisme conduisent eux aussi à une distinction importante en séparant progressivement l'activité économique et technique de la société politique.

Aujourd'hui, la société-sujet que l'obligation nous appelle à construire apparaît de plus en plus comme une organisation distincte de la société générale, plus volontaire et participative. Notre époque est celle dans laquelle la mondialisation de la société humaine se produit conjointement avec la multiplication des organisations et groupements volontaires dans lesquels le sujet peut chercher l'accomplissement de sa vocation.

On a constaté, d'autre part, une évolution des sociétés dans l'histoire, tant des structures religieuses que des structures politiques, vers une plus grande individualisation. Les sociétés ont apparemment passé d'un statut avant tout contraignant, société-objet, vers des formes sociales plus respectueuses du sujet et de son autonomie et de son rôle actif, société-sujet. L'individualisation progressive de la conscience religieuse et la démocratisation croissante de la vie politique en sont la preuve. Malgré la survivance de nombreux archaïsmes, le despotisme n'est plus la forme dominante des sociétés religieuses et politiques; on y retrouve,- encore imparfait, certes - un respect du sujet qui a transformé, dans la plupart des cas, le fonctionnement et les finalités de ces sociétés. La conquête récente de la liberté de conscience, des droits de l'homme et de la participation démocratique des citoyens, montre bien comment le sujet pénètre le processus d'évolution sociale et marque progressivement l'histoire de sa présence. L'idée d'un retour du sujet n'est donc pas entièrement nouvelle. Religion et morale ont déjà évolué dans ce sens.

Nous avons montré, dans les deux premiers chapitres de ce travail, que le domaine de l'action technologique et économique dominait indéniablement notre époque, que ses organisations spécifiques tendaient à occuper tout l'espace social, de même que le paradigme des comportements qu'il exigeait visait à remplir tout l'espace moral. Nous avons montré comment le monde des valeurs était envahi par l'éthique de la prospérité. Nous assistons, non sans observer quelques réactions de défense désordonnées, à un glissement du pouvoir dans la société, à partir des organisations et des institutions religieuses, morales et politiques, vers une société quasi exclusivement

4 Voir, à ce sujet, Gabriel Fragnière, Le Royaume de l'homme 
économique ${ }^{5}$. Or ce nouveau pouvoir, dû au succès incontesté de l'invention humaine dans le processus de transformation de la nature, se présente aujourd hui comme une menace et une contrainte. On a l'impression, que par son pouvoir immense de persuasion et de motivation des énergies humaines, la société-objet de l'économie s'impose.

C'est devant cette évolution que nous en appelons à la conscience morale du sujet qui doit faire évoluer la société vers une société-sujet dans laquelle la personne trouvera son espace de sujet libre et responsable. Nous devons faire appel à une sorte de nouvelle rupture symbolique en faveur de la valeur.

Si un certain équilibre entre les trois voies de réconciliation de l'homme, de la nature et de l'absolu est rompu au bénéfice du seul pouvoir économique, l'homme risque de se retrouver prisonnier de son propre pouvoir, au sein de l'instrument qu'il a développé pour transformer la nature, et de dépendre de l'action économique comme il dépendait de son créateur dans le jardin d'Eden, avant d'acquérir pour lui-même le pouvoir de la valeur. Le succès de l'inventivité technologique humaine enferme l'homme dans un bonheur englobant qui peu à peu l'aveugle et menace sa liberté. L' auto-satisfaction de la vieéconomique florissante peutêtre imaginée, àl'instar de la satisfaction du Dieu créateur de la Genèse qui imposait son propre jugement de valeur sur un homme béatement irresponsable, comme une nouvelle force dominante qui pèse de tout son poids sur les jugements humains, en présentant à I'homme la tentation d'une vie bienheureuse dans quelque nouveau jardin d'irresponsabilité appelé prospérité illimitée.

C'est pourquoi nous pensons que le retour du sujet, face à cette menace, doit être présenté comme la conquête d'un nouveau «fruit défendu», volé au dieu de la richesse matérielle, et source d'une nouvelle rupture symbolique. Il faut donc un nouveau serpent pour que l'homme soit motivé à renouveler son geste. Nous croyons que cette motivation peut venir d'une prise de conscience de la menace qui pèse sur l'homme.

Hans Jonas écrit: "La frontière entre "Etat» (polis) et «nature» a été abolie: la cité des hommes, jadis une enclave à l'intérieur du monde non humain, se répand sur la totalité de la nature terrestre et usurpe sa place. La différence de l'artificiel et du naturel a disparu, le naturel a été englouti par la sphère de l'artificiel; et en même temps l'artefact total, les oeuvres de I'homme devenues monde, en agissant sur lui-même et par lui-même, engendre une nouvelle espèce de «nature», c'est-à-dire une nécessité dynamique propre, à laquelle la liberté humaine se trouve confrontée en un sens entièrement nouveaum. ${ }^{6}$ En transformant le monde pour le rendre satisfaisant à ses désirs, l'homme y a été progressivement englouti.

5 On retrouve une argumentation allant dans ce sens dans: Robert Reich, The Work of Nations.

6 Hans Jonas, op. cit., p. 29 
C'est en effet par rapport à cette situation nouvelle que doit être être posé le choix moral. Discerner notre devoir consiste donc à reconnaître notre responsabilité par rapport au monde que l'histoire a créé, en particulier l'histoire technico-économique récente. Hans Jonas indique que nous avons certainement à souhaiter qu'un monde "approprié à l'habitation humaine» existe dans l'avenir; seulement il n'est pas sûr que ce monde puisse garantir l'existence de l'humanité. Cela pose le problème de choisir nos devoirs prioritaires devant les conséquences de nos nouveaux pouvoirs. "La présence de l'homme dans le monde était - [autrefois, c'est-à-dire avant la révolution technologique contemporaine] - une donnée première, ne posant pas question, d'où toute idée de l'obligation dans le comportement humain prenait son départ. Désormais elle est devenue elle-même un objet d'obligation - à savoir l'obligation de garantir pour l'avenir la première prémisse de l'obligation, c'est-à-dire justement la simple présence de candidats pour l'existence d'un univers moral au sein du monde physique; et cela veut dire entre autres préserver le monde physique de manière que les conditions d'une telle présence restent intactes; et cela veut dire protéger leur vulnérabilité contre la menace d'une atteinte portée à ces conditions».?

Le premier devoir de notre historicité est donc bien de garantir, dans le monde actuel, la présence de personnes susceptibles de maintenir un univers moral au sein du monde physique. Cela signifie: intervenir moralement dans la société technico-économique dominante. Cette exigence est le retour du sujet, et l'obligation morale nous apparaît comme l'instrument pour y parvenir.

Nous retrouvons ce besoin sous une autre forme au terme de cette réflexion sur l'historicité de notre condition. Pour reprendre les termes de la symbolique que nous avons retenue, nous devons réanimer la sphère de l'action éthique face à la toute puissance de l'action matérielle. Il faut retrouver l'immanent pour se défendre contre l'englobant. Car il serait dangereux, pour l'homme, de renoncer à agir sur lui-même devant le succès irrésistible de son action sur le monde. L'histoire perdrait son sens si elle était réduite à sa dimension technologique. La première obligation envers le monde est donc morale, ce que nous appelons la conscience de l'humanité, et que Jonas nomme le «Principe responsabilité».

On ne change pas le monde en renonçant à changer l'homme. Il faut changer l'homme, car son existence est essentielle à la valeur du monde. Au début était la valeur; c'est par elle que le monde a acquis son importance aux yeux de l'homme. C'est le conflit que la valeur a fait naître entre l'homme et Dieu qui explique notre entrée dans l'histoire. Renoncer à l'univers moral, aujourd'hui, signifierait rejeter l'obligation de placer l'homme au centre de l'histoire, et par conséquent y mettre fin.

7 Ibid., p. 29-30. C'est nous qui soulignons 


\section{La conscience de l'humanité est aujourd'hui}

le premier devoir vis-à-vis du monde.

Ce devoir consiste à refuser toute formule qui conduirait à voir le monde subsister sans l'homme. Le monde n'aurait dans ce cas aucune valeur et son histoire n'aurait pas de sens. Il faut, pour cela, réintroduire l'univers moral dans la sphère toute puissante de l'action technico-économique.

Ladeuxième idée, exprimée aussi par Jonas, est de protéger la vulnérabilité des personnes qui assurent l'existence de cet univers moral, en garantissant, en vue de leur présence dans le monde en tant que sujets, les conditions de cette existence. La conscience de l'humanité nous demande de garantir à chacun la possiblité de vivre moralement comme un homme.

C'est à cette exigence que nous rattachons l'idée de Justice.

\section{La Justice: de la théorie à la valeur}

Il semblerait évident, à première vue, que le premier effort de tout théoricien de la justice fût de construire un système cohérent de la justice qui emportât la conviction sur la base d'idées indubitables. Celles-ci, en effet, font appel à la raison, l'intelligence et la logique pour en justifier la mise en place. C'est, semble-t-il, le point de départ de John Rawls, dans son ouvrage très discuté: Théorie de la Justice. Il s'appuie en effet sur l'idée d'une position originelle dont l'équité entre des personnes égales est garantie en raison de sa nature totalement fondamentale. ${ }^{8}$

Toutefois, l'expérience montre qu'il ne suffit pas que la justice existe en elle-même dans quelque ciel platonicien, ou dans un état de nature préexistant à l'expérience historique, il faut qu'elle soit appliquée à des cas concrets pour être réelle. Or c'est justement ce besoin d'introduire la justice dans l'existence journalière des individus qui est à l'origine de sa relativité, nous pourrions aussi dire de sa précarité. Avant même de discuter de sa nature, la naissance et l'existence de la justice comme valeur doit être mise en question. Certes, si la justice en tant que concept et en tant que "première vertu des institutions sociales»" ne peut être décrite que dans l'absolu, on ne peuten fairel'expérience que dans des incarnations concrètes et individuelles, nécessairement limitées, qui trouvent également leur place dans l'histoire. La justice est donc essentiellement une valeur morale.

L'anecdote du roi Salomon, proposant de trancher en deux le corps d'un enfant revendiqué par deux mères, est devenue un symbole de la justice précisément parce que la justice qu'il propose s'incarne dans un acte bien

8 Voir John Rawls, Théorie de la justice, (1971) (1987)
9 lbid., p. 29 
précis, dont les conséquences, s'il avait été commis, eussent représenté pour l'enfant sacrifié le sommet de l'injustice. Il y a, dans cette histoire biblique, une compréhension directe de la justice, une appréhension quasi spontanée, pré-rationnelle, qui nous fait sentir la justice, plutôt que de nous en donner une connaissance abstraite au niveau des principes nécessaires. La justice de Salomon est celle qui intègre d'autres valeurs morales: la vérité, l'amour maternel, la victoire de la vie. Salomon, dans ce cas précis, ne prononce pas un jugement de justice équitable, il donne l'occasion à la vraie mère de révéler la justice par l'amour de son enfant qu'elle manifeste en préférant l'abandonner plutôt que de le voir mourir. La justice, dans ce cas, n'est pas rendue par l'application d'un principe, mais par un risque humain.

Cette appréhension directe de la justice remplace, pour ainsi dire, dans la prise de conscience du conflit qu'elle révèle, toutes les théories les plus élaborées auxquelles il est envisageable de se référer. C'est pourquoi nous disons qu'il ne suffit pas de construire un système de la justice pour faire comprendre ce qu'elle implique, il faut la saisir hic et nunc dans chacune des situations qui la mettent en question.

Or, à l'instar des concepts moraux que nous avons analysés, la justice se présente tout d'abord comme un concept négatif. «Ce n'est pas la justice qui motive l'action, mais l'injustice et le mécontentement». ${ }^{10} \mathrm{Il}$ semble plus facile de l'exiger quand elle manque que de dire ce qu'elle est. Il y a, dans la notion de justice, une dimension qui en fait un concept de la vie pratique et l'objet d'une demande. «Tandis que nous pouvons solliciter ou espérer l'action d'autrui bienveillante, généreuse ou solidaire, la pratique juste est quelque chose que l'on exige.... En présence de l'injustice ...l'on est indigné et révolté, et l'on proteste. Les hommes ne se recommandent ou ne suggèrent pas simplement les uns aux autres d'accomplir l'obligation sociale qu'est la justice, mais la réclament, l'exigent. ... c'est à la justice que revient la priorité à l'intérieur de la morale sociale et peut-être est-elle seule légitime pour un ordre social qui a un pouvoir de contrainte»."

Le problème de la justice est donc avant tout un problème moral, et non pas une question de philosophie pure, de droit fondamental ou appliqué, ni même d'institutions politiques. Certes, la justice est politique, car sa dimension sociale est évidente et elle joue un rôle essentiel dans le pouvoir contraignant des Etats, mais elle est avant tout morale car elle concerne le sens de l'action humaine. En effet, dans la mesure où la justice n'existe que dans les cas où elle s'applique, c'est-à-dire n'a de sens que par les individus réels qui sont affectés par son absence, elle revêt également cette ambiguité qui caractérise la morale elle-même; elle concerne à la fois le sujet dans son existence individuelle, et une norme qui échappe au sujet parce qu'elle est essentiellement sociale. Comme le Bien et le Mal, la justice doit exister pour

10 Kenneth E. Boulding, The Organizational Revolution, p. 83

11 Otfried Höffe, La justice politique, p. 40-41 
tout le monde. Elle ne s'impose pas comme une évidence, elle suscite la révolte par son absence.

Voilà pourquoi il n'est pas possible de concevoir la justice comme une perfection établie ou la fin d'une organisation sociale. Il y a toujours, au delà de l'établissement de la justice, une autre fin humaine que la justice doit soutenir. La justice doit permettre, selon la citation de Hans Jonas, aux personnes prises individuellement de maintenir la présence de l'univers moral au sein du monde physique, c'est-à-dire elle est un «moyen moral», objet d'une obligation sociale, qui permet à l'homme-sujet de vivre son obligation. Elle est la condition première du retour du sujet dans la vie sociale. La justice est donc ordonnée aux personnes, et non l'inverse.

Si la justice se présentait comme fin, les sociétés individuelles seraient tentées de correspondre à sa définition et de prétendre posséder par elle une perfection pratique en fonction du degré de sa matérialisation dans leur type d'organisation. Certes, on sait qu'il existe, empiriquement, des sociétés dont on dit qu'elles sont plus ou moins justes. Mais le critère de justice n'est jamais, dans ces cas, fondé sur l'observation des données abstraites de la vie sociale, mais sur le comportement des individus; les sociétés «injustes» sont celles où la contestation de la vie sociale est la plus virulente. C'est toujours dans l'attitude humaine envers la société que se reflète le degré de justice sociale, et non dans la correspondance des structures sociales avec un modèle purement abstrait. La justice, comme la morale, résulte d'un jugement de valeur.

Nous nous appuyons ici sur une autre expérience de la temporalité humaine. Il est courant que l'homme se révolte devant la présence d'une trop grande injustice, mais à aucun moment une société n'a le droit de dire qu'elle a accompli la summa justicia en son sein, car elle pourrait toujours être contestée par une nouvelle conscience. Le critère de la justice se trouve donc dans le sentiment de la justice, et non dans un type objectif d'organisation. La justice est ainsi, fondamentalement, une valeur.

Mais c'est une valeur qui ne s'exprime jamais au nom d'un seul individu; elle doit toujours l'être au nom de l'universel. De la même façon, dans les sociétés et organisations de ce monde, c'est toujours par l'appel à l'universel que le sujet individuel retrouve sa place au sein des structures. C'est ce que nous appelons la conscience de l'humanité, par laquelle le sujet rétablit ses droits dans le fonctionnement du groupe.

Universelle comme valeur, la justice est toujours multiple, et ceci en plusieurs sens. D'abord en elle-même comme concept, car elle doit être à la fois un idéal et une solution concrète. Mais elle est aussi et avant tout multiple dans sa dimension sociale, car elle se présente d'une part comme une norme qu'il faut poursuivre, et d'autre part comme une valeur précaire, souvent contradictoire jusque dans son application, puisqu'elle peut être d'une part un bénéfice pour celui qui en profite, et d'autre part une limitation pour celui qui la subit. 
C'est pourquoi la construction de la justice ne peut être qu'un processus historique, un développement qui suit le rythme de la prise de conscience de ses exigences par les sociétés humaines. Sur le plan politique, nous dirons que l'histoire de la justice s'identifie avec le développement de ce que nous appelons aujourd'hui les «droits de l'homme» et le progrès de la démocratisation des organisations politiques. Il n'y a d'ailleurs pas d'exemple plus symptomatique du retour du sujet que le recours à des formes toujours plus décentralisées de participations des citoyens à la vie politique.

La relativité temporelle de la justice est souvent source de sa faiblesse. C'est pour cette raison que ce qui est aujourd'hui juste, à nos yeux, pouvait très bien être ignoré des générations précédentes, ou leur paraître comme ne ressortissant d'aucune façon au domaine de la justice. On pourrait citer ici Jefferson, prophète des droits de l'homme, auteur de la déclaration d'indépendance des Etats-Unis d'Amérique, qui non seulement tolérait l'esclavage des noirs, mais allait même jusqu'à le justifier. Comme la liberté, la justice n'est comprise que dans son devenir. Elle est une idéeforce, elle n'est jamais un état, ni une idée claire.

Nous verrons, dans le Chapitre suivant, que toute individualisation de l'action humaine se présente comme une chance de progrès de la moralité; ainsi toute prise de conscience d'un rapport plus étroit entre la norme sociale de la justice et son impact sur l'existence concrète des personnes représente également une chance de progrès de la justice. Il n'existe pas la justice d'un côté, ressortissant à la philosophie et au droit, et l'éthique de l'autre, qui dirige les actions humaines individuelles. Il doit y avoir une éthique de la justice, c'est-à-dire une obligation de recourir à la justice pour favoriser le développement des personnes, et c'est en ce sens qu'elle est entièrement morale, formellement et matériellement, $c$ 'est-à-dire essentiellement imprègnée de la subjectivité de l'agent, et correspondant à une norme qui se découvre dans l'intersubjectivité des personnes.

Parler de la justice, aujourd 'hui, c'est donc parler de notre conscience historique de la justice comme valeur. La justice est un des éléments de la construction de l'histoire. Qu'en est-il donc de la justice et de la richesse?

\section{La distribution et le partage: justice et responsabilité}

La première question qui vient à l'esprit est celle de ce qu'on appelle couramment la justice distributive, et de son rapport aux finalités de l'éthique de la prospérité.

Si la richesse est conçue comme un bien disponible, et qu'il est possible, dans une certaine mesure, de la considérer comme un bien collectif, il semble à première vue évident que cette richesse doit être redistribuée entre tous les membres du corps social. Le droit de propriété ne doit pas favoriser, entre les individus, des différences et des déséquilibres qui peuvent être 
ressentis comme des manifestations d'injustice. La justice demande donc un système de redistribution. En effet, comme c'est l'existence du corps social et de son fonctionnement qui rend possible la production de la richesse, la jouissance de cette dernière doit être également redistribuée sur l'ensemble de la société. L'idée de justice est dans ce cas rattachée au plus haut niveau possible d'égalité entre les individus. Toute différence dans la jouissance de la richese pourrait être vue comme une injustice. La justice semble, à première vue, s'opposer à l'existence, dans un même groupe social, de riches et de pauvres. Comment donc aborder cette question?

Certes, l'utilitarisme libéral pense que cette redistribution doit être spontanée, et que les lois du marché sont suffisantes pour assurer les échanges entre tous afin de favoriser le plus grand bonheur «matériel» pour le plus grand nombre. Il ne faut donc pas se poser $a$ priori la question de la justice distributive, mais la laisser se mettre en place par elle-même. La «main invisible» y pourvoit! Cette croyance est suffisamment enracinée dans l'idéologie libérale actuelle pour être encore souvent défendue, alors que beaucoup la mettent en doute. Certes, la prospérité peut être relativement bien «distribuée» à l'intérieur de sociétés suffisamment riches pour financer des processus correcteurs d'injustice, mais les résultats éthiques des systèmes d'Etat Providence, ne résistent souvent pas aux difficultés économiques imprévues, ${ }^{12}$ et peuvent encore être à l'origine d'injustices plus graves quand certains moyens de redistribution à l'intérieur de pays privilégiés sont mis en place au détriment d'autres pays plus pauvres. La «main invisible» ne peut être appelée au secours de la justice si son impact se limite à quelques régions du monde et exclut l'idée d'une redistribution vraiment universelle. Comme nous l'avons souligné, la justice est une valeur qui ne peut se limiter aux intérêts du seul sujet, mais doit s'ouvrir à toute l'humanité. Il y a donc un danger d'injustice dans le «laisser faire» économique qui se fait souvent au détriment d'une compréhension de la valeur morale de la justice. "Croire ou faire croire que la liberté économique laissée à ellemême parviendra à résoudre les inégalités sociales, c'est supposer que la connaissance des inégalités engendre nécessairement son correctif. C'est au fond une attitude gnostiques. ${ }^{13}$

Nous pensons, de plus, que le dynamisme même de l'éthique de la prospérité, qui établit la valeur suprême dans la recherche d'un «toujours plus», peut grandement fausser la réflexion sur la redistribution. La justice risque, en effet, de se perdre en tant que valeur. D'abord, on pourrait défendre l'idée qu'une des premières exigences de la justice consiste à stimuler toutes les actions susceptibles de favoriser l'accumulation de la richesse, car il est plus juste, dans la perspective d' une éthique redistributive de la prospérité, que non seulement la richesse existante soit distribuée, mais

\footnotetext{
12 Voir à ce sujet Jean-Pierre Jallade, (ed.) The Crisis of Redistribution in European Welfare States

${ }^{13}$ Michel Schooyans, La dérive totalitaire du Libéralisme, p. 104-105
} 
que cette richesse croisse de façon permanente. La volonté de produire et d'augmenter le profit qui caractérise l'éthique de la productivité serait donc une première démarche favorable à la justice. La justice ne se contente dès lors plus de garantir le nécessaire, mais d'augmenter le disponible.

Mais cette fin justifie-t-elle toutes les actions qui la rendent possible? L'action bienfaisante, ex post, qui consiste à rendre accessibles à tous les fruits de la production, suffit-elle à rendre juste une accumulation préalable de richesses par des processus fondés sur le seul souci de la performance et de l'efficacité? L'idée même que le système de production ait pour but d'enrichir tout le monde doit-elle autoriser n'importe quelle forme de contrainte et d'exploitation? Une réflexion plus poussée sur cette question nous ramène à l'interrogation première de ce travail. Il n'est pas possible d'admettre que le critère quantitatif puisse justifier toutes les formes d'actions humaines au nom d'une justice qui serait favorisée par l'augmentation quantitative de ses moyens. La justice ne se trouve pas dans la seule redistribution quantitative de la richesse accumulée parl'activité économique, mais aussi dans l'utilisation que l'on fait des ressources humaines dans le processus de production. Il y a donc quelque chose d'a-moral dans la limitation de la notion de justice au seul aspect de la redistribution de la richesse. L'éthique de la prospérité ne permet pas de justifier tous les moyens. «Il est débile de penser que, par la seule vertu de sa réalité, la liberté économique engendre la justice ou que les requêtes de celles-ci sont honorées par la magie du seul contrat de travail». ${ }^{14}$

En tant que valeur, la justice ne peut donc être évaluée par les seuls critères quantitatifs de la croissance et de la redistribution, quels que soient d'ailleurs les systèmes de détournement des richesses disponibles qui ne parviennent pas toujours à ceux qui en ont le plus besoin..$^{15}$ La justice n'est jamais satisfaisante quand on veut l'établir par des mesures imaginées au nom de la seule quantité.

La valeur humaine, telle qu'elle se découvre dans l'affirmation du sujet, n'est pas quantifiable. C'est pourquoi nous pensons qu'en recourant à la notion d'obligation morale, il est possible de replacer l'action individuelle en dehors du contenu de l'action collective, et proposer, à partir de ce point de vue, une éthique de la justice qui soit fondée sur l'homme comme sujet, et non pas sur le simple calcul des effets quantitatifs et matériels de ses actes. Il ne suffit pas, en effet, de valoriser les seules causes finales des actions humaines pour parvenir à la notion de justice. Le fondement dernier de la

\section{Idem}

15 Voir par exemple, les théories sur «l'effet Matthieu» des systèmes actuels de redistribution dans les «Etats Providence», décrites par le Prof. Deleeck d'Anvers. On se réfère à l'évangile de Matthieu, dans lequel il est dit: il sera beaucoup donné à ceux qui ont déjà beaucoup reçu. 
justice se trouve finalement dans le sujet, comme fin, et non pas dans un équilibre quantifié de ses actes. Salomon rompt définitivement cette notion de ujustice-équilibre», selon laquelle chacun doit recevoir la même chose.

C'est pourquoi il n'est pas possible d'établir la justice sur la seule idée d'une égalité des conditions et des revenus. La distribution n'est pas le chemin qui conduit à la notion de justice, telle que nous la suggérons dans la perspective historique où nous nous situons, garantir la survie du sujet afin que l'univers moral ne soit pas éliminé de la vie sur cette planète. Plus proche de cette conception se trouve, croyons-nous, la notion de partage.

Si la justice n'est finalement pas: «à chacun la même chose», la formule: «à chacun ce qui lui est dû» est-elle plus exacte? La première objection à ce type de définition se trouve dans la notion même de sujet. Comment, en effet, établir une mesure objectivable de ce qui est "dû» à chaque sujet? Le devoir envers lui n'est-il pas celui que nous avons sans cesse rencontré dans ces pages, à savoir l'obligation de l'appeler à la valeur pour lui-même et pour le groupe? Si la première chose qui est «due» au sujet, est qu'il soit considéré comme tel, afin qu'il puisse découvrir sa propre vocation dans sa vie sociale et personnelle, ce que la société lui doit, n'est pas une certaine quantité dans la redistribution de la richesse produite, mais une vie et une considération personnelle qui fasse de sa vie propre une vie respectée et digne de sa propre vocation. Ce que la société lui doit, c'est une relation inter-subjective et inter-personnelle avec chaque autre membre du groupe, un respect de sa responsabilité et une vie pleinement vécue comme sujet. Des sujets peuvent ainsi partager leur expérience individuelle. C'est au niveau de ce partage que se trouve la justice capable de répondre au besoin de survie de l'humanité dans le monde.

L'éthique de la prospérité ne désigne la justice que par la mesure des biens qui circulent au niveau de la consommation: certes il n'est pas nécessaire que tous consomment la même chose, mais le principe de justice est fondé sur le principe que chacun a droit, selon ses besoins, à une part légitime de la richesse totale. Dans l'optique d'une éthique de la justice fondée sur notre idée de partage, le besoin fondamental ne se mesure pas en termes de quantité de consommation, mais en valeur de vie personnelle et sociale dans l'accomplissement de sa vocation.

La distribution ne concerne que des choses; le partage va au-delà de l'objectivité matérielle et concerne toute la vie.

Le partage ne concerne pas seulement le niveau de vie, mais la manière de vivre, les biens, les valeurs, les croyances, les enthousiasmes, la beauté, le bien et le mal, tout ce qui fait que la rencontre d'êtres humains implique l'entièreté de leur nature. Il n'y aurait pas de justice réelle si les relations humaines se limitaient aux quantités. C'est l'aspect le plus destructeur de 
la vie sociale que comprend l'éthique de la prospérité, et le détournement le plus évident de la notion de justice.

La dernière conséquence d'une justice distributive totalement quantifiée est la disparition, devant l'objectivité des nombres, de la responsabilité de l'individu envers autrui. Si chacun reçoit une part égale des biens disponibles, et que cette égalité est garantie par des mesures qui sont indépendantes de ma volonté, je ne suis responsable que de la masse qui me revient, et n'assume aucunement la responsabilité du partage avec autrui. La justice distributive m'enferme dans l'unique rôle de gestionnaire du patrimoine de biens qui me revient. Cette justice me coupe de ma responsabilité sociale, et nie mon obligation envers autrui. L'autre n'existe plus en tant que sujet, mais seulement en tant que membre d'une entité sociale par rapport à laquelle nous devenons les objets égalitaires d'une redistribution. La notion de partage, au contraire, m'engage dans ce que je donne et ce que je reçois, et ne nie pas l'existence de l'autre. Le devoir de justice n'est pas seulement une contrainte quantitative, il demeure une obligation "morale».

\section{L'Etat: sujet du devoir de Justice}

Mais suis-je le seul sujet de cette obligation morale, ou de ce devoir de justice? Cette question met en question le rôle de l'Etat, en particulier en raison de son caractère contraignant. L'Etat dit le droit, et le droit est supposé fonder la justice dans la société. Qu'est-ce que cela implique pour l'obligation?

Notre but n'est pas ici d'élaborer une philosophie nouvelle de l'Etat, mais de soulever certaines questions actuelles qui ont un rapport avec l'éthique de la justice, telle qu'elle nous apparaît dans ces pages. Certaines implications des évolutions récentes doivent en effet être gardées à l'esprit dans toute discussion éthique qui porte sur le rôle actuel et futur de l'Etat dans l'économie et la mise en place de la justice, et par conséquent sur la dimension «morale» de la responsabilité étatique. Ces développements sont essentiels pour éviter de retomber dans une élaboration nouvelle de thèses classiques sur l'Etat, qui s'appuyaient en leur temps sur leur propre expérience historique et donc sur une perception entièrement différente des pouvoirs et des devoirs de l'Etat. L'Etat est une réalité qui est essentiellement historique et qui ne peut être analysée utilement dans l'intemporalité. Il en est de même du rapport de l'Etat et de la justice. Il existe certes une idée de l'Etat, mais l'expérience historique nous meten présence d'Etats empiriques qui évoluent par la dynamique même de l'histoire, et quelles que puissent être les visions de Hegel sur l'Etat, la réalité est toujours différente. Car «ce que l'Etat prétend être de par son concept de la justice, à savoir "la réalité en acte de l"'idée morale objective' (Hegel, Philosophie du droit, chap. 257), aucune communauté empirique ni aucun Etat constitutionnel démocratique 
et social ne peut le revendiquer pour lui» ${ }^{16}$ Il faut donc toujours se référer à la situation particulière de l'Etat. Nous disons donc qu'un certain nombre de facteurs nouveaux doivent être mentionnés dans la discussion des «devoirs» de l'Etat face à l'évolution actuelle de la vie économique.

Le premier conceme ce que nous appelons la déterritorialisation des activitéséconomiques, conséquence directe du développement technologique et de la naissance d'un capitalisme aux dimensions du monde. Il n'est plus nécessaire, en effet, que la production, les échanges et la vie économique en général soient rattachés à un territoire particulier. Ceci a pour effet d'accélérer encore la séparation sociétale et institutionnelle de l'économie et de la politique, du domaine de l'action technologique et de l'action morale. Cette déterritorialisation a pour conséquence une séparation radicale des activités économiques d'une part, et du pouvoir réglementaire des Etats individuels de l'autre.

La dimension empirique, nous pourrions dire existentielle, des Etats, se définit en effet exclusivement par le territoire qu'ils dominent. Sans territoire sur lequel porte sa juridiction et sur lesquel le pouvoir étatique peut imposer souverainement ses lois, l'Etatn'existe pas. En tant que mondiale, l'économie échappe de plus en plus aux limites territoriales, ce qui transforme radicalement le rapport entre Etat et économie, et diminue considérablement le pouvoir réel des institutions politiques sur l'orientation et la dimension des activités économiques elles-mêmes. La force des lois est mise en question, et c'est auniveau des comportements individuels, en particulier par le développement de la contrainte morale inscrite dans l'éthique de la prospérité, que les comportements humains sont adaptés au fonctionnement général de la société économique. L'homo economicus remplace le citoyen. Les cultures et éthiques d'entreprises, nouveaux modes de comportement, identifiables aux grandes organisations économiques, se substituent peu à peu aux comportements nationaux stéréotypés que les règlements étatiques avaient pu imposer. L'éthique est donc appelée à l'aide pour faciliter une intériorisation des comportements que la loi étatique n'est plus capable d'imposer et de policer de l'extérieur. L'Etat n'étant plus capable de réglementer des activités qui dépassent ses frontières, les lois invisibles d'une économie toute puissante tendent à le remplacer. Mais cela a aussi pour conséquence que le rapport entre la loi et la moralité, souvent si essentiel pour le comportement individuel, ne joue plus de façon aussi stricte dans le monde de l'économie. Il devient de plus en plus difficile de moraliser la vie économique par le biais de la loi. L'acceptation quasi unanime des tendances actuelles vers une plus grande dérégulation en est la preuve. Allons-nous donc vers un monde régi par la loi du plus fort? Où serait alors la justice?

La deuxième évolution marquante est qu'il ne saurait y avoir de régulation sociale des marchés, - que l'existence de marchés capitalistes et de concurrrence

16 Otfried Höffe, op.cit., p. 364 
universalisée demande de toute façon - sans un pouvoir de légiférer qui est, par essence, un pouvoir étatique. C'est donc au moment où les Etats sont, pour ainsi dire, dépassés ou marginalisés par la mondialisation de l'économie, que l'on dénote la nécessité d'un nouveau pouvoir étatique pour garantir le bon fonctionnement de la concurrence et protéger l'individu, tant producteurentrepreneur que consommateur, contre le pouvoir gigantesque de l'économie elle-même. La conception de I'Etat, telle qu'elle pouvait exister au XIXe siècle ou dans la première partie du XXe, qui voyait en lui un instrument de contrôle des activités et initiatives de l'individu, contre laquelle le libéralisme contemporain a réagi avec force, se transforme en faveur d'une nouvelle idée de l'Etat qui fait de ce dernier un rempart juridique et moral contre une économie toute puissante qui tend à universaliser et imposer ses propres lois. L'Etat devient ainsi plus "protecteur», non pas parce qu'il tenterait de vouloir s'occuper de tout - tentation que l'on a suffisamment critiquée dans la mise en question de «l'Etat Providence» - mais parce qu'il est le dernier recours existant aujourd' hui contre l' immense machine technicoéconomico-capitaliste du monde.

Il y a donc de nouveaux devoirs de l'Etat. Or, si nous parlons de devoirs, nous introduisons une notion qui fait de l'Etat un sujet de responsabilité morale, du moins par rapport aux récentes évolutions de l'économie, et qui exige quelques clarifications..

L'éthique s'adresse avant tout à l'individu, au sujet. L'homme est avant tout sujet du devoir, et c'est à ce titre qu'il est sujet moral et personne responsable. Est-ce qu'en parlant de devoirs de l'Etat nous n'identifions pas ce dernier à un sujet moral, et ne faisons-nous pas de lui une personne? La chose devait paraître évidente dans l'histoire passée, quand le pouvoir de l'Etat s'identifiait à un Prince souverain. Les devoirs du Prince étaient les devoirs d'une personne individuellement responsable que la fonction différenciait des autres personnes, en faisant de lui un individu chargé de devoirs spéciaux. Mais ces devoirs du Prince n'étaient, formellement, pas différents des devoirs de n'importe quel autre individu remplissant une fonction particulière dans le cadre de sa vocation: devoirs du père de famille, du chef militaire, de n'importe quelle profession. L'Etatn'est plus, aujourd 'hui, incarné dans une personne. Notre époque, résultant de la démocratisation de la vie publique, nous fait vivre sous le régime de res publicae, de «choses" publiques qui, en tant que choses, ne peuvent être assimilées à des personnes humaines et à des sujets du devoir. Il est en effet difficile de penser que les grandes administrations anonymes auxquelles s'identifient les Etats contemporains puissent être porteuses de devoirs en tantqu' institutions étatiques. ${ }^{17}$

17 Certes les Etats sont sujets du droit international, mais celui-ci est un droit positif, relativement contraignant, et loin d'être toujours source morale de justice. Entre eux les rappports de force sont malheureusement plus déterminants que le rapport au droit. 
La deuxième difficulté vient de la libéralisation et de la sécularisation des sociétés modernes, ainsi que de la reconnaissance de la liberté de conscience comme un droit fondamental de l'homme, qui fait que l'Etat a du abandonner tout pouvoir d'intervention coercitive dans le domaine moral. Si l'Etat a maintenu des pouvoirs répressifs pour faire appliquer le droit positif, il ne peut prétendre détenir un quelconque pouvoir moral, au nom duquel il serait chargé d'imposer des comportements moraux particuliers à ses administrés. C'est pourquoi l'Etat est considéré comme moralement neutre. Il est a-moral, formellement dépourvu de toute responsabilité dans le domaine des jugements moraux. Même si l'on reconnaît qu'il doit intervenir parfois pour le maintien des moeurs sociales, ce n'est que dans le cadre du droit positif, comme par exemple dans le but de protéger des catégories sociales incapables de se défendre par elles-mêmes: protection des mineurs d'âge, des handicapés, etc.. L'Etat ne peut intervenir que sur ce qui est légal ou illégal, et non sur ce qui est moralement bon ou moralement mauvais. La liberté de conscience implique que l'Etat n'a plus de jugement moral a prononcer ni d'intervention à faire dans ce domaine. Le champ clos de la conscience morale individuelle et des comportements privés lui est interdit. Comment donc un être «a-moral», même s'il est une "chose publique», pourrait-il être sujet de devoirs?

L'Etat contemporain, démocratiquement fondé, trouve la légitimité de son pouvoir dans le support qu'il reçoit de ses citoyens qui sont, eux, par leur nature humaine, des sujets du devoir. C'est donc dans la nature de sujet des citoyens, dotés de liberté et de responsabilité, que se fonde l'existence et le pouvoir de l'Etat. Si les sujets disparaissaient en tant que sujets, s'ils perdaient leur responsabilité et leur liberté, il n'y aurait plus de légitimité pour fonder l'Etat, il n'y aurait donc plus d'Etat lui-même. La fonction première de l'Etat, dès lors, celle qui lui garantit son existence légitime, est de protéger la nature de sujet de ses citoyens, c'est-à-dire leur capacité d'êtres des individus porteurs de liberté et de responsabilité. C'est en ce sens que nous pouvons dire que cette fonction est un devoir, car il en va de l'existence même de l' institution étatique. Or c'est la condition indispensable pour que règne la justice, comme nous l'avons souligné. C'est à partir de ce premier devoir formel, protéger les individus qui, en tant que sujets, constituent l'Etat, que sont déduits tous les autres devoirs fonctionnels de l'institution étatique, à savoir la défense de la vie, de l'intégrité corporelle, de la liberté de conscience, d'éducation, de développement personnel, etc. L'Etat a des devoirs, et peut être considéré comme sujet de devoirs, parce qu'il n'existe que par les sujets qui le constituent. Il ne peut agir autrement, donc il doit.

Or nous avons justement reconnu que l'éthique de la prospérité, comme expression des fondements moraux d'un capitalisme mondial triomphant en cette fin de siècle, qui trouve dans le néo-libéralisme et les principes du "moins d'Etat» une philosophie politique adaptée au dynamisme de ses ambitions, peut mettre en danger la responsabilité de l'individu en faisant 
de lui un instrument, le simple objet utilitaire d'une croissance illimitée et irresponsable de la richesse. Tandis qu'il était admissible, au temps où la consommation pouvait être considérée comme la mesure du bonheur, que l'Etat soutienne le développement économique pour faire le bonheur de ses citoyens, il semble urgent de revoir le rapport entre Etat et économie au moment où, comme aujourd'hui, la finalité de l'économie a été séparée de la satisfaction des besoins et se concentre dans l'unique croissance de l'économie elle-même.

Il y a un devoir de l'Etat de défendre l'homme comme sujet, comme personne libre et responsable, contre l'objectivisation du sujet et la réducation de l'être humain à une fonction instrumentale que les succès de l'économie ont tendance à imposer. Or c'est un devoir de nature morale, car il concerne la nature morale de l'individu et la capacité de ce dernier à vivre comme être moral responsable. Même si, en tant qu'institution, l'Etat n'est pas responsable de la morale individuelle, et même s'il ne peut pas se substituer à l'individu dans les jugements de la morale en général, il «doit» faire tout ce qui est nécessaire pour que les individus soient en état d'être reponsables de leur propre morale individuelle.

L'Etat ne doit pas dire aux êtres humains ce qu'ils doivent faire, il doit les aider à être en état de décider par eux-mêmes ce qu'ils doivent faire. C'est en ce sens qu'il contribue au maintien de la Justice. Cela veut dire, face à l'éthique de la prospérité, qu'il y a une responsabilité de l'Etat à faire obstacle à tout ce qui, dans l'économie actuelle, pourrait mettre en danger l'individu comme sujet. Cela se manifeste déjà dans tout ce qui se dit ou s'écrit aujourd'hui au sujet de la défense des droits de l'homme et des libertés individuelles. L'Etat doit donc intervenir également quand ces droits sont mis en cause, non seulement quand ils le sont par l'intervention d'un pouvoir politique tyrannique sur un territoire donné, mais aussi devant la menace plus larvée et plus universelle d'une économie sans contraintes. Si la croissance de la richesse a pu être favorisée en libérant l'économie de contraintes étatiques qui en diminuaient l'efficacité, cette même richesse pourrait être destructrice de l'homme si sa croissance n'était pas contrôlée par le pouvoir coercitif des Etats, au service de la justice et de l'harmonie humaine. L'Etat producteur, celui qui a voulu assumer lui-même la fonction productrice de la société, a fait faillite, comme le montre l'échec du communisme. Mais il serait faux de croire, suite à cet échec, que l'économie doive et puisse vivre sans Etat, ${ }^{18}$ car c'est l'homme lui-même qui en deviendrait la victime. Il y a donc un devoir de protection de l'individu par l'Etat qu'il n'est pas possible d'ignorer.

Ce n'est pourtant pas dans ce devoir de l'Etat que peut être fondé le devoir moral de l'individu lui-même. C'est au contraire la morale individuelle,

18 Malgré les rêves de certains libertaires. Cf. David Friedman, Vers une société sans Etat 
celle qui constitue l'homme comme sujet du devoir, qui est elle-même la source et le fondement du devoir de l'Etat.

Or, c'est autour de la notion de justice que se manifeste l'obligation morale pour le gestionnaire d'Etat. La première exigence de ce devoir de justice est le respect de l'obligation des individus. Tous les individus sont une afin en sois, et doivent être traités comme tels; le sujet n'est jamais assimilable à un moyen. La deuxième est de trouver une manière juste de contrôler les conflits de devoirs des citoyens. Il ne s'agit pas de brimer, de nier la responsabilité morale des individus qui s'en inspirent, il s'agit de trouver l'équilibre nécessaire à une utilisation ujuste» du pouvoir légitime de répression. Le pouvoir d'Etat, par la justice, est celui qui le plus ouvertement recourt à l'imputation, la responsabilitế imposée de l'extérieur, qui est une des conséquences de la subjectivité essentielle de tous les actes moraux. De ce fait, une loi injuste est une loi qui mettrait l'individu dans la situation de ne pas être responsable de son acte. L'Etat ne peut jamais être un alibi d'a-moralité.

\section{Vers une éthique de la justice}

"On dit avec raison qu'une société n'a pas de conscience [morale]; mais une société d'individus dotés d'une conscience est une société avec une conscience. ${ }^{19}$ Les rapports de l'individu et de l'Etat sont généralement abordés du point de vue de la légalité, les questions éthiques étant d' habitude reléguées dans la sphère personnelle de la vie humaine. Mais la situation est différente dans la mesure où la société n'est plus simplement fondée sur un pouvoir souverain sans partage, celui d'un prince qui hérite d'un territoire qu'il gère, selon son libre plaisir ("L'Etat c'est moi»: Louis XIV), mais sur la participation et l'adhésion de tous ses membres, sujets responsables du devoir. La question de la justice est donc bien une question éthique, et non pas seulement légale.

Nous avons déjà affirmé que l'Etat, en raison de ses responsabilités dans la protection de l'individu, devenait un sujet de devoir, qu'il avait des obligations qui n'étaient pas seulement fondées dans l'affirmation et le maintien de son existence propre, mais dans la progression de la justice comme respect croissant des droits de l'homme. «Puisque les formes concrètes de la justice que l'on doit rendre positives ne se présentent nulle part à l'état achevé, le droit et l'Etat, notamment la jurisprudence, ont le devoir de les redécouvrir sans cesse ${ }^{20}{ }^{20}$ Cette obligation de l'Etat d'établir la justice est également un devoir du citoyen. Etat et citoyens sont dès lors solidaires dans leur devoir de promouvoir la justice.

19 Henry David Thoreau, La désobéissance civile.

20 Otfried Höffe, op. cit., p. 365 
Machiavel a parlé de l'Etat comme d'une institution dans laquelle l'incarnation et la poursuite d'intérêts particuliers pouvaient justifier tous les actes. La Raison d'Etat prétend ainsi à l'amoralité, et se situe par delà le bien et le mal. La tentation des Princes de jouir de leur pouvoir dans cet esprit a été permanente dans l'histoire. La première partie du XXe siècle a vu l'apogée d'idéologies politiques qui ont placé la légalité, fondée sur le pouvoir de l'Etat, au-dessus de la conscience des individus. Nationalisme et idéologie se sont conjugués pour annihiler la morale au nom d'un pouvoir arbitraire. Mais l'idéal de la démocratie ne permet plus de défendre intellectuellement de telles aberrations. Le jugement moral s'applique à l'action de l'Etat, en tant qu'acteur responsable de l'organisation de la vie humaine, au même titre qu'à tous les actes de l'individu. Avec la démocratie, la responsabilité morale des citoyens ne peut être mise entre parenthèses quand il s'agit des intérêts de l'Etat. Aucune circonstance ne peut dispenser de l'obligation; l'homme d'Etat, donc l'institution qu'il sert, comme le citoyen, est aussi sujet de l'obligation.

L'action principale de l'Etat est législative. Il établit le droit, l'applique, l'impose et punit ceux qui l'enfreignent. Mais le droit est à la fois justice et pouvoir. Tandis que le pouvoir par le droit demeure toujours limité, qu'il évolue avec le temps, s'adapte aux moeurs et peut être relativisé, la justice, elle, doit toujours être visée comme valeur supérieure, car il en va de la survie de l'homme dans le corps social. Si le droit limite la liberté, il ne doit jamais limiter la conscience morale, et en particulier la conscience de la justice.

L'éthique se présente donc, dans la primauté qu'elle doit avoir sur le droit, comme une limite où s' arrête le champ du pouvoir que le droit confère à l'Etat. Mais c'est aussi sur cette limite que la valeur morale de l'Etat et l'obligation du sujet se rencontrent dans l'idée de justice. L'obligation sociale, pour le citoyen, devient la recherche de ce qui est juste. Ainsi l'individu doit faire référence à son obligation personnelle pour découvrir "ce qui est juste» au-delà de ce qui est légal. La justice est donc l'objet d'une éthique de la personne beaucoup plus qu'une organisation de la société. Socialement, l'obligation s'épanouit en une éthique de la justice. Car, comme le soulignait déjà Aristote: «la justice contient toutes les autres vertus. $m^{21}$

Aristote poursuit son analyse et souligne trois idées princiales qui nourrissent sa réflexion sur la justice, celles d'égalité, de mesure commune et de réciprocité. La justice place donc le sujet dans un type de relation sociale qui implique ces trois éléments. François Ewald reprend cette réflexion dans son analyse de la justice sociale, et tente de montrer en quoi la compréhension contemporaine, épistémologique, de ces trois composantes peut contribuer à résoudre le problème qu'il soulève en ce qui concerne

21 Ethique de Nicomaque, Livre V, Chap. 1, 15 
l'Etat providence. ${ }^{2}$ Nous pensons que ces êléments peuvent nous permettre de comprendre ce que peut être une éthique de la justice construite à partir de notre réflexion sur l'obligation.

La notion d'égalité doit être prise dans une connotation morale, et non pas juridique. Il ne s'agit pas d'imposer une égalité abstraite là où des inégalités de fait sont inévitables, mais de reconnaître qu'en dépit des différences, l'attitude morale de l'agent par rapport à autrui doit viser à traiter tout le monde ( «tous les êtres de la même catégorie essentielle», selon Aristote) de la même façon. En d'autres termes, il n'y a pas de justice qui puisse s'appuyer sur des privilèges ou qui puisse légitimer une attitude discriminatoire envers certains sujets. L'égalité place tous les êtres humains dans la catégorie de l'autrui qui est, comme nous l'avons vu, la source de mon obligation. Cette considération morale de l'égalité rejoint ce que nous avions déjà souligné au sujet d'une distribution factice et exclusivement quantitative du bien-être, considérée comme contraignante et de ce fait amorale. Si l'essentiel qui est dû à chacun est le respect de sa nature de sujet et de sa vocation, l'égalité morale implique le respect de l'individualité la plus originale et la plus différente. L'égalité des conditions devient ainsi une égalité de destin, non d'un existant empirique.

La mesure commune nous ramène à ce que nous avons dit de la valeur et de la norme, qui permettent de rapprocher les choses dissemblables et de faire que les sujets soient engagés dans une poursuite de leur propre bonheur qui ait un sens pour tous. C'est avant tout ce rapport à l'humanité en général que chacun découvre dans son obligation personnelle et qui fait que l'individu puisse poursuivre le bonheur de toute l'humanité dans sa vocation personnelle. La valeur qu'il invente dans ses actes lui permet ainsi de participer à la construction d'une histoire commune.

La réciprocité est l'élément essentiel de ce que nous avons dit du partage, de cette relation essentiellement morale qui lie les sujets et qui fait que le don de soi soit mutuellement enrichissant pour tous. La justice est en effet la valeur qui favorise l'échange, qui va bien au-delà d'une simple redistribution égalitaire. Il y a plus dans un échange véritable, que dans la simple division quantitative d'une richesse matérielle, car l'échange permet au sujet de s'engager sans se perdre. «Si je te donne un oeuf et que tu me donnes un oeuf, nous avons chacun un oeuf; si je te donne une idée et que tu me donnes une idée, nous avons chacun deux idées». ${ }^{23} \mathrm{Il}$ en est de même dans le partage de la valeur, et la notion de réciprocité, dans l' idée de justice, en exprime parfaitement la dimension morale. Le sujet ne se perd pas dans le don qu'il fait de lui-même, comme il ne se nie pas dans son désir

22 Voir François Ewald, l'Etat providence, p. 552-554

${ }^{23}$ Voir Yoland Bresson, et Philippe Guillaume, Le Participat; réconcilier l'économique et le social, p. 38 
d'instaurer une justice universelle valable pour toute l'humanité. Cette réciprocité nécessaire n'exprime pas autre chose que la solidarité humaine.

C'est dans la perspective de cette éthique de la justice que l'individu participe activement à la construction de la société-sujet, cette société transformée par l'obligation morale qui favorise à la fois le déroulement humain de l'histoire et la justice partagée. Or, c'est dans tous les aspects de la vie sociale, religieux, moraux, politiques et naturellement économiques, que la participation des individus implique la contribution de chacun à ce que nous pourrions appeler une «prospérité générale dans la justice».

Cette participation est à la fois éthique et matérielle, individuelle et collective, faite d'exigence de justice et de compréhension des différences, d'accueil et de travail. L'obligation de travailler, et de contribuer ainsi à l'histoire matérielle et au progrès de l'humanité est aussi moral que la poursuite des idéaux les plus élevés. Il n'y a pas de véritable démocratie composée simplement d'ayant-droit, il faut des citoyens dotés de devoirs, et non pas simplement de droits et de privilèges. L'histoire de la justice ne s'écrit pas seulement avec le progrès des droits de l'homme, mais aussi avec la croissance d'une conscience de plus en plus intense des devoirs du citoyen. ${ }^{24}$

Nous pourrions le souligner en parlant du travail en termes de devoir et non pas seulement de droit. En faisant du travail un devoir, on évite de le concevoir comme une simple contribution contractuelle de l'homme à la prospérité matérielle, mais comme une participation du sujet à la vie morale. C'est prendre conscience que la valeur du travail ne vient pas tellement de sa contribution à l'augmentation globale des richesses que de la possibilité, pour l'individu, d'accomplir sa vocation au service de la société.

Comme nous avons découvert que l'obligation morale comprenait une obligation de se faire soi-même, il y a une obligation similaire qui doit être envisagée dans la contribution de chacun au bien-être de tous. Travailler est aussi une obligation envers autrui, fondement, comme nous l'avons vu, de l'idée d'obligation morale. L'idée de justice exige également cette participation.

Il faut envisager cette question à partir du rapport qui s'établit entre le travail et la propriété dans la construction du bien être individuel et social. L'idée de propriété découle de ce rapport. Tandis qu'il est impossible d'aliéner un individu de son travail, - l'action ne peut être dérobée au sujet agissant car elle lui est toujours propre - ce travail ne devient une richesse mesurable que dans l'accumulation de ses fruits. Certes, la propriété première est la capacité de l'individu d'agir, de faire quelque chose en tant que sujet, mais cette propriété, même si elle se manifeste dans une multitude d'actions individuelles, n'est pas cumulable; elle ne peut grandir par accumulation.

24 Un livre récent sur l'Europe souligne cette exigence: Joseph Rovan, Citoyen d'Europe; les devoirs avant les droits 
Ma compétence est ma première propriété, mais la simple utilisation de la compétence ne crée pas une richesse susceptible de croître, encore faut-il qu'elle le soit dans une action utile et partagée. La solidarité est l'espace dans lequel ma contribution au développement de tous s'inscrit concrètement. Voilà pourquoi l'éthique de la justice nous permet de comprendre que le bien-être matériel qui résulte de l'action est toujours légitime, car il peut être partagé. Et il n'est pas possible de partager moralement ce que l'on ne possède pas. La notion de justice doit aussi être introduite dans cette discussion.

Le danger de notre époque, nous l'avons vu, est de renverser le rapport du travail et de la richesse. Cette dernière ne peut devenir un but en ellemême, mais elle peut toujours contribuer à l'avancement de la justice.

Il y a, finalement, un devoir de participation de chacun au progrès. La prospérité peut être considérée, en effet, comme source de libération, donc devrait pouvoir contribuer au développement de la moralité dans la vie humaine et l'histoire. Travailler, en ce sens: c'est faire l'histoire. Les limites de l'éthique de la prospérité viennent non seulement de la conception exclusivement quantitative de la finalité qu'elle propose, mais aussi du domaine restreint de l'histoire humaine auquel elle se limite, car elle n'appelle pas l'être humain aux trois bonheurs possibles que nous avons mentionnés, le transcendant, l'immanent et l'englobant, mais à ce dernier uniquement. Orl'idée de justice, considérée comme valeur morale essentielle, ne peut s'imposer qu'en rétablissant les multiples dimensions de la vie humaine globale.

L'éthique de la justice s'ouvre donc sur la recherche d'une prospérité complète, celle qui concerne l'accumulation des vraies richesses humaines. 


\title{
Chapitre 9
}

\section{L'obligation morale et le progrès de l'humanité}

\author{
Lorsque Bach compose, il n'a pas \\ besoin de faire la guerre pour se créer \\ un destin.
}

Marcel Moreau Discours contre les entraves

Dès le début de ce travail, nous avons placé notre réflexion dans la perspective des développements historiques qui caractérisent notre civilisation, ceci dans le but de rattacher notre pensée aux longs efforts entrepris par la philosophie pour con tribuer à l'établissement de la moralité dans les sociétés humaines. Si, au lieu de limiter notre recherche historique aux constructions éthiques des philosophes, nous nous étions attaché aux descriptions des situations morales concrètes et aux témoignages des moralistes sur la moralité de leurs temps, nous aurions été frappé par le sentiment de crise permanente qui a du règner dans l'histoire morale de notre culture et aurions du constater que notre histoire n'a été qu'une longue suite de périodes de relâchement des moeurs et de décadence morale. Car c'est toujours à partir de la prise de conscience d'une crise morale que le moraliste semble s'éveiller à sa tâche et construire son oeuvre. Si l'histoire des idées morales se contentait de citer les introductions des ouvrages consultés, ses conclusions ne pourraient être que très pessimistes sur la capacité de notre civilisation à faire progresser et à améliorer les comportements humains. L'image qu'on en retire donne en effet l'impression que la morale n'a fait qu'empirer de génération en génération, et qu'à chaque étape de l'histoire des doctrines 
morales, on a vu descendre d'un cran le niveau moral de l'humanité. Or c'est justement dans ce pessimisme des moralistes que nous trouvons la preuve d'un progrès, car il manifeste justement combien la conscience morale est demeurée vive et active, et dans la plupart des cas de plus en plus critique, au point d'éveiller régulièrement l'attention des hommes sur le besoin d'inventer et de proposer de nouveaux idéaux et de nouvelles références aux yeux desquels les valeurs traditionnelles de la société pouvaient apparaître décadentes. A chaque étape de l'histoire, on constate donc que le paradigme des valeurs n'est plus satisfaisant, et que la décadence constatée est l'occasion de faire appel à de nouvelles exigences éthiques.

Voilà pourquoi l'accumulation des crises morales successives, telle qu'elle apparaît dans les écrits des moralistes, ne contredit pas l'idée du progrès moral, au contraire. Comme nous l'avons indiqué en analysant le besoin de dépassement de la contrainte morale, c'est au moment même où les valeurs admises semblent s'écrouler que l'invention morale peut le plus efficacement s'épanouir. Or c'est justement cette invention morale qui est à l'origine de ce que nous osons appeler le progrès moral. Nous souhaitons mentionner ici quelques conditions qui rendent ce progrès possible, dans la perspective de notre recherche en tenant compte du fait que c'est la prise de conscience de l'impasse dans laquelle conduit l'éthique de la prospérité qui est l'occasion de cette nouvelle invention.

Il est tout d'abord nécessaire de distinguer deux aspects dans la réalité morale:

- d'une part ce que nous appelons la «morale», ou l'éthique, c'est-à-dire l'élaboration rationnelle des valeurs morales et la réflexion sur le sens de l'action et la destinée morale de l'homme;

- d'autre part la "moralité», c'est-à-dire l'existence concrète, dans l'action accomplie, dans l'actualisation des personnes en devenir, des valeurs et des normes morales entrevues et choisies comme finalité morale.

D'une part les valeurs et la conscience morale; d'autre part la réalité ellemême, dans laquelle l'être se crée en se découvrant. Pour reprendre ici les deux approches de l'analyse à laquelle nous avons procédé dans ce travail, nous dirons que le premier aspect de l'histoire de la réalité morale sera de nature plus particulièrement conceptuelle, relatif à la pensée et à la culture, tandis que l'autre sera de nature plus existentielle, ressortissant à la vie concrète de l'homme.'

1 Cette distinction est reprise par de nombreux auteurs: Eugène Dupréel, par exemple, parle «du progrès de la conscience et du progrès de la vertu», Traité de morale, p. 449. On retrouve une idée similaire dans Ignace Lepp, La morale nouvelle, qui parle «du progrès moral et du progrès de la morale», p. 68 


\section{Le progrès des valeurs morales: l'effort de la conscience}

L'historique de l'obligation morale, présenté au Chapitre 3, peut être considéré comme un exemple de ce que peut devenir, dans ses grandes lignes, l'étude du progrès d'une idée morale particulière. L'obligation, en effet, s'est peu à peu dégagée, au cours de siècles d'analyse morale, comme l'un des concepts clés de la construction éthique. C'est par cette notion que des systèmes éthiques plus récents ont pu mettre en relief certains aspects de la vie morale qui seraient demeurés cachés sans le recours à l'idée d'obligation. De ce point de vue, il est donc possible de dire que l'obligation représente un progrès dans l'étude et la compréhension de la condition morale de l'homme.

Mais avant d'analyser les valeurs proprement dites, et montrer comment elles ont progressé dans l'histoire, il est nécessaire de préciser ce que nous entendons par progrès, et ce que cette notion implique dans notre propos.

Dire qu'il y a un progrès dans l'histoire signifie que nous portons, sur l'histoire elle-même, un jugement de valeur a priori, par lequel nous nous autorisons de juger à l'avance le mouvement historique que nous nous proposons de découvrir. Car la notion de progrès est avant tout une notion axiologique. Parler de progrès, c'est impliquer que l'histoire a un sens, qu'elle poursuit un cheminement avec une raison d'être, qu'elle s'oriente vers une fin qui nous apparaît comme bonne, ou du moins comme meilleure. Nous sommes donc bien conscient qu'en proposant l'idée de progrès nous affirmons à l'avance ce que nous allons tenter de prouver par la suite. Il faut donc préalablement croire au progrès pour être capable de le découvrir ensuite dans l'observation de l'histoire. Mais cette idée s'expose aux critiques et aux preuves contraires que peut avancerl'observation empirique.

Il n'y a, pratiquement, du point de vue de l'empirisme le plus pur, qu'un seul progrès possible qu'il ne soit pas possible de rejeter comme une pétition de principe, c'est celui qui s'appuie sur des réalités quantifiables. Toutes les théories de la croissance économique qui ont caractérisé cette deuxième moitié du XXe siècle sont fondées sur cette certitude. C'est, nous l'avons vu, sa dimension quantifiable qui rend l'éthique de la prospérité si puissante et si convaincante. Si le critère du progrès se situe dans le "plus», chaque étape mesurable contribue à prouver l'existence d'un progrès des sociétés et de l'homme.

Il en est de même de la performance des technologies dans ce siècle où la croissance asymptotique des performances techniques ne peut être interrompue et semble enfermer les sociétés techniciennes dans un engrenage irrépressible. Trop souvent, en effet, c'est en faisant appel à davantage de technologie que l'on tente de résoudre les problèmes causés par la technologie elle-même. Mais à force d'en dépendre, l'humanité se trouve dans la situation de ne plus pouvoir s'en sortir, comme un être intoxiqué. Il existe 
donc une forme de econdamnation au progrès" qui constitue le piège final de l'homme contre lequel nous avons proposé de réagir. Mais peut-on dire qu'il existe un réel progrès dans ce cas? Les soi-disant progrès quantifiables sont donc sans limites. Au nom de quelle norme et de quel critère l'homme sera-t-il capable de les évaluer?

Le domaine moral, lui, ne se laisse pas enfermer dans un tel déterminisme. Nous croyons qu'il faut prendre un risque de la pensée pour parler de progrès moral, un risque qui consiste à inventer, et non pas à prouver, la valeur du progrès lui-même. Il y a dans cette démarche comme un acte de foi, ou d'invention volontaire, qui consiste à s'exprimer en faveur d'une valorisation de l'histoire et à procéder à une reconstruction de l'histoire au nom des valeurs. Or, en raison de ce que nous avons affirmé de la valeur et de sa relation essentielle au devenir de la personne, le progrès doit être considéré comme une personnalisation continue de l'histoire. Nous avons indiqué que cette personnalisation, ou ouverture au sujet, se manifestait déjà dans les sociétés religieuses et politiques, et que notre but était de la faire intervenir également dans le domaine de la vie économique et technique.

Le progrès ne se manifeste cependant pas dans les choses elles-mêmes, mais dans le regard que l'être humain porte sur les choses. Il est une des valeurs que l'homme apporte au monde. Croire au progrès, c'est accepter de refaire l'histoire au nom de notre foi dans la personnalisation de la vie et des comportements humains. En d'autres mots, notre croyance au progrès est une façon de refaire l'histoire en fonction de ce que nous pensons aujourd'hui du devenir de l'homme. Si, à chaque étape du développement de la conscience morale, l'histoire est ainsi reconstruite à partir des nouvelles visions subjectives qui naissent par l'obligation, il y a donc réellement un progrès, celui qui s'incarne dans chacune des réinterprétations du passé.

En nous plaçant d'un point de vue purement scientifique, il faudrait que la finalité de l'évolution de l'humanité soit inscrite dans les choses, et déduisible à partir de la réalité soumise à l'observation. La finalité de l'espèce doit être ainsi contenue dans les espèces actuelles, comme toute la vie humaine est inscrite dans les gènes qui la déterminent, ceci depuis les premières cellules vivantes qui se sont développées au fond des océans. Mais si nous considérons l'évolution et la finalité de l'humanité d'un point de vue moral, elles doivent résulter non pas de ce qui est déjà donné, mais d'une série d'inventions humaines. Vers elles doivent conduire les efforts de valorisation de l'homme et du monde qui résultent de longs siècles d'interrogation et de prise de conscience de l'homme par lui-même.

C'est la nature même de l'invention de ne pouvoir être préétablie. C'est pourquoi la finalité morale de l'histoire se présente toujours comme un idéal. "Quoi qu'on fasse», écrit Bergson, «il faudra toujours revenir à la conception de créateurs moraux, qui se représentent, par la pensée, une nouvelle atmosphère sociale, un milieu dans lequel il ferait meilleur vivre, je veux dire une société telle que, si les hommes en faisaient l'expérience, 
ils ne voudraient pas revenir à leur ancien état. Ainsi seulement se définira le progrès moral». ${ }^{2}$

Le progrès moral n'est donc pas à déduire de l'histoire, mais à inventer par rapport à un idéal, au nom duquel l'histoire va devoir être réinterprétée, réinventée, en découvrant sa lente marche à travers les démarches répétées, les tentatives renouvelées, les échecs dépassés, les obstales vaincus. $\mathrm{La}$ croyance au progrès de l'humanité est une démarche qui consiste à voir comment, à chaque étape reconnaissable de notre héritage, des inventeurs moraux ont pu arracher du ciel une partie de l'idéal qu'ils y entrevoyaient et l'ont placée sur le cheminement des hommes, comme un témoignage et une garantie concrète de l'épanouissement final.

Nous avons dans cette perspective la conviction que la conscience des valeurs morales a connu un progrès, et ceci pour plusieurs raisons.

D'abord, nous constatons que, d'une façon générale, la pensée humaine progresse de plus en plus vers des modes réflexifs, par l'affirmation du sujet (à partir du cogito cartésien) et la connaissance de la personne (depuis Kant, la phénoménologie et l'existentialisme). C'est à nos yeux un progrès puisque la réflexion morale a permis de prendre plus nettement conscience de la nature de l'homme comme «subjectivité», mode irremplaçable d'être, pour l'homme, dans le monde et pour le monde. Mais puisque le progrès moral n'est pas inscrit dans les choses, il n'est jamais garanti, et la pensée réflexive pourrait perdre ses acquis si, en transformant trop facilement ses découvertes en système, elle oubliait le choc du "connais-toi toi-même» socratique. L'ironie socratique, cette façon de faire naître l'individu à luimême, nous apparaît aujourd'hui, existentiellement, comme un instrument privilégié de la possibilité du progrès moral car sans elle il y a toujours un danger de dissolution du sujet dans l'extériorité et une tentation mortelle de substituer les systèmes à la personne. C'est contre une telle systématisation de la subjectivité, comme elle a été pratiquée en particulier dans les philosophies de l'idéalisme allemand, qu'est née par exemple la réaction existentialiste d'un Kierkegaard. C'est dans cette perspective que l'ironie socratique nous apparaît encore comme un instrument utile pour compenser la dissolution du sujet dans la performance, l'un des dangers de l'éthique de la prospérité.

Certes, nous ne nions pas qu'il soit possible de construire un système cohérent à partir de la découverte de la subjectivité, - tant de philosophies l'ont fait - mais si cette subjectivité n'est pas constamment affirmée comme «existence concrète», répondant à l'injonction de Delphes et appelée à la personne par l'obligation, ces systèmes nous semblent inutiles pour la morale. "L'ironie socratique sera toujours l'essence de l'éducation morale, parce qu'elle éveille un besoin, en laissant à celui qui le ressent le soin de

2 Henri Bergson, Les deux sources..., p. 80 
résoudre la contradiction ${ }^{3}$. Il n'est donc pas difficile, à partir de cette expérience, de retrouver le cheminement du progrès des valeurs morales dans l'histoire jusqu'à la contribution de l'existentialisme contemporain. Il y a progrès quand la pensée fait appel à l'éveil de la conscience et à l'invention morale, - jusqu'au risque du Mal, comme nous l'avons vu avec GOETZ - contre l'emprise des systématisations cohérentes du passé. La rigueur philosophique n'est source de progrès que dans la mesure où elle contribue à l'épanouissement du moi qui, à partir du cogito, est entraîné à la découverte de son être agissant.

Deuxièmement, la morale est en progrès en raison même des transformations que la situation de l'homme a subies à travers l'évolution des sociétés ellesmêmes. Nous avons indiqué comment le conflit des sociétés pouvait conduire au dépassement de la contrainte morale, et favoriser l'invention de la conscience. Avec la complexité progressive de la vie sociale, - accélérée dans l'époque moderne par l'industrialisation, le progrès technologique, la mondialisation de l'économie et des échanges, la démocratisation de la vie politique, ainsi que la déterritorialisation de la vie économique et individuelle - la révélation de la personne comme valeur suprême et sa responsabilisation en sont favorisées. Quand l'individu est engagé dans plusieurs structures sociales et que les sphères de son activité se multiplient, il est forcé de se retrouver, face à sa propre existence et à sa responsabilité; il lui est alors possible de tout gagner ou de tout perdre.

Ceci avait déjà été souligné par une analyse plus ancienne de Dupréel, par exemple, qui, dans une critique de la théorie de la conscience collective selon Durkheim, montre que le développement de la société n'entraîne pas une concentration de cette conscience collective sur elle-même, mais bien plutôt une sorte d'éparpillement de la force collective, dans une foule de sociétés différentes, dans un nombre plus considérable de groupes. C'est ainsi que les conflits de normes sociales et de valeurs morales se multiplient et que, du point de vue de la personnalisation, le "progrès» de la civilisation marque, en fait, "le crépuscule de la conscience collective». ${ }^{4}$

Ce progrès doit être vu du point de vue moral, et non pas seulement psychologique. On sait en effet que la collectivisation des sociétés contemporaines, ou la naissance de la civilisation de masse, qu'elle soit le résultat d'un pouvoir idéologique totalitaire, comme en Chine, ou celui de l'expansion du marché et de la consommation, comme dans la majeure partie des pays capitalistes, ou encore de l'urbanisation intensive, peut entraîner un déracinement social et culturel et une annihilation de la personne dans la solitude morale et l'isolement. ${ }^{5} \mathrm{Ce}$ phénomène se retrouve dans le processus de «déliance», qui enlève à l'individu tous ses points de

\footnotetext{
3 Rene Le Senne, Le devoir, p. 147

4 Dupréel, op. cit., p. 414

5 Voir à ce sujet, David Riesman, La foule solitaire
} 
repère, y compris ses références comportementales. ${ }^{6}$ Mais ce danger social d'annihilation de la personne n'est-il pas lui-même un conflit nouveau, une situation morale nouvelle qui présente une nouvelle forme d'obligation et l'occasion d'un nouveau dépassement?

Il en est de même de la recrudescence des sentiments nationalistes qui marque le démantèlement actuel des grands ensembles politiques et qui entraîne apparemment un retour de la conscience collective sur elle-même. Il ne faut pas considérer ce phénomène comme une régression irrésistible des sociétés, mais comme la réaction normale des individus devant une ouverture trop subite de la société vers des formes multi-culturelles de valeurs et de moeurs. Car cette ouverture peut elle-même être l'occasion d'offrir à la créativité morale de nouvelles inspirations. Les idées de Karl Popper sur la «société ouverte», au sein de laquelle le progrès humain devient possible dans la multiplicité des expressions sociales et politiques, corroborent notre point de vue.

La rupture de l'homogénéité des groupes géographiques et historiques à laquelle nous assistons aujourd'hui dans le monde, entraîne des conflits incessants qui devraient permettre à l'homme, dans la mesure où il est disposé à répondre à l'appel de l'obligation, d'accèder à une prise de conscience à la fois plus grande et plus profonde de son individualité et de sa personne, ainsi que de sa solidarité universelle par l'éthique de la justice. C'est pourquoi nous pensons que les dangers de la civilisation contemporaine, comme on les dénomme souvent, ne sont en fait des dangers que lorsqu'on les compare à la situation passée qui se désintègre sous nos yeux. Il ne le sont nullement si on les considère comme des occasions d'ouverture vers un nouvel idéal. Le pessimisme culturel et moral n'existe que pour ceux qui ne croient pas à l'invention morale et au pouvoir de l'obligation. Les chemins qui conduisent à la personnalisation se renouvellent sans cesse, et ceux de demain ne seront pas ceux que l'humanité a empruntés hier.

Mais cela ne veut pas dire qu'il n'y ait plus de progrès possible vers davantage de personnalisation. L'obligation morale nous fait un devoir de penser à l'homme d'abord, à ce qu'il est et ce qu'il peut devenir, et non à la moralité qui a pu résulter des idéaux du passé. La civilisation nouvelle qui émerge en cette fin de siècle va entraîner le renversement de nombreuses valeurs, mais elle n'enlève rien à la force de l'obligation comme source de l'invention des valeurs nouvelles. Au contraire, puisque les valeurs morales doivent être remises en question, l'importance de l'obligation ne fait que se renforcer.

La remise en question de nombreuses valeurs morales traditionnelles n'est pas en elle-même une source d'inquiétude si l'on sait observer la

6 Ce phénomène a été récemment analysé par Marcel Bolle de Bal, La tentation communautaire, Les paradoxes de la reliance et de la contre-culture

7 Karl Popper, The Open Society and its Ennemies 
nature même de l'évolution qui nous force à les remettre en cause. Or nous croyons qu'il y $a$, dans ce domaine, un progrès certain vers une plus grande «spiritualisation» de l'interrogation morale. Que l'on considère, par exemple, les innombrables tabous et commandements de toute nature qui n'avaient pour but que de contrôler les comportements hygiéniques de la société. Les habitudes alimentaires et les comportements sexuels ont longtemps été codifiés et "moralisés", même par la loi religieuse, pour de telles raisons. Les interdictions marquées d'une empreinte religieuse, souvent accompagnées de menace de sanction dans l'au-delà, ont souvent renforcé le caractère obligatoire de ces normes qui n'avaient d'autre origine que le souci du bienêtre physique et du contrôle de la santé. La plupart de ces interdictions, formulées autrefois dans les décalogues moraux des sociétés, sont devenues aujourd'hui de simples conseils de prudence, sans consonance morale aucune. Certes il est évident que certains esprits souhaiteraient brandir à nouveau la menace divine pour conjurer le fléau du SIDA, mais l'esprit du temps ne correspond déjà plus à de telles incantations.

Dans ce domaine, nous croyons que la science et son pouvoir demystificateur ont grandement contribué au progrès de ce que nous appelons la *spiritualisation" des valeurs morales, même si ce rôle n'a jamais fait partie des revendications du monde scientifique, au contraire. En ramenant certaines valeurs morales d'autrefois au niveau des techniques de l'activité humaine, en révélant certains processus inconscients capables d'expliquer ces conduites qui venaient de l'homme mais qui n'étaient pas proprement humaines, en faisant découvrir à l'homme que ce qu'il prenait souvent comme un devoir n'était qu'une simple habitude culturelle ou un conseil d'hygiène, la science a ainsi conduit l'homme à s'attacher plus strictement à ce qu'il y avait d'authentiquement moral dans son action, et ainsi à spiritualiser davantage sa réflexion morale. Dans le conflit de valeurs que les progrès de la science (qui ne sont pas tous quantitatifs, d'ailleurs) ont directement causé dans les Weltanschauungen contemporaines, l'homme a été pour ainsi dire forcé de purifier, pour ainsi dire, ses conceptions morales et à les imprégner davantage de la valeur propre de l'esprit.

C'est ainsi qu'il a été possible de découvrir, par de nouvelles valeurs morales, que le domaine de l'esprit pouvait également être ouvert et conquis, comme l'être de la personne, par l'invention morale. Cette ouverture vers une conscience morale plus spirituelle, est peut-être la vraie conquête de l'histoire humaine. L'esprit est ainsi placé au sommet de la hiérarchie des valeurs de l'homme. Que l'on voit en lui la langue de feu de l'Esprit éternel, descendu de la transcendance divine pour transformer l'homme à son image, ou qu'on l'imagine comme résultant de la complexité croissante de la vie, en particulier dans le cerveau humain, couronnant ainsi une évolution naturelle de la matière, il n'en demeure pas moins que l'humanité est orientée vers une reconnaissance de cet esprit comme le sommet de la hiérarchie des valeurs et la finalité de son devenir. Chaque invention qui s'en rapproche, chaque pas de l'humanité vers une affirmation plus claire 
de la suprématie de l'esprit dans le choix des valeurs morales constitue donc un progrès. Le saut, - mais faut-il parler d'un saut, comme s'il y avait une différence de nature? - disons le passage, (mais il faut reconnaître qu'il n'est ni linéaire ni quantitatif, mais entièrement axiologique) de la subjectivité à la spiritualité de l'homme, est une des formes du progrès des valeurs morales. L'esprit devient ainsi une nouvelle étape de la conquête de soi. La science a contribué très fortement à cette prise de conscience, par son refus même d'introduire la notion de valeur dans ses méthodes, conduisant ainsi la réflexion à lui donner une place toujours plus grande dans la vie morale. Il y a donc progrès par spiritualisation des valeurs.

Reconnaissons finalement que le progrès des valeurs est un phénomène de culture, et par conséquent d'éducation. Les valeurs ne sont pas seulement affirmées dans l'action isolée de quelques sages ou héros engagés dans I'invention morale, mais s'inscrivent dans l'héritage culturel et se présentent à nous sous la forme d'idées, de normes, de modèles, d'interrogations qui stimulent notre propre conscience par la connaissance que nous en acquérons. C'est ainsi que se constitue notre conscience commune. Parler ici de "conscience commune» ne signifie pas que nous acceptons ce que nous avons rejeté plus haut sous le vocable de conscience collective; la conscience commune est celle que des sujets partagent solidairement, ce n'est pas une force inconsciente qui s'impose à eux et qu'ils subissent. Il faut d'abord rejeter toute forme de conscience collective irréfléchie et non responsable, pour parvenir au stade de la conscience commune. La reconnaissance de ce fait a inspiré ce besoin d'analyse historique dans laquelle nous avons voulu enraciner notre propre recherche. La morale progresse ainsi par le truchement de la culture et de l'héritage intellectuel. Les grandes ruptures morales de l'histoire de l'humanité ne sont donc pas à refaire éternellement. Moïse, Socrate, Budha, le Christ, Mohamed, Gandhi, ... et tant d'autres, nous transmettent leur message et leur invention morale dans la mesure où nous nous laissons imprégner de notre propre culture. Il existe en effet un dépôt de valeurs qui nourrit notre mémoire et qui permet de ne pas partir de zéro dans la conquête de nous-mêmes.

Certes, comme nous l'avons souligné, l'éducation morale par l'héritage culturel a des limites, car la connaissance des valeurs ne suffit pas à assurer leur actualisation dans la réalité morale, et ce n'est pas parce que nous pouvons acquérir la connaissance des valeurs qui ont été accumulées dans notre histoire que la moralité connaîtra un progrès équivalent. Il n'empêche néanmoins que ce dépôt de valeurs ne peut laisser indifférents ceux qui en prennent connaissance. Certes nous ne croyons pas, à l'instar de Socrate, que la simple connaissance du Bien garantit la rectitude morale de l'action, il y faut encore l'intervention de la volonté. Notre conviction est que la connaissance des valeurs du passé nous aide à prendre conscience de nousmêmes, de notre situation historique dans le déroulement du progrès des valeurs. Or, comme nous l'avons démontré, se connaître, pour l'homme, c'est toujours se transformer. Voilà pourquoi le progrès est possible; car 
plus il y aura de valeurs affirmées et connues, plus cette connaissance pénétrera la conscience des êtres humains par la transmission de la culture, plus facilement ceux-ci seront tentés de s'appuyer sur leur passé pour le dépasser dans une nouvelle création de valeurs. Pour que celle-ci soit une authentique création, d'ailleurs, il faut que la conscience morale ne cherche pas à faire revivre les valeurs telles qu'elles se sont présentées, mais à les assumer dans la perspective de l'idéal vers lequel elles tendent. C'est dans cette mesure, seulement, que l'accumulation culturelle des valeurs morales peut conduire à un réel progrès moral.

\section{Le progrès de la moralité: créativité de l'individu}

La connaissance des valeurs morales ne produit pas automatiquement la moralité. Comme le montre l'exemple de GOETZ: puisque Dieu a déjà fait le bien, il n'y aurait plus rien à y ajouter. Ce n'est toutefois pas la connaissance seule qui fonde sa décision morale. Comme tout homme, il doit tirer de lui-même les raisons de son vouloir. Des valeurs qui apparaîtraient entièrementétrangères à son propre devenir et sa vocation, $n$ ' auraient aucun pouvoir sur sa volonté et ne pourraient aucunement nourrir son obligation. C'est pourquoi les valeurs morales, comme la justice, ne sont jamais définitivement acquises, mais demeurent précaires, incertaines, inachevées, à la limite douteuses. Si le bien se présente comme une réelle certitude, il n'appelle pas à l'invention. L'homme est alors tenté de le rejeter. «Si le bien est déjà fait», clame le héros de Sartre, «alors je ferai le mal». L'accumulation des actions bonnes ne suit donc pas une logique historique similaire à celle des valeurs.

Voilà pourquoi nous pensons que tandis qu'il est possible de parler d'un progrès de la conscience et des valeurs morales dans l'histoire, il n'est pas possible de parler, de la même façon, d'un progrès parallèle de la moralité.

Cette dernière, en effet, ne peut pas être identifiée à l'actualisation d'une société historique, semblable à celle que tente d'établir la contrainte sociale. Elle est d'abord le devenir de l'être-de-la-personne dans I'unicité de l'élan qui le conduit à la conquête de soi-même. L'intelligence peut certes s'appuyer sur la connaissance des valeurs héritées des générations précédentes, et puiser dans la mémoire morale de la société les valeurs susceptibles d'orienter une action, ou de la juger. Mais c'est toujours la volonté, dans un acte unique, en réponse à une obligation hic et nunc, qui décide de l'actualisation réelle de ces valeurs ou qui rend possible la création de nouvelles valeurs. Ceci se produit dans l'intimité indivisible du devenir de la personne de l'agent, dans une relation unique avec les personnes d'autrui qui sont, dans cette situation précise, les co-auteurs de la moralité. Alors que la morale s'élabore peu à peu par cette lente accumulation de valeurs qui constitue la culture morale d'une civilisation, et reste fidèle à elle-même à 
travers les vicissitudes de l'histoire, la moralité elle, est toujours en situation, c'est-à-dire qu'elle doit toujours renaître dans le présent, concrètement incarnée dans l'existence des sujets qui la produisent. Voilà pourquoi la moralité n'a pas d'histoire.

La moralité dont je suis responsable ne survit pas à ma mort, car je suis essentiel à son existence. La moralité qu'autrui construit avec moi ne survit pas au départ d'autrui, car autrui est essentiel à son existence. La moralité sociale, ou la justice, qui résulte de l'action "partagée» des membres du groupe ne survit pas à l'histoire de leur intervention. La moralité est un éternel recommencement.

Il n'est dès lors possible de parler du progrès de la moralité que dans le cadre du développement de la personne, ou dans celui d'une communauté de personnes, identifiée dans l'espace et dans le temps. Comme la moralité ne peut exister en dehors d'une situation précise, c'est seulement par les personnes et dans les réalités qui composent cette situation qu'elle peut être saisie et comprise. Son progrès, ou son recul, ne peut être mesuré que par rapport à elles.

Mais si le progrès de la moralité est limité dans l'espace et le temps il peut, par contre, être illimité en intensité et en valeur pour les personnes qui y ont leur part. Il n'y a pas de limites à l'intensité et à la qualité du Bien. Mais, d'autre part, comme la moralité n'existe que par des personnes, il n'est pas possible de considérer la moralité manifeste dans une situation précise comme une moralité acquise une fois pour toutes. Valeur suprême de l'existence morale, la moralité est suprêmement précaire. On ne construit pas la moralité comme on construit les cathédrales. Chaque génération, chaque groupe, chaque communauté de personnes, chaque personne individuelle, doit sans cesse en poser à nouveau la première pierre.

On comprend donc pourquoi la moralité est toujours invention, création renouvelée, car il faut à chaque instant, dans chaque situation particulière, l'actualiser à nouveau par ses actes. Prenons un exemple. Si l'on peut dire que la communauté des frères que Saint François d'Assise avait réunis autour de lui vivait une moralité très intense, qu'elle avait même progressé depuis les premiers jours où le fondateur les avait entraînés dans cette aventure morale et religieuse, cette moralité n'a rien garanti pour l'avenir, ni pour l'ordre des frères mendiants, ni pour Assise, ni pour l'Eglise, ni pour l'Europe.

On n'hérite pas de la moralité comme on hérite des valeurs.

Il faut, à chaque instant, la produire à nouveau. C'est ainsi que les plus belles communautés morales, aussi idéalistes qu'elles aient pu être, ont souvent dégénéré dans le désarroi, l'inconsistance et la déchéance morale 
après la mort de leurs fondateurs ou des personnalités morales qui les ont animés. Il ne suffit pas de connaitre les règles, les valeurs ou les normes pour maintenir la vie morale à un haut niveau. If faut toujours la reconstruire.

C'est pourquoi, aussi, la moralité ne se répète pas. Elle est irremplaçable et inimitable. Il est inutile de vouloir tenter de rétablir certaines conditions de vie etcertaines circonstances d'une période révolue ou d'une communauté dans lesquelles la moralité était vivante et exemplaire, pour retrouver, comme parautomatisme, une actualisation similaire de moralité. Les personnes ne se renouvellent pas, aussi la moralité qui est fondée sur elles, et non sur les circonstances, ne peut têtre reconstruite artificiellement. Le personnalisme éthique conduit nécessairement à une morale de situation, donc changeante, car les situations ne se renouvellent jamais puisqu' elles impliquent toujours d'autres personnes.

C'est pourquoi la moralité est toujours au devant de nous, elle est toujours à créer. Nous pouvons le constater, par exemple, dans une foule de mouvements de réforme qui ont existé dans l'histoire chrétienne, qui ont tenté de reconstituer un idéal moral à l'image de ce qu'ils pensaient être la primitive église, et qui ont conduit àl'échec, et parfois même à l'immoralité. Leur erreur a été de vouloir vivre à la place d'autrui, de reconstituer la vie d'autrui, plutôt que de répondre à une obligation qui ne pouvait être que leur propre obligation. La rectitude morale est toujours individuelle, jamais transmissible et jamais imitable.

Cette confusion existe aussi chez les philosophes. Illustrons ce problème par une citation de Guyau, que nous avons analysé plus haut dans ce livre. "J'ai peut-être en moi,» écrit-il, «une énergie de volonté qui, il y a une quinzaine de siècles, m'eût transformé en martyr; de nos jours, je reste bon gré mal gré un homme ordinaire, faute de bourreaux. Que notre siècle, en somme, est pauvre en vrai mérite, quelle décadence aux yeux d'un partisan de la liberté et de la moralité absolue! Si le monde n'a pour but que de nous poser le probleme moral, il faut convenir que la barbarie le posait avec bien plus de force que la civilisation. Nous sommes trop heureux, aujourd'hui, pour être profondément moraux».

Qui n'a pas, comme Guyau semble l'espérer, été tenté de trouver dans des circonstances extraordinaires l'occasion d'un acte d'héroïsme capable de satisfaire le besoin subjectif de moralité? Mais c'est une erreur de croire que la moralité est définie en elle-même, comme quelque chose d'immuable, une sorte de vie héroïque extraordinaire au nom de laquelle l'agent moral devrait être prêt au martyre à chaque instant. L'erreur est de ne pas voir que la moralité n'est pas celle que nous demande le monde, mais celle que nous devons inventer. Car l'obligation n'est pas dans un contenu moral, dans ce que le monde ou la société nous demande, mais seulement dans notre propre

8 Jean-Marie Guyau, Esquisse d'une morale sans obligation ni sanction, p. 17 
attitude créatrice. Croire ainsi que la civilisation nous rend trop heureux pour être moraux, c'est imaginer l'obligation comme une contrainte de la nécessité, comme si l'héroïsme moral exigeait que l'humanité soit en guerre permanente pour susciter les valeurs morales. L'obligation morale ne se trouve pas dans la contrainte causée par le malheur. Le véritable héroïsme moral, qui n'est pas étranger d'ailleurs à la vertu de courage, se trouve dans l'invention morale, la création des valeurs, dans la solitude du choix et de l'action où le héros moral renverse le conformisme en le dépassant. C'est dans cette perspective qu'il faut envisager le progrès de la moralité. Celle que le sujet construit lui-même, en répondant à sa propre obligation dans chacun des instants de sa vie.

\section{La croissance de l'humain: l'homme responsable}

Il n'y a donc pas de progrès historique de la moralité. Il ne peut y avoir que des progrès personnels dans la moralité.

Certes, il demeure vrai que le contenu de valeur de la moralité sera inspiré de celui que la personne trouve dans sa culture et son temps, et complété par celui qu'il crée lui-même. La moralité du barbare, dans laquelle Guyau voyait une occasion d'héroïsme, n'est pas celle qui peut inspirer notre époque, pas plus qu'elle ne pouvait être utile à Socrate ou aux Stoïques. Mais cette différence n'est qu'une différence de contenu. Essayer de voir dans les circonstances extérieures de la vie l'occasion de faire grandir l'homme c'est en fait le soumettre au déterminisme du monde et de la société-objet. Or ce qui compte, dans l'obligation, c'est la recherche de I'homme lui-même, sa personne, sa valeur irremplaçable, et ceci à travers toutes les manifestations différentes de la moralité. La moralité ne peut pas être limitée à son contenu et aux conditions de sa réalisation; elle ne peut exister que dans sa forme. Or la forme de la moralité, elle, est toujours la même: c'est l'engagement de la personne elle-même dans son propre devenir. C'est là que se trouve l'obligation, et c'est pourquoi le progrès de la moralité est toujours personnel.

Nous devons garder cette distinction à l'esprit quand nous parlons du progrès moral, et reconnaître qu'il n'y aurait pas de progrès réel sans l'intervention de la personne. C'est en ce sens que nous pensons que le progrès moral passe par une croissance de l'humain, c'est-à-dire de l'être sujet du devoir dans tous les actes de la vie. Mais en faisant appel à l'idée de progrès dans ce sens nous proposons une valeur qui, par son sens même, incite obligatoirement à l'invention. La croissance de l'humain met donc le poids du devenir sur la responsabilité créatrice de l'individu. Le progrès n'est donc pas étranger à l'obligation morale elle-même. Il lui est même essentiel puisque cette obligation n'a pas d'autre finalité que le devenir de l'homme. 
La question du progrès moral se présente donc à l'individu comme une obligation, car elle ne peut trouver de réponse que dans une création existentielle. Le progrès moral devient ainsi lui-même une valeur morale et demande par là-même à exister. Il est le contenu authentique d'une obligation.

C'est dans l'intimité d'une obligation non transmissible que l'hommesujet doit trouver les réponses à toutes ses questions. C'est au sein de la responsabilité totale qui lui incombe qu'il peut s'ouvrir aux vraies dimensions de l'humanité qu'il porte dans son existence. Face à l'enrichissement progressif de nos sociétés sur-performantes, devant la pletthore de biens et de moyens que lui offre une économic toute puissante, il doit trouver en luimême le sens des choix et des actions par lesquels il marquera le champ spécifique de sa moralité. Le recours à une obligation comme source de sa propre existence en tant qu'être humain lui ouvre la voie.

A quoi lui servirait-il de gagner l'univers, sa puissance et toute sa richesse, s'il venait à perdre son âme? La question se renouvelle aujourd'hui devant la force de l'éthique de la prospérité. Ce qui est en jeu, ce n'est pas le bonheur des hommes dans l'accroissement de leurs richesses, c'est leur capacité d'exister en tant qu'hommes. A quoi leur servirait-il de trouver la fortune s'ils cessaient d'être ce qu'ils sont? C'est donc l'humanité qui est en jeu en chacun de nous.

La poursuite de la justice, favorisant le partage et créant une communauté de destin des personnes, qui contribuerait à l'actualisation d'une société idéale dans laquelle chacun se comporterait de telle façon que le bonheur de tous en serait accru, ne supprime nullement «l'obligation d'inventer" dans laquelle nous découvrons le sens de notre responsabilité. Même le bonheur ne peut nous dispenser de l'obligation d'être libres et responsables. Que ce bonheur se trouve dans la richesse et le bien-être matériel satisfaisant nos besoins et nos désirs dans cette vie, ou qu'il nous soit promis dans l'audelà, dans la contemplation éternelle de l'Etre suprême, ultime objet de nos désirs d'absolu, il n'en demeure pas moins que l'homme ne trouve sa valeur propre et le sens de son existence que dans l'invention morale que lui inspire l'obligation. Que l'hypothèse de Dieu s'impose à l' intelligence ou que cette dernière soit capable de s'en passer ne change rien à la condition de l'existence qui oblige l'être humain à se dépasser pour être. La véritable immanence du bonheur se trouve dans un être qui est déjà au-delà de luimême. Il n'y a donc pas d'autre issue que de faire croître l'humain dans chacun de nos actes. La croissance de l'humain dans le monde et dans l'histoire est donc un enjeu dans lequel ma responsabilité est engagée. La réponse dernière qu'il nous sera possible de donner aux questions que soulève l'éthique de la prospérité dans un monde où l'économie domine, est de savoir si l'homme gagne ou perd, et ce que chacun doit faire pour qu'il gagne. Tout le reste n'est que littérature.

Si nous sommes libres de tout, y compris, à la limite de Dieu lui-même, nous sommes individuellement responsables de tout. C'est la seule certitude qui puisse diriger nos actes. 


\section{Bibliographie}

ALBEDA, Wil (1984) De crisis van de werkgelegenheid en de verzorgingsstaat, Kampen, J.-H. Kok

ALBERT, Michel (1991) Capitalisme contre capitalisme, Paris, Editions du Seuil ARISTOTE, Métaphysique, trad. de J. Tricot (1933), Paris, Vrin

ARISTOTE, Ethique de Nicomaque, trad. de Jean Voilquin (1950), Paris, Garnier ARON, Raymond (1948) (édit. augmentée 1981) Introduction à la philosophie de l'histoire, Essai sur les limites de l'objectivité historique, Paris, Gallimard

ARONDEL, Philippe (1991) Morale sociale chrétienne et discours libéral, Paris, Editions Mame

BARNI, Jules (1992) (1868) La morale dans la démocratie, Préface de Pierre Macherey, Paris, Kimé

BASTIDE, George (1939) Le moment historique de Socrate, Paris, Alcan

BASTIDE, George (1943) Les grands thèmes moraux de la civilisation occidentale, Grenoble, Bordas

BASTIDE, George (1953) Méditations pour une éthique de la personne, Paris, PUF

BASTIDE, George (1961) Traité de l'action morale, Paris PUF

BEAUD, Michel (1990) (4e édit.) Histoire du capitalisme, Paris, Seuil

BERGSON, Henri (1946) (48e édit.) Les deux sources de la morale et de la religion, Paris, PUF

BOLLE DE BAL, Marcel (1985) La tentation communautaire, Les paradoxes de la reliance et de la contre-culture, Bruxelles, Editions de l'Université de Bruxelles

BOLLE DE BAL, Marcel (1989) Les doubles jeux de la participation, Rémunération, performance et culiure, Bruxelles, Presses Interuniversitaires européennes

BOULDING, Kenneth E. (1953) The Organizational Revolution, A Study in the Ethics of Economic Organizations, New York, Harper and Brothers

BRESSON, Yoland et GUILHAUME, Philippe (1986) Le participat, Réconcilier l'économique et le social, Paris, Chotard et assoc.

BRUN, Jean (1959) L'épicurisme, Paris, PUF, coll. Que sais-je

BRUN, Jean (1963) (2e édit.) Socrate, Paris, PUF, coll. Que sais-je

BRUNSCHVICG, Léon (1953) (2e édit.) Le progrès de la conscience dans la philosophie occidentale, Paris, PUF 
CHAUCHARD, Paul (1959) Biologie et morale, Tours, Mame

CHAUNU, Pierre (1987) La liberté, Paris, Fayard

CLAIR, André (1989) Ethique et humanisme, Essai sur la modernité, Paris, Cerf CONFUCIUS, Pensées morales (1953) Traduit du Chinois par René Brémond, Paris, Plon

COTTA, Alain (1991) Le capitalisme dans tous ses états, Paris, Fayard

de BEAUVOIR, Simone (1962) Pour une morale de l'ambiguĭté (nouvelle édition) Paris, Gallimard, coll. Idées

D'AVIAU DE TERNAY, Henri (1992) La liberté Kantienne, un impératif d'exode, Préface de Guy Petitdemange, Paris, Editions du Cerf

DELBOS, Victor (1905) La philosophie pratique de Kant, Paris, Alcan

DOMENACH, Jean-Marie (1992) Une morale sans moralisme, Paris, Flammarion

DUPREEL, Eugène (1932) Traité de morale, Bruxelles, Edition de la Revue de l'Université de Bruxelles

DUPREEL, Eugène (1939) Esquisse d'une philosophie des valeurs, Paris, Alcan DUPUY, Maurice (1959) La philosophie de Max Scheler, Son évolution et son unité, 2 Vol., Paris, PUF

DURKHEIM, Emile (1911) «Jugements de valeur et jugements de réalité», Atti del IV Congresso Internationale di Filosofia, Genova, Formiggini, (s.d.)

DURKHEIM, Emile (1963) (1924) Sociologie et philosophie, Paris, PUF

ETCHEGOYEN, Alain (1991) La valse des éthiques, Paris, Editions François Bourin

EWALD, François, (1986) L'Etat providence, Paris, Grasset

FLETCHER, Joseph (1966) Situation Ethics, The New Morality, Philadelphia, The Westminster Press

FLETCHER, Joseph (1967) Moral Responsibility, Situation Ethics at Work, Philadelphia, The Westminster Press

FLUGEL, J. C. (1962) Man, Morals and Society, London, Penguin Books

FOURASTIE, Jean (1979) Les trente glorieuses ou la révolution invisible de 1946 d 1975, Paris, Fayard

FRIEDMAN, David (1992) (1973-78) Vers une société sans Etat, trad. par Françoise Liégeois, Paris, Les Belles Lettres

FULLIQUET, George (1898) Essai sur l'obligation morale, Paris, Alcan

GALBRAITH, John Kenneth (1958) The Affluent Society, London, Hamish Hamilton, Penguin Books 
GENARD, Jean-Louis (1992) Sociologie de l'éthique, Paris, L'Harmattan, Préface de Claude Javeau

GILSON, Etienne (1944) (2e édit.) L'esprit de la philosophie médiévale, Paris, Vrin GINSBERG, Morris (1962) (1956) On the Diversity of Morals, London, Mercury Books

GORZ, André (1977) Fondements pour une morale, Paris, Galilée

GORZ, André (1988) Métamorphoses du travail, Quête du sens, Critique de la raison économique, Paris, Galilée

GURVITCH, Georges (1961) (3e édit.) Morale théorique et science des moeurs, Paris, PUF

GUYAU, Jean-Marie (1921) (16e édit.) (1885) Esquisse d'une morale sans obligation ni sanction, Paris, Alcan

HABERMAS, Jürgen (1978) (1973) Raison et légitimité, Problèmes de légitimation dans le capitalisme avancé, Trad. de Jean Lacoste, Paris, Payot

HEIDEGGER, Martin (1958) Introduction à la métaphysique, trad. par Gilbert Kahn, Paris, PUF

HERSCH, Jeanne (1964) (nouv, éd.) L'illusion philosophique, Paris, Plon

HÖFFE, Otfried (1991) La justice politique, Fondement d'une philosophie critique du droit et de l'Etat, trad. par Jean-Christophe Merle, Paris, PUF

HUDE, Henri (1992) Ethique et politique, Paris, Editions universitaires et Mame ISTAS, Michel (1986) Les morales selon Max Weber, Paris, Cerf

JALLADE, Jean-Pierre, Ed. (1988) The Crisis of Redistribution in European Welfare States, Stoke-on-Trent, Trentham Books

JANKELEVITCH, Vladimir (198L) Le paradoxe de la morale, Paris, Seuil

JEANSON, Francis (1965) Le problème moral et la pensée de Sartre, Nouvelle édition, Paris, Seuil

JONAS, Hans (1991) Le Principe responsabilité, Une éthique pour la civilisation technologique, (Traduit de l'allemand par Jean Greisch) Paris, Les éditions du Cerf - Das Prinzip Verantwortung (1979) Frankfurt a. M., Insel Verlag

JUNG, C. G. (1988) (1961) Présent et avenir, Trad. de Roland Cahen, Paris, Buchet/ Chastel

KANT, Emmanuel (1960) (1788) Critique de la raison pratique, Trad. de François Picavet, Paris, PUF

KANT, Emmanuel (1964) (1785) Fondements de la métaphysique des moeurs, Trad, de Victor Delbos, Paris, Delagrave

KIMMAN, E.J.J.M. (1990) Marktethiek, Assen, Van Gorcum 
KRÜGER, Gerhard (1961) Critique et morale chez Kant, trad. par M. Regnier, Paris, Beauchesne - Philosophie und Moral in der Kantischen Kritik

LALANDE, André (1988) (16e édit.) (1923) Vocabulaire technique et critique de la philosophie, Société française de philosoophie, Paris, PUF

LADRIERE, Paul et GRUSON, Claude (1992) Ethique et gouvernabilité, «Un projet européen», Paris, PUF

LAVELLE, Louis (1951 - 1955) Traité des valeurs, 2 Vol. Paris, PUF

LEPP, Ignace (1963) La morale nouvelle, Paris, Grasset

LE SENNE, René (1949) Traité de morale générale, Paris, PUF

LE SENNE, René (1950, 2e édit.) Le devoir, Paris, PUF

LEVINAS, Emmanuel (1982) Ethique et infini, Dialogues avec Philippe Nemo, Paris, Fayard - Le livre de poche

LEVY-BRUHL, Lucien (1922) La mentalité primitive, Paris, Alcan

LEVY-BRUHL, Lucien (1953) (15e édit.) (1903) La morale et la science des moeurs, Paris, PUF

LIPOVETSKY, Gilles (1992) Le crépuscule du devoir, L'éthique indolore des nouveaux temps démocratiques, Paris, Gallimard

MacINTYRE, Alasdair (1985) (Secd. edit.) (1981) After Virtue, A study in moral theory, London, Duckworth

MADINIER, Gabriel (1963) La conscience morale, Paris, PUF

MARCEL, Gabriel (1935) Etre et avoir, Paris, Montaigne

MARITAIN, Jacques (1960) La philosophie morale, Paris, Gallimard

MARITAIN, Jacques (1990) (1951) Neuf leçons sur les notions premières de la philosophie morale, Oeuvres complètes vol. IX, Fribourg, Editions universitaires, Paris, Saint-Paul

MEHL, Roger (1957) De l'autorité des valeurs, Paris, PUF

MEHL, Roger (1971) Les attitudes morales, Paris, PUF

MOORE, George Edward (1954) (1903) Principia Ethica, Cambridge, The University Press

MOREAU, Marcel (1979) Discours contre les entraves, Paris, Christian Bourgeois

NABERT, Jean (1943) Eléments pour une éthique, Paris, PUF

NANCY, Jean-Luc (1983) L'impératif catégorique, Paris, Flammarion

NIETZSCHE, Friedrich (1980) (1884) Also Sprach Zarathustra, Werke in Vier Bände, Vol. I, Wien, Caesar Verlag 
NIETZSCHE, Friedrich (1954) (1886) Par delà le Bien et le Mal, trad. de Henri Albert, Paris, Mercure de France

OLLE-LAPRUNE, Léon (1989) (1880) De la certitude morale, Paris, Editions universitaires

PATTERSON, Orlando (1991) Freedom, Vol. I, Freedom in the Making of Western Culture, Basic Books, USA

PERELMAN, Chaïm (1980) Introduction historique à la philosohie morale, Bruxelles, Editions de l'Université de Bruxelles

PLATON, Oeuvres complètes, (1963) trad. de Léon Robin, Paris, Gallimard, coll. La Pléiade

POLIN, Raymond (1968) Ethique et politique, Paris, Editions Sirey

POPPER, Karl R. (1991) (1945) The Open Society and Its Enemies, (2 Vol.) London, Routledge

PRICHARD, H. A. (1957) (1949) Moral Obligation, Essays and Lectures, Oxford, Clarendon Press

RAWLS, John (1987) (1971) Théorie de la Justice, trad. par Catherine Audard, Paris, Seuil

REICH, Robert B. (1991) The Work of Nations, New York, Alfred E. Knopf

ROBIN, Léon (1963) (3e édit.) La morale antique, Paris, PUF

ROSANVALLON, Pierre (1981) La crise de l'Etat-providence, Paris, Editions du Seuil

SARTRE, Jean-Paul (1946) L'existentialisme est un humanisme, Paris, Les Editions Nagel

SARTRE, Jean-Paul (1943) L'être et le néant, Paris, Gallimard

SARTRE, Jean-Paul (1951) Le Diable et de Bon Dieu, Paris Gallimard

SAUVAGE, Micheline (1956) Socrate et la conscience de l'homme, Paris, Seuil, coll. Maîtres spirituels

SCHELER, Max (1955) (1926) Le formalisme en éthique et l'éthique matériale des valeurs, Traduct. de Maurice de Gandillac, Paris, Gallimard

SCHOOYANS, Michel (1991) La dérive totalitaire du libéralisme, Paris, Editions universitaires

SESONSKE, Alexander (1964) (1957) Value and Obligation, The Foundations of an Empiricist Ethical Theory, New York Galaxy Book

SIMMEL, Georg (1987) Philosophie de l'argent, Paris, PUF; traduit de Philosophie des Geldes (1900), Neue Deutsche Ausagabe, Berlin, Dunker \& Humblot (1977)

SORMAN, Guy (1984) La solution libérale, Paris, Fayard 
SORMAN, Guy (1985) L'Etat minimum, Paris, Albin Michel

STOFFAËS, Christian (1987) Fins de mondes, Déclin et renouveau de l'économie, Paris, Odile Jacob

TOURAINE, Alain (1992) Critique de la modernité, Paris, Fayard

VAN PARIJS, Philippe (1991) Qu'est-ce qu'une société juste?, Paris, Seuil

VERGARA, Francisco (1992) Introduction aux fondements philosophiques du libéralisme, Paris, La Découverte

VIALATOUX, Joseph (1963) La morale de Kant, Paris, PUF

WEBER, Max (1964) (1905) L'éthique protestante et l'esprit du capitalisme, tad. de l'all., Paris, Plon

WILLIAMS, Bernard (1990) L'éthique et les limites de la philosophie, Paris, Gallimard; traduit de Ethics and the Limits of Philosophy (1985), London, William Collins Sons \& Co.

WOLFF, Christian, (1972 ff.) (1713-1754) Complete Works, Olms Editions, Hildensheim-New York 


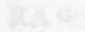

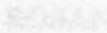

nas

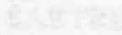

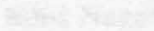

$9 x^{2}=17$

$5 x^{2}$

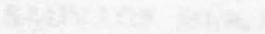

$x^{2}+\frac{1}{2}$

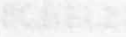

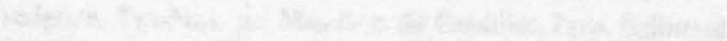

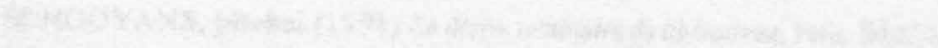

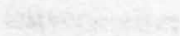

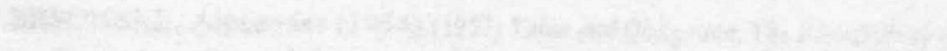
20.

ant

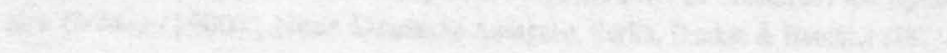

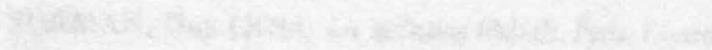




\title{
De morele verplichting en de ethiek van de welvaart
}

\author{
Terug naar de verantwoordelijke persoon
}

\section{SAMENVATTING}

Hoofdstuk 1 Dit proefschrift bestaat uit een filosofisch onderzoek van het begrip morele verplichting. Deze studie streeft ernaar een nieuw perspectief te bieden als praktisch antwoord op de ethische vragen die opgeroepen worden door de recente ontwikkelingen van de economische en sociale stelsels. Het uitgangspunt is dat de economie van vandaag, voortdurend meer en meer materiële goederen kan en wil produceren, eist dat de individuele mens totaal ter beschikking van de bevordering van deze economische aktiviteiten staat. De mens is gevangen in een waardensysteem dat op produktie gericht is. De mens wordt alleen maar bekeken als een dienaar van het stelsel, met als enige opgave het systeem zelf te laten functioneren, als producent of als consument van deze goederen. Het individu heeft het statuut van verantwoordelijke persoon verloren, omdat de «ethiek van de welvaart» geen andere rechtvaardiging vindt dan de accumulatie van materiële rijkdom. Prestatie en succes worden de ultieme waarden van zo'n menselijke bestaan.

Hoofdstuk 2 Het idee van morele verplichting, of kortweg de plicht, wordt historisch in drie stappen in de westerse filosofie ontwikkeld. Eerst door de ontwikkeling van het begrip "zelf-bewust zijn» in verband met het griekse "ken jezelf» (Sokrates), dan door de onthulling van de intentionaliteit van de menselijke aktie in haar relatie tot het absolute (christendom), tenslotte door de bevestiging van de autonomie van het verstand, waardoor de verplichting de formele basis van de ethiek wordt (Kant). Daarna wordt uiteengezet hoe het bewustzijn van deze verplichting een onmiddellijk gegeven van het subjektieve geweten wordt, hoe het dus onmogelijk is geworden om daarna een objektieve en wetenschappelijke verklaring van 
de verplichting te geven zonder haar als zodanig te vernietigen. Dit is geillustreerd door vier kritische analysen: eerst een psychologische uitleg (Fulliquet), daarna een vitalistische(Guyau), dan een sociologische(Durkheim), en eindelijk een veelomvattende sociaal-vitalistische uiteenzetting (Bergson). Als konklusie wordt gesteld dat de taak van de huidige filosofie erin bestaat een nieuwe theorie van de morele verplichting te formuleren om de existentiële ethische vragen van onze tijd te kunnen beantwoorden.

Hoofdstuk 3 Deze nieuwe theorie is gebouwd op de twee dimensies van de ethiek: de subjektieve, formele dimensie waardoor de subjektiviteit van de morele mens naar voren komt; en een objektieve, materiële dimensie, gebaseerd op een normatieve relatie met anderen, zodat ze intersubjektieve objektiviteit kan worden genoemd. Men erkent dat er een moreel feit bestaat in die zin dat de mens als de enige auteur van zijn daden beschouwd moet worden. Een niet-subjektieve morele beoordeling is zodoende mogelijk waardoor de menselijke daden als goed of slecht beoordeeld kunnen worden. Dit ethisch «bewust worden» is de oorsprong van een nieuwe kennis van de mens zelf, die in dit boek morele anthropologie wordt genoemd.

Hoofdstuk $4 \quad$ Uitgaande van deze erkenning van de twee dimensies van de ethiek wordt de konkreet beleefde ervaring van de morele verplichting geanalyseerd. Dit helpt de verschillende betekenissen van de subjektiviteit zelf te verhelderen en haar relatie tegenover de anderen. De beleefde ervaring van de morele verplichting en haar veeleisendheid is de oorsprong van een menselijke «zelf-erkenning» en draagt dus direkt bij aan de opbouw van het subjekt tot complete menselijke persoon. Morele filosofie wordt dus een originele «filosofie van het bestaan», een soort metaphysika van de menselijke aktie, als een voorwaarde voor het «mens worden». De morele aktie wordt aldus gezien als een gelegenheid om zichzelf tot persoon te ontwikkelen.

Hoofdstuk $5 \quad$ Omdat de sociale dimensie van ethiek de mens in zijn relatie tot anderen stelt, staat het idee van samenleving centraal in de analyse van de morele verplichting. In dit verband word thet verschil tussen verplichting en de verschillende vormen van morele dwang beschreven. Dit kan diffuus en onbewust, of openbaar en wettelijk georganiseerd zijn, en drukt vaak heel zwaar op de menselijke handelingen. Deze analyse onderstreept hoe gevaarlijk het voor de moraliteit kan zijn, indien zij tot een aanpassing van gedrag aan uiterlijke eisen gereduceerd wordt.

Hoofdstuk 6 De sociale dwang moet inderdaad overstegen worden. Dat betekent dat de samenleving niet alleen het gesloten milieu voor een beperkende ethiek moet zijn, maar ook de kans moet geven tot de creatie van nieuwe waarden. In dit hoofdstuk worden diverse morele attitudes geanalyseerd en wordt angetoond hoe de morele verplichting verder gaat in de opbouw van de moraliteit dan de traditionele deugd-ethiek in de richting van een maatschappelijke verplichting ten opzichte van de anderen. 
Hoofdstuk 7 De «ethiek van de welvaart» wordt beschreven als de dominante ethiek in onze tijd, met kenmerken zoals de ideologie van de welvaart en een overheersend individualistisch kapitalisme. Deze ethiek wordt gezien als een echte sociale dwang die, omdat zij exclusief op kwantiteit is gebouwd, in het geheel geen bevredigende doelstelling voor de mens kan betekenen. Een aanvaardbare sociale ethiek tegen het neoliberalisme in de huidige economie gaat uit van een verantwoordelijk subjekt dat zich bewust is van zijn verplichting ten opzichte van hemzelf en van de anderen.

Hoofdstuk 8 De nieuwe ethiek wordt beschreven als een rechtvaardigheidsethiek. Het kan niet een abstrakte en tijdloos met perfekte begrippen beschreven rechtvaardigheid zijn, maar alleen een historische, dynamische realiteit die door individuen en maatschappij bevorderd kan worden. Deze ethiek van de recthvaardigheid moet de notie van de oude welvaartsethiek vervangen. De staten en sociale organisaties zouden dit als een fundamentele verplichting moeten beschouwen. Rechtvaardigheid betekent meer dan inkomensherverdeling, een soort gemoraliseerde «verzorgingsstaat», maar veeleer als een gezamenlijke participatie in welvaart, welzijn en verantwoordelijkheid. Dus het subjekt wordt niet aan een oppervlakkig egalitarisme onderworpen, maar is lid van een bewuste solidariteit.

Hoofdstuk 9 Tenslotte, probeert het boek een uiteenzetting te geven over de betekenis van de evolutie van de moraliteit in de geschiedenis. Hier wordt onderstreept dat, al is het mogelijk over een vooruitgang van morele waarden door de eeuwen te spreken, dit niet geldt voor de beleefde moraliteit. Ideeën hebben is niet voldoende; de moraliteit moet in ieder individueel leven opnieuw beleefd worden door daden geïnspireerd door morele verplichting. De mens is verantwoordelijk voor zijn eigen vooruitgang. 


\section{CURRICULUM VITAE}

Gabriel Fragnière est né à Lausanne (Suisse) le 4 mars 1934. Il termine ses études secondaires à la Stiftschule, Engelberg, et poursuit des études de philosophie et lettres à l'Université de Lausanne, où il obtient le titre de Licencié ès Lettres avec un mémoire sur «La philosophie de la personne chez Jacques Maritain». Il poursuit des études de science politique et de sociologie au Collège d'Europe de Bruges, et complète sa formation par deux années de recherches (1966-1968) aux Etats-Unis, Université de Stanford et American University (Washington D.C.).

Depuis 1969, il poursuit une carrière de recherches en matière d'éducation et de formation au niveau européen, et participe activement à la mise en place de structures de coopération universitaire en Europe. Il a été, de 1980 à 1981, Directeur du Centre européen travail et société, à Maastricht, et est actuellement Directeur du département d'études européennes à la «Central European University» à Prague.

Parmi ses publications principales il faut noter:

Le royaume de l'homme, Essai sur la religion et la démocratie (Genève, Les éditions du Mont-Blanc, 1973)

L'éducation créatrice, Fondation européenne de la culture (Paris-Bruxelles, Elsevier Sequoia, 1975)

L'homme et la vie, Biologie contemporaine et éthique (Paris, Le Centurion, 1978)

Le non-travail (Werken zonder Banen) (Bruxelles, Fondation Roi Baudoin, 1985)

Formation et condition humaine au XXIe siècle (Bruxelles, Presses Interuniversitaires Européennes, 1991) 
